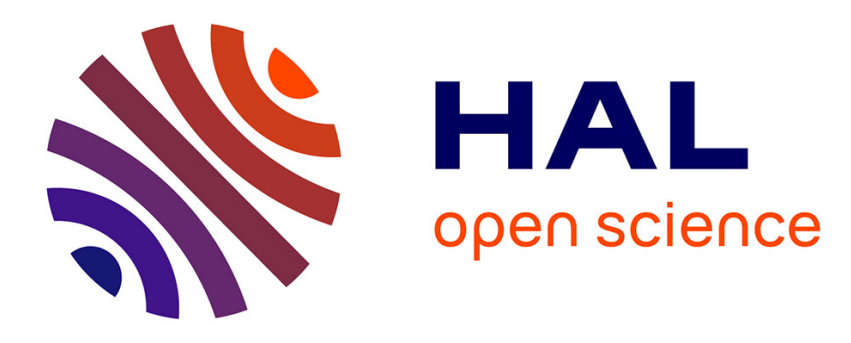

\title{
Refined probabilistic global well-posedness for the weakly dispersive NLS
}

Chenmin Sun, Nikolay Tzvetkov

\section{To cite this version:}

Chenmin Sun, Nikolay Tzvetkov. Refined probabilistic global well-posedness for the weakly dispersive NLS. 2020. hal-02977481

\section{HAL Id: hal-02977481 \\ https://hal.science/hal-02977481}

Preprint submitted on 25 Oct 2020

HAL is a multi-disciplinary open access archive for the deposit and dissemination of scientific research documents, whether they are published or not. The documents may come from teaching and research institutions in France or abroad, or from public or private research centers.
L'archive ouverte pluridisciplinaire HAL, est destinée au dépôt et à la diffusion de documents scientifiques de niveau recherche, publiés ou non, émanant des établissements d'enseignement et de recherche français ou étrangers, des laboratoires publics ou privés. 


\title{
REFINED PROBABILISTIC GLOBAL WELL-POSEDNESS FOR THE WEAKLY DISPERSIVE NLS
}

\author{
CHENMIN SUN, NIKOLAY TZVETKOV
}

\begin{abstract}
We continue our study of the cubic fractional NLS with very weak dispersion $\alpha>1$ and data distributed according to the Gibbs measure. We construct the natural strong solutions for $\alpha>\alpha_{0}=\frac{31-\sqrt{233}}{14} \approx 1.124$ which is strictly smaller than $\frac{8}{7}$, the threshold beyond which the first nontrivial Picard iteration has no longer the Sobolev regularity needed for the deterministic well-posedness theory. This also improves our previous result in Sun-Tzvetkov 28. We rely on recent ideas of Bringmann 8 and DengNahmod-Yue [17]. In particular we adapt to our situation the new resolution ansatz in 17. which captures the most singular frequency interaction parts in the $X^{s, b}$ type space. To overcome the difficulties caused by the weakly dispersive effect, our specific strategy is to benefit from the "almost" transport effect of these singular parts and to exploit their $L^{\infty}$ as well as the Fourier-Lebesgue property in order to inherit the random feature from the linear evolution of high frequency portions.
\end{abstract}

\section{CONTEnTs}

1. Introduction 2

1.1. Motivation 2

1.2. Setup and the main result 2

1.3. Boundedeness of the Picard iterates in $L^{\infty} \quad 4$

1.4. Difficulties and the Strategy 5

\begin{tabular}{ll}
\hline 1.5. & Refined resolution ansatz \\
\hline
\end{tabular}

Acknowledgment 9

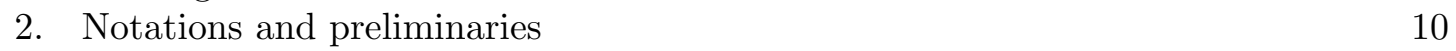

$\begin{array}{ll}2.1 . & \text { General notations } \\ & 10\end{array}$

$\begin{array}{lll}2.2 . & \text { Spaces for functions and operators } & 10\end{array}$

$\begin{array}{lll}2.3 . & \text { Counting lemmas and the Strichartz inequality } & 12\end{array}$

\begin{tabular}{lll}
\hline 2.4. & Estimates for operators & 13
\end{tabular}

\begin{tabular}{lll}
\hline 2.5. Probability tool-box & 15
\end{tabular}

\begin{tabular}{lll}
\hline 3. & Key iterative steps & 17
\end{tabular}

\begin{tabular}{ll}
3.1. & Rigorous resolution scheme \\
\hline 3.2. & 17
\end{tabular}

$\begin{array}{lll}\text { 3.2. Key multi-linear terms } & 18\end{array}$

$\begin{array}{lr}3.3 . & 19\end{array}$

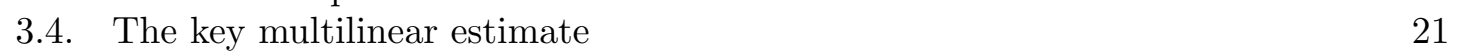

3.5. Proof of the main theorem 23

3.6. Sketch of the convergence of the whole sequence 25

4. $L^{\infty}$ and Fourier-Lebesgue property for paracontrolled objects 26

5. Mapping properties of the operator $\mathcal{P}_{N, L}^{+} \quad 28$

5.1. $S^{b, q}$-mapping properties of the operator $\mathcal{P}_{N, L}^{+} \quad 29$

$\begin{array}{lll}5.2 . & X^{0,6} \text {-mapping property of the operator norm of } \mathcal{P}_{L}^{ \pm} & 32\end{array}$

\begin{tabular}{ll|l}
6. Low modulation reduction & 36
\end{tabular}

6.1. $\quad$ Modulation reduction for the estimates of operator kernels 36

$\begin{array}{lll}6.2 . & \text { Modulation reduction for the trilinear estimates } & 39\end{array}$

$\begin{array}{ll}\text { 7. } & \text { Multilinear estimate for the kernel } \mathcal{P}_{N, L}^{+}\end{array}$

$\begin{array}{lll}\text { 7.1. Notational simplifications } & 41\end{array}$

$\begin{array}{lll}7.2 . & \text { Algorithms and reductions } & 42\end{array}$

$\begin{array}{lll}7.3 . & \text { Implementing the algorithms } & 47\end{array}$ 


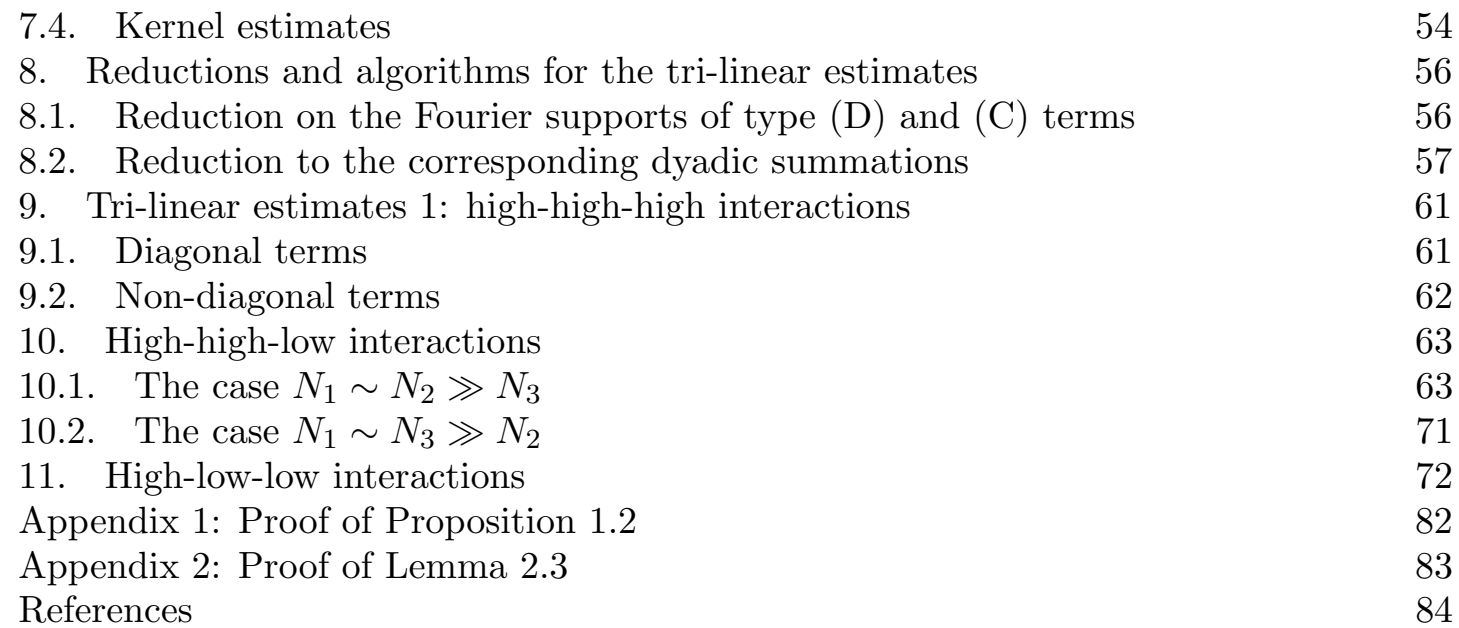

\section{INTRODUCTION}

1.1. Motivation. In this article, we continue our study of the defocusing cubic fractional nonlinear Schrödinger equation (FNLS)

$$
i \partial_{t} u+\left|D_{x}\right|^{\alpha} u+|u|^{2} u=0, \quad(t, x) \in \mathbb{R} \times \mathbb{T},
$$

where $u$ is complex-valued and $\left|D_{x}\right|^{\alpha}=\left(-\partial_{x}^{2}\right)^{\alpha / 2}$ is defined as the Fourier-multiplier $\left.\left|\widehat{\left.D_{x}\right|^{\alpha}} f(k)=\right| k\right|^{\alpha} \widehat{f}(k)$. The parameter $\alpha$ measures the strength of the dispersion. In this article, we are always in the weak dispersive regime where $1<\alpha<2$. The equation (1.1) is a Hamiltonian system with conserved energy functional

$$
H(u)=\left.\left.\int_{\mathbb{T}}|| D_{x}\right|^{\frac{\alpha}{2}} u\right|^{2} d x+\frac{1}{2} \int_{\mathbb{T}}|u|^{4} d x .
$$

Moreover, the mass $M(u)=\int_{\mathbb{T}}|u|^{2} d x$ is also conserved along the flow of (1.1). The fractional Schrödinger equation was introduced in the theory of the fractional quantum mechanics where the Feynmann path integrals approach is generalized to $\alpha$-stable Lévy process [24]. Also, it appears in the water wave models (see [19] and references therein). In addition, we refer to [23] where the fractional NLS on the line appears as a limit of the discrete NLS with long range interactions.

The motivation in our previous work [28] is to provide macroscopic properties for the solutions of (1.1), and in particular to detect the strength of the dispersion in the construction of the Gibbs measure. In that work, we construct global solutions on a full measure set with respect to the Gibbs measure by different methods, depending on the value of $\alpha$. More precisely, when $\alpha>\frac{6}{5}$, we construct the global strong solution satisfying the recurrence properties and show that the sequence of smooth solutions for FNLS with truncated initial data converges almost surely to the constructed strong solution. When $1<\alpha \leq \frac{6}{5}$, we rely on a simple method of Bourgain-Bulut [5, 6, 7] to prove the convergence of the Galerkine approximation scheme for the FNLS with truncated both data and nonlinearity. However, we were not able to show that the limit constructed by that method satisfies the flow property and therefore it is a natural question to investigate whether there exists global strong solution in the full range $\alpha>1$ on the support of the Gibbs measure and if the strong solution coincides with the limit constructed by the Galerkine approximation scheme.

1.2. Setup and the main result. To present the main result and to explain the different methods of constructing solutions, we recall the standard randomization procedure. Let $\left(g_{k}\right)_{k \in \mathbb{Z}}$ be a sequence of independent, standard complex-valued Gaussian random variables 
on a fixed probability space $(\Omega, \mathcal{F}, \mathbb{P})$. Denote by $\mu$ the Gaussian measure on $H^{\frac{\alpha-1}{2}-\epsilon}(\mathbb{T})$ for any $\epsilon>0$ induced by the map

$$
\omega \longmapsto \phi^{\omega}(x):=\sum_{k \in \mathbb{Z}} \frac{g_{k}(\omega)}{[k]^{\frac{\alpha}{2}}} \mathrm{e}_{k}(x),
$$

where $\mathrm{e}_{k}(x)=\mathrm{e}^{i k x}$ and $[k]^{\frac{\alpha}{2}}=\left(1+|k|^{\alpha}\right)^{\frac{1}{2}}$. Set $E_{n}=\operatorname{span}\left\{\mathrm{e}_{k}:|k| \leq n\right\}$. We denote by

$$
\Pi_{n}: H^{\frac{\alpha-1}{2}-\epsilon}(\mathbb{T}) \longrightarrow E_{n}
$$

the corresponding spectral projection. When $\alpha>1$, it is well-known that for any $0 \leq$ $\sigma_{0}<\frac{\alpha-1}{2},\left\||D|^{\sigma_{0}} u\right\|_{L^{\infty}(\mathbb{T})}$ is $\mu$-almost surely finite. Then the Gibbs measure $\rho$ associated with $(1.1)$ is

$$
d \rho(u)=\mathrm{e}^{-\frac{1}{2} \int|u|^{4}} d \mu(u) .
$$

This measure can be viewed as the limit of $\rho_{n}$, the Gibbs measure associated with the truncated Hamiltonian

$$
H_{n}(u)=\left.\left.\int_{\mathbb{T}}|| D_{x}\right|^{\frac{\alpha}{2}} \Pi_{n} u\right|^{2} d x+\frac{1}{2} \int_{\mathbb{T}}\left|\Pi_{n} u\right|^{4} d x
$$

whose associated Hamiltonian flow is the truncated FNLS (ODE):

$$
i \partial_{t} v_{n}+\left|D_{x}\right|^{\alpha} v_{n}+\Pi_{n}\left(\left|\Pi_{n} v_{n}\right|^{2}\right)=0,\left.\quad v_{n}\right|_{t=0}=\Pi_{n} \phi .
$$

Once the Gibbs measure $\rho$ is constructed, we need to construct the dynamics on the support of the measure, namely to solve (1.1) with randomized initial data (1.2).

There are two ways to solve the dynamical problem, the first is to prove the convergence of (1.3), since for each fixed $n$, the truncated FNLS admits a global solution, as it is a Hamiltonian ODE on the finite dimensional space $E_{n}$. In [28], using the Bourgain-Bulut argument, we have proved:

Theorem 1 ([28]). Assume that $\alpha>1$ and $\sigma_{0}<\frac{\alpha-1}{2}$. The sequence $\left(v_{n}^{\omega}\right)_{n \in \mathbb{N}}$ of solutions of $(1.3)$ with randomized initial data $(1.2)$ converges a.s. in $C\left(\mathbb{R} ; H^{\sigma_{0}}(\mathbb{T})\right)$ to some limit $v$ which solves (1.1) in the distributional sense.

The second approximation, more natural from the PDE view-point, is to consider the convergence of the sequence of smooth solutions $u_{n}$ of

$$
i \partial_{t} u_{n}+\left|D_{x}\right|^{\alpha} u_{n}+\left|u_{n}\right|^{2} u_{n}=0,\left.\quad u_{n}\right|_{t=0}=\Pi_{n} \phi .
$$

Note that for each fixed $n$, the global well-posedness of $(1.4)$ is guaranteed, thanks to a theorem proved (in the range $\alpha>\frac{2}{3}$ ) in [29] or [13] (in the range $\alpha>1$ ). The major difference of the aforementioned approximations is that for the PDE approximation, we need to establish a probabilistic local well-posedness which provides us more information on the structure of the solution. While only to prove the convergence for the first approximation, some probabilistic compactness methods exploiting the invariance of the finite dimensional Gibbs measure $\rho_{n}$ are sufficient, see for example [5], [6], [7], [10, [26] in the context of nonlinear Schrödinger and nonlinear wave equations. Therefore, a natural question can be formulated as follows:

Question 1.1. Can we show that for $\alpha>1$, the sequence $\left(u_{n}^{\omega}\right)_{n \in \mathbb{N}}$ of solutions of (1.4) with randomized initial data $\left(1.2\right.$ converges a.s. in $C\left(\mathbb{R} ; H^{\sigma_{0}}(\mathbb{T})\right)$ to some unique limit $u$ which coincides with the limit obtained in Theorem 11? Moreover, can we define the solution map $\Phi(t)$ satisfying the flow property and Poincaré's recurrence property on a full measure set with respect to the Gibbs measure?

We will call strong solutions those obtained when giving a positive answer of Question 1.1. The threshold $\alpha>1$ is designed for two reasons. Firstly, we do not need to renormalize the equation as the initial data lives in $L^{\infty}$ almost surely. Secondly, as we will see later, for $\alpha>1$, the second Picard's iteration enjoys some smoothing effect, due to the presence of the dispersion. 
The main result of this article is the following partial answer of Question 1.1 which improves our previous result in [28] for $\alpha>\frac{6}{5}$.

Theorem 2. Assume that $\alpha>\alpha_{0}=\frac{31-\sqrt{233}}{14}$ and $\sigma_{0}<\frac{\alpha-1}{2}$. Then the sequence of smooth solutions $\left(u_{n}^{\omega}\right)_{n \in \mathbb{N}}$ of

$$
i \partial_{t} u_{n}+\left|D_{x}\right|^{\alpha} u_{n}+\left|u_{n}\right|^{2} u_{n}=0,\left.\quad u_{n}\right|_{t=0}=\sum_{|k| \leq n} \frac{g_{k}(\omega)}{[k]^{\frac{\alpha}{2}}} \mathrm{e}_{k},
$$

converges almost surely in $C\left(\mathbb{R} ; H^{\sigma_{0}}(\mathbb{T})\right)$ to a limit which solves 1.1 .

Let us give a brief explanation about the number $\alpha_{0}$ appearing in the above statement. The important feature is that the number $\alpha_{0}$ appearing in Theorem 2 is smaller than $8 / 7$ which is the threshold beyond which the first nontrivial Picard iteration has no longer the Sobolev regularity needed for the deterministic well-posedness theory (see the discussion below for more details). For this reason we find that the progress made in this paper is at a conceptual level.

Following the argument in [28, we are able to show that the unique limit satisfies the flow property and the Gibbs measure $\rho$ is invariant under the flow. The key point is to establish a probabilistic local well-posedness result which provides a fine structure of the solution of (1.1). Let us mention that when $\alpha>\frac{4}{3}$, the above theorem is proved in 15$]$ using only the deterministic theory without appealing to any random oscillation effect. In [28], when $\frac{6}{5}<\alpha \leq \frac{4}{3}$, we go beyond the available deterministic theory by adapting the Da Prato-Debussche affine decomposition in conjugation with a gauge transformation to prove the probabilistic local well-posedness.

1.3. Boundedeness of the Picard iterates in $L^{\infty}$. To motive the necessity of a refined analysis and to compare with the context of parabolic equations, let us look at the formal Picard iteration scheme associated with our equation. Denote by

$$
z_{1}^{\omega}(t)=\mathrm{e}^{i t\left|D_{x}\right|^{\alpha}} \phi^{\omega},
$$

where $\phi^{\omega}$ is given by 1.2 . By formally expanding the solution of 1.1 as power series in terms of the initial data, we write

$$
Z^{\omega}(t)=\sum_{j=0}^{\infty} z_{2 j+1}^{\omega}(t)
$$

Formally inserting into the equation $\left(i \partial_{t}+\left|D_{x}\right|^{\alpha}\right) Z^{\omega}+\left|Z^{\omega}\right|^{2} Z^{\omega}=0$ and comparing the coefficients, $z_{2 j+1}^{\omega}$ should satisfy the equation:

$$
\left(i \partial_{t}+\left|D_{x}\right|^{\alpha}\right) z_{2 j+1}^{\omega}=-\sum_{\substack{j_{1}, j_{2}, j_{3} \geq 0 \\ j_{1}+j_{2}+j_{3}=j-1}} z_{2 k_{1}+1}^{\omega} \bar{z}_{2 j_{2}+1}^{\omega} z_{2 j_{3}+1},\left.\quad z_{2 j+1}^{\omega}\right|_{t=0}=0 .
$$

By induction we see that $z_{2 j+1}^{\omega}$ is a $(2 j+1)$-multilinear form of Gaussians:

$$
z_{2 j+1}^{\omega}(t, x)=\sum_{k_{1}, \cdots, k_{2 j+1}} c_{j}\left(t, k_{1}, \cdots, k_{2 j+1}\right) \frac{g_{k_{1}} \bar{g}_{k_{2}} \cdots \bar{g}_{k_{2 j}} g_{k_{2 j+1}}}{\left[k_{1}\right]^{\frac{\alpha}{2}} \cdots\left[k_{2 j+1}\right]^{\frac{\alpha}{2}}} \mathrm{e}_{k_{1}-k_{2}+\cdots-k_{2 j}+k_{2 j+1}}(x) .
$$

The following proposition shows that every finite order of Picard's iteration is bounded in $C\left([0, T] ; L^{\infty}(\mathbb{T})\right)$ :

Proposition 1.2. There exists $C_{0}>0$, such that for any $j \in \mathbb{N}, t \in \mathbb{R}, x \in \mathbb{T}$, we have

$$
\mathbb{E}\left[\left|z_{2 j+1}^{\omega}(t, x)\right|^{2}\right] \leq C_{0} t^{2 j}(2 j+1) !\left(\frac{(2 j-1) ! !}{j !}\right)^{2} .
$$


In particular, for any $T>0$ sufficiently small, there exists $\Omega_{T} \subset \Omega$, with $\mathbb{P}\left[\Omega_{T}\right]=1$, such that for any $\omega \in \Omega_{T}$ and any $j$, the partial sum of the Picard iteration satisfies

$$
Z_{2 j+1}^{\omega}(t):=\sum_{j^{\prime}=0}^{j} z_{2 j^{\prime}+1}^{\omega}(t) \in C\left([0, T] ; L^{\infty}(\mathbb{T})\right) .
$$

Though the partial sum of the formal expansion $Z$ is bounded in $L^{\infty}$, this proposition does not tell anything about the convergence of the remainder in the formal expansion $Z(t)=\sum_{j=0} z_{2 j+1}^{\omega}(t)$. Much effort has to been addressed to in order to prove the convergence of the remainder.

Let us also mention a comparison with parabolic equations. For $\alpha>1$, a typical function with respect to $\mu$ is an $L^{\infty}$ function. As a consequence, if we were dealing with a similar problem for a parabolic PDE then thanks to the nice $L^{\infty}$ mapping properties of the heat flow, the analysis would become essentially trivial. On the other hand, since we are dealing with a dispersive PDE, the linear problem is only well-posed in $L^{2}$ in the scale of the $L^{p}$ spaces which makes that even at positive regularities, refined detereministic estimates and probabilistic considerations are essential in the analysis.

1.4. Difficulties and the Strategy. Let us consider two extreme situations $\alpha=1$ and $\alpha=2$. When $\alpha=2$, the equation $(1.1)$ is the classical cubic Schrödinger equation which has nice dispersive properties. In particular, the $L^{4}$ Strichartz estimate holds with no loss of spatial derivative. When $\alpha=1$, the equation (1.1) is the cubic half-wave equation. If we ignore the nonlocal issue and consider only the transport equation

$$
i \partial_{t} u+i \partial_{x} u=|u|^{2} u
$$

we can solve this equation simply in the space $L^{\infty}$. These facts indicate that in the intermediate case $1<\alpha<2$, we should balance the dispersive effect and the transport property of the solutions according to different regimes. However, when $\alpha$ is very close to 1 , there are two major difficulties. Unlike the classical Schrödinger case $\alpha=2$, the $L^{4}$ Strichartz estimate loses almost $\frac{1}{8}$ derivatives (due to the degeneracy of the resonant function). Moreover, the fractional dispersion $\left|D_{x}\right|^{\alpha}$ is non-local which prevents us to use directly the transport property like (1.6).

Our strategy is based on the following observations. Firstly, the most singular parts in $X^{s, b}$ space come from the high-low-low type frequency interactions. These parts satisfy morally the transport equation. Secondly, the loss of derivatives in the Strichartz inequality occurs in the high-high-high or high-high-low frequency interaction regimes. Hence we should place the most singular part in the space $L^{\infty}$ instead of $X^{s, b}$ in these regimes when estimating tri-linear expressions. To realize this strategy, we use the refined resolution ansatz introduced by Deng-Nahmod-Yue in [17]. Roughly speaking, it concerns refining the affine ansatz and decomposing the solution roughly as $e^{i t\left|D_{x}\right|^{\alpha}} \phi^{\omega}+\Psi+w$ with a "random averaging operator" term $\Psi$ which captures the most singular frequency interactions. Additionally in our situation, the term $\Psi$ can be further decomposed into different parts carrying relatively "good" $L^{\infty}$ property and relatively "good" $X^{s, b}$ and Fourier-Lebesgue property.

The threshold $\alpha>\frac{6}{5}$ for the affine decomposition structure. To be more precise, we breifly recall the decomposition due to Bourgain [4] and Da Prato-Debussche [14] used in our previous work [28]. By using the gauge transformation

$$
v(t, x)=u(t, x) \mathrm{e}^{\frac{i t}{\pi} \int_{\mathbb{T}}|u|^{2} d x},
$$

we transform the FNLS as

$$
i \partial_{t} v+\left|D_{x}\right|^{\alpha} v=\mathcal{N}(v),\left.\quad v\right|_{t=0}=\phi^{\omega},
$$

where the Wick-ordered nonlinearity is given by

$$
\mathcal{N}(v):=-\mathcal{N}_{3}(v, v, v)+\mathcal{N}_{0}(v, v, v),
$$


and the trilinear forms $\mathcal{N}_{3}(\cdot, \cdot, \cdot)$ and $\mathcal{N}_{0}(\cdot, \cdot, \cdot)$ are defined as

$$
\begin{aligned}
& \mathcal{N}_{3}\left(f_{1}, f_{2}, f_{3}\right):=\sum_{\substack{k_{1}, k_{2}, k_{3} \\
k_{2} \neq k_{1}, k_{3}}} \widehat{f_{1}}\left(k_{1}\right) \widehat{\widehat{f_{2}}}\left(k_{2}\right) \widehat{f}_{3}\left(k_{3}\right) \mathrm{e}_{k_{1}-k_{2}+k_{3}}, \\
& \mathcal{N}_{0}\left(f_{1}, f_{2}, f_{3}\right):=\sum_{k \in \mathbb{Z}} \widehat{f}_{1}(k) \widehat{\widehat{f}_{2}}(k) \widehat{f}_{3}(k) \mathrm{e}_{k} .
\end{aligned}
$$

To solve (1.7), we used the affine ansatz

$$
v(t)=S_{\alpha}(t) \phi^{\omega}+w(t),
$$

where $S_{\alpha}(t)=\mathrm{e}^{i t\left|D_{x}\right|^{\alpha}}$ is the linear propagator. It turns out that the Duhamel's integration of the first Picard's iteration $\mathcal{I N}\left(S_{\alpha}(t) \phi^{\omega}\right)$ has the spatial regularity $H^{\frac{3(\alpha-1)}{2}}-$. Yet, if we place it into the $X^{s, b}$ space, it is bounded a.s. in $X^{(\alpha-1)-, \frac{1}{2}+}$. In both these spaces, the spatial regularity for the first Picard's iteration is better than the initial data which merely lives in $H^{\frac{\alpha-1}{2}}$-. In order to close the fix-point argument, we should place the error term into some $X^{s, \frac{1}{2}+}$ space. Due to the weak dispersive effect, when $\alpha<2$, it was proved in [13] (see also [28]) that the Duhamel's integration of the tri-linear operator is bounded on $X^{s, \frac{1}{2}+}$ only if $s \geq \frac{1}{2}-\frac{\alpha}{4}$. Therefore, this affine decomposition ansatz is suitable in $X^{s, b}$ type space only if $\alpha-1>\frac{1}{2}-\frac{\alpha}{4}$ which gives us the constraint $\alpha>\frac{6}{5}$.

Even if we do not place the first Picard's iteration in the $X^{s, b}$ type space, the other place that gives us the constraint $\alpha>\frac{6}{5}$ is the high-low-low frequency interaction for the crossing terms of the form

$$
f_{N}:=\mathcal{I N}\left(S_{\alpha}(t) \mathbf{P}_{N} \phi^{\omega}, \mathbf{P}_{\ll N} w, \mathbf{P}_{\ll N} w\right) .
$$

By ignoring the issue of the modulation, the above term can be written formally as

$$
\sum_{\substack{k, k_{1}, k_{2}, k_{3} \\ k_{1}-k_{2}+k_{3}=k}} \mathbf{1}_{\left|k_{1}\right| \sim N,\left|k_{2}\right|,\left|k_{3}\right| \ll N} \mathbf{1}_{\left|k_{1}\right|^{\alpha}-\left|k_{2}\right|^{\alpha}+\left|k_{3}\right|^{\alpha}-|k|^{\alpha}=O(1)} \frac{g_{k_{1}}}{\left[k_{1}\right]^{\frac{\alpha}{2}}} \widehat{w}\left(k_{2}\right) \widehat{w}\left(k_{3}\right) .
$$

From a counting argument, the $X^{s, b}$ norm of the above quantity can be bounded by

$$
N^{(s-(\alpha-1))-}\left\|\widehat{w}_{2}\right\|_{l^{2}}\left\|\widehat{w}_{3}\right\|_{l^{2}},
$$

hence we should require $s<\alpha-1$ to ensure that the above expression is bounded. It turns out that this high-low-low frequency interaction is the most singular part in the analysis. In order to improve the constraint of $\alpha$, a better understanding of this singular part is necessary.

1.5. Refined resolution ansatz. Refined resolution ansatz to treat the singular high-low type interaction has been recently introduced by Bringmann [8] for the wave equation and by Deng-Nahmod-Yue [17] for the 2D NLS in very different ways. The common feature in both these work is the observation that the low frequency component is independent with the high frequency linear evolution and the most singular interactions (high-low type) are removed by viewing them as part of the linear evolution for the high-frequency data and isolating them from $w(t)$ in the previous affine ansatz $u(t)=S_{\alpha}(t) \phi^{\omega}+w(t)$. More importantly, the authors in [17] exploits the fact that the low frequency components are also random, and this randomness of low frequency components is exactly what is captured by the matrix/operator norms introduced there. To better explain the idea in the context of FNLS, we need to introduce an extra term $\zeta$ such that $\Psi=S_{\alpha}(t) \phi^{\omega}+\zeta$ solves

$$
i \partial_{t} \Psi+\left|D_{x}\right|^{\alpha} \Psi=\mathcal{I N}\left(\mathbf{P}_{\text {high }} \Psi, \mathbf{P}_{\text {low }} u, \mathbf{P}_{\text {low }} u\right),\left.\quad \Psi\right|_{t=0}=\mathbf{P}_{\text {high }} \phi^{\omega} .
$$

Through this decomposition, on the one hand, the new remainder will solve some nonlinear equation with essentially no high-low-low type frequency interaction. On the other hand, since the isolated singular part $\Psi$ solves roughly a linear transport equation with some "potential" independent of the high frequency initial data, it will inherit the randomness 
from the initial data $\phi^{\omega}$. Though $\Psi$ is no more regular than $X^{(\alpha-1)-, \frac{1}{2}+}$ in general, it has its own random structure though captured by certain matrix-norms.

Now we recall the precise resolution ansatz of [17] in our context. Set $y_{N}=v_{N}-v_{\frac{N}{2}}$. Then $y_{N}$ solves the equation

$$
\left\{\begin{array}{l}
\left(i \partial_{t}+\left|D_{x}\right|^{\alpha}\right) y_{N}=\mathcal{N}\left(v_{\frac{N}{2}}+y_{N}\right)-\mathcal{N}\left(v_{\frac{N}{2}}\right) \\
\left.y_{N}\right|_{t=0}=\mathbf{P}_{N} \phi^{\omega}
\end{array}\right.
$$

For fixed $N$, we denote by $L_{N}$ the largest dyadic number $L$ such that $L<N^{1-\delta}$. For $L \leq$ $L_{N}$, we introduce the function $\psi_{L}^{N}$ which captures the high-low-low frequency interaction:

$$
\left\{\begin{array}{l}
\left(i \partial_{t}+\left|D_{x}\right|^{\alpha}\right) \psi_{L}^{N}=-2 \Pi_{N} \mathcal{N}_{3}\left(\psi_{L}^{N}, \Pi_{L} v_{L}, \Pi_{L} v_{L}\right) \\
\left.\psi_{L}^{N}\right|_{t=0}=\mathbf{P}_{N} \phi^{\omega}
\end{array}\right.
$$

When $L=\frac{1}{2}$, we define $\psi_{\frac{1}{2}}^{N}:=S_{\alpha}(t) \mathbf{P}_{N} \phi^{\omega}$. Set $w_{N}=y_{N}-\psi_{L_{N}}^{N}$, then $w_{N}$ solves the equation

$$
\left\{\begin{array}{l}
\left(i \partial_{t}+\left|D_{x}\right|^{\alpha}\right) w_{N}=\mathcal{N}\left(w_{N}+\psi_{L_{N}}^{N}+v_{\frac{N}{2}}\right)-\mathcal{N}\left(v_{\frac{N}{2}}\right)+2 \Pi_{N} \mathcal{N}_{3}\left(\psi_{L_{N}}^{N}, \Pi_{L_{N}} v_{L_{N}}, \Pi_{L_{N}} v_{L_{N}}\right), \\
\left.w_{N}\right|_{t=0}=0 .
\end{array}\right.
$$

Denote by $f_{N}=\psi_{\frac{1}{2}}^{N}$ the free evolution part and $\zeta_{L}^{N}=\psi_{L}^{N}-\psi_{\frac{L}{2}}^{N}$ if $\frac{1}{2}<L \leq L_{N}, \zeta_{\frac{1}{2}}^{N}=0$. Then

$$
y_{N}(t)=f_{N}(t)+\sum_{\frac{1}{2}<L \leq L_{N}} \zeta_{L}^{N}(t)+w_{N}(t)
$$

and the full resolution ansatz is

$$
v(t)=S_{\alpha}(t) \phi^{\omega}+\sum_{N} \sum_{\frac{1}{2}<L \leq L_{N}} \zeta_{L}^{N}(t)+\sum_{N} w_{N}(t) .
$$

Remark 1.3. $v_{\frac{N}{2}}-v_{L_{N}}$ is pretended to have frequencies greater than $L_{N}$ and $w_{N}$ is pretended to have frequencies comparable to $N$, and $\zeta_{L}^{N}$ is pretended to contain the portion of frequency interaction from $(\sim N) \times(\sim L) \times(\lesssim L)$. Therefore, by expanding the right hand side of (1.10), all the multi-linear forms essentially do not have bad frequency interaction of the form $(\sim N) \times\left(\ll L_{N}\right) \times\left(\ll L_{N}\right)$.

The second parameter $L$ quantifies the range of "low-frequency" perturbation for the linear evolution of the high frequency data. It can be viewed as a deformation from the random oscillation effect to the time-oscillation effect (dispersive effect). When $L$ is relatively small, $\zeta_{L}^{N}$ behaves like the first Picard iteration of the linear evolution of Gaussian variables whose random effect is dominant. When $L$ is relatively large, $\zeta_{L}^{N}$ behaves like the error $w_{N}$ whose $X^{0, b}$-regularity is much better.

Structure of $\psi_{L}^{N}$ in terms of operators. Given $v_{L}$, the equation of $\psi_{L}^{N}$ is linear with respect to the initial data. Therefore, we can write

$$
\psi_{L}^{N}=\mathcal{H}^{N, L}\left(\mathbf{P}_{N} \phi^{\omega}\right) .
$$

The operator $\mathcal{H}^{N, L}$ is the random averaging operator and $\mathcal{F}_{x} \mathcal{H}^{N, L} \mathcal{F}_{x}^{-1}$ has kernel $\left(H_{k k *}^{N, L}(t)\right)$, thus we have

$$
\widehat{\psi_{L}^{N}}(t, k)=\sum_{\frac{N}{2}<\left|k^{*}\right| \leq N} H_{k k^{*}}^{N, L}(t) \frac{g_{k^{*}}(\omega)}{\left[k^{*}\right]^{\frac{\alpha}{2}}} .
$$

In other words, $H_{k k^{*}}^{N, L}(t)$ is the $k$-th Fourier mode of the solution to

$$
\left\{\begin{array}{l}
\left(i \partial_{t}+\left|D_{x}\right|^{\alpha}\right) \varphi=-2 \Pi_{N} \mathcal{N}_{3}\left(\varphi, \Pi_{L} v_{L}, \Pi_{L} v_{L}\right), \\
\left.\varphi\right|_{t=0}=\mathbf{1}_{\frac{N}{2}<\left|k^{*}\right| \leq N} \mathrm{e}_{k^{*}}
\end{array}\right.
$$


Obviously,

$$
\operatorname{supp}_{k, k^{*}}\left(H_{k, k *}^{N, L}\right) \subset\left\{\left(k, k^{*}\right):|k| \leq N, \frac{N}{2}<\left|k^{*}\right| \leq N\right\} .
$$

When $L=\frac{1}{2}$, we use the convention $H_{k k^{*}}^{N, \frac{1}{2}}=\mathrm{e}^{i t|k|^{\alpha}} \mathbf{1}_{k=k^{*}}$. Similarly, we denote by $h^{N, L}=\mathcal{H}^{N, L}-\mathcal{H}^{N, \frac{L}{2}}$, hence $\zeta_{L}^{N}=h^{N, L}\left(\mathbf{P}_{N} \phi^{\omega}\right)$. The kernel of $\mathcal{F}_{x} h^{N, L} \mathcal{F}_{x}^{-1}$ is denoted by $\left(h_{k k^{*}}^{N, L}\right)$ and $h_{k k^{*}}^{N, L}$ has the same $k, k^{*}$ support property as $H_{k k^{*}}^{N, L}$. The key point here is that $H_{k k^{*}}^{N, L}, h_{k k^{*}}^{N, L}$ belong to the Borel $\sigma$-algebra $\mathcal{B}_{\leq L}$ generated by $\left\{g_{k}(\omega):|k| \leq L\right\}$, hence $H_{k k^{*}}^{N, L}, h_{k k^{*}}^{N, L}$ are independent of $\sigma$-algebra $\mathcal{B}_{>\frac{N}{2}}$ generated by $\left\{g_{k}(\omega):|k|>N / 2\right\}$. The random oscillation effect will be captured in terms of suitable norms for the operators $\mathcal{H}^{N, L}, h^{N, L}$, as explained in [17]. In this article, we need the Hilbert-Schmidt type norm to capture the $X^{s, b}$-regularity as well as the $L^{\infty}$ size of $\psi_{L}^{N}$ and a Fourier-Lebesgue type norm to measure the size of the Fourier-coefficients of $\psi_{L}^{N}$.

Probabilistic local convergence. To prove Theorem 2, the key point is a local convergence result for dyadic sequences which we will describe below:

Theorem 3. Let $\alpha>\alpha_{0}$. Then there exist $C_{0}>0$ and sufficiently small numbers $\theta>$ $0, \epsilon>0$, such that for each sufficiently small $T>0$, there exists a set $\Omega_{T} \subset \Omega$ with the following properties:

(i) $\mathbb{P}\left[\Omega_{T}^{c}\right]<C_{0} \mathrm{e}^{-T^{-\theta}}$.

(ii) $\forall \omega \in \Omega_{T}$, the sequence of unique smooth solutions $\left(v_{N}\right)_{N \in 2^{\mathbb{N}}}$ of

$$
i \partial_{t} v_{N}+\left|D_{x}\right|^{\alpha} v_{N}=\mathcal{N}\left(v_{N}\right)
$$

with initial data $\left.v_{N}\right|_{t=0}=\Pi_{N} \phi^{\omega}$ given by $(1.2)$ is a Cauchy sequence in $C\left([-T, T] ; H^{\sigma_{0}}(\mathbb{T})\right)$. More precisely, for all $|t| \leq T$ and $N, v_{N}$ admits a decomposition

$$
v_{N}(t)=S(t) \Pi_{N} \phi^{\omega}+\zeta^{N}+W_{N}, \text { where } \zeta^{N}:=\sum_{M \leq N} \sum_{\frac{1}{2}<L \leq L_{N}} \zeta_{L}^{N}
$$

with the property that $\left(W_{N}\right)_{N \in 2^{\mathbb{N}}}$ is a Cauchy sequence in $X_{T}^{\frac{1}{2}-\frac{\alpha}{4}+\epsilon, \frac{1}{2}+\epsilon}$ and for each $N, L$,

$$
\begin{gathered}
\left\|\zeta_{L}^{N}\right\|_{L_{t}^{4}\left([-T, T] ; L^{\infty}(\mathbb{T})\right)} \leq C_{0} N^{-(\alpha-1)-\epsilon} L^{\frac{1}{2}-\nu}, \\
\left\|\zeta_{L}^{N}\right\|_{L^{\infty}\left([-T, T] ; H^{(\alpha-1)-\epsilon}(\mathbb{T})\right)} \leq C_{0} L^{-\nu} N^{-\frac{\epsilon}{2}} \\
\left\|\zeta_{L}^{N}\right\|_{L^{\infty}\left([-T, T] ; \mathcal{F} L^{\frac{\alpha}{2}-\epsilon, \infty}(\mathbb{T})\right)} \leq C_{0} L^{-\nu} N^{-\frac{\epsilon}{2}}
\end{gathered}
$$

with $\nu=\min \left\{\frac{1}{2}-\frac{\alpha}{4}, \frac{7(\alpha-1)}{4}\right\}-\epsilon$.

(iii) The sequence $\left(v_{n}\right)_{n \in \mathbb{N}}$ of unique smooth solutions of

$$
i \partial_{t} v_{n}+\left|D_{x}\right|^{\alpha} v_{n}=\mathcal{N}\left(v_{n}\right),\left.\quad v_{n}\right|_{t=0}=\Pi_{n} \phi^{\omega}
$$

converges in $C\left([-T, T] ; H^{\sigma_{0}}(\mathbb{T})\right)$.

Note that by undoing the gauge transform

$$
u_{n}(t)=v_{n}(t) \mathrm{e}^{-\frac{i t}{\pi} \int_{\mathbb{T}}\left|v_{n}\right|^{2} d x},
$$

the unique solution $u_{n} \in C\left([-T, T] ; H^{\sigma_{0}}(\mathbb{T})\right)$ of the original FNLS equation is also a Cauchy sequence in $C\left([-T, T] ; H^{\sigma_{0}}(\mathbb{T})\right)$, which proves Theorem 2 (locally in time). Unlike [17. where the dispersive effect is very strong while the nonlinearity can be arbitrarily large, we deal with the NLS model with a fixed nonlinearity but with very weak dispersion. Another different feature is that we do not need to renormalize the equation which makes the problem more natural from a purely PDE perspective. Therefore the type of probabilistic well-posedness we get in this paper is close in spirit to the line of research initiated by Burq and the second author in [11, 12. More importantly, we perform the 
multi-linear estimates in a very different manner compared with [17]. Indeed, in [17], all the analysis was performed in the Fourier space, thanks to the strong linear and multilinear smoothing effect. However, in our situation, the deterministic smoothing is very weak (for Strichartz we loose almost $\frac{1}{8}$ derivative) and we rely more on the linear random oscillation effect. It is at this point that we need to define an extra Fourier-Lebesgue type operator norm $S^{b, q}$ in Section 3 .

We believe that the constraint $\alpha>\alpha_{0}=\frac{31-\sqrt{233}}{14}$ is technical. We point out again that $\alpha_{0}<\frac{8}{7}$ where $\frac{8}{7}$ is the threshold for the constraint $\frac{1}{2}-\frac{\alpha}{4}>\frac{3(\alpha-1)}{2}$ and $H^{\frac{3(\alpha-1)}{2}}-$ is the regularity of the first Picard's iteration $\mathcal{I N}\left(S_{\alpha}(t) \phi^{\omega}\right)$. The technical constraint $\alpha>\alpha_{0}$ is mainly caused by the condition $\nu<\frac{7(\alpha-1)}{4}$. Indeed, this comes from the upper bound $N^{-(\alpha-1)+} L^{-\frac{7(\alpha-1)}{4}+}$ of the $X^{0, b}$ norm of the expression

$$
\mathcal{I N}\left(\mathbf{P}_{N} S_{\alpha}(t) \phi, \mathbf{P}_{L} S_{\alpha}(t) \phi+\zeta_{R}^{L}, \mathbf{P}_{L} S_{\alpha}(t) \phi+\zeta_{R}^{L}\right)
$$

Note that $\zeta_{R}^{L}$ can be viewed as a size $R$ perturbation of Gaussians with Fourier support $~$ $L$. Compared with the expression $\mathcal{I N}\left(\mathbf{P}_{N} S_{\alpha}(t) \phi, \mathbf{P}_{L} S_{\alpha}(t) \phi, \mathbf{P}_{L} S_{\alpha}(t) \phi\right)$, the non-resonant relation $k_{2} \neq k_{1}, k_{3}$ will be destroyed and consequently, the estimate for terms like

$$
\mathcal{I N}\left(\mathbf{P}_{N} S_{\alpha}(t) \phi, \zeta_{R}^{N}, \zeta_{R}^{L}\right), \quad \mathcal{I N}\left(\mathbf{P}_{N} S_{\alpha}(t) \phi, \mathbf{P}_{L} S_{\alpha}(t) \phi, \zeta_{R}^{L}\right)
$$

is worse than the former 1 .

Refined resolution ansatz in the context of nonlinear PDE in the presence of singular randomness were used in many previous works. In [1], 27] ansatz taking contributions from possibly infinitely many Picard iterations are introduced. In [22], [20], in the context of parabolic equations, resolution ansatz exploiting randomness structure of certain terms beyond the affine ansatz are introduced (the randomness is captured using certain linearisation operators). This type of ansatz was first introduced in the context of dispersive PDE in [21] and further developed in [9, 25]. Different ansatz, which involve the randomness structure of operators and tensors, are introduced in [17], [18]. It should be underlined that all these contributions are extensions of the ideas introduced in the fundamental papers by Bourgain [2, 3, 4.

Organization of the article. In this article, we only address the proof of Theorem 3 since the remaining arguments of the proof of Theorem 2 follow from [28]. In Section 2, we recall some preliminaries and define the functional spaces for functions and operators. In Section 3, following the iterative scheme in [17, we first reduce the proof of Theorem 3 to an induction statement (Proposition 3.3). Then by assuming key multi-linear estimates summarized in Proposition 3.7, we prove the induction Proposition 3.3. The remaining sections are devoted to the proof of the statements in Proposition 3.7. In Section 4, we deduce the $L^{\infty}$ and Fourier-Lebesgue property for the "paracontrolled" terms which will be used intensively. Next in Section 5, we prove the mapping properties of the random averaging operators leading to the self-closeness of the fix-point problem for $h^{N, L}$. Then in Section 6 , we reduce the key multi-linear operators to the low-modulation cases in order to focus only on the discrete multi-linear summations later. In Section 7, we prove the bilinear estimates for the kernels of random averaging operators which helps us to control the source term of the fix-point problem for $h^{N, L}$. Finally in the remaining sections, we focus on the tri-linear estimates used to close the fix-point problem for the error $w_{N}$, in different frequency interactions regimes. In all multi-linear estimates, we always describe available algorithms first and then do the case-by-case analysis by implementing the algorithms.

Acknowledgment. We thank Tadahiro Oh for interesting discussions while the first author visiting the University of Edinburgh. We thank Yu Deng for valuable comments on the first version of the manuscript. The authors are supported by the ANR grant ODA (ANR-18-CE40- 0020-01).

\footnotetext{
${ }^{1}$ See (v) of Lemma 7.5 and (ii) of Lemma 7.6
} 


\section{Notations And PRELiminaries}

2.1. General notations. The capital numbers $N, M, L, R$ represent dyadic numbers greater than $\frac{1}{2}$. For a finite collection of dyadic numbers $\left\{N_{1}, N_{2}, \cdot, N_{k}\right\}$, we denote by $N_{(1)} \geq$ $N_{(2)} \geq N_{(3)} \geq \cdots N_{(k)}$ be the non-increasing rearrangement of it.

For two quantities $A, B$, the asymptotic notation $A \lesssim B(A \gtrsim B)$ means that there exists a constant $C$ such that $A \leq C B(A \geq C B)$. The notation $A \sim B$ means that $A \lesssim B$ and $A \gtrsim B$. The notation $A \lesssim_{X} B\left(A \gtrsim_{X} B\right)$ is used to specify that the constant $C$ depends on $X$.

For the Lebesgue exponents $1 \leq p, q, r \leq \infty$, we always use $p^{\prime}, q^{\prime}, r^{\prime}$ to denote their conjugate exponents such that $\frac{1}{p}+\frac{1}{p^{\prime}}=1$ with the canonical modification when $p=1$ or $\infty$. For $1 \leq j \leq n$, denote by $\left(Z_{j}, \mu_{j}\right)$ a finite sequence of measure space with the standard $L^{p_{j}}$ norm

$$
\left\|f\left(z_{j}\right)\right\|_{L_{z_{j}}^{p_{j}}}:=\left(\int_{Z_{j}}\left|f\left(z_{j}\right)\right|^{p_{j}} d \mu_{j}\right)^{\frac{1}{p_{j}}} .
$$

We will simply denote by $L_{z_{1}}^{p_{1}} L_{z_{2}}^{p_{2}} \cdots L_{z_{j}}^{p_{j}}$ to stand for $L^{p_{1}}\left(Z_{1} ; L^{p_{2}}\left(Z_{2} ; \cdots ; L^{p_{j}}\left(Z_{j}\right) \cdots\right)\right)$. For example, we denote by $L_{t}^{q} L_{x}^{r} l_{k}^{q}$ to stand for $L^{q}\left(\mathbb{R}_{t} ; L^{r}\left(\mathbb{T}_{x} ; l^{q}(\mathbb{Z})\right)\right)$. The Fourier-Lebesgue space $\mathcal{F} L^{s, q}(\mathbb{T})$ is defined via the norm

$$
\|f\|_{\mathcal{F} L^{s, q}}:=\left\|\langle k\rangle^{s} \widehat{f}(k)\right\|_{l_{k}^{q}} .
$$

We denote by $\Pi_{N}:=\mathcal{F}_{x}^{-1} \mathbf{1}_{|k| \leq N} \mathcal{F}_{x}$, and $\mathbf{P}_{N}:=\mathcal{F}_{x}^{-1} \mathbf{1}_{N / 2<|k| \leq N} \mathcal{F}_{x}$ if $N \geq 1$ and $\mathbf{P}_{2^{-1}}=$ $\Pi_{2^{-1}} . S_{\alpha}(t)=e^{i t\left|D_{x}\right|^{\alpha}}$. The twisted spacetime Fourier transform is defined as

$$
\widetilde{u}(\lambda, k):=\left(\mathcal{F}_{t, x} u\right)\left(\lambda+|k|^{\alpha}, k\right) .
$$

We also denote by

$$
\widetilde{h}_{k k^{*}}(\lambda):=\left(\mathcal{F}_{t} h_{k k^{*}}\right)\left(\lambda+|k|^{\alpha}\right), \quad \widetilde{\Theta}_{k k^{\prime}}\left(\lambda, \lambda^{\prime}\right)=2 \pi\left(\mathcal{F}_{t, t^{\prime}} \Theta_{k k^{\prime}}\right)\left(\lambda+|k|^{\alpha},-\lambda^{\prime}-\left|k^{\prime}\right|^{\alpha}\right) .
$$

The definition of $\widetilde{\Theta}_{k k^{\prime}}\left(\lambda, \lambda^{\prime}\right)$ is such that if a operator is given by

$$
\mathcal{Q}(w)(t, k)=\sum_{k^{\prime}} \int \Theta_{k k^{\prime}}\left(t, t^{\prime}\right)\left(\mathcal{F}_{x} w\right)\left(t^{\prime}, k^{\prime}\right) d t^{\prime},
$$

then

$$
\widetilde{\mathcal{Q}}(w)(\lambda, k)=\sum_{k^{\prime}} \int \widetilde{\Theta}_{k k^{\prime}}\left(\lambda, \lambda^{\prime}\right) \widetilde{w}\left(\lambda^{\prime}, k^{\prime}\right) d \lambda^{\prime} .
$$

Define the affine space for a given number $k \in \mathbb{Z}$

$$
\Gamma(k):=\left\{\left(k_{1}, k_{2}, k_{3}\right) \in \mathbb{Z}^{3}: k_{2} \neq k_{1}, k_{2} \neq k_{3}, k_{1}-k_{2}+k_{3}=k\right\},
$$

and the resonant function on $\Gamma(k)$

$$
\Phi_{k_{1}, k_{2}, k_{3}}:=\left|k_{1}\right|^{\alpha}-\left|k_{2}\right|^{\alpha}+\left|k_{3}\right|^{\alpha}-|k|^{\alpha} .
$$

2.2. Spaces for functions and operators. Denote by $S_{\alpha}(t)=\mathrm{e}^{i t\left|D_{x}\right|^{\alpha}}$. Recall that the Fourier restriction type space $X^{s, b}$ is defined with the associated norm

$$
\|u\|_{X^{s, b}}:=\left\|S_{\alpha}(-t) u\right\|_{H^{s}\left(\mathbb{T}_{x} ; H^{b}\left(\mathbb{R}_{t}\right)\right)}=\left\|\langle\lambda\rangle^{b}\langle k\rangle^{s} \widetilde{u}(\lambda, k)\right\|_{l_{k}^{2} L_{\lambda}^{2}} .
$$

Similarly, the Fourier-Lebesgue restriction space $X_{p, q}^{s, \gamma}$ is defined via the norm

$$
\|u\|_{X_{p, q}^{s, \gamma}}:=\left\|\langle\lambda\rangle^{\gamma}\langle k\rangle^{s} \widetilde{u}(\lambda, k)\right\|_{l_{k}^{p} L_{\lambda}^{q}} .
$$

Note that $X_{2,2}^{s, b}=X^{s, b}$. For finite time interval $I \subset \mathbb{R}$, the localized restriction space $X_{I}^{s, b}$ is defined via the norm

$$
\|u\|_{X_{I}^{s, b}}:=\inf \left\{\|v\|_{X^{s, b}}:\left.v\right|_{I}=u\right\} .
$$

For $X^{s, b}$ spaces, we have the following statements. 
Lemma 2.1. Let $\chi \in \mathcal{S}(\mathbb{R})$. Then for $0<T<1, s \in \mathbb{R}$ and $-\frac{1}{2}<\widetilde{b} \leq b<\frac{1}{2}$, we have the estimate

$$
\|\chi(t / T) u\|_{X^{s, \tilde{b}}} \lesssim T^{b-\widetilde{b}}\|u\|_{X^{s, b}} .
$$

Moreover, if $\left.u\right|_{t=0}=0$, then the above estimate holds for $0<\widetilde{b} \leq b<1$.

Note that the proof of the last statement can be found as Proposition 2.7 of [17].

Lemma 2.2. Let $\chi \in \mathcal{S}(\mathbb{R})$. Then for $s \in \mathbb{R}, \frac{1}{2}<b<1$, we have the estimate

$$
\left\|\chi(t) \int_{0}^{t} S_{\alpha}\left(t-t^{\prime}\right) F\left(t^{\prime}\right) d t^{\prime}\right\|_{X^{s, b}} \lesssim\|F\|_{X^{s, b-1}} .
$$

For $\mathcal{H}(t)$, time-dependent linear operator on $l^{2}$ with kernel $\left(H_{k k^{*}}(t)\right)$, we introduce the norms:

$$
\begin{aligned}
\|\mathcal{H}\|_{Y^{b}} & :=\left\|\langle\lambda\rangle^{b} \widetilde{H}_{k k^{*}}(\lambda)\right\|_{l_{k^{*}}^{2} \rightarrow L_{\lambda}^{2} l_{k}^{2}}, \\
\|\mathcal{H}\|_{Z^{b}} & :=\left\|\langle\lambda\rangle^{b} \widetilde{H}_{k k^{*}}(\lambda)\right\|_{L_{\lambda}^{2} l_{k, k^{*}}^{2}}, \\
\|\mathcal{H}\|_{S^{b, q}} & :=\left\|\langle\lambda\rangle^{\frac{2 b}{q^{\prime}}} \widetilde{H}_{k k^{*}}(\lambda)\right\|_{l_{k}^{\infty} L_{\lambda}^{q} l_{k^{*}}^{2}},
\end{aligned}
$$

where $\frac{1}{q}+\frac{1}{q^{\prime}}=1,1 \leq q \leq \infty$. For a linear operator $\Theta$ with kernel $\left(\Theta_{k k^{\prime}}\left(t, t^{\prime}\right)\right)$, we introduce the matrix norms:

$$
\begin{aligned}
& \|\Theta\|_{Y^{b_{1}, b_{2}}}:=\left\|\langle\lambda\rangle^{b_{1}}\left\langle\lambda^{\prime}\right\rangle^{-b_{2}} \widetilde{\Theta}_{k k^{\prime}}\left(\lambda, \lambda^{\prime}\right)\right\|_{L_{\lambda^{\prime}}^{2} l_{k}^{\prime 2} \rightarrow L_{\lambda}^{2} l_{k}^{2},} \\
& \|\Theta\|_{Z^{b_{1}, b_{2}}}:=\left\|\langle\lambda\rangle^{b_{1}}\left\langle\lambda^{\prime}\right\rangle^{-b_{2}} \widetilde{\Theta}_{k k^{\prime}}\left(\lambda, \lambda^{\prime}\right)\right\|_{L_{\lambda, \lambda^{\prime}}^{2} l_{k, k^{\prime}}^{2}}, \\
& \|\Theta\|_{S^{b_{1}, b_{2}, q}}:=\left\|\langle\lambda\rangle^{\frac{2 b_{1}}{q^{\prime}}}\left\langle\lambda^{\prime}\right\rangle^{-b_{2}} \widetilde{\Theta}_{k k^{\prime}}\left(\lambda, \lambda^{\prime}\right)\right\|_{l_{k}^{\infty} L_{\lambda}^{q} L_{\lambda^{\prime}}^{2} l_{k^{\prime}}^{2}} .
\end{aligned}
$$

Note that when we ignore the $k^{*}$ variable, the $S^{b, q}$ norm is just the restricted-type FourierLebesgue norm $X_{\infty, q}^{0, \gamma}$ with $\gamma=\frac{2 b}{q^{\prime}}$. The reason for introducing of the space $S^{b, q}$ is two-fold. First it characterizes the Fourier-Lebesgue norm of the para-controlled term $\psi_{L}^{N}$ which is morally $N^{-\frac{\alpha}{2}}$ for small $L$. This allows us to carry out many multi-linear estimates simply by Cauchy-Schwartz, as in our previous work [28]. The second reason is technical. When we do the Wiener chaos estimate, in almost all the situations, leaving out $\left\|h^{N, L}\right\|_{S^{b, q}}$ is better than leaving out $\left\|h^{N, L}\right\|_{Z^{b}}$ since the later losses $N^{1-\frac{\alpha}{2}}$ factor.

Sometimes we will abuse the notation and write simply

$$
\left\|\Theta_{k k^{\prime}}\left(\lambda, \lambda^{\prime}\right) \cdot m\left(k, k^{\prime}\right)\right\|_{\mathcal{X}^{b_{1}, b_{2}}}:=\left\|\mathcal{F}_{t, x}^{-1}\left(\widetilde{\Theta}_{k k^{\prime}}\left(\lambda, \lambda^{\prime}\right) m\left(k, k^{\prime}\right)\right)\right\|_{\mathcal{X}^{b_{1}, b_{2}}},
$$

for $\mathcal{X}=Y, Z$ or $S$.

Lemma 2.3. Let $\chi \in \mathcal{S}(\mathbb{R})$, and recall that $\chi_{T}(t):=\chi\left(T^{-1} t\right)$ for $0<T \ll 1$. Then for $u(t, x)$ and operator $\Theta\left(t, t^{\prime}\right)=\left(\Theta_{k k^{\prime}}\left(t, t^{\prime}\right)\right)$ satisfying $u(t=0, \cdot)=0, \Theta\left(t=0, t^{\prime}\right)=0$, we have

$$
\left\|\chi_{T}(t) u\right\|_{X_{\infty, q}^{0, \gamma}} \lesssim T^{\gamma_{1}-\gamma}\|u\|_{X_{\infty, q}^{0, \gamma_{1}}}, \quad\left\|\chi_{T}(t) \Theta\right\|_{S^{b, q}} \lesssim T^{\frac{2\left(b_{1}-b\right)}{q^{\prime}}}\|\Theta\|_{S^{b_{1}, q}},
$$

with $1 \leq q<\infty, 0<\gamma<\gamma_{1}<1+\frac{1}{q^{\prime}}$, and $0<b<b_{1}<1$.

Proof. The proof is essentially the same as in [17. We present a proof in the appendix.

Fix a time cutoff $\chi \in C_{c}^{\infty}((-1,1))$, we define the time truncated Duhamel operator

$$
\mathcal{I} F(t):=\chi(t) \int_{0}^{t} S_{\alpha}\left(t-t^{\prime}\right)\left(\chi\left(t^{\prime}\right) F\left(t^{\prime}\right)\right) d t^{\prime} .
$$

Lemma 2.4 ([16]). The twisted space-time Fourier transformation is given be

$$
\widetilde{\mathcal{I} F}(\lambda, k)=\int_{\mathbb{R}} K(\lambda, \mu) \widetilde{F}(\mu, k) d \mu,
$$


where

$$
K(\lambda, \mu)=\int_{\mathbb{R}}\left[\frac{\widehat{\chi}(\lambda-\sigma) \widehat{\chi}(\sigma-\mu)}{i \sigma}-\frac{\widehat{\chi}(\lambda) \widehat{\chi}(\sigma-\mu)}{i \sigma}\right] d \sigma .
$$

Moreover, for any $B>1$, we have

$$
|K(\lambda, \mu)| \lesssim_{B}\left(\frac{1}{\langle\lambda\rangle^{B}}+\frac{1}{\langle\lambda-\mu\rangle^{B}}\right) \frac{1}{\langle\mu\rangle} .
$$

We will need an elementary lemma:

Lemma 2.5. Let $0 \leq \sigma \leq \beta$ and $\sigma+\beta>1$. Then for any $\epsilon>0$, we have

$$
\int_{\mathbb{R}} \frac{d y}{\langle y-x\rangle^{\sigma}\langle y\rangle^{\beta}} \lesssim \frac{1}{\langle x\rangle^{\gamma}}
$$

where

$$
\gamma=\left\{\begin{array}{l}
\sigma+\beta-1, \beta<1 \\
\sigma-\epsilon, \beta=1 \\
\sigma, \beta>1
\end{array}\right.
$$

uniformly in $x \in \mathbb{R}$.

Proof. See Lemma 2.2 of [28].

2.3. Counting lemmas and the Strichartz inequality. We need the following elementary counting principle:

Lemma 2.6. Let $I, J$ be two intervals and $\phi$ be a real-valued $C^{1}$ function defined on $I$, then

$$
\#\{k \in I \cap \mathbb{Z}: \phi(k) \in J\} \leq 1+\frac{|J|}{\inf _{\xi \in I}\left|\phi^{\prime}(\xi)\right|} .
$$

Lemma 2.7. Assume that $N \gg N_{2} \vee N_{3}$, then for fixed $k_{2}, k_{3}$ such that $\left|k_{2}\right| \sim N_{2},\left|k_{3}\right| \sim$ $N_{3}$ and $k_{2} \neq k_{3}$, we have

$$
\sum_{\left|k_{1}\right| \sim N} \mathbf{1}_{\Phi_{k_{1}, k_{2}, k_{3}}=\mu+O\left(N^{\epsilon}\right)} \lesssim N^{\epsilon}\left(1+\frac{N^{2-\alpha}}{\left\langle k_{2}-k_{3}\right\rangle}\right),
$$

and the implicit constant is independent of $\mu$.

Proof. This follows from the fact that

$\left|\frac{\partial \Phi_{k_{1}, k_{2}, k_{3}}}{\partial k_{1}}\right|=\left.\alpha\left|\operatorname{sgn}\left(k_{1}\right)\right| k_{1}\right|^{\alpha-1}-\operatorname{sgn}\left(k_{1}-k_{2}+k_{3}\right)\left|k_{1}-k_{2}+k_{3}\right|^{\alpha-1}|\gtrsim| k_{2}-\left.k_{3}|| k_{1}\right|^{\alpha-2}$, if $\left|k_{1}\right| \sim N \gg\left|k_{2}\right|+\left|k_{3}\right|$. We conclude by the elementary counting principal.

Lemma 2.8. Assume that $N_{1} \sim N_{2} \sim N_{3} \sim N$, then for fixed $k_{2}$, $k_{3}$ such that $\left|k_{2}\right| \sim$ $N_{2},\left|k_{3}\right| \sim N_{3}$ and $k_{2} \neq k_{3}$, we have

$$
\sum_{\left|k_{1}\right| \sim N_{1}} \mathbf{1}_{\Phi_{k_{1}, k_{2}, k_{3}}=\mu+O\left(N^{\epsilon}\right)} \lesssim N^{\epsilon}\left(1+\frac{N^{2-\alpha}}{\left\langle k_{2}-k_{3}\right\rangle}\right),
$$

and the implicit constant is independent of $\mu$.

Proof. Arguing as in the proof of the previous lemma, when $\operatorname{sgn}\left(k_{1}\right) \neq \operatorname{sgn}\left(k_{1}-k_{2}+k_{3}\right)$, we have $\left|\partial_{k_{1}} \Phi\right| \sim N^{\alpha-1}$. When $\operatorname{sgn}\left(k_{1}\right)=\operatorname{sgn}\left(k_{1}-k_{2}+k_{3}\right)$, we may assume that $k_{1}>0$, hence

$\left|\frac{\partial \Phi_{k_{1}, k_{2}, k_{3}}}{\partial k_{1}}\right|=\alpha(\alpha-1) \int_{\min \left\{k_{1}, k_{1}-\left(k_{2}-k_{3}\right)\right\}}^{\max \left\{k_{1}, k_{1}-\left(k_{2}-k_{3}\right)\right\}} \frac{d \xi}{|\xi|^{2-\alpha}} \gtrsim\left|k_{2}-k_{3}\right| \min \left\{\frac{1}{\left|k_{1}\right|^{2-\alpha}}, \frac{1}{\left|k_{1}-k_{2}+k_{3}\right|^{2-\alpha}}\right\}$.

This completes the proof of Lemma 2.8 .

We need also the following Lemma proved in [28]. 
Lemma 2.9. Denote by

$$
A_{a, l, M_{1}, M_{2}}:=\left\{k \in \mathbb{Z}: M_{1} \leq|k| \leq 2 M_{1}, M_{2} \leq|a-k| \leq 2 M_{2},\left.|| k\right|^{\alpha}+|a-k|^{\alpha}-l \mid \leq r\right\} .
$$

Then for $r \geq \frac{1}{100}, 1<\alpha<2$, we have

$$
\# A_{a, l, M_{1}, M_{2}}(r) \lesssim \min \left\{M_{1}, M_{2}\right\}^{1-\frac{\alpha}{2}} r^{\frac{1}{2}},
$$

where the implicit constant is independent of $a, l, r, M_{1}$ and $M_{2}$.

Next we recall the following bilinear Strichartz inequality:

Lemma $2.10([28])$. Let $1<\alpha \leq 2$ and $s \geq \frac{1}{2}-\frac{\alpha}{4}$. Then for any $N \geq M$, we have

$$
\left\|\mathbf{P}_{N} f \cdot \mathbf{P}_{M} g\right\|_{L_{t, x}^{2}} \lesssim M^{s}\left\|\mathbf{P}_{N} f\right\|_{X^{0, \frac{3}{8}}}\left\|\mathbf{P}_{M} g\right\|_{X^{0, \frac{3}{8}}} .
$$

\subsection{Estimates for operators.}

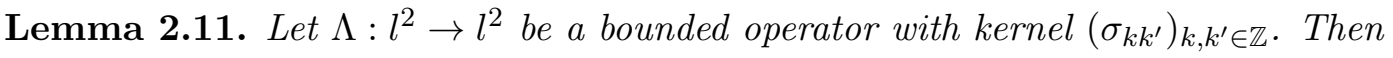

$$
\|\Lambda\|_{l^{2} \rightarrow l^{2}} \leq \sup _{k}\left|\sigma_{k k}\right|+\left(\sum_{k, k^{\prime}: k \neq k^{\prime}}\left|\sigma_{k k^{\prime}}\right|^{2}\right)^{\frac{1}{2}} .
$$

Proof. For any $a \in l^{2}, d \in l^{2}$,

$$
(\Lambda a, d)_{l^{2}}=\sum_{k \neq k^{\prime}} \sigma_{k k^{\prime}} a_{k^{\prime}} \bar{d}_{k}+\sum_{k} \sigma_{k k} a_{k} \bar{d}_{k} .
$$

By Cauchy-Schwartz, we have

$$
\begin{aligned}
\left|(\Lambda a, d)_{l^{2}}\right| & \leq\left(\sum_{k}\left|d_{k}\right|^{2}\right)^{\frac{1}{2}}\left(\sum_{k}\left|\sum_{k, k^{\prime}: k^{\prime} \neq k^{\prime}}\right| \sigma_{k k^{\prime}} \|\left. a_{k^{\prime}}\right|^{2}\right)^{2}+\sup _{k}\left|\sigma_{k k}\right|\|a\|_{l^{2}}\|d\|_{l^{2}} \\
& \leq\left[\sup _{k}\left|\sigma_{k k}\right|+\left(\sum_{k, k^{\prime}: k \neq k^{\prime}}\left|\sigma_{k k^{\prime}}\right|^{2}\right)^{\frac{1}{2}}\right]\|a\|_{l^{2}}\|d\|_{l^{2}} .
\end{aligned}
$$

In view of the duality, this completes the proof of Lemma 2.11 .

The same argument yields:

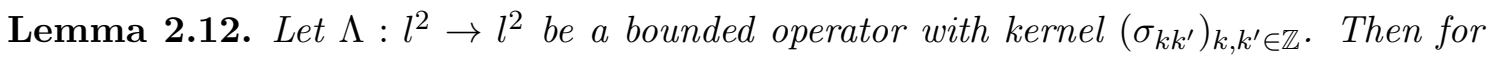
any $L>0$,

$$
\|\Lambda\|_{l^{2} \rightarrow l^{2}} \leq L \sup _{k, k^{\prime}:\left|k-k^{\prime}\right|<L}\left|\sigma_{k, k^{\prime}}\right|+\left(\sum_{k, k^{\prime}:\left|k-k^{\prime}\right| \geq L}\left|\sigma_{k k^{\prime}}\right|^{2}\right)^{\frac{1}{2}} .
$$

Proof. The only difference is the estimate for the quantity

$$
\left|\sum_{k, k^{\prime}:\left|k-k^{\prime}\right|<L} \sigma_{k, k^{\prime}} a_{k} d_{k^{\prime}}\right| \text {. }
$$

We first pull out $\sup _{k, k^{\prime}:\left|k-k^{\prime}\right|<L}\left|\sigma_{k, k^{\prime}}\right|$ and then use Cauchy-Schwartz and Young's convolution inequality to estimate $\sum_{k, k^{\prime}} \mathbf{1}_{\left|k-k^{\prime}\right|<L} a_{k} d_{k^{\prime}}$ as

$$
\|a\|_{l^{2}}\|d\|_{l^{2}}\left\|\mathbf{1}_{|\cdot|<L}\right\|_{l^{1}} \leq L\|a\|_{l^{2}}\|d\|_{l^{2}} .
$$

This completes the proof of Lemma 2.12 .

Lemma 2.13. Let $\mathcal{G}: l_{k}^{1} l_{k_{1}}^{2} \rightarrow l_{k_{2}}^{2}$ is a bounded operator defined via

$$
b_{k, k_{1}} \mapsto \sum_{k_{1}, k} \sigma_{k, k_{1}}^{k_{2}} b_{k, k_{1}} .
$$

Then

$$
\|\mathcal{G}\|_{l_{k}^{1} l_{k_{1}}^{2} \rightarrow l_{k_{2}}^{2}} \leq \sup _{k, k_{1}}\left(\sum_{k_{2}}\left|\sigma_{k, k_{1}}^{k_{2}}\right|^{2}\right)^{\frac{1}{2}}+\sup _{k, k^{\prime}}\left(\sum_{\substack{k_{1}, k_{1}^{\prime} \\\left(k, k_{1}\right) \neq\left(k^{\prime}, k_{1}^{\prime}\right)}}\left|\sum_{k_{2}} \sigma_{k^{\prime}, k_{1}^{\prime}}^{k_{2}} \bar{\sigma}_{k, k_{1}}^{k_{2}}\right|^{2}\right)^{\frac{1}{4}} .
$$


Proof. One verifies directly that $\mathcal{G}^{*}: l_{k_{2}}^{2} \rightarrow l_{k}^{\infty} l_{k_{1}}^{2}$ is given by

$$
a_{k_{2}} \mapsto \sum_{k_{2}} \bar{\sigma}_{k, k_{1}}^{k_{2}} a_{k_{2}}
$$

and the matrix element of $\mathcal{G}^{*} \mathcal{G}$ is

$$
\sigma_{k, k_{1}}^{k^{\prime}, k_{1}^{\prime}}=\sum_{k_{2}} \bar{\sigma}_{k, k_{1}}^{k_{2}} \sigma_{k^{\prime}, k_{1}^{\prime}}^{k_{2}}
$$

For $b \in l_{k}^{1} l_{k_{1}}^{2}, d \in l_{k}^{1} l_{k_{1}}^{2}$, we have

$$
\begin{aligned}
& \left|\left\langle\mathcal{G}^{*} \mathcal{G} b, d\right\rangle\right| \leq\left|\sum_{k, k_{1}} \sigma_{k, k_{1}}^{k, k_{1}} b_{k, k_{1}} d_{k, k_{1}}\right|+\left|\sum_{\substack{k, k_{1}, k^{\prime}, k_{1}^{\prime} \\
\left(k, k_{1}\right) \neq\left(k^{\prime}, k_{1}^{\prime}\right)}} \sigma_{k, k_{1}}^{k^{\prime}, k_{1}^{\prime}} b_{k^{\prime}, k_{1}^{\prime}} d_{k, k_{1}}\right| \\
\leq & \sup _{k, k_{1}}\left|\sigma_{k, k_{1}}^{k, k_{1}}\right| \cdot\left\|b_{k, k_{1}}\right\|_{l_{k}^{1} l_{k_{1}}}\left\|d_{k, k_{1}}\right\|_{l_{k}^{\infty} l_{k_{1}}^{2}}+\sum_{k^{\prime}, k}\left(\sum_{\substack{k_{1}, k_{1}^{\prime} \\
\left(k^{\prime}, k_{1}^{\prime}\right) \neq\left(k, k_{1}\right)}}\left|\sigma_{k, k_{1}}^{k^{\prime}, k_{1}^{\prime}}\right|^{2}\right)^{\frac{1}{2}}\left(\sum_{k_{1}, k_{1}^{\prime}}\left|b_{k^{\prime}, k_{1}^{\prime}}\right|^{2}\left|d_{k, k_{1}}\right|^{2}\right)^{\frac{1}{2}} \\
\leq & \sup _{k, k_{1}}\left|\sigma_{k, k_{1}}^{k, k_{1}}\right| \cdot\left\|b_{k, k_{1}}\right\|_{l_{k}^{1} l_{k_{1}}^{2}}\left\|d_{k, k_{1}}\right\|_{l_{k}^{\infty} l_{k_{1}}^{2}}+\sup _{k^{\prime}, k}\left(\sum_{\substack{k_{1}, k_{1}^{\prime} \\
\left(k^{\prime}, k_{1}^{\prime}\right) \neq\left(k, k_{1}\right)}}\left|\sigma_{k, k_{1}}^{k^{\prime}, k_{1}^{\prime}}\right|^{2}\right)^{\frac{1}{2}} \cdot\left\|b_{k^{\prime}, k_{1}^{\prime}}\right\|_{l_{k^{\prime}} l_{k_{1}^{\prime}}^{2}}\left\|d_{k, k_{1}}\right\|_{l_{k}^{1} l_{k_{1}}^{2}} .
\end{aligned}
$$

Using the fact that $l^{1} \hookrightarrow l^{\infty}$, this implies that

$$
\left\|\mathcal{G}^{*} \mathcal{G}\right\|_{l_{k}^{1} l_{k_{1}}^{2} \rightarrow l_{k}^{\infty} l_{k_{1}}^{2}} \leq \sup _{k, k_{1}} \sum_{k_{2}}\left|\sigma_{k, k_{1}}^{k_{2}}\right|^{2}+\sup _{k, k^{\prime}}\left(\sum_{\substack{k_{1}, k_{1}^{\prime} \\\left(k^{\prime}, k_{1}^{\prime}\right) \neq\left(k, k_{1}\right)}}\left|\sum_{k_{2}} \sigma_{k^{\prime}, k_{1}^{\prime}}^{k_{2}} \bar{\sigma}_{k, k_{1}}^{k_{2}}\right|^{2}\right)^{\frac{1}{2}}
$$

From $\|\mathcal{G}\|_{l_{k}^{1} l_{k_{1}}^{2} \rightarrow l_{k_{2}}^{2}}=\left\|\mathcal{G}^{*} \mathcal{G}\right\|_{l_{k}^{1} l_{k_{1}}^{2} \rightarrow l_{k}^{\infty} l_{k_{1}}^{2}}^{\frac{1}{2}}$, we complete the proof of Lemma 2.13 .

Given $h(\lambda)$, a $\lambda$-dependent family of linaer operators on $L^{2}(\mathbb{T})$, we may identify it as a $\lambda$-dependent family of linear operators on $l^{2}$ with kernel $\left(h_{k k^{*}}(\lambda)\right)$, where

$$
h_{k k^{*}}(\lambda)=\left(h(\lambda)\left(\mathrm{e}_{k^{*}}\right), \mathrm{e}_{k}\right) .
$$

We will need two technical lemmas (in the proof of Proposition 3.3) concerning some estimates of the kernel related to $h(\lambda)$ :

Lemma 2.14. Let $h$ be a $\lambda$-dependent family of operators with kernel $\left(h_{k k^{*}}(\lambda)\right)$. Consider the operator $\widetilde{\mathcal{H}}$ with kernel

$$
\widetilde{H}_{k k^{*}}(\lambda):=\sum_{k^{\prime}} \int_{\mathbb{R}} \widetilde{\Theta}_{k k^{\prime}}\left(\lambda, \lambda^{\prime}\right) h_{k^{\prime} k^{*}}\left(\lambda^{\prime}\right) d \lambda^{\prime},
$$

where $\widetilde{\Theta}_{k k^{\prime}}\left(\lambda, \lambda^{\prime}\right)$ is supported in $\left|k-k^{\prime}\right| \lesssim L$, then for any $\beta \geq 0$, we have $\left\|\left\langle\frac{\left|k-k^{*}\right|}{L}\right\rangle^{\beta} \widetilde{H}_{k k^{*}}(\lambda)\right\|_{L_{\lambda}^{2} l_{k}^{2} l_{k^{*}}^{2}} \lesssim \beta\left\|\widetilde{\Theta}_{k k^{\prime}}\left(\lambda, \lambda^{\prime}\right)\right\|_{L_{\lambda^{\prime}}^{2} l_{k^{\prime}}^{2} \rightarrow L_{\lambda}^{2} l_{k}^{2}} \cdot\left\|\left\langle\frac{\left|k^{\prime}-k^{*}\right|}{L}\right\rangle^{\beta} h_{k^{\prime} k^{*}}\left(\lambda^{\prime}\right)\right\|_{L_{\lambda^{\prime}}^{2} l_{k^{\prime}}^{2} l_{k^{*}}^{2}}$.

Proof. See Proposition 2.5 of [17].

Lemma 2.15. Consider the operator $\widetilde{\mathcal{H}}$ with kernel

$$
\widetilde{H}_{k k^{*}}(\lambda):=\sum_{k^{\prime}} \int_{\mathbb{R}} \widetilde{\Theta}_{k k^{\prime}}\left(\lambda, \lambda^{\prime}\right) h_{k^{\prime} k^{*}}\left(\lambda^{\prime}\right) d \lambda^{\prime},
$$

then we have

(i) $\quad\left\|\widetilde{H}_{k k^{*}}(\lambda)\right\|_{l_{k}^{\infty} L_{\lambda}^{q} l_{k^{*}}^{2}} \lesssim\left\|\widetilde{\Theta}_{k k^{\prime}}\left(\lambda, \lambda^{\prime}\right)\right\|_{l_{k}^{\infty} L_{\lambda}^{q} L_{\lambda^{\prime}}^{2} l_{k^{\prime}}^{2}}\left\|h_{k^{\prime} k^{*}}\left(\lambda^{\prime}\right)\right\|_{l_{k^{*}}^{2} \rightarrow L_{\lambda}^{2} l_{k^{\prime}}^{2}} ;$

(ii) $\left\|\widetilde{H}_{k k^{*}}(\lambda)\right\|_{L_{\lambda}^{2} l_{k}^{2} l_{k^{*}}^{2}} \lesssim\left\|\widetilde{\Theta}_{k, k^{\prime}}\left(\lambda, \lambda^{\prime}\right)\right\|_{L_{\lambda, \lambda^{\prime}}^{2} l_{k, k^{\prime}}^{2}}\left\|h_{k^{\prime} k^{*}}\left(\lambda^{\prime}\right)\right\|_{l_{k^{*}}^{2} \rightarrow L_{\lambda^{\prime}}^{2} l_{k^{\prime}}^{2}}$;

(iii) $\left\|\widetilde{H}_{k k^{*}}(\lambda)\right\|_{L_{\lambda}^{2} l_{k}^{2} l_{k^{*}}^{2}} \leq\left\|\widetilde{\Theta}_{k, k^{\prime}}\left(\lambda, \lambda^{\prime}\right)\right\|_{L_{\lambda^{\prime}}^{2} l_{k^{\prime}}^{2} \rightarrow L_{\lambda}^{2} l_{k}}\left\|h_{k^{\prime} k^{*}}\left(\lambda^{\prime}\right)\right\|_{L_{\lambda^{\prime}}^{2} l_{k^{\prime}, k^{*}}^{2}}$, 
where we mean

$$
\left\|h_{k^{\prime} k^{*}}\left(\lambda^{\prime}\right)\right\|_{l_{k^{*}}^{2} \rightarrow L_{\lambda^{\prime}}^{2} l_{k^{\prime}}^{2}}:=\left\|h\left(\lambda^{\prime}\right)\right\|_{L_{x}^{2} \rightarrow L_{\lambda^{\prime}}^{2} L_{x}^{2}} .
$$

Proof. (iii) is relatively simple. For fixed $k^{*}$, viewing $\widetilde{\Theta}_{k k^{\prime}}\left(\lambda, \lambda^{\prime}\right)$ as the kernel of the operator from $L_{\lambda^{\prime}}^{2} l_{k^{\prime}}^{2}$ to $L_{\lambda}^{2} l_{k}^{2}$, we have

$$
\left\|\widetilde{H}_{k k^{*}}(\lambda)\right\|_{L_{\lambda}^{2} l_{k}^{2}} \leq\left\|\widetilde{\Theta}_{k, k^{\prime}}\left(\lambda, \lambda^{\prime}\right)\right\|_{L_{\lambda^{\prime}}^{2} l_{k^{\prime}}^{2} \rightarrow L_{\lambda}^{2} l_{k}^{2}}\left\|h_{k^{\prime} k^{*}}\left(\lambda^{\prime}\right)\right\|_{L_{\lambda^{\prime}}^{2} l_{k^{\prime}}^{2}} .
$$

Taking $l_{k^{*}}^{2}$ to both sides and by Fubini, we obtain (iii).

To prove (i) and (ii), recall that $h_{k^{\prime} k^{*}}\left(\lambda^{\prime}\right)=\left(h\left(\lambda^{\prime}\right)\left(\mathrm{e}_{k^{*}}\right), \mathrm{e}_{k^{\prime}}\right)_{L_{x}^{2}}$. Denote by $h^{*}\left(\lambda^{\prime}\right)$ the adjoint of $h\left(\lambda^{\prime}\right)$. By linearity, we have

$$
\begin{aligned}
\widetilde{H}_{k k^{*}}(\lambda) & =\sum_{k^{\prime}} \int \widetilde{\Theta}_{k k^{\prime}}\left(\lambda, \lambda^{\prime}\right)\left(h\left(\lambda^{\prime}\right)\left(\mathrm{e}_{k^{*}}\right), \mathrm{e}_{k^{\prime}}\right)_{L_{x}^{2}} d \lambda^{\prime} \\
& =\int\left(\mathrm{e}_{k^{*}}, \sum_{k^{\prime}} \widetilde{\widetilde{\Theta}}_{k k^{\prime}}\left(\lambda, \lambda^{\prime}\right) h^{*}\left(\lambda^{\prime}\right)\left(\mathrm{e}_{k^{\prime}}\right)\right)_{L_{x}^{2}} d \lambda^{\prime} \\
& =\left(\mathrm{e}_{k^{*}}, \int h^{*}\left(\lambda^{\prime}\right)\left(\sum_{k^{\prime}} \overline{\widetilde{\Theta}}_{k k^{\prime}}\left(\lambda, \lambda^{\prime}\right) \mathrm{e}_{k^{\prime}}\right) d \lambda\right)_{L_{x}^{2}} .
\end{aligned}
$$

Here we omit the issue of the legality of changing the order of the integration and the summation, which can be justified by a standard density argument.

Denote by $F_{k}\left(\lambda, \lambda^{\prime}\right)=\sum_{k^{\prime}} \widetilde{\widetilde{\Theta}}_{k k^{\prime}}\left(\lambda, \lambda^{\prime}\right) \mathrm{e}_{k^{\prime}}$. For fixed $k, \lambda$, viewing $\int h^{*}\left(\lambda^{\prime}\right) F_{k}\left(\lambda, \lambda^{\prime}\right) d \lambda^{\prime}$ as a function in $L^{2}(\mathbb{T})$, we have

$$
\left\|\widetilde{H}_{k k^{*}}(\lambda)\right\|_{l_{k^{*}}^{2}}^{2}:=\sum_{k^{*}}\left|\left(\mathrm{e}_{k^{*}}, \int h^{*}\left(\lambda^{\prime}\right) F_{k}\left(\lambda, \lambda^{\prime}\right) d \lambda^{\prime}\right)_{L_{x}^{2}}\right|^{2}=\left\|\int h^{*}\left(\lambda^{\prime}\right) F_{k}\left(\lambda, \lambda^{\prime}\right) d \lambda^{\prime}\right\|_{L_{x}^{2}}^{2},
$$

thanks to Plancherel. Now viewing $h$ as a linear operator from $L_{x}^{2}$ to $L_{\lambda^{\prime}}^{2} L_{x}^{2}$ with kernel $h_{k^{\prime} k^{*}}\left(\lambda^{\prime}\right)$, hence $h^{*}$ is from $L_{\lambda^{\prime}}^{2} L_{x}^{2}$ to $L_{x}^{2}$ and

$$
h^{*}(G):=\int h^{*}\left(\lambda^{\prime}\right)\left(G\left(\lambda^{\prime}\right)\right) d \lambda^{\prime} .
$$

By viewing $F_{k}(\lambda, \cdot)$ as a function (for fixed $k, \lambda$ ) in $L_{\lambda^{\prime}}^{2} L_{x}^{2}$, we have

$$
\int h^{*}\left(\lambda^{\prime}\right) F_{k}\left(\lambda, \lambda^{\prime}\right) d \lambda^{\prime}=h^{*}\left(F_{k}(\lambda, \cdot)\right) \text {. }
$$

Therefore,

$$
\begin{aligned}
\left\|\widetilde{H}_{k k^{*}}(\lambda)\right\|_{l_{k^{*}}^{2}} & =\left\|h^{*}\left(F_{k}(\lambda, \cdot)\right)\right\|_{L_{x}^{2}} \leq\left\|h^{*}\right\|_{L_{\lambda^{\prime}}^{2} L_{x}^{2} \rightarrow L_{x}^{2}}\left\|F_{k}\left(\lambda, \lambda^{\prime}\right)\right\|_{L_{\lambda^{\prime}}^{2} L_{x}^{2}} \\
& =\|h\|_{L_{x}^{2} \rightarrow L_{\lambda^{\prime}}^{2} L_{x}^{2}} \cdot\left\|\sum_{k^{\prime}} \widetilde{\Theta}_{k k^{\prime}}\left(\lambda, \lambda^{\prime}\right) \mathrm{e}_{k^{\prime}}\right\|_{L_{\lambda^{\prime}}^{2} L_{x}^{2}} \\
& =\|h\|_{L_{x}^{2} \rightarrow L_{\lambda^{\prime}}^{2} L_{x}^{2}} \cdot\left\|\widetilde{\Theta}_{k k^{\prime}}\left(\lambda, \lambda^{\prime}\right)\right\|_{L_{\lambda^{\prime}}^{2} l_{k^{\prime}}^{2}}
\end{aligned}
$$

Taking $L_{\lambda}^{q}$ and $l_{k}^{\infty}$ or $L_{\lambda}^{2} l_{k}^{2}$ to both sides, we obtain (i) and (ii), with respectively. The proof of Lemma 2.15 is complete.

2.5. Probability tool-box. We denote by $\mathcal{B}_{\leq N}\left(\mathcal{B}_{>N}\right)$, the Borel $\sigma$ algebra generated by $\left\{g_{k}(\omega):|k| \leq N\right\}\left(\left\{g_{k}(\omega):|k|>N\right\}\right)$, and $\mathcal{B}_{N}$ be the Borel $\sigma$ algebra generated by $\left\{g_{k}(\omega): \frac{N}{2}<|k| \leq N\right\}$. For a $\sigma$ algebra $\mathcal{B}$, we use the notation $X \in \mathcal{B}$ to mean that $X$ is $\mathcal{B}$-measurable and $X \perp \mathcal{B}$ to mean that $X$ is independent of $\mathcal{B}$.

Let $(\Omega, \mathcal{F}, \mathbb{P})$ be a probability space, $(\mathcal{X}, \mu)$ be a measure space and $\mathcal{G} \subset \mathcal{F}$ be a sub $\sigma$ algebra. Let $X, Y$ be two random variables and let $f(x, \omega)$ be a random function with value 
on $L^{r}(\mathcal{X}, \mu)$. We recall the following classical inequalities for the conditional expectations:

(i) Hölder: $\quad \mathbb{E}[|X Y| \mid \mathcal{G}] \leq\left(\mathbb{E}\left[|X|^{p} \mid \mathcal{G}\right]\right)^{\frac{1}{p}} \cdot\left(\mathbb{E}\left[|Y|^{p^{\prime}} \mid \mathcal{G}\right]\right)^{\frac{1}{p^{\prime}}}$

(ii) Minkowski: If $p \geq r \geq 1, \mathbb{E}\left[\|f(x, \omega)\|_{L_{x}^{r}}^{p} \mid \mathcal{G}\right] \leq\left\|\left(\mathbb{E}\left[|f(x, \omega)|^{p} \mid \mathcal{G}\right]\right)^{\frac{1}{p}}\right\|_{L_{x}^{r}}$;

(iii) Chebyshev: For any $\lambda>0$ and $p \in(0, \infty), \quad \mathbb{P}[|X|>\lambda \mid \mathcal{G}] \leq \frac{1}{\lambda^{p}} \mathbb{E}\left[|X|^{p} \mid \mathcal{G}\right]$.

Lemma 2.16 (Conditional Wiener Chaos). Let $\left(g_{j}(\omega)\right)_{j \in E}$ be a inpendent, identically distributed complex Gaussians and $E$ is a finite index set. Let $\mathcal{C}$ be a $\sigma$-algebra independent of $\left(g_{j}(\omega)\right)_{j \in E}$. Assume that $\left(c_{k_{1}, k_{2}, \cdots, k_{m}}(\omega)\right)_{\left(k_{1}, \cdots, k_{n}\right) \in \mathbb{N}^{m}}$ is a sequence of $\mathcal{C}$-measurable random variables. Then for any finite subset $S \subset E^{m}$ and $p \geq 1$, we have

$$
\begin{aligned}
& \left(\mathbb{E}\left[\left|\sum_{\left(k_{1}, k_{2}, \cdots, k_{m}\right) \in S} c_{k_{1}, k_{2}, \cdots, k_{m}}(\omega) \prod_{j=1}^{m} g_{k_{j}}^{\iota_{j}}(\omega)\right|^{2 p} \mid \mathcal{C}\right]\right)^{\frac{1}{2 p}} \\
\leq & C_{0}(2 p-1)^{\frac{m}{2}}\left(\mathbb{E}\left[\left|\sum_{\left(k_{1}, k_{2}, \cdots, k_{m}\right) \in S} c_{k_{1}, k_{2}, \cdots, k_{m}}(\omega) \prod_{j=1}^{m} g_{k_{j}}^{\iota_{j}}(\omega)\right|^{2} \mid \mathcal{C}\right]\right)^{\frac{1}{2}},
\end{aligned}
$$

where $g_{k_{j}}^{\iota_{j}}(\omega)=g_{k_{j}}(\omega)$ or $\bar{g}_{k_{j}}(\omega)$ and the uniform constant $C_{0}$ is independent of the set $S$, the $\sigma$-algebra $\mathcal{C}$ and the number $m$ and $p \geq 1$.

Proof. Since the conditional expectation can be viewed as partial integration for the product probability space $\Omega=\Omega_{1} \times \Omega_{2}$, the conclusion follows from the usual Wiener chaos estimate.

Corollary 2.17. Assume that $1 \leq p_{1}, p_{2}, \cdots, p_{n}<\infty$ and $E \subset \mathbb{N}$ is a finite index set. Let $\left(g_{j}\right)_{j \in E}$ be a sequence of independent standard complex Gaussians. Let $\mathcal{C}$ be a $\sigma$-algebra independent of the $\sigma$-algebra generated by $\left(g_{j}\right)_{j \in E}$. Let $\left(c_{k_{1}^{*}, \cdots, k_{m}^{*}}\left(z_{1}, \cdots, z_{n} ; \omega\right)\right)_{\left(k_{1}^{*}, \cdots, k_{m}^{*}\right) \in E^{m}}$ be a sequence of $\mathcal{C}$-measurable random variables with values in $L^{p_{1}}\left(Z_{1}\right) \times \cdots L^{p_{n}}\left(Z_{n}\right)$. Consider the function

$$
F\left(z_{1}, \cdots, z_{n}, \omega\right):=\sum_{\left(k_{1}^{*}, \cdots, k_{m}^{*}\right) \in E^{m}} c_{k_{1}^{*}, \cdots, k_{m}^{*}}\left(z_{1}, \cdots, z_{n} ; \omega\right) \prod_{j=1}^{m} g_{k_{j}}^{\iota_{j}}(\omega) .
$$

Assume that there exists some constant $\Lambda_{0}>0$, such that

$$
\left\|\left(\mathbb{E}\left[|F|^{2} \mid \mathcal{C}\right]\right)^{\frac{1}{2}}\right\|_{L_{z_{1}}^{p_{1} \ldots L_{z}}} \leq \Lambda_{0}^{p_{n}}
$$

then for any $R>0$, outside an exceptional set of probability ${ }^{2}<C \mathrm{e}^{-c R^{\frac{2}{m}}}$, we have

$$
\|F\|_{L_{z_{1}}^{p_{1} \ldots L_{z_{n}}^{p_{n}}} \leq R \Lambda_{0}}
$$

Proof. Let $p \geq 2 \max \left\{p_{1}, \cdots, p_{n}\right\}$. By the conditional Chebyshev and the Minkowski inequalities,

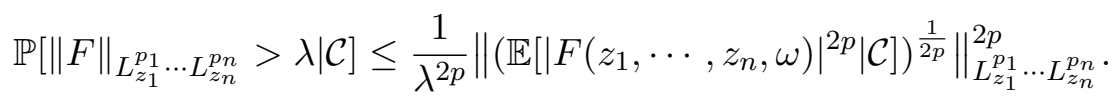

By Lemma 2.16 and the assumption (2.6), we have

$$
\left\|\left(\mathbb{E}\left[\left|F\left(z_{1}, \cdots, z_{n}, \omega\right)\right|^{2 p} \mid \mathcal{C}\right]\right)^{\frac{1}{2 p}}\right\|_{L_{z_{1}}^{p_{1}} \cdots L_{z_{n}}^{p_{n}}}^{2 p} \leq C^{2 p}(2 p)^{m p} \Lambda_{0}^{2 p}
$$

By choosing $\lambda=R \Lambda_{0}$ and optimizing the choice of $p$, we obtain that

$$
\mathbb{P}\left[\|F\|_{L_{z_{1}}^{p_{1} \ldots L_{z n}} p_{n}}>R \Lambda_{0} \mid \mathcal{C}\right] \leq C \mathrm{e}^{-c R^{\frac{2}{m}}} .
$$

By taking the expectation once to the inequality above, the proof of Corollary 2.17 is complete.

\footnotetext{
${ }^{2}$ This exceptional set depends on the random functions $c_{k_{1}^{*}, \cdots, k_{m}^{*}}$
} 


\section{Key iterative STEPS}

Though the smooth solutions $\left(v_{N}\right)_{N \in 2^{\mathbb{N}}}$ of $(1.7)$ with initial data $\Pi_{N} \phi^{\omega}$ already exist, the proof of Theorem 3 will be achieved by solving local-in-time fix-point problems. To this end, we recall the iteration scheme introduced in [17] with a slightly different setting, in order to solve the non-truncated equation.

3.1. Rigorous resolution scheme. Here we need to take into consideration of the timerestriction issue. Recall that $\chi \in C_{c}^{\infty}(\mathbb{R})$ is a bump function which is 1 on $\left[-\frac{1}{2}, \frac{1}{2}\right]$ and is zero outside $[-1,1]$. For $0<T<1$, we define $\chi_{T}(t):=\chi(t / T)$. We now rewrite the ansatz with time localization. We will use the notation $A^{\#}$ to define the quantities after time-localization procedure. In what follows, we describe inductive definition for the timerestriction that gives the rigorous resolution schem ${ }^{3}$. This contains four steps, including two fix-point problems:

-Initial step: We define

$$
w_{1}^{\#}(t):=w_{1}(t) \chi_{T}(t), \quad \psi_{\frac{1}{2}}^{M, \#}(t):=\chi(t) S_{\alpha}(t) \mathbf{P}_{M} \phi^{\omega}, \quad H_{k k^{*}}^{M, \frac{1}{2}, \#}=\chi(t) \mathrm{e}^{i t|k|^{\alpha}} \mathbf{1}_{k=k^{*}},
$$

for any dyadic number $M \geq \frac{1}{2}$.

-Induction assumption: Suppose that $M \geq 1$ is a given dyadic number and we have defined:

$$
w_{N}^{\#}(t), \forall N \leq M ; \quad h^{N^{\prime}, L, \#}, \forall L \leq L_{N^{\prime}}, L<M .
$$

We need to define $w_{2 M}^{\#}$ and $h^{N^{\prime}, M, \#}$ for all $N^{\prime}>M^{\frac{1}{1-\delta}}$ (i.e. $M \leq L_{N^{\prime}}$ ). First, note that for all $L \leq M, R<L^{1-\delta}<M, \zeta_{R}^{L, \#}:=\psi_{R}^{L, \#}-\psi_{\frac{R}{2}}^{L, \#}$ are well-defined, since they can be

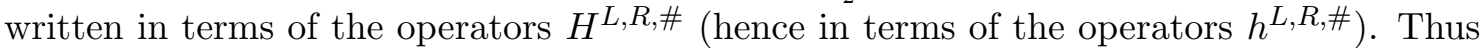
for all $L \leq N$, the following functions

$$
v_{L}^{\#}:=\sum_{\frac{1}{2} \leq L^{\prime} \leq L} y_{L^{\prime}}, \quad \text { where } y_{L^{\prime}}^{\#}:=\chi(t) S_{\alpha}(t) \mathbf{P}_{L^{\prime}} \phi^{\omega}+\sum_{\left(L^{\prime}, R^{\prime}\right): R^{\prime}<L^{\prime 1-\delta}} \zeta_{R^{\prime}}^{L^{\prime}, \#}+w_{L^{\prime}}(t)
$$

are also well-defined.

-Uniform bounds 1: Estimates for the linear operator: Next for $L=M$ and $N^{\prime}>M^{\frac{1}{1-\delta}}$, we define $H_{k k *}^{N^{\prime}, M \text {,\# }}$ by taking the $k$-th Fourier mode of the solution $\varphi^{\#}$ to the equation

$$
\varphi^{\#}(t)=\chi(t) S_{\alpha}(t)\left(\mathrm{e}_{k^{*}}\right)+2 i \chi_{T}(t) \cdot \Pi_{N^{\prime}} \mathcal{I} \mathcal{N}_{3}\left(\varphi^{\#}, \Pi_{M} v_{M}^{\#}, \Pi_{M} v_{M}^{\#}\right), \quad \text { where } \frac{N^{\prime}}{2}<\left|k^{*}\right| \leq N^{\prime} .
$$

We note that knowing $v_{M}$, for fixed $N^{\prime}$, the solution $\varphi^{\#}(t)$ exists and is unique, from a simple Grownwall type argument ${ }^{4}$. Since it is a linear equation, this will turn out to be true, if $T>0$ is sufficiently small.

-Uniform bounds 2: Estimates for the smooth remainder: We finally write down the equation of $w_{2 M}^{\#}$ to finish the induction step. Since $L_{2 M}<(2 M)^{1-\delta} \leq M$ (true for large $M$, the case that we concern), the function $\psi_{L_{2 M}}^{2 M, \#}:=\mathcal{H}^{2 M, L_{2 M}, \#}\left(\mathbf{P}_{2 M} \phi^{\omega}\right)$ is well-defined. Now we define $w_{2 M}^{\#}$ by solving the following equation:

$$
\begin{aligned}
w_{2 M}^{\#}(t)= & -i \chi_{T}(t) \mathcal{I}\left[\mathcal{N}\left(w_{2 M}^{\#}+\psi_{L_{2 M}}^{2 M, \#}+v_{M}^{\#}\right)-\mathcal{N}\left(v_{M}^{\#}\right)\right] \\
& -2 i \chi_{T}(t) \Pi_{2 M} \mathcal{I} \mathcal{N}_{3}\left(\psi_{L_{2 M}}^{2 M, \#}, \Pi_{L_{2 M}} v_{L_{2 M}}^{\#}, \Pi_{L_{2 M}} v_{L_{2 M}}^{\#}\right) .
\end{aligned}
$$

Note that to solve $w_{2 M}^{\#}$ through (3.2), we expand the right side of (3.2), the resulting terms can be grouped as follows:

\footnotetext{
${ }^{3}$ Since for the truncated initial data, the global smooth solution exists and is unique, the fix-point procedure used here is only to establish required bounds in suitable function spaces.

${ }^{4}$ More precisely, we may multiply both sides by $\overline{\varphi^{\#}}$ and doing the integration by part. This allows us to control $\frac{d}{d t}\left\|\varphi^{\#}(t)\right\|_{L^{2}(\mathbb{T})}^{2}$ by $C\left(N^{\prime}, v_{M}\right)\left\|\varphi^{\#}(t)\right\|_{L^{2}(\mathbb{T})}^{2}$.
} 
1) At least two entries in $\mathcal{I N}_{3}(\cdot, \cdot, \cdot)$ are $\psi_{L_{2 M}}^{2 M \text {,\# }}$ and the other one (if not the same) is $v_{M}^{\#}$;

2) Exactly one entry in $\mathcal{I N}_{3}(\cdot, \cdot, \cdot)$ is $\psi_{L_{2 M}}^{2 M}$, at least one $v_{M}^{\#}-\Pi_{L_{2 M}} v_{L_{2 M}}^{\#}$, and the last one (if it is different from the two) is $v_{M}^{\#}$;

3) $\mathcal{N}_{3}\left(v_{M}^{\#}, \psi_{L_{2 M}}^{2 M, \#}, v_{M}^{\#}\right)$;

4) At least two $w_{2 M}^{\#}$ or exactly one $w_{2 M}^{\#}$ and at least one $\psi_{L_{2 M}}^{2 M}$.

5) Exactly one $w_{2 M}^{\#}$ and all others equal $v_{M}^{\#}$;

6) The projective term: $\Pi_{2 M}^{\perp} \mathcal{I} \mathcal{N}_{3}\left(\psi_{L_{2 M}}^{2 M, \#}, v_{M}^{\#}, v_{M}^{\#}\right)$;

7) The diagonal nonlinear term $\mathcal{I R}_{0}:=\mathcal{I N}_{0}\left(w_{2 M}^{\#}+\psi_{L_{2 M}}^{2 M, \#}+v_{M}^{\#}\right)-\mathcal{I N}_{0}\left(v_{M}^{\#}\right)$.

3.2. Key multi-linear terms. First we describe the key multi-linear terms in order to estimate the linear operators through (3.1), Since the solution $\varphi^{\#}$ of $(3.1)$ is $\mathcal{H}^{N^{\prime}, M, \#}\left(\mathrm{e}_{k^{*}}\right)$, by taking the difference $\mathcal{H}^{N^{\prime}, M, \#}\left(\mathrm{e}_{k^{*}}\right)-\mathcal{H}^{N^{\prime}, \frac{M}{2}}$,\# $\left(\mathrm{e}_{k^{*}}\right)$, we have

$$
h^{N^{\prime}, M, \#}\left(\mathrm{e}_{k^{*}}\right)=2 i \chi_{T}(t) \mathcal{I} \Pi_{N^{\prime}} \mathcal{N}_{3}\left(h^{N^{\prime}, M, \#}\left(\mathrm{e}_{k^{*}}\right), \Pi_{M} v_{M}^{\#}, \Pi_{M} v_{M}^{\#}\right)+2 i \mathcal{P}_{N^{\prime}, M}^{+}\left(H^{N^{\prime}, \frac{M}{2}}\left(\mathrm{e}_{k^{*}}\right)\right) \text {, }
$$

where the operator $\mathcal{P}_{N^{\prime}, L}^{+}$is defined by

$$
\mathcal{P}_{N^{\prime}, L}^{+}(w):=\chi_{T}(t) \cdot \mathcal{I} \Pi_{N^{\prime}}\left[\mathcal{N}_{3}\left(w, \Pi_{L} v_{L}^{\#}, \Pi_{L} v_{L}^{\#}\right)-\mathcal{N}_{3}\left(w, \Pi_{\frac{L}{2}} v_{\frac{L}{2}}^{\#}, \Pi_{\frac{L}{2}} v_{\frac{L}{2}}^{\#}\right)\right]
$$

for $L>\frac{1}{2}$ and

$$
\mathcal{P}_{N^{\prime}, \frac{1}{2}}^{+}(w):=\chi_{T}(t) \cdot \mathcal{I} \Pi_{N^{\prime}} \mathcal{N}_{3}\left(w, \Pi_{\frac{1}{2}} v_{\frac{1}{2}}^{\#}, \Pi_{\frac{1}{2}} v_{\frac{1}{2}}^{\#}\right) .
$$

We will need an analogue of the operator above later:

$$
\mathcal{P}_{N^{\prime}, L}^{-}(w):=\chi_{T}(t) \cdot \mathcal{I}\left[\Pi_{M^{\prime}} \mathcal{N}_{3}\left(\Pi_{L} v_{L}^{\#}, w, \Pi_{L} v_{L}^{\#}\right)-\Pi_{N^{\prime}} \mathcal{N}_{3}\left(\Pi_{\frac{L}{2}} v_{\frac{L}{2}}^{\#}, w, \Pi_{\frac{L}{2}} v_{\frac{L}{2}}^{\#}\right)\right]
$$

for $L>\frac{1}{2}$ and

$$
\mathcal{P}_{N^{\prime}, \frac{1}{2}}^{-}(w):=\chi_{T}(t) \cdot \mathcal{I} \Pi_{N^{\prime}} \mathcal{N}_{3}\left(\Pi_{\frac{1}{2}} v_{\frac{1}{2}}^{\#}, w, \Pi_{\frac{1}{2}} v_{\frac{1}{2}}^{\#}\right) .
$$

Now (3.1) is reduced to the following equation:

$$
\begin{aligned}
h^{N^{\prime}, M, \#}\left(\mathrm{e}_{k^{*}}\right) & =2 i \sum_{L \leq M} \mathcal{P}_{N^{\prime}, L}^{+}\left(h^{N^{\prime}, M, \#}\left(\mathrm{e}_{k^{*}}\right)\right)+2 i \sum_{L<M} \mathcal{P}_{N^{\prime}, M}^{+}\left(h^{N^{\prime}, L, \#}\left(\mathrm{e}_{k^{*}}\right)\right) \\
& +2 i \mathcal{P}_{N^{\prime}, M}^{+}\left(H^{N^{\prime}, \frac{1}{2}, \#}\left(\mathrm{e}_{k^{*}}\right)\right) .
\end{aligned}
$$

To deal with (3.2), we need to treat the terms of type 1)-7). By definition, we have the decompositions

$$
\begin{gathered}
v_{M}^{\#}=\sum_{N \leq M}\left(\psi_{L_{N}}^{N, \#}+w_{N}^{\#}\right), \quad v_{M}^{\#}-\Pi_{L_{2 M}} v_{L_{2 M}}^{\#}=\sum_{L_{2 M}<R \leq M}\left(\psi_{L_{R}}^{R, \#}+w_{R}^{\#}\right)+\Pi_{L_{2 M}}^{\perp} v_{L_{2 M}}^{\#}, \\
\psi_{L_{N}}^{N, \#}=\chi(t) S_{\alpha}(t) \mathbf{P}_{N} \phi^{\omega}+\sum_{L \leq L_{N}} \zeta_{L}^{N, \#} .
\end{gathered}
$$

We now precise the multi-linear terms according to their types. To simplify the notation, we will not write $(\cdot)^{\#}$ for the time-restriction here, and we mean $\zeta^{2 M}$ by a term of the form $\zeta_{L}^{2 M}$ for some $L \leq L_{2 M}$ or $\psi_{\frac{1}{2}}^{2 M}$.

1) $\mathcal{I N}_{3}\left(\zeta^{2 M}, \zeta^{2 M}, *\right), \quad \mathcal{I} \mathcal{N}_{3}\left(\zeta^{2 M}, *, \zeta^{2 M}\right)$;

2) $\mathcal{I N}_{3}\left(\zeta^{2 M}, w_{N}+\psi_{L_{N}}^{N}+\Pi_{L_{2 M}}^{\perp} v_{L_{2 M}}^{\#}, *\right), \quad \mathcal{I N}_{3}\left(\zeta^{2 M}, *, w_{N}+\psi_{L_{N}}^{N}+\Pi_{L_{2 M}}^{\perp} v_{L_{2 M}}^{\#}\right)$, $\mathcal{I N}_{3}\left(w_{M}+\psi_{L_{N}}^{N}+\Pi_{L_{2 M}}^{\perp} v_{L_{2 M}}^{\#}, \zeta^{2 M}, *\right)$ with some $N \geq M^{1-\delta}$

3) $\mathcal{I N}_{3}\left(*, \zeta^{2 N}, *\right)$; 
4) $\mathcal{I N}_{3}\left(w_{2 M}\right), \quad \mathcal{I N}_{3}\left(w_{2 M}, *, w_{2 M}\right), \quad \mathcal{I N}_{3}\left(w_{2 M}, w_{2 M}, *\right)$, $\mathcal{I N}_{3}\left(w_{M}, \zeta^{2 M}, *\right), \quad \mathcal{I N}_{3}\left(\zeta^{2 M}, w_{2 M}, *\right), \quad \mathcal{I N}_{3}\left(\zeta^{2 M}, *, w_{2 M}\right)$

5) $\mathcal{I N}_{3}\left(w_{2 M}, v_{M}, v_{M}\right), \quad \mathcal{I N}_{3}\left(v_{M}, w_{2 M}, v_{M}\right)$

6) $\Pi_{2 M}^{\perp} \mathcal{I} \mathcal{N}_{3}\left(\zeta^{2 M}, v_{M}, v_{M}\right)$

7) Trivial resonances: $\mathcal{I R}_{0}$.

where the input $*$ stands for a term belonging to the set of functions

$$
\left\{v_{M}, \Pi_{L_{2 M}} v_{L_{2 M}}, \zeta^{2 M}\right\} .
$$

In summary, the only possible high-low-low interactions appear in the following situations:

- Case 3$)$, but $\zeta^{2 M}$ is in the "good" position.

- Case 5), but with $w_{2 M}$ who has the dominated frequency. These terms can be viewed as errors of certain linearization procedure and will be treated by the operator $\mathcal{P}^{ \pm}$defined later.

- Pseudo high-low interactions 5 . Terms in Case 2) and Case 5) involving the entry $\Pi_{L_{2 M}}^{\perp} v_{L_{2 M}}$ or $\Pi_{M}^{\perp} v_{M}$. Though these are not high-low interaction, when we decompose $v_{M}=\sum_{M^{\prime} \leq M} y_{M^{\prime}}$, the portions $y_{M^{\prime}}$ coming from $M^{\prime} \ll M$ may not have sufficient decay in the estimates, they behave like just $y_{M^{\prime}}$ in a priori. This is caused by the fact that the Fourier support of $v_{M}$ is not bounded, when we truncate only the initial data. Extra estimates for $\Pi_{M}^{\perp} v_{M}$ is needed.

3.3. Induction step. Now we summarize the induction step. First we define the linear operators (with $L<N^{1-\delta}$ ):

$$
\mathcal{P}_{N, L}^{+}(w):=\chi_{T}(t) \cdot \mathcal{I} \Pi_{N}\left[\mathcal{N}_{3}\left(w, \Pi_{L} v_{L}^{\#}, \Pi_{L} v_{L}^{\#}\right)-\mathcal{N}_{3}\left(w, \Pi_{\frac{L}{2}} v_{\frac{L}{2}}^{\#}, \Pi_{\frac{L}{2}} v_{\frac{L}{2}}^{\#}\right)\right]
$$

and

$$
\mathcal{P}_{N, L}^{-}(w):=\chi_{T}(t) \cdot \mathcal{I} \Pi_{N}\left[\mathcal{N}_{3}\left(\Pi_{L} v_{L}^{\#}, w, \Pi_{L} v_{L}^{\#}\right)-\mathcal{N}_{3}\left(\Pi_{\frac{L}{2}} v_{\frac{L}{2}}^{\#}, w, \Pi_{\frac{L}{2}} v_{\frac{L}{2}}^{\#}\right)\right]
$$

for $L \leq L_{N}=\max \left\{L^{\prime}: L^{\prime}<N^{1-\delta}\right\}$. Denote by $\Theta_{k, k^{\prime}}^{N, L}\left(t, t^{\prime}\right)$ the kernel of the operator $\mathcal{P}_{N, L}^{+}$. Note that on the support of $\Theta_{k k^{\prime}}^{N, L}\left(t, t^{\prime}\right),\left|k-k^{\prime}\right| \lesssim L$. Following [17], for a given dyadic number $M$, we call $\operatorname{Loc}(M)$ the following uniform bounds: for all $(L, N)$, such that $\frac{1}{2}<L<M, L<N^{1-\delta}$,

(i) $\left\|h^{N, L, \#}\right\|_{Y^{b}} \leq L^{-\delta_{0}^{2}},\left\|h^{N, L, \#}\right\|_{S^{b, q}} \leq N^{\epsilon_{1}} L^{-\nu},\left\|h^{N, L, \#}\right\|_{Z^{b}} \leq N^{1-\frac{\alpha}{2}+\epsilon_{1}} L^{-\nu}$;

(ii) $\left\|\zeta_{L}^{N, \#}\right\|_{\substack{0, \frac{2 b_{0}}{X_{\infty, q} q^{\prime}} \\ \text { (ij) }}} \leq N^{\epsilon_{2}} L^{-\nu},\left\|\zeta_{L}^{N, \#}\right\|_{X^{0, b_{0}}} \leq N^{-(\alpha-1)+\epsilon_{2}} L^{-\nu}$

(iii) $\left\|\zeta_{L}^{N, \#}\right\|_{L_{t}^{4} L_{x}^{\infty}} \leq N^{-(\alpha-1)+\epsilon_{2}} L^{\frac{1}{2}-\nu}$

(iv) $\left\|\left\langle\frac{\left|k-k^{*}\right|}{L}\right\rangle^{\kappa} h_{k k^{*}}^{N, L, \#}\right\|_{Z^{b}} \leq N$;

(v) $\left\|\mathcal{P}_{N, L}^{ \pm}\right\|_{X^{0, b} \rightarrow X^{0, b}} \leq T^{\theta} L^{-\delta_{0}}$;

(vi) $\quad\left\|\mathbf{1}_{|k|,\left|k^{\prime}\right| \geq \frac{N}{4}} \cdot \Theta_{k k^{\prime}}^{N, L}\left(t, t^{\prime}\right)\right\|_{Z^{b, b}} \leq T^{\theta} N^{1-\frac{\alpha}{2}+\epsilon_{1}} L^{-\nu}$;

(vii) $\quad\left\|\mathbf{1}_{|k|,\left|k^{\prime}\right| \geq \frac{N}{4}} \cdot \Theta_{k k^{\prime}}^{N, L}\left(t, t^{\prime}\right)\right\|_{S^{b, b}} \leq T^{\theta} N^{\epsilon_{1}} L^{-\nu}$;

(viii) $\left\|w_{N}^{\#}\right\|_{X^{0, b}} \leq N^{-s},\left\|\Pi_{N_{0}}^{\perp} w_{N}^{\#}\right\|_{X^{0, b}} \leq N_{0}^{-s} \quad \forall N \leq M, N_{0} \geq N$.

\footnotetext{
${ }^{5}$ This is the main different issue compared to the truncated FNLS.
} 
Remark 3.1. Hierarchy of numerical constants: Let $\sigma>0$ be the free small parameter to choose.

$$
\begin{aligned}
& b_{0}=0.5+\sigma^{200}, b=0.5+2 \sigma^{200}, b_{1}=0.5+3 \sigma^{200}, \theta=0.01 \sigma^{200}, q^{-1}=\sigma^{50}, \kappa=\sigma^{-500} \\
& \epsilon_{1}=\sigma^{2}, \epsilon_{2}=\epsilon_{1}+100 \sigma^{5}, \delta=\sigma^{20}, \delta_{0}=\sigma^{10}, s=\frac{1}{2}-\frac{\alpha}{4}+\sigma ; \\
& \nu=\min \left\{\frac{1}{2}-\frac{\alpha}{4}, \frac{7(\alpha-1)}{4}\right\}-\sigma .
\end{aligned}
$$

With these choices, for $\sigma>0$ small enough, we have

$$
\begin{aligned}
& \nu \leq \min \left\{s, \frac{7(\alpha-1)}{4}\right\}-100\left(\epsilon_{1}+\epsilon_{2}\right), \frac{b_{1}-\frac{1}{2}}{q^{\prime}-1} \ll q^{\prime}-2 b_{1} \ll 1 ; \\
& \epsilon_{1}>100\left(b_{1}-\frac{1}{2}+\frac{1}{q}+\theta\right), \quad(\alpha-1)+2 s \nu-s>0 .
\end{aligned}
$$

In the remaining part of this article, all these numerical constants are reserved to depend only on the free small parameter $\sigma$, which will be chosen small enough if necessary.

Remark 3.2. In the induction argument, the condition (i) for the next step is inherent from (v), the condition (ii) is inherent from (vii), and the condition (iii) is inherent from (vi). The condition (iv) means that the support of $h_{k, k^{*}}^{N, L}$ is essentially restricted on $\left|k-k^{*}\right| \lesssim L$.

The key inductive proposition is the following:

Proposition 3.3. Assume that $\alpha \in\left(\alpha_{0}, \frac{6}{5}\right]$. There exists $\sigma>0$, sufficiently small and we fix the numerical constants as in Remark 3.1. Suppose that Loc(M) is true for all $\omega \in \Omega^{*}$. Then there exists a measurable set $\Omega_{1}^{*} \subset \Omega^{*}$ with the property that (for some $\theta>0) \mathbb{P}\left[\Omega^{*} \backslash \Omega_{1}^{*}\right]<C_{\theta} \mathrm{e}^{-T^{-\theta} M^{\theta}}$, such that for all $\omega \in \Omega_{1}^{*}$, the statement Loc $(2 M)$ is true. Consequently, outside a exceptional set of probability $<C_{\theta} \mathrm{e}^{-T^{-\theta}}$, the statement Loc(M) holds true for every dyadic number $M \geq \frac{1}{2}$.

Remark 3.4. The main reason for the constraint $\alpha>\alpha_{0}$ is the condition

$$
(\alpha-1)+2 s \nu-s>0 .
$$

By numerical computation, one verifies easily that, for sufficiently small choice of the free parameter $\sigma>0$, the above condition holds if $\alpha>\alpha_{0}$.

Using Proposition 3.3, we can easily deduce Theorem 3, (i) and (ii). Indeed, we first delete a set of probability smaller than $C_{\theta} \mathrm{e}^{-T^{-\theta}}$ such that the statements $\operatorname{Loc}(M)$ are true for all dyadic $M \geq \frac{1}{2}$ on the interval $[-T, T]^{6}$. In particular, for each dyadic number $M$, we have

$$
v_{M}^{\#}=\sum_{N \leq M}\left(\chi(t) S_{\alpha}(t) \mathbf{P}_{N} \phi^{\omega}+w_{N}^{\#}\right)+\sum_{N \leq M} \sum_{\frac{1}{2}<L \leq L_{N}} \zeta_{L}^{N, \#},
$$

satisfying the estimates (i) to (viii) listed in the hypothesis $\operatorname{Loc}(M)$. Therefore, $w^{\#}=$ $\sum_{M} w_{M}^{\#}$ is a convergent sequence in $X_{T}^{\frac{1}{2}-\frac{\alpha}{4}+\epsilon, \frac{1}{2}+\epsilon}$, and $\zeta^{\#}=\sum_{N, L} \zeta_{L}^{N, \#}$ is a convergent sequence in $L^{\infty}\left([-T, T] ; H^{\alpha-1-\epsilon}(\mathbb{T})\right) \cap L^{\infty}\left([-T, T] ; \mathcal{F} L^{\frac{\alpha}{2}-\epsilon, \infty}(\mathbb{T})\right)$. Now for fixed $N$, the smooth solution $v_{M}$ and $v_{M}^{\#}$ are both solutions of 1.7 with the same initial data $\Pi_{N} \phi^{\omega}$. By uniquenss of the smooth solution, when restricting to a smaller time interval, say $[-T / 2, T / 2]$, we have $v_{M}^{\#}=v_{M}$. This allows us to decompose $v_{N}$ similarly as sums of $S(t) \Pi_{N} \phi^{\omega}, \zeta_{L}^{N}$ and $w_{N}$. Moreover, the same equations (3.1) and 3.2 hold for if we drop the \# notation and the time truncation $\chi_{T}(t)$. This shows that $\zeta_{L}^{N}, w_{N}$ coincide with $\zeta_{L}^{N, \#}, w_{N}^{\#}$ on $[-T / 2, T / 2]$. This proves Theorem 3 , (i) and (ii).

\footnotetext{
${ }^{6}$ Note that $T$ is involved in the time cutoff functions to define $\zeta_{L}^{N, \#}, h^{N, L, \#}, w_{N}^{\#}$.
} 
3.4. The key multilinear estimate. We make the following assumptions on $\widetilde{v}_{j}\left(\lambda_{j}, k_{j}\right)$ : -Type (G) Gaussian:

$$
\widetilde{v}_{j}\left(\lambda_{j}, k_{j}\right)=\widetilde{\psi}_{\frac{1}{2}}^{N_{j}}\left(\lambda_{j}, k_{j}\right):=\mathbf{1}_{\frac{N_{j}}{2}<\left|k_{j}\right| \leq N_{j}} \frac{g_{k_{j}}(\omega)}{\left[k_{j}\right]^{\frac{\alpha}{2}}} \widehat{\chi}\left(\lambda_{j}\right)
$$

with the bounds

$$
\left\|v_{j}\right\|_{X^{0, b}} \leq N_{j}^{-\frac{\alpha-1}{2}+\epsilon_{1}},\left\|v_{j}\right\|_{\substack{0, \frac{2 b}{q^{\prime}} \\ q_{\infty}, q}} \leq N_{j}^{-\frac{\alpha}{2}+\epsilon_{1}}
$$

-Type (C) Colored:

$$
\widetilde{v}_{j}\left(\lambda_{j}, k_{j}\right)=\widetilde{\zeta}_{L_{j}}^{N_{j}}\left(\lambda_{j}, k_{j}\right)=\sum_{\frac{N_{j}}{2}<\left|k_{j}^{*}\right| \leq N_{j}} \widetilde{h}_{k_{j} k_{j}^{*}}^{N_{j}, L_{j}}\left(\lambda_{j}\right) \frac{g_{k_{j}^{*}}(\omega)}{\left[k_{j}^{*}\right]^{\frac{\alpha}{2}}},
$$

where $1 \leq L_{j} \leq L_{N_{j}}<N_{j}^{1-\delta}$

$$
\operatorname{supp}\left(\widetilde{h}_{k_{j} k_{j}^{*}}^{N_{j}, L_{j}}\right) \subset\left\{\left|k_{j}\right| \leq N_{j}, \frac{N_{j}}{2}<\left|k_{j}^{*}\right| \leq N_{j}\right\}
$$

and $\widetilde{h}_{k_{j} k_{j}^{*}}^{N_{j}, L_{j}}$ is $\mathcal{B}_{\leq L_{j}}$ measurable. Moreover, we assume that

$$
\begin{aligned}
& Y^{b} \text { norm: }\left\|\left\langle\lambda_{j}\right\rangle^{b} \widetilde{h}_{k_{j} k_{j}^{*}}^{N_{j} L_{j}}\left(\lambda_{j}\right)\right\|_{l_{k_{j}^{*}}^{2} \rightarrow l_{k_{j}}^{2} L_{\lambda_{j}}^{2}} \leq L_{j}^{-\delta_{0}^{2}}, \\
& S^{b, q} \text { norm: }\left\|\left\langle\lambda_{j}\right\rangle^{\frac{2 b}{q^{\prime}}} \widetilde{h}_{k_{j} k_{j}^{*}}^{N_{j}, L_{j}}\left(\lambda_{j}\right)\right\|_{l_{k_{j}}^{\infty} L_{\lambda_{j}}^{q} l_{k_{j}^{*}}^{2}} \leq N_{j}^{\epsilon_{1}} L_{j}^{-\nu} \\
& Z^{b} \text { norm: }\left\|\left\langle\lambda_{j}\right\rangle \widetilde{h}_{k_{j} k_{j}^{*}}^{N_{j}, L_{j}}\left(\lambda_{j}\right)\right\|_{L_{\lambda_{j}}^{2} l_{k_{j}}^{2} l_{k_{j}^{*}}^{2}} \leq N_{j}^{1-\frac{\alpha}{2}+\epsilon_{1}} L_{j}^{-\nu} \\
& L^{\infty} \operatorname{norm}:\left\|v_{j}\right\|_{L_{t}^{4} L_{x}^{\infty}} \leq N_{j}^{-(\alpha-1)+\epsilon_{2}} L_{j}^{\frac{1}{2}-\nu} \\
& X^{0, b_{0}} \text { norm: }\left\|v_{j}\right\|_{X^{0, b_{0}}} \leq N_{j}^{-(\alpha-1)+\epsilon_{2}} L_{j}^{-\nu} \\
& X_{\infty, q}^{0, \frac{2 b_{0}}{q^{\prime}}} \text { norm: }\left\|v_{j}\right\|_{X_{\infty, q}^{0, \frac{2 b_{0}}{q^{\prime}}}} \leq N_{j}^{-\alpha+\epsilon_{2}} L_{j}^{-\nu}
\end{aligned}
$$

and the almost localization condition:

$$
\left\|\left\langle\lambda_{j}\right\rangle^{b}\left\langle\frac{\left|k_{j}-k_{j}^{*}\right|}{L_{j}}\right\rangle^{\kappa} \widetilde{h}_{k_{j} k_{j}^{*}}^{N_{j}, L_{j}}\left(\lambda_{j}\right)\right\|_{L_{\lambda_{j} k_{j} k_{j}^{*}}^{2} l^{2}} \leq N_{j} .
$$

-Type (D) Deterministic:

$$
\left\|\left\langle\lambda_{j}\right\rangle^{b} \widetilde{v}_{j}\left(\lambda_{j}, k_{j}\right)\right\|_{l_{k_{j}}^{2} L_{\lambda_{j}}^{2}} \leq N_{j}^{-s},\left\|\left\langle\lambda_{j}\right\rangle^{b} \widetilde{v}_{j}\left(\lambda_{j}, k_{j}\right) \mathbf{1}_{\left|k_{j}\right|>N_{0}}\right\|_{l_{k_{j}}^{2} L_{\lambda_{j}}^{2}} \leq N_{0}^{-s}, \forall N_{0} \geq N_{j} .
$$

For functions $v_{j}$ of the form $(\mathrm{G}),(\mathrm{C})$ or $(\mathrm{D})$, they are all associated with dyadic numbers $N_{j}$ (or $\left(N_{j}, L_{j}\right)$ for the type $(\mathrm{C})$ ). In order to organize the terms in a unified way, we will call that $\left(N_{j}, L_{j}\right)$ a characterized pair for a function $v_{j}$ of the form $(\mathrm{G}),(\mathrm{C})$ or $(\mathrm{D})$, where we use the convention $L_{j}=\frac{1}{2}$ if $v_{j}$ is of type (G) while $L_{j}=2 L_{N_{j}}$ if $v_{j}$ is of type (D). We will also call $N_{j}$ the characterized frequency of $v_{j}$, in the sense that the Fourier support of $v_{j}$ is essentially localized at scale $N_{j}$. Recall that $N_{(1)} \geq N_{(2)} \geq \cdots$ is the non-increasing rearrangement of $N_{1}, N_{2}, \cdots$, we will denote by $v_{(j)}$, the function in the set $\left\{v_{1}, v_{2}, \cdots\right\}$ with the characterized frequency $N_{(j)}$. Yet, the order of $L_{(1)}, L_{(2)}, \cdots$ is not specified.

The proof of Proposition 3.3 consists of solving the fix-point problem 1 and the fixpoint problem 2. The following two propositions are crucial for solving the first fix-point problem. Recall that $\Theta_{k, k_{1}}^{N, L}\left(t, t^{\prime}\right)$ is the kernel of the operator $\mathcal{P}_{N, L}^{+}$defined in (3.6). 
Proposition 3.5. Assume that $\Omega_{0} \subset \Omega$ is such that (3.9) $-(3.17)$ holds for all $N_{j} \leq L$. Then outside a set of probability $<\mathrm{e}^{-c L^{\theta} R}$ and any $N>L^{\frac{1}{1-\delta}}$, we have

$$
\left\|\Theta_{k, k_{1}}^{N, L}\left(t, t_{1}\right) \mathbf{1}_{|k|,\left|k_{1}\right| \geq \frac{N}{4}}\right\|_{S^{b_{1}, b, q}} \leq R N^{100\left(\frac{1}{q}+\kappa^{-0.1}+b_{1}-\frac{1}{2}\right)} L^{-\nu},
$$

provided that $\nu \leq \min \left\{s, \frac{7(\alpha-1)}{4}\right\}-10\left(\epsilon_{1}+\epsilon_{2}\right)$.

Proposition 3.6. Assume that $\Omega_{0} \subset \Omega$ is such that (3.9) -(3.17) holds for all $N_{j} \leq L$. Then outside a set of probability $<\mathrm{e}^{-c L^{\theta} R}$ and any $N>L^{\frac{1}{1-\delta}}$, we have

$$
\left\|\Theta_{k, k_{1}}^{N, L}\left(t, t_{1}\right) \mathbf{1}_{|k|,\left|k_{1}\right| \geq \frac{N}{4}}\right\|_{Z^{b_{1}, b}} \leq R N^{100\left(\frac{1}{q}+\kappa^{-0.1}+b_{1}-\frac{1}{2}\right)} L^{-\nu},
$$

provided that $\nu \leq \min \left\{s, \frac{7(\alpha-1)}{4}\right\}-10\left(\epsilon_{1}+\epsilon_{2}\right)$.

The following proposition is crucial to solve the fix-point problem 2:

Proposition 3.7. Assume that $\alpha>\alpha_{0}$. There exists $\sigma>0$, sufficiently small in the definition of the numerical constants in Remark 3.1. such that the following holds true: Suppose that $v_{1}, v_{2}, v_{3}$ are of type $(G),(C)$ or $(D)$ with characterized parameters $\left(N_{j}, L_{j}\right)$, $j=1,2,3$, with respectively. Then outside an exceptional set of probability $<\mathrm{e}^{-N_{(1)}^{\theta} R^{\frac{2}{3}}}$, independent of the choice of functions $v_{j}$ of type $(D)$, we have

(1) If $v_{(1)}$ is of type $(G)$ or $(C)$ and $N_{(2)} \gtrsim N_{(1)}^{1-\delta}$, we have

$$
\left\|\mathcal{I} \mathcal{N}_{3}\left(v_{1}, v_{2} \cdot v_{3}\right)\right\|_{X^{0, b_{1}}} \lesssim R N_{(1)}^{-s} N_{(2)}^{-\delta_{0}} .
$$

(2) If $N_{2} \gg N_{1}, N_{3}$ and $v_{2}$ is of type $(G)$ or $(C)$, we have

$$
\left\|\mathcal{I} \mathcal{N}_{3}\left(v_{1}, v_{2} \cdot v_{3}\right)\right\|_{X^{0, b_{1}}} \lesssim R N_{(1)}^{-s} N_{(2)}^{-\delta_{0}} .
$$

(3) For any $N_{0} \gg N_{(1)}$,

$$
\left\|\Pi_{N_{0}}^{\perp} \mathcal{I} \mathcal{N}_{3}\left(v_{1}, v_{2} . v_{3}\right)\right\|_{X^{0, b_{1}}} \lesssim R N_{0}^{-s} N_{(2)}^{-\delta_{0}} .
$$

(4) If $N_{1} \gg N_{2}, N_{3}$ and $v_{1}$ is of type $(G)$ or $(C)$, then

$$
\left\|\Pi_{N_{1}}^{\perp} \mathcal{I} \mathcal{N}_{3}\left(v_{1}, v_{2}, v_{3}\right)\right\|_{X^{0, b_{1}}} \lesssim R N_{1}^{-s} N_{(2)}^{-\delta_{0}} .
$$

(5) The operator

$$
\mathcal{P}^{+}: v \mapsto \mathcal{I N}_{3}\left(v, v_{2}, v_{3}\right)
$$

satisfies

$$
\left\|\mathcal{P}^{+}\right\|_{X^{0, \frac{3}{8}} \rightarrow X^{0, b_{1}}} \lesssim R\left(N_{2} \vee N_{3}\right)^{-\delta_{0}}
$$

and similarly, the operator

$$
\mathcal{P}^{-}: v \mapsto \mathcal{I N}_{3}\left(v_{1}, v, v_{3}\right)
$$

satisfies

$$
\left\|\mathcal{P}^{-}\right\|_{X^{0, \frac{3}{8}} \rightarrow X^{0, b_{1}}} \lesssim R\left(N_{1} \vee N_{3}\right)^{-\delta_{0}}
$$

and the implicit constants are independent of $v_{2}, v_{3}$.

(6) When $L<N^{1-\delta}$, we have

$$
\left\|\mathcal{P}_{N, L}^{+} \circ\left(\widetilde{\mathbf{P}}_{N} \mathcal{H}\right)\right\|_{S^{b_{1}, q}} \lesssim R L^{-\delta_{0}}\|\mathcal{H}\|_{S^{b, q}},
$$

where $\widetilde{\mathbf{P}}_{N}=\mathcal{F}_{x}^{-1} \mathbf{1}_{\left|k_{1}\right| \sim N} \mathcal{F}_{x}$ is a Fourier projector similar as $\mathbf{P}_{N}$, and $\mathcal{P}_{N, L}^{+}$is given by (3.6).

(7) For all $L<N^{1-\delta}$,

$$
\left\|\mathcal{P}_{N, L}^{ \pm}\right\|_{X^{0, \frac{3}{8}} \rightarrow X^{0, b_{1}}} \lesssim R L^{-\delta_{0}} .
$$

(8) For the resonant terms, we have

$$
\begin{aligned}
& \left\|\mathcal{I N}_{0}\left(v_{1}, v_{2}, v_{3}\right)\right\|_{X^{0, b_{1}}} \lesssim R N_{(1)}^{-s} N_{(2)}^{-\delta_{0}}, \quad\left\|\Pi_{N_{0}}^{\perp} \mathcal{I} \mathcal{N}_{0}\left(v_{1}, v_{2}, v_{3}\right)\right\|_{X^{0, b_{1}}} \lesssim R N_{0}^{-s} N_{(2)}^{-\delta_{0}} \\
& \quad \text { for all } N_{0} \gg N_{(1)} .
\end{aligned}
$$


3.5. Proof of the main theorem. In this section, we assume Proposition 3.5, Proposition 3.6 and Proposition 3.7 and proceed to prove Proposition 3.3 .

Proof of Proposition 3.3. We assume $\operatorname{Loc}(M)$ is true for some large dyadic number $M$, and we will show that $\operatorname{Loc}(2 M)$ holds. To make the argument clean, first we delete a set of probability $<\mathrm{e}^{-2 M^{\theta} R^{\frac{2}{3}}}$ and we do not explicitly make any claim when there is necessary to delete some exceptional set of of the same size of the probability.

- Step 1: For $L=M, M<N^{1-\delta}$, we first show that (v),(vi), (vii) holds. From the decomposition

$$
v_{M}^{\#}=\psi^{M, \frac{1}{2}, \#}+\sum_{\frac{1}{2}<L<M^{1-\delta}} \zeta_{L}^{M, \#}+w_{M}^{\#},
$$

for any $w, \mathcal{P}_{N, M}^{+}(w)$ is a sum of $\chi_{T}(t) \mathcal{I} \Pi_{N} \mathcal{N}_{3}\left(w, v_{2}, v_{3}\right)$ for $v_{2}, v_{3}$ run over all terms of type $(\mathrm{G}),(\mathrm{C})$ or (D) with characterized parameters $\left(N_{2}, L_{2}\right),\left(N_{3}, L_{3}\right)$ satisfying

$$
\frac{M}{2} \leq N_{2} \vee N_{3} \leq M, \quad L_{j}<N_{j}^{1-\delta}<M, j=1,2 .
$$

Hence by (5) of Proposition 3.7, we have

$$
\left\|\mathcal{P}_{N, M}^{+}\right\|_{X^{0, b} \rightarrow X^{0, b}} \lesssim T^{b_{1}-b} R \sum_{\substack{N_{(2)}=\frac{M}{2}, M \\ N_{(3)} \leq M}} M^{-\delta_{0}} \lesssim T^{\frac{b_{1}-b}{2}} M^{-\delta_{0}^{2}},
$$

provided that $T \ll R^{-\frac{2}{b_{1}-b}}$ is chosen small enough. The kernel estimates (vi), (vii) are direct consequences of Proposition 3.6 and Proposition 3.5, with respectively.

-Step 2: Next we prove (i),(ii),(iii) and (iv) by using (3.5). Note that (ii),(iii) is a direct consequence of (i) and (iv), see Section 4 for details. From (3.5), we have

$$
\begin{aligned}
h^{N, M, \#} & =2 i \sum_{L \leq M} \mathcal{P}_{N, L}^{+} \circ h^{N, M, \#}+2 i \sum_{L<M} \mathcal{P}_{N, M}^{+} \circ h^{N, L, \#} \\
& +2 i \mathcal{P}_{N, M}^{+} \circ H^{N, \frac{1}{2}, \#} .
\end{aligned}
$$

Therefore,

$$
\begin{aligned}
\left\|h^{N, M, \#}\right\|_{Y^{b}} & \lesssim \sum_{L \leq M}\left\|\mathcal{P}_{N, L}^{+}\right\|_{X^{0, b} \rightarrow X^{0, b}}\left\|h^{N, M, \#}\right\|_{Y^{b}}+\sum_{L<M}\left\|\mathcal{P}_{N, M}^{+}\right\|_{X^{0, b} \rightarrow X^{0, b}}\left\|h^{N, L, \#}\right\|_{Y^{b}} \\
& +\left\|\mathcal{P}_{N, M}^{+}\right\|_{X^{0, b} \rightarrow X^{0, b}}\left\|H^{N, \frac{1}{2}, \#}\right\|_{Y^{b}} \\
& \lesssim\left\|h^{N, M, \#}\right\|_{Y^{b}} \sum_{L \leq M} T^{\theta} L^{-\delta_{0}^{2}}+\sum_{L<M} T^{\theta} M^{-\delta_{0}^{2}} L^{-\delta_{0}^{2}}+T^{\theta} M^{-\delta_{0}^{2}} .
\end{aligned}
$$

This implies that

$$
\left\|h^{N, M, \#}\right\|_{Y^{b}} \leq M^{-\delta_{0}^{2}}
$$

provided that $T>0$ is small enough. This proves the first inequality of (i). Next we prove (iv). From (3.5), we have

$\widetilde{h}_{k k^{*}}^{N, M, \#}(\lambda)=2 i \sum_{L \leq M} \int \sum_{k^{\prime}} \Theta_{k k^{\prime}}^{N, L}\left(\lambda, \lambda^{\prime}\right) \widetilde{h}_{k^{\prime} k^{*}}^{N, M, \#}\left(\lambda^{\prime}\right) d \lambda^{\prime}+2 i \sum_{L<M} \int \sum_{k^{\prime}} \Theta_{k k^{\prime}}^{N, M,}\left(\lambda, \lambda^{\prime}\right) \widetilde{h}_{k^{\prime} k^{*}}^{N, L, \#}\left(\lambda^{\prime}\right) d \lambda^{\prime}$

$$
+2 i \int \sum_{k^{\prime}} \Theta_{k k^{\prime}}^{N, M}\left(\lambda, \lambda^{\prime}\right) \widetilde{H}_{k^{\prime} k^{*}}^{N, \frac{1}{2}, \#}\left(\lambda^{\prime}\right) d \lambda^{\prime}
$$


Note that $\Theta_{k k^{\prime}}^{N, L}\left(t, t^{\prime}\right)$ is supported at $\left|k-k^{\prime}\right| \lesssim L$, from Lemma 2.14, we have

$$
\begin{aligned}
& \left\|\left\langle\frac{\left|k-k^{*}\right|}{M}\right\rangle^{\kappa}\langle\lambda\rangle^{b} \widetilde{h}_{k k^{*}}^{N, M, \#}(\lambda)\right\|_{L_{\lambda}^{2} l_{k, k^{*}}^{2}} \\
\lesssim & T^{b_{1}-b} \sum_{L \leq M}\left\|\langle\lambda\rangle^{b_{1}}\left\langle\lambda^{\prime}\right\rangle^{-b} \Theta_{k k^{\prime}}^{N, L}\left(\lambda, \lambda^{\prime}\right)\right\|_{L_{\lambda^{\prime}}^{2} l_{k^{\prime}}^{2} \rightarrow L_{\lambda}^{2} l_{k}^{2}}\left\|\left\langle\frac{\left|k^{\prime}-k^{*}\right|}{M}\right\rangle^{\kappa}\left\langle\lambda^{\prime}\right\rangle^{b} \widetilde{h}_{k^{\prime} k^{*}}^{N, M, \#}\left(\lambda^{\prime}\right)\right\|_{L_{\lambda^{\prime}}^{2} l_{k^{\prime}, k^{*}}^{2}} \\
+ & T^{b_{1}-b} \sum_{L<M}\left\|\langle\lambda\rangle^{b_{1}}\left\langle\lambda^{\prime}\right\rangle^{-b} \Theta_{k k^{\prime}}^{N, M}\left(\lambda, \lambda^{\prime}\right)\right\|_{L_{\lambda^{\prime}}^{2} l_{k^{\prime}}^{2} \rightarrow L_{\lambda}^{2} l_{k}^{2}}\left\|\left\langle\frac{\left|k^{\prime}-k^{*}\right|}{L}\right\rangle^{\kappa}\left\langle\lambda^{\prime}\right\rangle^{b} \widetilde{h}_{k^{\prime} k^{*}}^{N, L, \#}\left(\lambda^{\prime}\right)\right\|_{L_{\lambda^{\prime}}^{2} l_{k^{\prime}, k^{*}}^{2}} \\
+ & T^{b_{1}-b}\left\|\langle\lambda\rangle^{b_{1}}\left\langle\lambda^{\prime}\right\rangle^{-b} \Theta_{k k^{\prime}}^{N, M}\left(\lambda, \lambda^{\prime}\right)\right\|_{L_{\lambda^{\prime}}^{2} l_{k^{\prime}}^{2} \rightarrow L_{\lambda}^{2} l_{k}} \|\left\langle\left\langle k^{\prime}-k^{*}\right\rangle^{\kappa}\left\langle\lambda^{\prime}\right\rangle^{b} \widetilde{H}_{k^{\prime} k^{*}}^{N, \frac{1}{2}, \#}\left(\lambda^{\prime}\right) \|_{L_{\lambda^{\prime}}^{2}, l_{k^{\prime}, k^{*}}^{2}} .\right.
\end{aligned}
$$

By using the induction hypothesis and the boundeness of $\mathcal{P}_{N, L^{\prime}}^{+}$(the property (v) that we have just proved) for all $L^{\prime} \leq M$, we obtain (iv), provided that $T$ is chosen small enough.

Next we prove the third inequality of (i). From Lemma 2.15, we have

$$
\begin{aligned}
\left\|h^{N, M, \#}\right\|_{Z^{b}} & \lesssim T^{b_{1}-b} \sum_{L \leq M}\left\|\mathcal{P}_{N, L}^{+}\right\|_{X^{0, b} \rightarrow X^{0, b_{1}}}\left\|h^{N, M, \#}\right\|_{Z^{b}} \\
& +T^{b_{1}-b} \sum_{L<M} \min \left\{\left\|\Theta_{k k^{\prime}}^{N, M}\left(t, t^{\prime}\right)\right\|_{Z^{b_{1}, b}}\left\|h^{N, L, \#}\right\|_{Y^{b}},\left\|\mathcal{P}_{N, M}^{+}\right\|_{X^{0, b} \rightarrow X^{0, b_{1}}}\left\|h^{N, L, \#}\right\|_{Z^{b}}\right\} .
\end{aligned}
$$

Note that for the term

$$
\sum_{L<M} \int \sum_{k^{\prime}} \Theta_{k k^{\prime}}^{N, M}\left(\lambda, \lambda^{\prime}\right) \widetilde{h}_{k^{\prime} k^{*}}^{N, L}\left(\lambda^{\prime}\right) d \lambda^{\prime}
$$

in (3.18), we may assume that $\left|k-k^{*}\right| \ll N$ and $\left|k^{\prime}-k^{*}\right| \ll N$, since otherwise the bound follows trivially from (vi) that we have just proved. In particular, we have $|k|,\left|k^{\prime}\right| \geq \frac{N}{4}$ as $\left|k^{*}\right| \geq \frac{N}{2}$. Using (vi) that we have just proved, we obtain the third inequality of (i).

Finally in this step, we prove the second inequality of (i). Again in (3.18), we may assume that $|k|,\left|k^{\prime}\right| \geq \frac{N}{4}$ since otherwise we can use (vii) to obtain a better bound. After this reduction, we could apply (6) of Proposition 3.7 to treat the term

$$
\int \sum_{L \leq M} \sum_{k} \Theta_{k k^{\prime}}^{N, L}\left(\lambda, \lambda^{\prime}\right) \widetilde{h}_{k^{\prime} k^{*}}^{N, M, \#}\left(\lambda^{\prime}\right) d \lambda^{\prime}=2 i \sum_{L \leq M} \mathcal{P}_{N, L}^{+} \circ\left(\widetilde{\mathbf{P}}_{N} h^{N, M, \#}\right)_{k k^{*}}(\lambda) .
$$

Combining with Lemma 2.3, Lemma 2.14 and (6) or Proposition 3.7, we have

$$
\begin{aligned}
\left\|h^{N, M, \#}\right\|_{S^{b, q}} & \lesssim R T^{\frac{2\left(b_{1}-b\right)}{q^{\prime}}} \sum_{L \leq M} L^{-\delta_{0}}\left\|\widetilde{h}^{N, M, \#}\right\|_{S^{b, q}}+\sum_{L<M}\left\|\Theta^{N, M}\right\|_{S^{b, b, q}}\left\|h^{N, L, \#}\right\|_{Y^{b}} \\
& +\left\|\Theta^{N, M}\right\|_{S^{b, b, q}}\left\|H^{N, \frac{1}{2}, \#}\right\|_{Y^{b}},
\end{aligned}
$$

and this is conclusive when $T \ll 1$ is chosen small enough.

-Step 3: We prove (viii) by solving the equation (3.2). We will construct the fix-point of the equation (3.2) in the set $\mathcal{Z}_{2 M}$, where

$$
\mathcal{Z}_{N}:=\left\{w:\|w\|_{X^{0, b}} \leq N^{-s},\left\|\Pi_{N_{0}}^{\perp} w\right\|_{X^{0, b}} \leq N_{0}^{-s}, \forall N_{0} \geq N\right\}
$$

for dyadic numbers $N$. By hypothesis, we already know that $w_{N}^{\#} \in \mathcal{Z}_{N}$ for all $N \leq M$. For $N$, we define the norm

$$
\|w\|_{\mathcal{W}_{N}}:=\max \left\{N^{s}\|w\|_{X^{0, b}}, \sup _{N_{0} \geq N} N_{0}^{s}\left\|\Pi_{N_{0}}^{\perp} w\right\|_{X^{0, b}}\right\},
$$

where the sup is taken over all dyadic integers greater than $N$. Then finding a fix-point in the set $\mathcal{Z}_{2 M}$ is equivalent to find a fix-point in the unit ball of $\mathcal{W}_{2 M}$. Since it is not difficult to verify that (with $M$ fixed) $\|\cdot\|_{\mathcal{W}_{2 M}}$ is a norm on some Banach space embedded in $X^{0, b}$ (see Lemma 3.8), we can still apply the Banach fix-point theorem (contraction principle). 
First we verify that the mapping induced by the right side of (3.2) sends a unit ball of $\mathcal{W}_{2 M}$ to itself, provided that $T$ is sufficiently small (recall that $T$ is involved to define $\left.w^{\#}=w(t) \chi_{T}(t)\right)$. Thanks to Lemma 2.1, it suffices to estimate the $X^{0, b_{1}}$ norm for the multi-linear terms without the time cutoff $\chi_{T}(t)$ factor in front of the Duhamel operator $\mathcal{I}$. From (3.2) (changing $w_{2 M}^{\#}$ there to $\left.w^{\#}\right)$, the right side of the integration equation of $w^{\#}(t)$ is a linear combination of multi-linear terms of types 1)-7) below (3.2). Since for $\delta \ll 1$, $M<(2 M)^{1-\delta}$, all the conditions (3.10), (3.11), (3.12) and (3.16) are satisfies for type (C) terms with characterized parameters $\left(N_{j}, L_{j}\right)$ satisfying $N_{j} \leq 2 M$ and $L_{j}<N_{j}^{1-\delta}$. Moreover,

$$
\left\|w_{N_{j}}^{\#}\right\|_{X^{0, b}} \leq N_{j}^{-s},\left\|\Pi_{N_{0}}^{\perp} w_{N_{j}}\right\|_{X^{0, b}} \leq N_{0}^{-s}
$$

for all $N_{j} \leq M$ and $N_{0} \geq N_{j}$. Then the rest argument is a direct application of the statements in Proposition 3.7. Next, to verify that the mapping defined by the right side of (3.2) is a contraction, the argument is similar. Indeed, we pick two different $w, w^{\prime} \in \mathcal{Z}_{2 M}$, due to the tri-linearity of the right side of $(3.2)$, there must be $w-w^{\prime}$ appearing in at least one place in each multi-linear expression $\mathcal{N}(\cdot, \cdot, \cdot)$. Then applying (5) of Proposition 3.7 and Lemma 2.1, we are able to leave out a factor $T^{b_{1}-b}\left\|w-w^{\prime}\right\|_{\mathcal{W}_{2 M}}$ when estimating the $\mathcal{W}_{2 M}$ norm of the difference. From the Banach fix-point theorem, we are able to find the unique fix-point $w^{\#}(t)=w(t) \chi_{2 T}(t)$ in $\mathcal{Z}_{2 M}$, supported in $|t| \leq 2 T$.

The proof of Proposition 3.3 is now complete.

Lemma 3.8. Assume that $\mathcal{W}$ is a Banach space with the norm $\|\cdot\|$ and $\left(T_{j}\right)_{j \in \mathbb{N}}$ is a sequence of bounded linear operators on $\mathcal{X}$ and $T_{1}=\mathrm{Id}$. Consider another space

$$
\mathcal{W}_{*}:=\left\{w \in \mathcal{W}:\|w\|_{*}<+\infty\right\}
$$

where

$$
\|w\|_{*}:=\sup _{j \in \mathbb{N}}\left\|T_{j} w\right\| .
$$

Then with $\left(\mathcal{W}_{*},\|\cdot\|_{*}\right)$ is a Banach space.

Proof. The triangle inequality is trivial. We only need to show that $\mathcal{W}_{*}$ is complete. Take a Cauchy-sequence $\left(w^{(k)}\right) \subset \mathcal{W}_{*}$ such that

$$
\lim _{k, k^{\prime} \rightarrow \infty}\left\|w^{(k)}-w^{\left(k^{\prime}\right)}\right\|_{*}=0 .
$$

In particular, since $T_{1}=\operatorname{Id}$ and $\mathcal{W}$ is complete, there exists $w \in \mathcal{W}$ such that $\left\|w^{(k)}-w\right\| \rightarrow$ 0 . Since for any $\epsilon>0$, there exists $k_{0}=k_{0}(\epsilon)$, such that for all $k, k^{\prime} \geq k_{0}$,

$$
\sup _{j \in \mathbb{N}}\left\|T_{j} w^{(k)}-T_{j} w^{\left(k^{\prime}\right)}\right\|<\epsilon .
$$

Thus for each fixed $j$, passing $k^{\prime} \rightarrow+\infty$, we have $\left\|T_{j} w^{(k)}-T_{j} w\right\| \leq \epsilon$. This implies that

$$
\lim _{k \rightarrow \infty} \sup _{j \in \mathbb{N}}\left\|T_{j} w^{(k)}-T_{j} w\right\|=0 .
$$

The proof of Lemma 3.8 is complete.

3.6. Sketch of the convergence of the whole sequence. We now explain briefly how to modify the arguments in this section to prove the convergence for the whole sequence $\left(v_{n}\right)_{n \in \mathbb{N}}$, satisfying

$$
i \partial_{t} v_{n}+\left|D_{x}\right|^{\alpha} v_{n}=\mathcal{N}\left(v_{n}\right),\left.\quad v_{n}\right|_{t=0}=\left(\Pi_{n}-\Pi_{\frac{N}{2}}\right) \phi^{\omega},
$$

where $\frac{N}{2}<n<N$. For this, we first define similar random averaging operator (as well as their kernels): $H^{n, L}, h^{n, L}, \mathcal{P}_{n, L}^{ \pm}, \Theta_{k k^{\prime}}^{n, L}$ and the corresponding "para-controlled" objects $\psi_{L}^{n}, \zeta_{L}^{n}=\psi_{L}^{n}-\psi_{\frac{L}{2}}^{n}$ by changing $N$ to $n$ while keeping the constraint $L<N^{1-\delta}$. Then we add the same bounds for these objects as $H^{N, L}, h^{N, L}, \mathcal{P}_{N, L}^{ \pm}, \Theta_{k k^{\prime}}^{N, L}, \psi_{L}^{N}, \zeta_{L}^{N}$ in the definition of $\operatorname{Loc}(M)$ for all $(L, N)$ such that $L<M, L<N^{1-\delta}$ and all $\frac{N}{2}<n<N$. We need 
also to add $X^{0, b}$ bounds of $w_{n^{\prime}}$ and $\Pi_{N_{0}}^{\perp} w_{n^{\prime}}$ for all $\frac{N^{\prime}}{2}<n^{\prime}<N^{\prime}, N_{0} \geq N^{\prime}$ and $N^{\prime} \leq M$ in the definition of $\operatorname{Loc}(M)$. Then to pass from $\operatorname{Loc}(M)$ to $\operatorname{Loc}(2 M)$, we make use of Proposition 3.5. Proposition 3.6 and Proposition 3.7. Note that here we should prove stronger statements in these propositions accordingly, providing estimates of $S^{b_{1}, b, q}$ and $Z^{b_{1}, b}$ norms of the kernel $\Theta_{k, k_{1}}^{n, L}$. Here the observation is that, the proof of Proposition 3.5 and Proposition 3.6 (in Section 7) is not specific to dyadic numbers $N$ (in the definition of $\Theta^{n, L}$, the letter $n$ appears only in the frequency truncation $\Pi_{n}$ in front of the multi-linear expression $\mathcal{N}_{3}$ ) and the probability of the exceptional set that we delete each time can depend only on the dyadic parameters $L, N$ such that $L<N^{1-\delta}$. Therefore, the results of Proposition 3.5 and Proposition 3.6 are also true for all $\Theta_{k, k_{1}}^{n, L}$ such that $\frac{N}{2}<n<N$ and $L<N^{1-\delta}$. Finally, to get desired bounds for $w_{m}$ if $M<m<2 M$, the analysis is similar as solving the Fix-point 2 for $w_{2 M}$. Therefore, if $\frac{N}{2}<n<N$, through the decomposition

$$
v_{n}(t)=v_{\frac{N}{2}}(t)+S(t)\left(\Pi_{n}-\Pi_{\frac{N}{2}}\right) \phi^{\omega}+\sum_{\frac{1}{2}<L \leq L_{N / 2}} \zeta_{L}^{n}+w_{n}(t),
$$

we deduce that $\left(v_{n}(t)\right)_{n \in \mathbb{N}}$ is also a Cauchy sequence in $C\left([-T, T] ; H^{\sigma_{0}}(\mathbb{T})\right)$.

Once Theorem 3 is proved, we are able to deduce Theorem 2 as in [28], and we omit the detail.

\section{4. $L^{\infty}$ AND Fourier-LEBESGUE PROPERTY FOR PARACONTROLLED OBJECTS}

In this section, we prove (ii) (iii) of the statement $\operatorname{Loc}(2 M)$. Note that $\epsilon_{2}>\theta+2 \epsilon_{1}$, the key for the proof of (iii) is the following probabilistic pointwise bound:

Lemma 4.1. Assume that $0<T<1, \frac{1}{2}<L_{1}<N_{1}^{1-\delta}$ and

$$
\left\|h^{N_{1} L_{1}}\right\|_{Z^{b}} \leq N_{1}^{-(\alpha-1)+\epsilon_{1}} L_{1}^{-\nu}, \quad\left\|\left\langle\frac{\left|k-k^{*}\right|}{L_{1}}\right\rangle^{\kappa}\langle\lambda\rangle^{b} \widetilde{h}_{k k^{*}}^{N_{1} L_{1}}(\lambda)\right\|_{L_{\lambda}^{2} l_{k, k^{*}}^{2}} \leq N_{1},
$$

then for any $R \gg \epsilon_{1}^{-\frac{1}{2}}$, outside a set of probability $<\mathrm{e}^{-c N_{1}^{\theta} R^{2}}$, we have

$$
\left\|\sum_{\left|k_{1}\right| \leq N_{1}} \sum_{\left|k_{1}^{*}\right| \sim N_{1}} \chi_{T}(t) h_{k_{1} k_{1}^{*}}^{N_{1} L_{1}}(t) \frac{g_{k_{1}^{*}}}{\left[k_{1}^{*}\right]^{\frac{\alpha}{2}}} \mathrm{e}^{i k_{1} x}\right\|_{L_{t}^{4} L_{x}^{\infty}} \leq C_{\epsilon} T^{\frac{1}{8}} R N_{1}^{-(\alpha-1)+\theta+2 \epsilon_{1}} L_{1}^{\frac{1}{2}-\nu} .
$$

Proof. By abusing notation, we still denote by $h^{N L}(t)=\chi_{T}(t) h^{N L}(t)$. Assume that $\epsilon_{1}^{-1}<q_{1}<\infty$, then from Chebyshev's inequality, Sobolev embedding $W_{x}^{\frac{2}{q_{1}}, q_{1}} \hookrightarrow L_{x}^{\infty}$ and Minkowski's inequality, we have

$$
\begin{aligned}
& \mathbb{P}\left[\left\|\sum_{\left|k_{1}\right| \leq N_{1},\left|k_{1}^{*}\right| \sim N_{1}} h_{k_{1} k_{1}^{*}}^{N_{1} L_{1}}(t) \frac{g_{k_{1}^{*}}}{\left[k_{1}^{*}\right]^{\frac{\alpha}{2}}} \mathrm{e}^{i k_{1} x}\right\|_{L_{t}^{4} L_{x}^{\infty}}>A\right] \\
\leq & \frac{1}{A^{q_{1}}}\left\|\sum_{\left|k_{1}\right| \leq N_{1},\left|k_{1}^{*}\right| \sim N_{1}} h_{k_{1} k_{1}^{*}}^{N_{1} L_{1}}(t) \frac{g_{k_{1}^{*}}}{\left[k_{1}^{*}\right]^{\frac{\alpha}{2}}} \mathrm{e}^{i k_{1} x}\right\|_{L_{\omega}^{q_{1}} L_{t}^{4} L_{x}^{\infty}}^{q_{1}} \\
\leq & \frac{1}{A^{q_{1}}}\left\|\sum_{\left|k_{1}\right| \leq N_{1},\left|k_{1}^{*}\right| \sim N_{1}} h_{k_{1} k_{1}^{*} N_{1} L_{1}}(t) \frac{\left\langle k_{1}\right\rangle^{\frac{2}{q_{1}}}}{\left[k_{1}^{*}\right]^{\frac{\alpha}{2}}} \mathrm{e}^{i k_{1} x}\right\|_{L_{t}^{4} L_{x}^{q_{1}} L_{\omega}^{q_{1}}}^{q_{1}} .
\end{aligned}
$$

Since $g_{k_{1}^{*}}(\omega)$ and $h_{k_{1} k_{1}^{*}}^{N_{1} L_{1}}(\omega)$ are independent, we may write them as $g_{k_{1}^{*}}\left(\omega_{1}\right)$ and $h_{k_{1} k_{1}^{*}}^{N_{1} L_{1}}\left(\omega_{2}\right)$. Then for fixed $t$ and $x$, we have from Lemma 2.16 that

$$
\begin{aligned}
\left\|\sum_{\left|k_{1}^{*}\right| \sim N_{1}} \frac{g_{k_{1}^{*}}(\omega)}{\left[k_{1}^{*}\right]^{\frac{\alpha}{2}}} \sum_{\left|k_{1}\right| \leq N_{1}}\left\langle k_{1}\right\rangle^{\frac{2}{q_{1}}} h_{k_{1} k_{1}^{*}}^{N_{1} L_{1}}(t) \mathrm{e}^{i k_{1} x}\right\|_{L_{\omega_{1}}^{q_{1}}} & \lesssim q_{1}^{\frac{1}{2}}\left\|\sum_{\left|k_{1}^{*}\right| \sim N_{1}} \frac{g_{k_{1}^{*}}(\omega)}{\left[k_{1}^{*}\right]^{\frac{\alpha}{2}}} \sum_{\left|k_{1}\right| \leq N_{1}}\left\langle k_{1}\right\rangle^{\frac{2}{q_{1}}} h_{k_{1} k_{1}^{*}}^{N_{1} L_{1}}(t) \mathrm{e}^{i k_{1} x}\right\|_{L_{\omega_{1}}^{2}} \\
& \lesssim q_{1}^{\frac{1}{2}} N_{1}^{-\frac{\alpha}{2}+\frac{2}{q_{1}}}\left(\sum_{\left|k_{1}^{*}\right| \sim N_{1}}\left|\sum_{\left|k_{1}\right| \leq N_{1}} h_{k_{1} k_{1}^{*}}^{N_{1} L_{1}}(t)\right|^{2}\right)^{\frac{1}{2}}
\end{aligned}
$$


Note that

$$
\begin{aligned}
& \left(\sum_{\left|k_{1}^{*}\right| \sim N_{1}}\left|\sum_{\left|k_{1}\right| \leq N_{1}} h_{k_{1} k_{1}^{*}}^{N_{1} L_{1}}(t)\right|^{2}\right)^{\frac{1}{2}} \leq\left(L_{1} N_{1}^{\epsilon_{1}}\right)^{\frac{1}{2}}\left(\sum_{\left|k_{1}^{*}\right| \sim N_{1},\left|k_{1}-k_{1}^{*}\right| \leq L_{1} N_{1}^{\epsilon_{1}}}\left|h_{k_{1} k_{1}^{*}}^{N_{1} L_{1}}(t)\right|^{2}\right)^{\frac{1}{2}} \\
& +N_{1}^{1-\epsilon_{1} \kappa}\left(\sum_{\substack{\left|k_{1}^{*}\right| \sim N_{1},\left|k_{1}\right| \leq N_{1},\left|k_{1}^{*}-k_{1}\right|>L_{1} N_{1}^{\epsilon}}}\left\langle\frac{\left|k_{1}-k_{1}^{*}\right|}{L}\right\rangle^{2 \kappa}\left|h_{k_{1} k_{1}^{*}}^{N_{1} L_{1}}(t)\right|^{2}\right)^{\frac{1}{2}} .
\end{aligned}
$$

Therefore,

$$
\begin{aligned}
& \mathbb{P}\left[\left\|\sum_{\left|k_{1}\right| \leq N_{1},\left|k_{1}^{*}\right| \sim N_{1}} h_{k_{1} k_{1}^{*}}^{N_{1} L_{1}}(t) \frac{g_{k_{1}^{*}}}{\left|k_{1}^{*}\right|^{\frac{\alpha}{2}}} \mathrm{e}^{i k_{1} x}\right\|_{L_{t}^{4} L_{x}^{\infty}}>A\right] \\
\lesssim & \frac{q_{1}^{\frac{q_{1}}{2}} N_{1}^{-\frac{q_{1} \alpha}{2}+2}}{A^{q_{1}}}\left[\left(L_{1} N_{1}^{\epsilon_{1}}\right)^{\frac{q_{1}}{2}}\left\|h_{k_{1} k_{1}^{*}}^{N_{1} L_{1}}(t)\right\|_{L_{t}^{4} l_{k_{1}, k_{1}^{*}}^{2}}^{q_{1}}+N_{1}^{\frac{q_{1}}{2}-q_{1} \epsilon_{1} \kappa}\left\|\left\langle\frac{\left|k_{1}-k_{1}^{*}\right|}{L}\right\rangle^{\kappa} h_{k_{1} k_{1}^{*}}^{N_{1} L_{1}}(t)\right\|_{L_{t}^{4} l_{k_{1}, k_{1}^{*}}^{2}}^{q_{1}}\right] .
\end{aligned}
$$

using again the Minkowski inequality and the Sobolev embedding $H_{t}^{\frac{1}{4}} \hookrightarrow L_{t}^{4}$, for any $h_{k_{1} k_{1}^{*}}(t)$, we have

$$
\begin{aligned}
\left\|h_{k_{1} k_{1}^{*}}^{N_{1} L_{1}}(t)\right\|_{L_{t}^{4} l_{k_{1}, k_{1}^{*}}^{2}} & =\left\|\mathrm{e}^{i t\left|k_{1}\right|^{\alpha}} h_{k_{1} k_{1}^{*}}^{N_{1} L_{1}}(t)\right\|_{L_{t}^{4} l_{k_{1}, k_{1}^{*}}^{2}} \\
& \leq\left\|\mathrm{e}^{i t\left|k_{1}\right|^{\alpha}} h_{k_{1} k_{1}^{*}}^{N_{1} L_{1}}(t)\right\|_{l_{k_{1}, k_{1}^{*}}^{2} H_{t}^{\frac{1}{4}}}=\left\|\left\langle\lambda_{1}\right\rangle^{\frac{1}{4}} \widetilde{h}_{k_{1} k_{1}^{*}}^{N_{1} L_{1}}\left(\lambda_{1}\right)\right\|_{l_{k_{1}, k_{1}^{*}}^{2} L_{\lambda}^{2}} \lesssim T^{\frac{1}{8}}\left\|h_{k_{1}, k_{1}^{*}}^{N_{1} L_{1}}\right\|_{Z^{b}},
\end{aligned}
$$

where to the last step, we use the fact that $h_{k_{1} k_{1}^{*}}^{N_{1} L_{1}}(t)=\chi_{T}(t) h_{k_{1} k_{1}^{*}}^{N_{1} L_{1}}(t)$ and the timelocalization property (Lemma 2.3). Similarly,

$$
\left\|\left\langle\frac{\left|k_{1}-k_{1}^{*}\right|}{L}\right\rangle^{\kappa} h_{k_{1} k_{1}^{*}}^{N_{1} L_{1}}(t)\right\|_{L_{t}^{4} l_{k_{1}, k_{1}^{*}}^{2}} \lesssim T^{\frac{1}{8}}\left\|\left\langle\frac{\left|k_{1}-k_{1}^{*}\right|}{L}\right\rangle^{\kappa} h_{k_{1} k_{1}^{*}}^{N_{1} L_{1}}(t)\right\|_{Z^{b}}
$$

Therefore,

$$
\begin{aligned}
& \mathbb{P}\left[\left\|\sum_{\left|k_{1}\right| \leq N_{1},\left|k_{1}^{*}\right| \sim N_{1}} h_{k_{1} k_{1}^{*}}^{N_{1} L_{1}}(t) \frac{g_{k_{1}^{*}}}{\left|k_{1}^{*}\right|^{\frac{\alpha}{2}}} \mathrm{e}^{i k_{1} x}\right\|_{L_{t}^{4} L_{x}^{\infty}}>A\right] \\
\lesssim & \frac{q_{1}^{\frac{q_{1}}{2}}}{A^{q_{1}}} N_{1}^{-\frac{q_{1} \alpha}{2}+2} T^{\frac{q_{1}}{8}}\left[L_{1}^{\frac{q_{1}}{2}-q_{1} \nu} N_{1}^{q_{1}\left(1-\frac{\alpha}{2}+2 \epsilon_{1}\right)}+N_{1}^{q_{1}\left(1-\epsilon_{1} \kappa\right)}\right] \lesssim \frac{q_{1}^{\frac{q_{1}}{2}}}{A^{q_{1}}} N_{1}^{q_{1}(1-\alpha)+2 q_{1} \epsilon} T^{\frac{q_{1}}{8}} L_{1}^{q_{1}\left(\frac{1}{2}-\nu\right)} .
\end{aligned}
$$

Since $\kappa \epsilon_{1} \gg 1$, by choosing $R=R N_{1}^{\theta} T^{\frac{1}{8}} N_{1}^{-(\alpha-1)+2 \epsilon_{1}} L_{1}^{\frac{1}{2}-\nu}$ and optimizing the choice of $q_{1} \sim R^{2}$ (thanks to the fact that $R \gg \epsilon_{1}^{-1 / 2}$ ), we obtain the desired estimate. This completes the proof of Lemma 4.1 .

Similarly, to prove (ii) for $\operatorname{Loc}(2 M)$, it suffices to prove:

Lemma 4.2. Assume that $0<T<1, \frac{1}{2}<L_{1}<N_{1}^{1-\delta}$ and

$$
\left\|h^{N_{1} L_{1}}\right\|_{Z^{b}} \leq C N_{1}^{-(\alpha-1)+\epsilon_{1}} L_{1}^{-\nu}, \quad\left\|\left\langle\frac{\left|k-k^{*}\right|}{L_{1}}\right\rangle^{\kappa}\langle\lambda\rangle^{b} \widetilde{h}_{k k^{*}}^{N_{1} L_{1}}(\lambda)\right\|_{L_{\lambda}^{2} l_{k, k^{*}}^{2}} \leq N_{1}^{\frac{1}{2}}
$$

and

$$
\left\|h^{N_{1} L_{1}}\right\|_{S^{b, q}} \leq N_{1}^{-\frac{\alpha}{2}+\epsilon_{1}} L_{1}^{-\nu} .
$$

Let

$$
v_{1}(t, x)=\sum_{\left|k_{1}\right| \leq N_{1}} \sum_{\left|k_{1}^{*}\right| \sim N_{1}} \chi_{T}(t) h_{k_{1} k_{1}^{*}}^{N_{1} L_{1}}(t) \frac{g_{k_{1}^{*}}}{\left[k_{1}^{*}\right]^{\frac{\alpha}{2}}} e^{i k_{1} x}
$$

Then for any $R \gg \epsilon_{1}^{-\frac{1}{2}}$, outside a set of probability $<\mathrm{e}^{-c N_{1}^{\theta} R^{2}}$, we have

$$
\left\|v_{1}\right\|_{\substack{0, \frac{2 b_{0}}{q^{\prime}} \\ X_{\infty} q^{\prime}}}=\left\|\langle\lambda\rangle^{\frac{2 b_{0}}{q^{\prime}}} \widetilde{v}(\lambda, k)\right\|_{l_{k}^{\infty} L_{\lambda}^{q}} \lesssim T^{b-b} R N_{1}^{-\frac{\alpha}{2}+\epsilon_{1}+\theta+\frac{1}{q}} L_{1}^{-\nu}
$$


and

$$
\left\|v_{1}\right\|_{X^{0, b_{0}}} \lesssim T^{b-b_{0}} R N_{1}^{-(\alpha-1)+\epsilon_{1}+\theta} L_{1}^{-\nu}
$$

Proof. We only prove the bound $X_{\infty, q}^{0, \frac{2 b_{0}}{q^{\prime}}}$ and the bound for the norm $X^{0, b_{0}}$ can be obtained in the similar way. Since

$$
\widetilde{v}_{1}\left(\lambda_{1}, k_{1}\right)=\sum_{k_{1}^{*}:\left|k_{1}^{*}\right| \sim N_{1}} \widetilde{h}_{k_{1} k_{1}^{*}}^{N_{1} L_{1}}\left(\lambda_{1}\right) \frac{g_{k^{*}}}{\left[k_{1}^{*}\right]^{\frac{\alpha}{2}}} .
$$

Note that for fixed $\left|k_{1}\right| \leq N_{1}$, applying Corollary 2.17, outside a set $\Omega_{k_{1}}$ (depending on $k_{1}$ ) of probability $<\mathrm{e}^{-c N_{1}^{\theta} R_{1}^{2}}$,

$$
\left|\sum_{k_{1}^{*}:\left|k_{1}^{*}\right| \sim N_{1}} \widetilde{h}_{k_{1} k_{1}^{*}}^{N_{1} L_{1}}\left(\lambda_{1}\right) \frac{g_{k_{1}^{*}}}{\left[k_{1}^{*}\right]^{\frac{\alpha}{2}}}\right| \leq C N^{\theta} R \cdot\left(\mathbb{E}^{\mathcal{B}_{\leq L}}\left[\left|\sum_{k_{1}^{*}:\left|k_{1}^{*}\right| \sim N} \widetilde{h}_{k_{1} k_{1}^{*}}^{N_{1} L_{1}}(\lambda) \frac{g_{k_{1}^{*}}}{\left[k_{1}^{*}\right]^{\frac{\alpha}{2}}}\right|^{2}\right]\right)^{\frac{1}{2}} .
$$

By deleting the union $\cup_{\left|k_{1}\right| \leq N_{1}} \Omega_{k_{1}}$ for which the probability is smaller than

$$
\sum_{\left|k_{1}\right| \leq N_{1}} \mathrm{e}^{-c N_{1}^{\theta} R^{2}}<\mathrm{e}^{-c^{\prime} N_{1}^{\theta} R^{2}}
$$

above bound (4.1) is uniform for $\left|k_{1}\right| \leq N_{1}$. Using the independence of $h_{k_{1} k_{1}^{*}}^{N_{1} L_{1}}$ and $g_{k_{1}^{*}}$, the conditional expectation can be bounded by

$$
N_{1}^{-\alpha+\theta} R\left\|\widetilde{h}_{k_{1} k_{1}^{*}}^{N_{1} L_{1}}(\lambda)\right\|_{l_{k_{1}}^{\infty} l_{k_{1}^{*}}^{2}} \leq N_{1}^{-\alpha+\theta} R\left\|\widetilde{h}_{k_{1} k_{1}^{*}}^{N_{1} L_{1}}(\lambda)\right\|_{l_{k}^{q} l_{k^{*}}^{2}} \cdot
$$

Noticing that $k_{1}$ is constraint in the set $\left|k_{1}\right| \leq N_{1}$, multiplying by $\langle\lambda\rangle^{\frac{2 b_{0}}{q^{\prime}}}$ to both sides of (4.1) and taking the $L_{\lambda_{1}}^{q}$ and then $l_{k_{1}}^{\infty}$, we have

$$
\left\|v_{1}\right\|_{\substack{0, \frac{2 b_{0}}{q^{\prime}} \\ X_{\infty, q}}} \leq N_{1}^{-\alpha+\theta+\frac{1}{q}} R\left\|\langle\lambda\rangle^{\frac{2 b_{0}}{q^{\prime}}} \widetilde{h}_{k_{1} k_{1}^{*}}^{N_{1} L_{1}}\left(\lambda_{1}\right)\right\|_{l_{k_{1}}^{\infty} L_{\lambda_{1}}^{q} l_{k_{1}^{*}}^{2}}=N_{1}^{-\alpha+\theta+\frac{1}{q}} R\left\|h^{N_{1} L_{1}}\right\|_{S^{b_{0}, q}} .
$$

Using Lemma 2.3, we have $\left\|h^{N_{1} L_{1}}\right\|_{S^{b_{0}, q}} \lesssim T^{b-b_{0}}\left\|h^{N_{1} L_{1}}\right\|_{S^{b, q}}$ since $\left.h^{N_{1} L_{1}}\right|_{t=0}=0$. The proof of Lemma 4.2 is now complete.

\section{Mapping properties of the operator $\mathcal{P}_{N, L}^{+}$}

In this section, we will prove $(3),(5),(6),(7)$ of Proposition 3.7

For given space-time functions $v_{2}, v_{3}$, consider the operator

$$
\mathcal{Q}_{3, N}(w):=\mathcal{I} \Pi_{N} \mathcal{N}_{3}\left(w, v_{2}, v_{3}\right)
$$

and we denote by $\Theta_{k k^{\prime}}\left(t, t^{\prime}\right)$ its kernel. Note that the operator $\mathcal{Q}_{3, N}$ depends on the functions $v_{2}, v_{3}$ and $N$. By implicitly inserting $w=\chi(t) w$, we have from Lemma 2.4 that

$$
\left.\widetilde{\mathcal{Q}_{3, N}}(w)(\lambda, k)=\int_{\mathbb{R}} K(\lambda, \mu) \widetilde{\left(\mathcal{N}_{3}\right.}\left(w, v_{2}, v_{3}\right)\right)(\mu, k) d \mu
$$

Note that

$$
\begin{aligned}
& \widetilde{\mathcal{N}_{3}}\left(w, v_{2}, v_{3}\right)(\lambda, k) \\
= & \frac{1}{(2 \pi)^{3}} \sum_{\substack{|k| \leq N, k_{1}, k_{2}, k_{3} \\
\left(k_{1}, k_{2}, k_{3}\right) \in \Gamma(k)}} \int_{\chi} \widehat{\chi}\left(\lambda-\lambda_{1}+\lambda_{2}-\lambda_{3}-\Phi_{k_{1}, k_{2}, k_{3}}\right) \widetilde{w}\left(\lambda_{1}, k_{1}\right) \overline{v_{2}}\left(\lambda_{2}, k_{2}\right) \widetilde{v_{3}}\left(\lambda_{3}, k_{3}\right) d \lambda_{1} d \lambda_{2} d \lambda_{3} .
\end{aligned}
$$

Denote by

$$
\Xi_{k k^{\prime}}\left(\lambda, \lambda^{\prime}\right):=\frac{\mathbf{1}_{k \neq k^{\prime},\left|k^{\prime}\right| \leq N}}{(2 \pi)^{3}} \sum_{\substack{k_{2}, k_{3} \\ k_{2} \neq k_{3}, k_{2}-k_{3}=k-k^{\prime}}} \int \widehat{\chi}\left(\lambda-\lambda^{\prime}+\lambda_{2}-\lambda_{3}-\Phi_{k^{\prime}, k_{2}, k_{3}}\right) \overline{v_{2}}\left(\lambda_{2}, k_{2}\right) \widetilde{v_{3}}\left(\lambda_{3}, k_{3}\right) d \lambda_{2} d \lambda_{3},
$$


then from Lemma 2.4 , the kernel of $\mathcal{Q}_{3, N}$ is given by

$$
\widetilde{\Theta}_{k k^{\prime}}\left(\lambda, \lambda^{\prime}\right)=\int_{\mathbb{R}} K(\lambda, \mu) \Xi_{k k^{\prime}}\left(\mu, \lambda^{\prime}\right) d \mu .
$$

5.1. $S^{b, q}$-mapping properties of the operator $\mathcal{P}_{N, L}^{+}$. In this subsection, we prove (6) of Proposition 3.7. By decomposing $v_{L}^{\#}$ as sums of type $(\mathrm{G}),(\mathrm{C})$ and $(\mathrm{D})$ terms in $\mathcal{P}_{N, L}^{+}$, it suffices to prove the estimate by changing $\mathcal{P}_{N, L}^{+}$to some operator

$$
w \mapsto \Theta_{N}(w):=\Pi_{N} \mathcal{I} \mathcal{N}_{3}\left(w, v_{2}, v_{3}\right)
$$

for functions $v_{2}=\Pi_{L} v_{2}, v_{3}=\Pi_{L} v_{3}$ of type (G),(C) or (D) with characterized parameters $\left(N_{2}, L_{2}\right),\left(N_{3}, L_{3}\right)$ satisfying $N_{2} \vee N_{3}=L$ or $\frac{L}{2}$ and $N_{2}, N_{3} \ll N$. We denote by $\left(\Theta_{k k_{1}}\left(t, t^{\prime}\right)\right)$ the kernel of $\Theta_{N}$. Let $\mathcal{H}(t)$ be a linear operator with kernel $\left(H_{k k^{*}}(t)\right)$. By abusing the notation, we still denote by $q_{k k^{*}}(t)$ the matrix-element of the operator $\Theta_{N} \circ\left(\widetilde{\mathbf{P}}_{N} \mathcal{H}(\lambda)\right)$. Therefore,

$$
\widetilde{q}_{k k^{*}}(\lambda)=\int_{\mathbb{R}} \sum_{\left|k_{1}\right| \sim N} \widetilde{\Theta}_{k k_{1}}\left(\lambda, \lambda_{1}\right) \widetilde{H}_{k_{1} k^{*}}\left(\lambda_{1}\right) d \lambda_{1} .
$$

Note that on the support of $\widetilde{H}_{k_{1} k^{*}}\left(\lambda_{1}\right)$, we have $\left|k_{1}\right| \sim N$. Inserting (5.1) and (5.2) into the expression above, we have

$$
\begin{aligned}
\widetilde{q}_{k k^{*}}(\lambda)= & \sum_{\substack{\left(k_{1}, k_{2}, k_{3}\right) \in \Gamma \times \\
\left|k_{1}\right| \sim N \\
\left|k_{j}\right| \leq N_{j}, j=2,3}} \int_{k} K(\lambda, \mu) \widetilde{H}_{k_{1} k^{*}}\left(\lambda_{1}\right) d \mu d \lambda_{1} \\
& \times \int \widehat{\chi}\left(\mu-\lambda_{1}+\lambda_{2}-\lambda_{3}+\Phi_{k_{1}, k_{2}, k_{3}}\right) \overline{\widetilde{v}_{2}}\left(\lambda_{2}, k_{2}\right) \widetilde{v}_{3}\left(\lambda_{3}, k_{3}\right) d \lambda_{2} d \lambda_{3},
\end{aligned}
$$

where the kernel $|K(\lambda, \mu)| \lesssim\langle\mu\rangle^{-1}\left(\langle\lambda\rangle^{-10}+\langle\lambda-\mu\rangle^{-10}\right)$. Our goal is to estimate

$$
\left\|\langle\lambda\rangle^{\frac{2 b_{1}}{q^{\prime}}} \widetilde{q}_{k k^{*}}(\lambda)\right\|_{l_{k}^{\infty} L_{\lambda}^{q} l_{k^{*}}^{2}}
$$

We will control it in two ways, according to the size of $L$.

-Case 1: $L^{\frac{2 b_{1}}{q^{\prime}}-\frac{\alpha-1}{2}+\epsilon_{2}+\delta_{0}} \lesssim N^{\frac{(\alpha-1)\left(q^{\prime}-2 b_{1}\right)}{q^{\prime}}}$

In this case, we will integrate high modulations first.

By taking $l_{k^{*}}^{2}$ and using Minkowski, Hölder, we have (here we hide the constraints on $k_{j}$ by implicitly inserting some indicators to $\widetilde{H}_{k_{1} k^{*}}, \widetilde{v}_{2}$, and $\widetilde{v}_{3}$ )

$$
\left\|\widetilde{q}_{k k^{*}}(\lambda)\right\|_{l_{k^{*}}} \lesssim \sum_{\left(k_{1}, k_{2}, k_{3}\right) \in \Gamma(k)} \int \frac{1}{\langle\mu\rangle}\left(\frac{1}{\langle\lambda\rangle^{10}}+\frac{1}{\langle\lambda-\mu\rangle^{10}}\right) \frac{a_{1}\left(k_{1}\right) a_{2}\left(k_{2}\right) a_{3}\left(k_{3}\right)}{\left\langle\mu-\Phi_{k_{1}, k_{2}, k_{3}}\right\rangle^{b_{0}}} d \mu
$$

since $\frac{2 b}{q^{\prime}}>b_{0}>\frac{1}{2}$, where

$$
a_{1}\left(k_{1}\right)=\left\|\left\langle\lambda_{1}\right\rangle^{\frac{2 b}{q^{\prime}}} H_{k_{1} k^{*}}\left(\lambda_{1}\right)\right\|_{L_{\lambda_{1}}^{q} l_{k^{*}}^{2}}, \quad a_{j}\left(k_{j}\right)=\left\|\left\langle\lambda_{j}\right\rangle^{b_{0}} \widetilde{v}_{j}\left(\lambda_{j}, k_{j}\right)\right\|_{L_{\lambda_{j}}^{2}}, j=2,3 .
$$

Note that here we used the fact that

$$
\left|\widehat{\chi}\left(\mu-\lambda_{1}+\lambda_{2}-\lambda_{3}-\Phi_{k_{1}, k_{2}, k_{2}}\right)\right| \lesssim\left\langle\mu-\lambda_{1}+\lambda_{2}-\lambda_{3}-\Phi_{k_{1}, k_{2}, k_{2}}\right\rangle^{-10}
$$

and Lemma 2.5. Using Lemma 2.5 again, we have

$$
\int_{|\mu-\lambda| \geq 2|\lambda|} \frac{d \mu}{\langle\mu\rangle\langle\lambda-\mu\rangle^{10}\left\langle\mu-\Phi_{k_{1}, k_{2}, k_{3}}\right\rangle^{b_{0}}} \lesssim \frac{1}{\langle\lambda\rangle\left\langle\lambda-\Phi_{k_{1}, k_{2}, k_{3}}\right\rangle^{b_{0}}} .
$$

By writing

$$
\int_{|\mu-\lambda|<2|\lambda|} \leq \int_{|\mu| \leq \frac{\lambda}{2}}+\int_{|\mu-\lambda| \leq \frac{|\lambda|}{2}}+\int_{|\mu-\lambda| \geq \frac{|\lambda|}{2}, \frac{|\lambda|}{2} \leq|\mu| \leq 3|\lambda|},
$$


we deduce from Lemma 2.5 that

$$
\int_{|\mu-\lambda|<2|\lambda|} \frac{d \mu}{\langle\mu\rangle\langle\lambda-\mu\rangle^{10}\left\langle\mu-\Phi_{k_{1}, k_{2}, k_{3}}\right\rangle^{b_{0}}} \lesssim \epsilon \frac{1}{\langle\lambda\rangle\left\langle\lambda-\Phi_{k_{1}, k_{2}, k_{3}}\right\rangle^{b_{0}-\epsilon}} .
$$

Thus

$$
\int \frac{1}{\langle\mu\rangle}\left(\frac{1}{\langle\lambda\rangle^{10}}+\frac{1}{\langle\lambda-\mu\rangle^{10}}\right) \cdot \frac{1}{\left\langle\mu-\Phi_{k_{1}, k_{2}, k_{3}}\right\rangle^{b_{0}}} d \mu \lesssim \epsilon \frac{1}{\langle\lambda\rangle\left\langle\lambda-\Phi_{k_{1}, k_{2}, k_{3}}\right\rangle^{b_{0}-\epsilon}} .
$$

Next, by Hölder's inequality and Lemma 2.5, we have

$$
\begin{aligned}
& \left\|\langle\lambda\rangle \frac{2 b_{1}}{q^{\prime}} \widetilde{q}_{k k^{*}}(\lambda)\right\|_{L_{\lambda}^{q} l_{k^{*}}^{2}} \lesssim \sum_{\left(k_{1}, k_{2}, k_{3}\right) \in \Gamma(k)} \frac{\prod_{j=1}^{3} a_{j}\left(k_{j}\right)}{\left\langle\Phi_{k_{1}, k_{2}, k_{3}}\right\rangle^{1-\frac{2 b_{1}}{q^{\prime}}}} \\
\lesssim & \sum_{|m| \leq 10 L} \frac{1}{1+\left(m N^{\alpha-1}\right)^{1-\frac{2 b_{1}}{q^{\prime}}}} \sum_{\left(k_{1}, k_{2}, k_{3}\right) \in \Gamma(k)} a_{1}\left(k_{1}\right) a_{2}\left(k_{2}\right) a_{3}\left(k_{3}\right) \mathbf{1}_{\Phi_{k_{1}, k_{2}, k_{3}} \in I_{m}^{(N)}},
\end{aligned}
$$

provided that $q^{\prime}>2 b_{1}$, where $I_{m}^{(N)}=\left[(m-1) N^{\alpha-1}, m N^{\alpha-1}\right]$, since for $N \gg L$, the value of $\Phi_{k_{1}, k_{2}, k_{3}}$ is constraint in $\cup_{|m| \leq 10 L} I_{m}^{(N)}$. Since $\left|\partial_{k_{2}} \Phi_{k_{1}, k_{2}, k_{3}}\right|,\left|\partial_{k_{3}} \Phi_{k_{1}, k_{2}, k_{3}}\right| \gtrsim N^{\alpha-1}$, we have

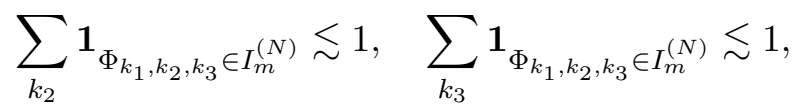

uniformly in $m$. Then by Schur's test, for fixed $k$,

$$
\sum_{k_{2}, k_{3}} a_{1}\left(k+k_{2}-k_{3}\right) a_{2}\left(k_{2}\right) a_{3}\left(k_{3}\right) \mathbf{1}_{\Phi_{k+k_{2}-k_{3}, k_{2}, k_{3}} \in I_{m}^{(N)}} \lesssim\left\|a_{1}\left(k_{1}\right)\right\|_{l_{k_{1}}^{\infty}}\left\|a_{2}\left(k_{2}\right)\right\|_{l_{k_{2}}^{2}}\left\|a_{3}\left(k_{3}\right)\right\|_{l_{k_{3}}^{2}} .
$$

Thus from the elementary inequality

$$
\sum_{|m| \leq 2 L} \frac{1}{1+\left(m N^{\alpha-1}\right)^{\beta}} \leq 1+2 \int_{0}^{10 L} \frac{d z}{1+\left(N^{\alpha-1} z\right)^{\beta}} \lesssim \beta 1+\frac{L^{1-\beta}}{N^{(\alpha-1) \beta}}
$$

for $0<\beta<1$, we have (with $\beta=1-\frac{2 b_{1}}{q^{\prime}}$ )

$$
\begin{aligned}
&\left\|\langle\lambda\rangle^{\frac{2 b_{1}}{q^{\prime}}} \widetilde{q}_{k k^{*}}(\lambda)\right\|_{l_{k}^{\infty} L_{\lambda}^{q} l_{k^{*}}^{2}} \lesssim\left(1+L^{\frac{2 b_{1}}{q^{\prime}}} N^{-(\alpha-1)\left(1-\frac{2 b_{1}}{q^{\prime}}\right)}\right)\|\mathcal{H}\|_{S^{b, q}}\left\|v_{2}\right\|_{X^{0, b_{0}}}\left\|v_{3}\right\|_{X^{0, b_{0}}} \\
& \lesssim\left(L^{-\frac{\alpha-1}{2}+\epsilon_{2}}+L^{\frac{2 b_{1}}{q^{\prime}}-\frac{\alpha-1}{2}+\epsilon_{2}} N^{-(\alpha-1)\left(1-\frac{2 b_{1}}{q^{\prime}}\right)}\right)\|\mathcal{H}\|_{S^{b, q}},
\end{aligned}
$$

since $N_{2} \vee N_{3} \sim L$ and at least (when $v_{2}, v_{3}$ are both of type $(\mathrm{G})$ )

$$
\left\|v_{2}\right\|_{X^{0, b_{0}}} \lesssim N_{2}^{-\frac{\alpha-1}{2}+\epsilon_{2}},\left\|v_{3}\right\|_{X^{0, b_{0}}} \lesssim N_{3}^{-\frac{\alpha-1}{2}+\epsilon_{2}} .
$$

Therefore, whenever

$$
L^{\frac{2 b_{1}}{q^{\prime}}-\frac{\alpha-1}{2}+\epsilon_{2}+\delta_{0}} \lesssim N^{\frac{(\alpha-1)\left(q^{\prime}-2 b_{1}\right)}{q^{\prime}}},
$$

the upper bound $(5.3)$ is $L^{-\delta_{0}}\|\mathcal{H}\|_{S^{b, q}}$, which is conclusive.

-Case 2: $L^{\frac{2 b_{1}}{q^{\prime}}-\frac{\alpha-1}{2}+\epsilon_{2}+\delta_{0}} \gg N^{\frac{(\alpha-1)\left(q^{\prime}-2 b_{1}\right)}{q^{\prime}}}$

In this case, we will reduce to multi-linear sums of low modulations.

By duality, it suffices to estimate

$$
\begin{gathered}
\int \sum_{\begin{array}{c}
k, k_{1}, k_{2}, k_{3}, k^{*} \\
\left(k_{1}, k_{2}, k_{3}\right) \in \Gamma(k)
\end{array}}\langle\lambda\rangle^{\frac{2 b_{1}}{q^{1}}} K(\lambda, \mu) y_{k k^{*}}(\lambda) \widetilde{H}_{k_{1} k^{*}}\left(\lambda_{1}\right) \overline{\widetilde{v}}_{2}\left(\lambda_{2}, k_{2}\right) \widetilde{v}_{3}\left(\lambda_{3}, k_{3}\right) \\
\times \widehat{\chi}\left(\mu-\lambda_{1}+\lambda_{2}-\lambda_{3}-\Phi_{k_{1}, k_{2}, k_{3}}\right) d \lambda_{1} d \lambda_{2} d \lambda_{3} d \mu d \lambda
\end{gathered}
$$


where $\left\|y_{k k^{*}}(\lambda)\right\|_{l_{k}^{1} L_{\lambda}^{q^{\prime}} l_{k^{*}}^{2}}=1$. Summing over $k^{*}$ and using Cauchy-Schwartz, it suffices to estimate the expression

$$
\begin{aligned}
& \sum_{\substack{k, k_{1}, k_{2}, k_{3} \\
\left(k_{1}, k_{2}, k_{3}\right) \in \Gamma(k)}} \int \frac{\langle\lambda\rangle^{\frac{2 b_{1}}{q^{\prime}}}}{\langle\mu\rangle}\left(\frac{1}{\langle\lambda\rangle^{A}}+\frac{1}{\langle\mu-\lambda\rangle^{A}}\right) \cdot \frac{g_{k}(\lambda) f_{k_{1}}\left(\lambda_{1}\right) w_{2}\left(\lambda_{2}, k_{2}\right) w_{3}\left(\lambda_{3}, k_{3}\right)}{\left\langle\lambda_{1}\right\rangle^{\frac{2 b}{q^{\prime}}}\left\langle\lambda_{2}\right\rangle^{b_{0}}\left\langle\lambda_{3}\right\rangle^{b_{0}}} \\
& \times\left|\widehat{\chi}\left(\mu-\lambda_{1}-\lambda_{2}+\lambda_{3}+\Phi_{k_{1}, k_{2}, k_{3}}\right)\right| d \lambda_{1} d \lambda_{2} d \lambda_{3} d \mu d \lambda,
\end{aligned}
$$

where $A \gg 1, g_{k}(\lambda)=\left\|y_{k k^{*}}(\lambda)\right\|_{l_{k^{*}}^{2}}, f_{k_{1}}\left(\lambda_{1}\right)=\left\langle\lambda_{1}\right\rangle^{\frac{2 b}{q^{\prime}}}\left\|\widetilde{H}_{k_{1} k^{*}}\left(\lambda_{1}\right)\right\|_{l_{k^{*}}}$ and $w_{j}\left(\lambda_{j}, k_{j}\right)=$ $\left|\left\langle\lambda_{j}\right\rangle^{b_{0}} \widetilde{v}_{j}\left(\lambda_{j}, k_{j}\right)\right|$ for $j=2,3$. From the rapid decay of $\widehat{\chi}$, the contribution from $\mid \mu-\lambda_{1}+$ $\lambda_{2}-\lambda_{3} \mid \gg N^{\alpha-1} L$ is negligible and can be simply controlled by 7

$$
N^{-10}\left\|g_{k}(\lambda)\right\|_{l_{k}^{1} L_{\lambda}^{q^{\prime}}}\left\|f_{k_{1}}\left(\lambda_{1}\right)\right\|_{l_{k_{1}}^{\infty} L_{\lambda_{1}}^{q}}\left\|w_{2}\left(\lambda_{2}, k_{2}\right)\right\|_{L_{\lambda_{2}}^{2} l_{k_{2}}^{2}}\left\|w_{3}\left(\lambda_{3}, k_{3}\right)\right\|_{L_{\lambda_{3}}^{2} l_{k_{3}}^{2}},
$$

hence we may assume that the multiple integration is taken over $\left|\mu-\lambda_{1}+\lambda_{2}-\lambda_{3}\right| \lesssim N^{\alpha-1} L$. Denote by

$$
\mathcal{T}_{\mu, \lambda, \lambda_{1}, \lambda_{2}, \lambda_{3}}:=\sum_{\substack{k, k_{1}, k_{2}, k_{3} \\\left(k_{1}, k_{2}, k_{3}\right) \in \Gamma(k)}} g_{k}(\lambda) f_{k_{1}}\left(\lambda_{1}\right)\left|\widehat{\chi}\left(\mu-\lambda_{1}+\lambda_{2}-\lambda_{3}-\Phi_{k_{1}, k_{2}, k_{3}}\right)\right| w_{2}\left(\lambda_{2}, k_{2}\right) w_{3}\left(\lambda_{3}, k_{3}\right) .
$$

Note that for fixed $|k| \sim N$ and $\mu_{0} \in \mathbb{R}$,

$$
\sup _{k_{2}} \sum_{k_{3}} f_{k+k_{2}-k_{3}}\left(\lambda_{1}\right) \mathbf{1}_{\left|k_{2}\right|,\left|k_{3}\right| \leq L}\left|\widehat{\chi}\left(\mu_{0}+\Phi_{k+k_{2}-k_{3}, k_{2}, k_{3}}\right)\right| \lesssim\left\|f_{k+k_{2}-k_{3}}\left(\lambda_{1}\right) \mathbf{1}_{\left|k_{2}\right|,\left|k_{3}\right| \leq L}\right\|_{l_{k_{2}, k_{3}}^{\infty}},
$$

and

$$
\sup _{k_{3}} \sum_{k_{2}} f_{k+k_{2}-k_{3}}\left(\lambda_{1}\right) \mathbf{1}_{\left|k_{2}\right|,\left|k_{3}\right| \leq L}\left|\widehat{\chi}\left(\mu_{0}+\Phi_{k+k_{2}-k_{3}, k_{2}, k_{3}}\right)\right| \lesssim\left\|f_{k+k_{2}-k_{3}}\left(\lambda_{1}\right) \mathbf{1}_{\left|k_{2}\right|,\left|k_{3}\right| \leq L}\right\|_{l_{k_{2}, k_{3}}^{\infty}}
$$

since $\left|\partial_{k_{j}} \Phi_{k+k_{2}-k_{3}, k_{2}, k_{3}}\right| \gtrsim N^{\alpha-1} \gg 1$ for $j=2,3$. Applying Schur's test, we have

$$
\begin{aligned}
& \sum_{\substack{k_{2}, k_{3} \\
\left|k_{j}\right| \sim N_{j}, j=2,3}} f_{k+k_{2}-k_{3}}\left(\lambda_{1}\right)\left|\widehat{\chi}\left(\mu_{0}+\Phi_{k+k_{2}-k_{3}, k_{2}, k_{3}}\right)\right| w_{2}\left(\lambda_{2}, k_{2}\right) w_{3}\left(\lambda_{3}, k_{3}\right) \\
\lesssim & \left\|f_{k+k_{2}-k_{3}}\left(\lambda_{1}\right) \mathbf{1}_{\left|k_{2}\right| \leq L,\left|k_{3}\right| \leq L}\right\|_{l_{k_{2}, k_{3}}^{\infty}}\left\|w_{2}\left(\lambda_{2}, k_{2}\right)\right\|_{l_{k_{2}}^{2}}\left\|w_{3}\left(\lambda_{3}, k_{3}\right)\right\|_{l_{k_{3}}^{2}} \\
\leq & \left\|f_{k+k_{2}-k_{3}}\left(\lambda_{1}\right) \mathbf{1}_{\left|k_{2}\right| \leq L,\left|k_{3}\right| \leq L}\right\|_{l_{k_{2}, k_{3}}^{q}}\left\|w_{2}\left(\lambda_{2}, k_{2}\right)\right\|_{l_{k_{2}}^{2}}\left\|w_{3}\left(\lambda_{3}, k_{3}\right)\right\|_{l_{k_{3}}^{2}},
\end{aligned}
$$

where to the last inequality, we use the embedding $l^{q} \rightarrow l^{\infty}$. Writing $\varphi_{j}(\lambda)=\left\|w_{j}\left(\lambda_{j}, k_{j}\right)\right\|_{l_{k_{j}}^{2}}$ for $j=2,3$, by Hölder we estimate the contribution from $\frac{1}{\langle\mu\rangle\langle\lambda\rangle^{A}}$ in 5.4 by

$$
\begin{aligned}
& \int \mathbf{1}_{\left|\mu-\lambda_{1}+\lambda_{2}-\lambda_{3}\right| \lesssim N^{\alpha-1} L}\langle\mu\rangle^{-1}\langle\lambda\rangle^{-A+\frac{2 b_{1}}{q^{\prime}}}\left\langle\lambda_{1}\right\rangle^{-\frac{2 b}{q^{\prime}}}\left\langle\lambda_{2}\right\rangle^{-b_{0}}\left\langle\lambda_{3}\right\rangle^{-b_{0}} \mathcal{T}_{\mu, \lambda, \lambda_{1}, \lambda_{2}, \lambda_{3}} d \mu d \lambda d \lambda_{1} d \lambda_{2} d \lambda_{3} \\
& \lesssim \epsilon N^{\epsilon} \sum_{k} \int g_{k}(\lambda)\langle\lambda\rangle^{-A+\frac{2 b_{1}}{q^{\prime}}}\left\langle\lambda_{1}\right\rangle^{-\frac{2 b}{q^{\prime}}}\left\langle\lambda_{2}\right\rangle^{-b_{0}}\left\langle\lambda_{3}\right\rangle^{-b_{0}} \varphi_{2}\left(\lambda_{2}\right) \varphi_{3}\left(\lambda_{3}\right)\left\|f_{k+k_{2}-k_{3}}\left(\lambda_{1}\right) \mathbf{1}_{\left|k_{2}\right| \leq L}\right\|_{k_{3} \mid \leq L} \|_{l_{k_{2}, k_{3}}^{q}} d \lambda d \lambda_{1} d \lambda_{2} d \lambda_{3} \\
& \lesssim N^{\epsilon} \sum_{k}\left\|g_{k}(\lambda)\right\|_{L_{\lambda}^{q^{\prime}}}\left\|\varphi_{2}\left(\lambda_{2}\right)\right\|_{L_{\lambda_{2}}^{2}}\left\|\varphi_{3}\left(\lambda_{3}\right)\right\|_{L_{\lambda_{3}}^{2}}\left\|f_{k+k_{2}-k_{3}}\left(\lambda_{1}\right) \mathbf{1}_{\left|k_{2}\right| \leq L}\right\|_{k_{3} \mid \leq L} \|_{l_{k_{2}, k_{3}}^{q} L_{\lambda_{1}}^{q}} \\
& \lesssim N^{\epsilon}\left\|g_{k}(\lambda)\right\|_{l_{k}^{1} L_{\lambda}^{q^{\prime}}}\left\|\varphi_{2}\left(\lambda_{2}\right)\right\|_{L_{\lambda_{2}}^{2}}\left\|\varphi_{3}\left(\lambda_{3}\right)\right\|_{L_{\lambda_{3}}^{2}}\left\|f_{k+k_{2}-k_{3}}\left(\lambda_{1}\right) \mathbf{1}_{\left|k_{2}\right| \leq L}\right\|_{k_{3} \mid \leq L} \|_{l_{k}^{\infty} l_{k_{2}, k_{3}}^{q} L_{\lambda_{1}}^{q}} \\
& \lesssim N^{\epsilon} L^{\frac{2}{q}}\left\|g_{k}(\lambda)\right\|_{l_{k}^{1} L_{\lambda}^{q^{\prime}}}\left\|\varphi_{2}\left(\lambda_{2}\right)\right\|_{L_{\lambda_{2}}^{2}}\left\|\varphi_{3}\left(\lambda_{3}\right)\right\|_{L_{\lambda_{3}}^{2}}\left\|f_{k_{1}}\left(\lambda_{1}\right)\right\|_{l_{k}^{\infty} L_{\lambda_{1}}^{q}} .
\end{aligned}
$$

It remains to estimate the last contribution from $\frac{1}{\langle\mu\rangle\langle\mu-\lambda\rangle^{A}}$ in 5.4 . Note that the integration over $|\lambda-\mu| \gg N^{\alpha-1} L$ gives us an error like (5.5), hence we can assume that

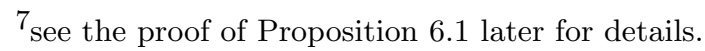


$|\lambda-\mu| \lesssim N^{\alpha-1} L$, and in particular, $\left|\lambda-\lambda_{1}+\lambda_{2}-\lambda_{3}\right| \lesssim N^{\alpha-1} L$, since the region of integration is $\left|\mu-\lambda_{1}+\lambda_{2}-\lambda_{3}\right| \lesssim N^{\alpha-1} L$. From Lemma 2.5

$$
\int \frac{\langle\lambda\rangle^{\frac{2 b_{1}}{q^{\prime}}}}{\langle\mu\rangle\langle\mu-\lambda\rangle^{A}} d \mu \lesssim\langle\lambda\rangle^{-1+\frac{2 b_{1}}{q^{\prime}}} .
$$

Using (5.6), the inequality above, and then Hölder's inequality for the integration in $\lambda$, we have

$$
\begin{aligned}
& \int \mathbf{1}_{\left|\mu-\lambda_{1}+\lambda_{2}-\lambda_{3}\right| \lesssim N^{\alpha-1} L}\langle\mu\rangle^{-1}\langle\lambda-\mu\rangle^{-A}\langle\lambda\rangle^{\frac{2 b_{1}}{q^{\prime}}}\left\langle\lambda_{1}\right\rangle^{-\frac{2 b}{q^{\prime}}}\left\langle\lambda_{2}\right\rangle^{-b_{0}}\left\langle\lambda_{3}\right\rangle^{-b_{0}} \mathcal{T}_{\mu, \lambda, \lambda_{1}, \lambda_{2}, \lambda_{3}-\lambda_{3} \mid \lesssim N^{\alpha-1} L} d \mu d \lambda d \lambda_{1} d \lambda_{2} d \lambda_{3} \\
\lesssim & \sum_{k} \int \mathbf{1}_{\left|\lambda-\lambda_{1}+\lambda_{2}-\lambda_{3}\right| \lesssim N^{\alpha-1} L} \cdot g_{k}(\lambda)\langle\lambda\rangle^{-\frac{q^{\prime}-2 b_{1}}{q^{\prime}}}\left\langle\lambda_{1}\right\rangle^{-\frac{2 b}{q^{\prime}}}\left\langle\lambda_{2}\right\rangle^{-b_{0}}\left\langle\lambda_{3}\right\rangle^{-b_{0}} \varphi_{2}\left(\lambda_{2}\right) \varphi_{3}\left(\lambda_{3}\right) \\
\times & \left\|f_{k+k_{2}-k_{3}}\left(\lambda_{1}\right) \mathbf{1}_{\left|k_{2}\right| \leq L,\left|k_{3}\right| \leq L}\right\|_{l_{k_{2}, k_{3}}^{q}} d \lambda d \lambda_{1} d \lambda_{2} d \lambda_{3} \\
\lesssim & \sum_{k}\left\|g_{k}(\lambda)\right\|_{L_{\lambda}^{q^{\prime}}}\left\|\mathbf{1}_{|\lambda-\cdot| \lesssim N^{\alpha-1} L}\right\| \frac{q^{\prime}}{L_{\lambda}^{2 b_{1}-1+\epsilon}}\left\|\langle\lambda\rangle^{-\frac{q^{\prime}-2 b_{1}}{q^{\prime}}}\right\|{ }_{L_{\lambda}^{q^{\prime}-2 b_{1}-\epsilon}} \\
\times & \int\left\langle\lambda_{1}\right\rangle^{-\frac{2 b}{q^{\prime}}}\left\langle\lambda_{2}\right\rangle^{-b_{0}}\left\langle\lambda_{3}\right\rangle^{-b_{0}} \varphi_{2}\left(\lambda_{2}\right) \varphi_{3}\left(\lambda_{3}\right)\left\|f_{k+k_{2}-k_{3}}\left(\lambda_{1}\right) \mathbf{1}_{\left|k_{2}\right| \leq L,\left|k_{3}\right| \leq L}\right\|_{l_{k_{2}, k_{3}}} d \lambda_{1} d \lambda_{2} d \lambda_{3},
\end{aligned}
$$

for $\epsilon>0$ small and to be chosen later. Next we use Hölder's inequality for the integration in $\lambda_{1}, \lambda_{2}, \lambda_{3}$, the above quantity can be bounded by

$$
\begin{aligned}
& N^{\frac{(\alpha-1)\left(2 b_{1}-1+\epsilon\right)}{q^{\prime}}} L^{\frac{2 b_{1}-1+\epsilon}{q^{\prime}}} \sum_{k}\left\|g_{k}(\lambda)\right\|_{L_{\lambda}^{q^{\prime}}}\left\|\varphi_{2}\left(\lambda_{2}\right)\right\|_{L_{\lambda_{2}}^{2}}\left\|\varphi_{3}\left(\lambda_{3}\right)\right\|_{L_{\lambda_{3}}^{2}}\left\|f_{k+k_{2}-k_{3}}\left(\lambda_{1}\right) \mathbf{1}_{\left|k_{2}\right| \leq L}\right\|_{k_{3} \mid \leq L} \|_{l_{k_{2}, k_{3}}^{q} L_{\lambda_{1}}^{q}} \\
\leq & N^{\frac{(\alpha-1)\left(2 b_{1}-1+\epsilon\right)}{q^{\prime}}} L^{\frac{2 b_{1}-1+\epsilon}{q^{\prime}}+\frac{2}{q}}\left\|g_{k}(\lambda)\right\|_{l_{k}^{1} L_{\lambda}^{q^{\prime}}}\left\|\varphi_{2}\left(\lambda_{2}\right)\right\|_{L_{\lambda_{2}}^{2}}\left\|\varphi_{3}\left(\lambda_{3}\right)\right\|_{L_{\lambda_{3}}^{2}}\left\|f_{k_{1}}\left(\lambda_{1}\right)\right\|_{l_{k}^{\infty} L_{\lambda_{1}}^{q}} .
\end{aligned}
$$

Since

$$
\left\|\varphi_{j}\left(\lambda_{j}\right)\right\|_{L_{\lambda_{j}}^{2}}=\left\|v_{j}\right\|_{X^{0, b_{0}}} \lesssim N_{j}^{-\frac{\alpha-1}{2}+\epsilon_{2}}, j=2,3
$$

and $N_{2} \vee N_{3} \sim L$, we finally have (fixing $\epsilon=\epsilon_{2}$, say)

$$
\left\|\langle\lambda\rangle^{\frac{2 b_{1}}{q^{\prime}}} \widetilde{q}_{k k^{*}}(\lambda)\right\|_{l_{k}^{\infty} L_{\lambda}^{q} l_{k^{*}}^{2}} \lesssim N^{\frac{(\alpha-1)\left(2 b_{1}-1+\epsilon\right)}{q^{\prime}}} L^{-\frac{\alpha-1}{2}+\frac{2 b_{1}-1+\epsilon_{2}}{q^{\prime}}+\frac{2}{q}+\epsilon_{2}+\delta_{0}} \cdot L^{-\delta_{0}}\|\mathcal{H}\|_{S^{b, q}} .
$$

Recall that for the case 2 ,

$$
L^{\frac{2 b_{1}}{q^{\prime}}-\frac{\alpha-1}{2}+\epsilon_{2}+\delta_{0}} \gg N^{\frac{(\alpha-1)\left(q^{\prime}-2 b_{1}\right)}{q^{\prime}}},
$$

then

$$
L^{\frac{\alpha-1}{2}-\frac{2 b_{1}-1}{q^{\prime}}-\frac{2}{q}-\epsilon_{2}-\delta_{0}} \gg N^{\frac{(\alpha-1)\left(q^{\prime}-2 b_{1}\right)}{q^{\prime}} \cdot\left(\frac{2 b_{1}}{q^{\prime}}-\frac{\alpha-1}{2}+\epsilon_{2}+\delta_{0}\right)^{-1}} .
$$

By our definition of numerical parameters in (3.1), if the free parameter $\sigma$ is chosen small enough, we have $\frac{(\alpha-1)\left(q^{\prime}-2 b_{1}\right)}{q^{\prime}} \cdot\left(\frac{2 b_{1}}{q^{\prime}}-\frac{\alpha-1}{2}+\epsilon_{2}+\delta_{0}\right)^{-1} \geq \frac{(\alpha-1)\left(2 b_{1}-1\right)}{q^{\prime}}$, in particular,

$$
L^{\frac{\alpha-1}{2}-\frac{2 b_{1}-1+\epsilon}{q^{\prime}}-\frac{2}{q}-\epsilon_{2}-\delta_{0}} \geq N^{\frac{(\alpha-1)\left(2 b_{1}-1+\epsilon_{2}\right)}{q^{\prime}}} .
$$

This completes the proof of (6) of Proposition 3.7 .

5.2. $X^{0, b}$-mapping property of the operator norm of $\mathcal{P}_{L}^{ \pm}$. In this subsection, we prove (3),(5),(7) of Proposition 3.7. The key point is the following lemma:

Lemma 5.1. Assume that $v_{1}, v_{2}, v_{3}$ are of type $(G),(C)$ or $(D)$ with characterized parameters $\left(N_{j}, L_{j}\right), j=1,2,3$, then

$$
\left\|\mathcal{N}_{3}\left(v, v_{2}, v_{3}\right)\right\|_{X^{0, b_{1}-1}} \lesssim\left(N_{2} \vee N_{3}\right)^{-\delta_{0}}\|v\|_{X^{0, \frac{3}{8}}}
$$

and

$$
\left\|\mathcal{N}_{3}\left(v_{1}, v, v_{3}\right)\right\|_{X^{0, b_{1}-1}} \lesssim\left(N_{1} \vee N_{3}\right)^{-\delta_{0}}\|v\|_{X^{0, \frac{3}{8}}}
$$


Moreover, the same estimate holds, with uniform implicit constants on the r.h.s., if we replace $v_{1}, v_{2}, v_{3}$ by $\Pi_{M_{1}} v_{1}, \Pi_{M_{2}} v_{2}$ and $\Pi_{M_{3}} v_{3}$ for any dyadic numbers $M_{1}, M_{2}, M_{3}$.

Proof. We will only prove the estimate for $\mathcal{N}_{3}\left(v, v_{2}, v_{3}\right)$, since the other follows from the same argument. By duality, it suffices to show that ${ }^{8}$, for every $w \in X^{0,1-b_{1}},\|w\|_{X^{0,1-b_{1}}} \leq 1$, we have

$$
\left|\iint v \bar{v}_{2} v_{3} \cdot \bar{w} d t d x\right| \lesssim\left(N_{2} \vee N_{3}\right)^{-\delta_{0}}\|v\|_{X^{0, \frac{3}{8}}} \cdot
$$

Splitting the functions as Littlewood-Paley pieces, $v_{j}=\sum_{M_{j}} \mathbf{P}_{M_{j}} v_{j}, j=2,3$ and $v=$ $\sum_{M_{1}} \mathbf{P}_{M_{1}} v, w=\sum_{M} \mathbf{P}_{M} w$.

-Case 1: $v_{2}, v_{3}$ are both of type (D)

In this case, we have

$$
\left\|\mathbf{P}_{M_{j}} v_{j}\right\|_{X^{0, b}} \lesssim\left(M_{j} \vee N_{j}\right)^{-s}, \quad j=2,3 .
$$

By inserting $\chi(t)$ into $w$ and using the bilinear Strichartz (Lemma 2.10), we have

$$
\begin{aligned}
& \left|\iint v \bar{v}_{2} v_{3} \cdot \bar{w} d t d x\right| \\
\lesssim & \sum_{\substack{M_{1}, M_{2}, M_{3}, M \\
M \lesssim M_{1} \vee M_{2} \vee M_{3}}}\left(M_{(2)} M_{(3)}\right)^{\frac{1}{2}-\frac{\alpha}{4}}\left\|\mathbf{P}_{M_{1}} v\right\|_{X^{0, \frac{3}{8}}}\left\|\mathbf{P}_{M_{2}} v_{2}\right\|_{X^{0, \frac{3}{8}}}\left\|\mathbf{P}_{M_{3}} v_{3}\right\|_{X^{0, \frac{3}{8}}}\left\|\mathbf{P}_{M^{\prime} w}\right\|_{X^{0, \frac{3}{8}}},
\end{aligned}
$$

where $M_{(1)} \geq M_{(2)} \geq M_{(3)}$ is the non-increasing re-ordering of $M_{1}, M_{2}, M_{3}$. Without loss of generality, we may assume that $M_{2} \geq M_{3}$. Note that when $M_{1} \gg M_{2} \vee M_{3}$, we must have $M \sim M_{1}$ in the sum, otherwise $\int \mathbf{P}_{M_{1}} v \cdot \mathbf{P}_{M_{2}} \bar{v}_{2} \mathbf{P}_{M_{3}} v \cdot \mathbf{P}_{M} \bar{w} d x=0$. We estimate this contribution as

$$
\begin{aligned}
& \sum_{\substack{M, M_{1}, M_{2}, M_{3} \\
M \sim M_{1} \gg M_{2} \geq M_{3}}}\left(M_{(2)} M_{(3)}\right)^{\frac{1}{2}-\frac{\alpha}{4}}\left\|\mathbf{P}_{M_{1}} v\right\|_{X^{0, \frac{3}{8}}}\left\|\mathbf{P}_{M_{2}} v_{2}\right\|_{X^{0, \frac{3}{8}}}\left\|\mathbf{P}_{M_{3}} v_{3}\right\|_{X^{0, \frac{3}{8}}}\left\|\mathbf{P}_{M} w\right\|_{X^{0, \frac{3}{8}}} \\
\lesssim & \sum_{\substack{M, M_{1} \\
M \sim M_{1}}}\left\|\mathbf{P}_{M_{1}} v\right\|_{X^{0, \frac{3}{8}}}\left\|\mathbf{P}_{M} w\right\|_{X^{0, \frac{3}{8}}} \sum_{\substack{M_{2}, M_{3} \\
M_{3} \leq M_{2} \ll M_{1}}}\left(M_{2} M_{3}\right)^{\frac{1}{2}-\frac{\alpha}{4}}\left(N_{2} \vee M_{2}\right)^{-s}\left(N_{3} \vee M_{3}\right)^{-s} \\
\lesssim & \left(N_{2} \vee N_{3}\right)^{\frac{1}{2}-\frac{\alpha}{4}-s}\|v\|_{X^{0, \frac{3}{8}}},
\end{aligned}
$$

where to the final inequality, we use the fact that $s-\left(\frac{1}{2}-\frac{\alpha}{4}\right)=\sigma>0, \min \left\{b, 1-b_{1}\right\}>\frac{3}{8}$ and Cauchy-Schwartz for the sum $\sum_{M \sim M_{1}}$. For the contribution of $M_{1} \lesssim M_{2}$, we estimate it as

$$
\begin{aligned}
& \sum_{\substack{M, M_{1}, M_{2}, M_{3} \\
M_{3} \leq M_{2} \\
M, M_{1} \lesssim M_{2}}}\left(M_{(2)} M_{(3)}\right)^{\frac{1}{2}-\frac{\alpha}{4}}\left\|\mathbf{P}_{M_{1}} v\right\|_{X^{0, \frac{3}{8}}}\left\|\mathbf{P}_{M_{2}} v_{2}\right\|_{X^{0, \frac{3}{8}}}\left\|\mathbf{P}_{M_{3}} v_{3}\right\|_{X^{0, \frac{3}{8}}}\left\|\mathbf{P}_{M^{\prime}} w\right\|_{X^{0, \frac{3}{8}}} \\
& \lesssim \sum_{\substack{M, M_{1}, M_{2}, M_{3} \\
M_{3} \leq M_{2} \\
M, M_{1} \lesssim M_{2}}}\left(M_{2} M_{3}\right)^{\frac{1}{2}-\frac{\alpha}{4}}\left(M_{2} \vee N_{2}\right)^{-s}\left(N_{3} \vee M_{3}\right)^{-s}\left\|\mathbf{P}_{M_{1}} v\right\|_{X^{0, \frac{3}{8}}}\left\|\mathbf{P}_{M} w\right\|_{X^{0, \frac{3}{8}}} \\
& \lesssim \sum_{M_{2}, M_{3}: M_{3} \leq M_{2}}\left(M_{2} M_{3}\right)^{\frac{1}{2}-\frac{\alpha}{4}} \log \left(M_{2}\right)^{2}\left(M_{2} \vee N_{2}\right)^{-s}\left(M_{3} \vee N_{3}\right)^{-s} \cdot\|v\|_{X^{0, \frac{3}{8}}} \\
& \lesssim \epsilon\left(N_{2} \vee N_{3}\right)^{\frac{1}{2}-\frac{\alpha}{4}-s+\epsilon}\|v\|_{X^{0, \frac{3}{8}}} \leq\left(N_{2} \vee N_{3}\right)^{-\delta_{0}}\|v\|_{X^{0, \frac{3}{8}}},
\end{aligned}
$$

by choosing $0<\epsilon<\sigma-\delta_{0}$ (which is positive if $\sigma \ll 1$ ) here. Therefore,

$$
\left|\iint v \bar{v}_{2} v_{3} \cdot \bar{w} d x d t\right| \lesssim\left(N_{2} \vee N_{3}\right)^{-\delta_{0}}\|v\|_{X^{0,3}} \cdot
$$

- Case 2: One of $v_{2}, v_{3}$ is of type (D) and the other is of type (G) or (C)

\footnotetext{
${ }^{8}$ We omit the estimate for the diagonal nonlinearities $\mathcal{N}_{0}\left(v, v_{2}, v_{3}\right)$ here, since this term is always better and will be treated in Section 9
} 
Without loss of generality, we may assume that $v_{2}$ is of type (D) and $v_{3}$ is of type $(\mathrm{G})$ or $(\mathrm{C})$, thus

$$
\left\|\mathbf{P}_{M_{2}} v_{2}\right\|_{X^{0, b}} \lesssim\left(M_{2} \vee N_{2}\right)^{-s}
$$

Using the bilinear Strichartz inequality, we have

$$
\begin{aligned}
& \quad\left|\iint \mathbf{P}_{M_{1}} v \mathbf{P}_{M_{2}} \bar{v}_{2} v_{3} \cdot \mathbf{P}_{M} \bar{w} d x d t\right| \leq\left\|\mathbf{P}_{M_{1}} v \mathbf{P}_{M_{2}} v_{2}\right\|_{L_{t, x}^{2}}\left\|v_{3}\right\|_{L_{t}^{4} L_{x}^{\infty}}\left\|\mathbf{P}_{M} w\right\|_{L_{t}^{4} L_{x}^{2}} \\
& \lesssim\left(M_{1} \wedge M_{2}\right)^{\frac{1}{2}-\frac{\alpha}{4}}\left\|\mathbf{P}_{M_{1}} v\right\|_{X^{0, \frac{3}{8}}}\left\|\mathbf{P}_{M_{2}} v_{2}\right\|_{X^{0, \frac{3}{8}}}\left\|\mathbf{P}_{M} w\right\|_{X^{0, \frac{1}{4}}}\left\|v_{3}\right\|_{L_{t}^{4} L_{x}^{\infty} .}
\end{aligned}
$$

We then take the dyadic summation in $M_{1}, M_{2}$ and $M$. Since the Fourier support of $v_{3}$ is constraint at $\left|k_{3}\right| \lesssim N_{3}$, the contribution for $M \vee M_{1} \vee M_{2} \lesssim N_{3}$ is bounded by

$$
\sum_{\substack{M, M_{1}, M_{2} \\ M \vee M_{1} \vee M_{2} \lesssim N_{3}}}\left(M_{1} \wedge M_{2}\right)^{\frac{1}{2}-\frac{\alpha}{4}}\left\|\mathbf{P}_{M_{1}} v\right\|_{X^{0, \frac{3}{8}}}\left\|\mathbf{P}_{M} w\right\|_{X^{0, \frac{1}{4}}}\left\|\mathbf{P}_{M_{2}} v_{2}\right\|_{X^{0, \frac{3}{8}}} \lesssim N_{2}^{\frac{1}{2}-\frac{\alpha}{4}-s} \log \left(N_{3}\right)^{2}\|v\|_{X^{0, \frac{3}{8}}} \text {. }
$$

When $M \vee M_{1} \vee M_{2} \gg N_{3}$, then one of the situations must happen: $M \sim M_{1} \sim M_{2}$, or $M \sim M_{1} \gg M_{2}$, or $M \sim M_{2} \gg M_{1}$, or $M_{1} \sim M_{2} \gg M$. Therefore, we have

$$
\sum_{\substack{M, M_{1}, M_{2} \\ M \vee M_{1} \vee M_{2} \gg N_{3}}}\left(M_{1} \wedge M_{2}\right)^{\frac{1}{2}-\frac{\alpha}{4}}\left\|\mathbf{P}_{M_{1}} v\right\|_{X^{0, \frac{1}{4}}}\left\|\mathbf{P}_{M} w\right\|_{X^{0, \frac{1}{4}}}\left\|\mathbf{P}_{M_{2}} v_{2}\right\|_{X^{0, \frac{3}{8}}} \lesssim \epsilon N_{2}^{\frac{1}{2}-\frac{\alpha}{4}-s+\epsilon}\|v\|_{X^{0, \frac{3}{8}}} .
$$

Choosing $0<\epsilon<\delta_{0}-\delta$, we obtain that

$$
\left|\iint v \bar{v}_{2} v_{3} \cdot \bar{w} d x d t\right| \lesssim N_{2}^{-\delta_{0}}\left\|v_{3}\right\|_{L_{t}^{4} L_{x}^{\infty}}\|v\|_{X^{0, \frac{3}{8}}}
$$

Alternatively, we use the bilinear Strichartz inequality as for the Case 1 and obtain that

$$
\sum_{M_{1}, M_{2}, M, M_{3} \lesssim N_{3}}\left|\iint \mathbf{P}_{M_{1}} v \mathbf{P}_{M_{2}} \bar{v}_{2} \mathbf{P}_{M_{3}} v \cdot \mathbf{P}_{M} \bar{w} d x d t\right| \lesssim N_{2}^{-\delta_{0}} N_{3}^{\frac{1}{2}-\frac{\alpha}{4}}\left\|v_{3}\right\|_{X^{0, \frac{3}{8}}}\|v\|_{X^{0, \frac{3}{8}}} \cdot
$$

Therefore, we have

$$
\left|\iint v \bar{v}_{2} v_{3} \cdot \bar{w} d x d t\right| \lesssim N_{2}^{-\delta_{0}}\|v\|_{X^{0, \frac{3}{8}}} \min \left\{\left\|v_{3}\right\|_{L_{t}^{4} L_{x}^{\infty}}, N_{3}^{\frac{1}{2}-\frac{\alpha}{4}}\left\|v_{3}\right\|_{X^{0, \frac{3}{8}}}\right\} .
$$

When $v_{3}$ is of type $(\mathrm{G})$, then $\left\|v_{3}\right\|_{L_{t}^{4} L_{x}^{\infty}}<N_{3}^{-\frac{\alpha-1}{2}+\epsilon_{2}}$ which is conclusive. When $v_{3}$ is of type $(\mathrm{C})$, we have

$$
\begin{aligned}
\min \left\{\left\|v_{3}\right\|_{L_{t}^{4} L_{x}^{\infty}}, N_{3}^{\frac{1}{2}-\frac{\alpha}{4}}\left\|v_{3}\right\|_{X^{0, \frac{3}{8}}}\right\} & \lesssim \min \left\{N_{3}^{-(\alpha-1)+\epsilon_{2}} L_{3}^{\frac{1}{2}-\nu}, N_{3}^{\frac{1}{2}-\frac{\alpha}{4}-(\alpha-1)+\epsilon_{2}} L_{3}^{-\nu}\right\} \\
& \lesssim N_{3}^{-(\alpha-1)+s-2 s \nu+\epsilon_{2}-(1+2 \nu) \sigma}
\end{aligned}
$$

which is conclusive since $\epsilon_{2}<(1+2 \nu) \sigma$ and

$$
(\alpha-1)+2 s \nu>s,
$$

thanks to the choice of numerical parameters.

-Case 3: $v_{2}, v_{3}$ are both of type $(\mathbf{G})$ or $(\mathbf{C})$

In this case, $\mathbf{P}_{M_{j}} v_{j}=0$ when $M_{j} \gg N_{j}$. Therefore, by splitting as

$$
\iint v \bar{v}_{2} v_{3} \bar{w} d t d x=\sum_{M_{1}, M} \iint \mathbf{P}_{M_{1}} v \bar{v}_{2} v_{3} \mathbf{P}_{M} \bar{w} d t d x
$$

we may assume that either $M \sim M_{1} \gg N_{2} \vee N_{3}$ or $M, M_{1} \lesssim N_{2} \vee N_{3}$. By Hölder,

$$
\begin{aligned}
& \quad\left|\iint \mathbf{P}_{M_{1}} v \bar{v}_{2} v_{3} \cdot \mathbf{P}_{M} \bar{w} d x d t\right| \leq\left\|\mathbf{P}_{M_{1}} v\right\|_{L_{t}^{4} L_{x}^{2}}\left\|\mathbf{P}_{M} w\right\|_{L_{t}^{4} L_{x}^{2}}\left\|v_{2}\right\| L_{L_{t}^{4} L_{x}^{\infty}}\left\|v_{3}\right\|_{L_{t}^{4} L_{x}^{\infty}} \\
& \lesssim\left\|\mathbf{P}_{M_{1}} v\right\|_{X^{0, \frac{1}{4}}}\left\|\mathbf{P}_{M} w\right\|_{X^{0, \frac{1}{4}}}\left\|v_{2}\right\|_{L_{t}^{4} L_{x}^{\infty}}\left\|v_{3}\right\|_{L_{t}^{4} L_{x}^{\infty}} .
\end{aligned}
$$


Therefore, we have

$$
\sum_{\substack{M_{1}, M \\ M_{1} \sim M \gg N_{2} \vee N_{3}}}\left\|\mathbf{P}_{M_{1}} v\right\|_{X^{0, \frac{1}{4}}}\left\|\mathbf{P}_{M} w\right\|_{X^{0, \frac{1}{8}}}\left\|v_{2}\right\|_{L_{t}^{4} L_{x}^{\infty}}\left\|v_{3}\right\|_{L_{t}^{4} L_{x}^{\infty}} \lesssim\|v\|_{X^{0, \frac{1}{4}}}\left\|v_{2}\right\|_{L_{t}^{4} L_{x}^{\infty}}\left\|v_{3}\right\|_{L_{t}^{4} L_{x}^{\infty}},
$$

and

$\sum_{M_{1}, M \lesssim N_{*}}\left\|\mathbf{P}_{M_{1}} v\right\|_{X^{0, \frac{1}{4}}}\left\|\mathbf{P}_{M} v\right\|_{X^{0, \frac{1}{8}}}\left\|v_{2}\right\|_{L_{t}^{4} L_{x}^{\infty}}\left\|v_{3}\right\|_{L_{t}^{4} L_{x}^{\infty}} \lesssim \log \left(N_{2} \vee N_{3}\right)^{2}\|v\|_{X^{0, \frac{1}{4}}}\left\|v_{2}\right\|_{L_{t}^{4} L_{x}^{\infty}}\left\|v_{3}\right\|_{L_{t}^{4} L_{x}^{\infty}}$.

Alternatively, using the bilinear Strichartz, we have

$$
\begin{aligned}
& \sum_{\substack{M, M_{1}, M_{2}, M \\
\vdots}}\left|\iint \mathbf{P}_{M_{1}} v \mathbf{P}_{M_{2}} \bar{v}_{2} \mathbf{P}_{M_{2}} v_{3} \mathbf{P}_{M} \bar{w} d t d x\right|_{\substack{M, M_{1}, M_{2}, M_{3} \\
M_{2} \leq N_{2}, M_{3} \leq N_{3} \\
M \sim M_{1} \gg N_{2} \vee N_{3}}}\left\|\mathbf{P}_{M_{1}} v\right\|_{X^{0, \frac{3}{8}}}\left\|\mathbf{P}_{M} w\right\|_{X^{0, \frac{3}{8}}}\left(M_{2} M_{3}\right)^{\frac{1}{2}-\frac{\alpha}{4}}\left\|\mathbf{P}_{M_{2}} v_{2}\right\|_{X^{0, \frac{3}{8}}}\left\|\mathbf{P}_{M_{3}} v_{3}\right\|_{X^{0, \frac{3}{8}}} \\
&+ \sum_{\substack{M, M_{1}, M_{2}, M_{3} \\
M_{2} \leq N_{2}, M_{3} \leq N_{3} \\
M, M_{1} \lesssim N_{2} \vee N_{3}}}\left(M_{2} M_{3}\right)^{\frac{1}{2}-\frac{\alpha}{4}}\left\|\mathbf{P}_{M_{1}} v\right\|_{X^{0, \frac{3}{8}}}\left\|\mathbf{P}_{M_{2}} v_{2}\right\|_{X^{0, \frac{3}{8}}}\left\|\mathbf{P}_{M_{1}} v_{3}\right\|_{X^{0, \frac{3}{8}}}\left\|\mathbf{P}_{M w} w\right\|_{X^{0, \frac{3}{8}}} \\
& \lesssim\left(N_{2} N_{3}\right)^{\frac{1}{2}-\frac{\alpha}{4}}\left(\log \left(N_{2} \vee N_{3}\right)\right)^{4}\|v\|_{X^{0, \frac{3}{8}}}\left\|v_{2}\right\|_{X^{0, \frac{3}{8}}}\left\|v_{3}\right\|_{X^{0, \frac{3}{8}}} \cdot
\end{aligned}
$$

Thus we have

$$
\begin{aligned}
& \quad\left|\iint v \bar{v}_{2} v_{3} \cdot \bar{w} d x d t\right| \\
& \lesssim_{\epsilon}\|v\|_{X^{0, \frac{3}{8}}}\left(N_{2} \vee N_{3}\right)^{\epsilon} \min \left\{\left\|v_{2}\right\|_{L_{t}^{4} L_{x}^{\infty}}\left\|v_{3}\right\|_{L_{t}^{4} L_{x}^{\infty}},\left(N_{2} N_{3}\right)^{\frac{1}{2}-\frac{\alpha}{4}}\left\|v_{2}\right\|_{X^{0, \frac{3}{8}}}\left\|v_{3}\right\|_{X^{0, \frac{3}{8}}}\right\} .
\end{aligned}
$$

When $v_{2}, v_{3}$ are both of type $(\mathrm{G})$, the bound $\left\|v_{2}\right\|_{L_{t}^{4} L_{x}^{\infty}}\left\|v_{3}\right\|_{L_{t}^{4} L_{x}^{\infty}} \lesssim\left(N_{2} N_{3}\right)^{-\frac{\alpha-1}{2}+\epsilon_{2}}$ is conclusive. When $v_{2}, v_{3}$ are both of type (C), we have the bound (choosing $0<\epsilon<\sigma-\delta_{0}$ )

$$
\begin{aligned}
& \min \left\{\left\|v_{2}\right\|_{L_{t}^{4} L_{x}^{\infty}}\left\|v_{3}\right\|_{L_{t}^{4} L_{x}^{\infty}},\left(N_{2} N_{3}\right)^{\frac{1}{2}-\frac{\alpha}{4}}\left\|v_{2}\right\|_{X^{0, \frac{3}{8}}}\left\|v_{3}\right\|_{X^{0, \frac{3}{8}}}\right\} \\
\lesssim & \min \left\{\left(N_{2} N_{3}\right)^{-(\alpha-1)+\epsilon_{2}}\left(L_{2} L_{3}\right)^{\frac{1}{2}-\nu},\left(N_{2} N_{3}\right)^{\frac{1}{2}-\frac{\alpha}{4}-(\alpha-1)}\left(L_{2} L_{3}\right)^{-\nu}\right\} \\
\lesssim & \left(N_{2} N_{3}\right)^{-(\alpha-1)-2 s \nu+s+\epsilon_{2}-(1+2 \nu) \sigma},
\end{aligned}
$$

which is conclusive since (5.8) holds. Finally we assume that $v_{2}$ is of type $(\mathrm{G})$ and $v_{3}$ is of type (C). Using the bilinear Strichartz inequality we have

$$
\begin{aligned}
& \quad\left|\iint \mathbf{P}_{M_{1}} v \bar{v}_{2} \mathbf{P}_{M_{3}} v_{3} \cdot \mathbf{P}_{M} \bar{w} d x d t\right| \leq\left\|\mathbf{P}_{M_{1}} v \mathbf{P}_{M_{3}} v_{3}\right\|_{L_{t, x}^{2}}\left\|v_{2}\right\|_{L_{t}^{4} L_{x}^{\infty}}\left\|\mathbf{P}_{M} w\right\|_{L_{t}^{4} L_{x}^{2}} \\
& \lesssim\left(M_{1} \wedge M_{3}\right)^{\frac{1}{2}-\frac{\alpha}{4}}\left\|\mathbf{P}_{M_{1}} v\right\|_{X^{0, \frac{3}{8}}}\left\|\mathbf{P}_{M_{3}} v_{3}\right\|_{X^{0, \frac{3}{8}}}\left\|\mathbf{P}_{M} w\right\|_{X^{0, \frac{1}{4}}}\left\|v_{2}\right\|_{L_{t}^{4} L_{x}^{\infty}} .
\end{aligned}
$$

For the non-zero contributions, we must have $M_{3} \lesssim N_{2} \vee N_{3}$, thus when $M_{1} \gg N_{2} \vee N_{3}$, we must have $M_{1} \sim M \gg N_{2} \vee N_{3} \geq M_{3}$, hence

$$
\sum_{\substack{M_{1}, M_{3}, M \\ M \sim M_{1} \gg N_{2} \vee N_{3} \\ M_{3} \leq N_{3}}}\left(M_{1} \wedge M_{3}\right)^{\frac{1}{2}-\frac{\alpha}{4}}\left\|\mathbf{P}_{M_{1}} v\right\|_{X^{0, \frac{3}{8}}}\left\|\mathbf{P}_{M_{3}} v_{3}\right\|_{X^{0, \frac{3}{8}}}\left\|\mathbf{P}_{M} w\right\|_{X^{0, \frac{1}{4}}} \lesssim\|v\|_{X^{0, \frac{3}{8}}} N_{3}^{\frac{1}{2}-\frac{\alpha}{4}}\left\|v_{3}\right\|_{X^{0, \frac{3}{8}}} .
$$

The other contribution can be bounded by

$$
\begin{aligned}
& \sum_{\substack{M_{1}, M_{3}, M \\
M, M_{1} \lesssim N_{2} \vee N_{3}, M_{3} \leq N_{3}}}\left(M_{1} \wedge M_{3}\right)^{\frac{1}{2}-\frac{\alpha}{4}}\left\|\mathbf{P}_{M_{1}} v\right\|_{X^{0, \frac{3}{8}}}\left\|\mathbf{P}_{M_{3}} v_{3}\right\|_{X^{0, \frac{3}{8}}}\left\|\mathbf{P}_{M^{\prime}} w\right\|_{X^{0, \frac{1}{4}}} \\
& \lesssim N_{3}^{\frac{1}{2}-\frac{\alpha}{4}}\left(\log \left(N_{2} \vee N_{3}\right)\right)^{2}\|v\|_{X^{0, \frac{3}{8}}}\left\|v_{3}\right\|_{X^{0, \frac{3}{8}}} .
\end{aligned}
$$


Combining with $(5.9)$, we obtain that

$$
\begin{aligned}
\left|\iint v \bar{v}_{2} v_{3} \cdot \bar{w} d x d t\right| & \lesssim\|v\|_{X^{0, \frac{3}{8}}}\left(\log N_{*}\right)^{4}\left\|v_{2}\right\|_{L_{t}^{4} L_{x}^{\infty}} \min \left\{\left\|v_{3}\right\|_{L_{t}^{4} L_{x}^{\infty}}, N_{3}^{\frac{1}{2}-\frac{\alpha}{4}}\left\|v_{3}\right\|_{X^{0, \frac{3}{8}}}\right\} \\
& \lesssim N_{2}^{-\frac{\alpha-1}{2}+\epsilon_{2}}\left(N_{2} \vee N_{3}\right)^{\epsilon}\|v\|_{X^{0, \frac{3}{8}}} \min \left\{N_{3}^{-(\alpha-1)+\epsilon_{2}} L_{3}^{\frac{1}{2}-\nu}, N_{3}^{\frac{1}{2}-\frac{\alpha}{4}-(\alpha-1)} L_{3}^{-\nu}\right\} \\
& \lesssim N_{2}^{-\frac{\alpha-1}{2}+\epsilon_{2}} N_{3}^{-(\alpha-1)-2 s \nu+s+\epsilon_{2}-(1+2 \nu) \sigma}\left(N_{2} \vee N_{3}\right)^{\epsilon}\|v\|_{X^{0, \frac{3}{8}}},
\end{aligned}
$$

which is conclusive since $(5.8)$ holds. The proof of Lemma 5.1 is now complete.

The proof of (5) and (7) of Proposition 3.7 is an immediate consequence of the above lemma. Now we prove (3) of Proposition 3.7. Consider $\Pi_{N_{0}}^{\perp} \mathcal{I N}_{3}\left(v_{1}, v_{2}, v_{3}\right)$ for $v_{1}, v_{2}, v_{3}$ with characterized parameters $\left(N_{1}, L_{1}\right),\left(N_{2}, L_{2}\right),\left(N_{3}, L_{3}\right)$ with $N_{0} \gg N_{(1)}$. If the projection $\Pi_{N_{0}}^{\perp} \mathcal{I} \mathcal{N}_{3}\left(v_{1}, v_{2}, v_{3}\right)$ does not vanish, then at least one of $v_{1}, v_{2}, v_{3}$ is of type (D), say $v_{1}$. Then we decompose $v_{1}$ as $\sum_{M} \mathbf{P}_{M} v_{1}$, then for $M \geq N_{0}\left(\gg N_{1}\right)$, we have $\left\|\mathbf{P}_{M} v_{1}\right\|_{X^{0, b}} \lesssim M^{-s}$. Applying Lemma 5.1 to $\mathbf{P}_{M} v_{1}$ and using the triangle inequality, we obtain (3) of Proposition 3.7.

\section{LOW MODULATION REDUCTION}

6.1. Modulation reduction for the estimates of operator kernels. For given $v_{2}, v_{3}$ of type $(\mathrm{G}),(\mathrm{C}),(\mathrm{D})$, recall that the kernel $\Theta_{k k^{\prime}}\left(t, t^{\prime}\right)$ of the operator $\mathcal{Q}_{3, N}$ :

$$
w \mapsto \mathcal{Q}_{3, N}(w):=\mathcal{I} \Pi_{N} \mathcal{N}_{3}\left(w, v_{2}, v_{3}\right)
$$

is given by (5.2) with $\Xi_{k k^{\prime}}\left(\mu, \lambda^{\prime}\right)$ given by (5.1). In order to prove Proposition 3.5 and Proposition 3.6 in this section, we will reduce the estimate of the kernel bounds $\|\Theta\|_{S^{b_{1}, b, q}}$ and $\|\Theta\|_{Z^{b_{1}, b, q}}$ to the low-modulation portion which consists of multi-linear expression of discrete sums. Recall that the numerical parameters satisfy $\frac{1}{2}<b_{0}<b<b_{1}, \frac{q}{q-1}-2 b_{1} \ll 1$. We will use the notation $L_{\mu_{0} *}^{r}$ to stand for $L_{\left|\mu_{0}\right| \lesssim N^{\alpha}}^{r}$.

Proposition 6.1. Assume that $\widehat{v_{2}}, \widehat{v_{3}}$ are supported on $\left|k_{j}\right| \lesssim N$. Define $\widetilde{w}_{j}\left(\lambda_{j}, k_{j}\right)=$ $\widetilde{v}_{j}\left(\lambda_{j}, k_{j}\right)\left\langle\lambda_{j}\right\rangle^{\frac{2 b_{0}}{r_{j}^{\prime}}}$, for $j=2,3$ with $r_{j}=2$ or $r_{j}=q$. Then

$$
\begin{aligned}
\left\|\Theta_{k k^{\prime}}\left(\lambda, \lambda^{\prime}\right)\right\|_{Z^{b_{1}, b}} & \lesssim \sup \left\{N^{50\left(2 b_{1}-1\right)} \Upsilon_{N}\left[y^{0} ; w_{2}, w_{3}\right]:\left\|y_{k k^{\prime}}^{0}\left(\lambda, \lambda^{\prime}\right)\right\|_{L_{\lambda, \lambda^{\prime}}^{2} l_{k, k^{\prime}}^{2}} \leq 1\right\}, \\
& +N^{-10}\left\|\widetilde{w}_{2}\right\|_{L_{\lambda}^{r_{2}} l_{k}^{2}}\left\|\widetilde{w}_{3}\right\|_{L_{\lambda}^{r_{3}} l_{k}^{2}}
\end{aligned}
$$

and

$$
\begin{aligned}
\left\|\Theta_{k k^{\prime}}\left(\lambda, \lambda^{\prime}\right)\right\|_{S^{b_{1}, b, q}} & \lesssim \sup \left\{N^{50\left(2 b_{1}-1\right)} \Lambda_{N}\left[y^{0} ; w_{2}, w_{3}\right]:\left\|y_{k k^{\prime}}^{0}\left(\lambda, \lambda^{\prime}\right)\right\|_{l_{k}^{1} L_{\lambda}^{q^{\prime}} L_{\lambda^{\prime}}^{2} l_{k^{\prime}}^{2}} \leq 1\right\} \\
& +N^{-10}\left\|\widetilde{w}_{2}\right\|_{L_{\lambda}^{r_{2}} l_{k}^{2}}\left\|\widetilde{w}_{3}\right\|_{L_{\lambda}^{r_{3}} l_{k}^{2}}
\end{aligned}
$$

where

$$
\Upsilon_{N}\left[y^{0}, w_{2}, w_{3}\right]:=\left\|\sum_{\substack{k, k^{\prime}, k_{2}, k_{3} \\\left(k^{\prime}, k_{2}, k_{3}\right) \in \Gamma(k) \\|k| \leq N}} \widehat{\chi}\left(\mu_{0}-\Phi_{k^{\prime}, k_{2}, k_{3}}\right) y_{k k^{\prime}}^{0}\left(\lambda, \lambda^{\prime}\right) \overline{\widetilde{w}_{2}}\left(\lambda_{2}, k_{2}\right) \widetilde{w}_{3}\left(\lambda_{3}, k_{3}\right)\right\|_{L_{\lambda, \lambda^{\prime}}^{2} L_{\lambda_{2}}^{r_{2}} L_{\lambda_{3}}^{r_{3}} L_{\mu_{0} *}^{20-1}}
$$

and

$$
\Lambda_{N}\left[y^{0} ; w_{2}, w_{3}\right]:=\left\|_{\substack{k, k^{\prime}, k_{2}, k_{3} \\\left(k^{\prime}, k_{2}, k_{3}\right) \in \Gamma(k) \\|k| \leq N}} \widehat{\chi}\left(\mu_{0}-\Phi_{k^{\prime}, k_{2}, k_{3}}\right) y_{k k^{\prime}}^{0}\left(\lambda, \lambda^{\prime}\right) \cdot \widetilde{\widetilde{w}}_{2}\left(\lambda_{2}, k_{2}\right) \widetilde{w}_{3}\left(\lambda_{3}, k_{3}\right)\right\|_{L_{\lambda}^{q^{\prime}} L_{\lambda^{\prime}}^{2} L_{\lambda_{2}}^{r_{2}} L_{\lambda_{3}}^{r_{3}} L_{\mu_{0} *}^{2 b-1}}^{2 b} \|^{2}
$$


Proof. We only prove (6.1), since 6.2 follows from the similar argument (with possible changes of numerical parameters). By duality, it suffices to estimate

$$
\begin{aligned}
\mathfrak{I}:= & \int\langle\lambda\rangle^{b_{1}}\left\langle\lambda^{\prime}\right\rangle^{-b}\left\langle\lambda_{2}\right\rangle^{-\frac{2 b_{0}}{r_{2}^{\prime}}}\left\langle\lambda_{3}\right\rangle^{-\frac{2 b_{0}}{r_{3}^{\prime}}} d \lambda d \lambda d \lambda_{2} d \lambda_{3} \\
& \times \int K(\lambda, \mu) d \mu \sum_{\substack{|k| \leq N,\left|k_{2}\right|,\left|k_{3}\right|{ }_{k} \\
\left(k^{\prime}, k_{2}, k_{3}\right) \in \Gamma(k)}} y_{k k^{\prime}}^{0}\left(\lambda, \lambda^{\prime}\right) \widehat{\chi}\left(\mu-\lambda^{\prime}+\lambda_{2}-\lambda_{3}-\Phi_{k^{\prime}, k_{2}, k_{3}}\right) \overline{\widetilde{w}_{2}}\left(\lambda_{2}, k_{2}\right) \widetilde{w}_{3}\left(\lambda_{3}, k_{3}\right) .
\end{aligned}
$$

From Lemma 2.4 and the triangle inequality, we have

$$
\mathfrak{I} \lesssim A \int \frac{1}{\langle\mu\rangle}\left(\frac{1}{\langle\lambda-\mu\rangle^{A}}+\frac{1}{\langle\lambda\rangle^{A}}\right) \mathcal{T}_{\mu, \lambda, \lambda^{\prime}, \lambda_{2}, \lambda_{3}}\left(w_{2}, w_{3} ; y\right)\langle\lambda\rangle^{b_{1}}\left\langle\lambda^{\prime}\right\rangle^{-b}\left\langle\lambda_{2}\right\rangle^{-\frac{2 b_{0}}{r_{2}^{\prime}}}\left\langle\lambda_{3}\right\rangle^{-\frac{2 b_{0}}{r_{3}^{\prime}}} d \mu d \lambda d \lambda^{\prime} d \lambda_{2} d \lambda_{3},
$$

where

$$
\mathcal{T}_{\mu, \lambda^{\prime}, \lambda, \lambda_{2}, \lambda_{3}}\left(w_{2}, w_{3} ; y\right):=\left|\sum_{\substack{|k| \leq N,\left|k_{2}\right|,\left|k_{3}\right| \leq N \\\left(k^{\prime}, k_{2}, k_{3}\right) \in \Gamma(k)}} y_{k k^{\prime}}^{0}\left(\lambda, \lambda^{\prime}\right) \widehat{\chi}\left(\mu-\lambda^{\prime}+\lambda_{2}-\lambda_{3}-\Phi_{k^{\prime}, k_{2}, k_{3}}\right) \overline{\widetilde{w}_{2}}\left(\lambda_{2}, k_{2}\right) \widetilde{w}_{3}\left(\lambda_{3}, k_{3}\right)\right| .
$$

Here and in the sequel, $\mathcal{T}$ stands for $\mathcal{T}_{\mu, \lambda, \lambda^{\prime}, \lambda_{2}, \lambda_{3}}\left(v_{2}, v_{3} ; y\right)$ when there is no risk of confusion.

\section{- Contribution from the integration of $\mathcal{T}$ against $\frac{1}{\langle\mu\rangle\langle\lambda\rangle^{A}}$ :}

We split (for fixed $\left.\lambda, \lambda^{\prime}, \lambda_{2}, \lambda_{3}\right)$ the integration as $\int_{\left|\mu-\left(\lambda^{\prime}-\lambda_{2}+\lambda_{3}\right)\right| \gg N^{\alpha}}$ and $\int_{\left|\mu-\left(\lambda^{\prime}-\lambda_{2}+\lambda_{3}\right)\right| \lesssim N^{\alpha}}$. Note that for non-zero contributions in the summation of $k, k^{\prime}, k_{2}, k_{3},\left|\Phi_{k^{\prime}, k_{2}, k_{3}}\right| \lesssim N^{\alpha}$, we have from the rapid decay of $\widehat{\chi}(\cdot)$ that

$$
\left|\widehat{\chi}\left(\mu-\lambda^{\prime}+\lambda_{2}-\lambda_{3}-\Phi_{k^{\prime}, k_{2}, k_{3}}\right)\right| \lesssim_{A}\left\langle\mu-\left(\lambda^{\prime}-\lambda_{2}+\lambda_{3}\right)\right\rangle^{-A}
$$

if $\left|\mu-\left(\lambda^{\prime}-\lambda_{2}+\lambda_{3}\right)\right| \gg N^{\alpha}$, hence

$$
\begin{aligned}
& \int_{\left|\mu-\left(\lambda^{\prime}-\lambda_{2}+\lambda_{3}\right)\right| \gg N^{\alpha}} \frac{1}{\langle\mu\rangle\langle\lambda\rangle^{A}} \cdot \mathcal{T} d \mu \\
& \lesssim \int_{\left|\mu-\left(\lambda^{\prime}-\lambda_{2}+\lambda_{3}\right)\right| \gg N^{\alpha}} \frac{N \cdot N^{\frac{1}{2}} N^{\frac{1}{2}} d \mu}{\langle\lambda\rangle^{A}\langle\mu\rangle\left\langle\mu-\left(\lambda^{\prime}-\lambda_{2}+\lambda_{3}\right)\right\rangle^{-A}}\left\|\widetilde{w}_{2}\left(\lambda_{2}, \cdot\right)\right\|_{l_{k_{2}}^{2}}\left\|\widetilde{w}_{3}\left(\lambda_{3}, \cdot\right)\right\|_{l_{k_{3}}^{2}}\left\|y_{k k^{\prime}}^{0}\left(\lambda, \lambda^{\prime}\right)\right\|_{l_{k, k^{\prime}}^{2}} \\
& \lesssim\langle\lambda\rangle^{-A} N^{2-A \alpha}\left\|\widetilde{w}_{2}\left(\lambda_{2}, \cdot\right)\right\|_{l_{k_{2}}^{2}}\left\|\widetilde{w}_{3}\left(\lambda_{3}, \cdot\right)\right\|_{l_{k_{3}}^{2}}\left\|y_{k k^{\prime}}^{0}\left(\lambda, \lambda^{\prime}\right)\right\|_{l_{k, k^{\prime}}^{2}} \cdot
\end{aligned}
$$

Choosing $A=200$, say, and multiplying by $\langle\lambda\rangle^{b_{1}}\left\langle\lambda^{\prime}\right\rangle^{-b}\left\langle\lambda_{2}\right\rangle^{-\frac{2 b_{0}}{r_{2}^{\prime}}}\left\langle\lambda_{3}\right\rangle^{-\frac{2 b_{0}}{r_{3}^{\prime}}}$ and integrating in $\lambda, \lambda^{\prime}, \lambda_{2}, \lambda_{3}$, this contribution for $\mathfrak{I}$ is bounded by

$$
N^{-100}\left\|\widetilde{w}_{2}\right\|_{L_{\lambda}^{r_{2}} l_{k}^{2}}\left\|\widetilde{w}_{3}\right\|_{L_{\lambda}^{r_{3}} l_{k}^{2}}\left\|y_{k k^{\prime}}^{0}\left(\lambda, \lambda^{\prime}\right)\right\|_{L_{\lambda, \lambda^{\prime}}^{2} l_{k, k^{\prime}}^{2}} .
$$

The other term can be estimated as

$$
\int_{\left|\mu-\left(\lambda^{\prime}-\lambda_{2}+\lambda_{3}\right)\right| \lesssim N^{\alpha}} \frac{1}{\langle\mu\rangle\langle\lambda\rangle^{A}} \cdot \mathcal{T} d \mu \lesssim b, b_{1} \frac{1}{\langle\lambda\rangle^{A}}\left\|\mathcal{T}_{\mu, \lambda^{\prime}, \lambda, \lambda_{2}, \lambda_{3}}\right\|_{L_{\mu:\left|\mu-\left(\lambda^{\prime}-\lambda_{2}+\lambda_{3}\right)\right| \lesssim N^{\alpha}}^{\frac{4}{2 b-1}}} .
$$

Again, choosing $A=200$ and multiplying by $\langle\lambda\rangle^{b_{1}}\left\langle\lambda^{\prime}\right\rangle^{-b}\left\langle\lambda_{2}\right\rangle^{-\frac{2 b_{0}}{r_{2}^{\prime}}}\left\langle\lambda_{3}\right\rangle^{-\frac{2 b_{0}}{r_{3}^{\prime}}}$ and integrating in $\lambda, \lambda^{\prime}, \lambda_{2}, \lambda_{3}$, this contribution for $\mathfrak{I}$ is bounded by the first term of the right side of (6.1).

- Contribution from the integration of $\mathcal{T}$ against $\frac{1}{\langle\mu\rangle\langle\lambda-\mu\rangle^{A}}$ :

As the previous case, we split (for fixed $\lambda, \lambda^{\prime}, \lambda_{2}, \lambda_{3}$ ) the integration in $\int_{|\mu| \gg\left|\lambda^{\prime}-\lambda_{2}+\lambda_{3}\right|+N^{\alpha}}$ 
and $\int_{|\mu| \lesssim\left|\lambda^{\prime}-\lambda_{2}+\lambda_{3}\right|+N^{\alpha}}$. Using Cauchy-Schwartz, we have

$$
\int \frac{\left\|y_{k, k^{\prime}}^{0}\left(\lambda, \lambda^{\prime}\right)\right\|_{l_{k, k^{\prime}}^{2}}\langle\lambda\rangle^{b_{1}}}{\langle\lambda-\mu\rangle^{A}} d \lambda \lesssim\left\|y_{k, k^{\prime}}^{0}\left(\lambda, \lambda^{\prime}\right)\right\|_{L_{\lambda}^{2} l_{k, k^{\prime}}^{2}}\langle\mu\rangle^{b_{1}}
$$

then by similar manipulations as in (6.6), the contribution from the region $\mid \mu-\left(\lambda^{\prime}-\right.$ $\left.\lambda_{2}+\lambda\right) \mid \gg N^{\alpha}$ yields the second term of the right side of $(6.1)$ as an error. The main contribution comes from the region $\left|\mu-\left(\lambda^{\prime}-\lambda_{2}+\lambda_{3}\right)\right| \lesssim N^{\alpha}$. We further split the integration of $\mathfrak{I}$ :

$$
\int\left\langle\lambda^{\prime}\right\rangle^{-b}\left\langle\lambda_{2}\right\rangle^{-\frac{2 b}{r_{2}^{\prime}}}\left\langle\lambda_{3}\right\rangle^{-\frac{2 b}{r_{3}^{\prime}}} d \lambda^{\prime} d \lambda_{2} d \lambda_{3}\left(\int_{\substack{|\lambda-\mu| \ll N^{\alpha} \\ \mid \mu-\left(\lambda^{\prime}-\lambda_{2}+\lambda_{3}\right) \lesssim N^{\alpha}}}+\int_{\substack{\left|\lambda-\left(\lambda^{\prime}-\lambda_{2}+\lambda_{3}\right) \lesssim N^{\alpha}\\\right|}} \frac{\langle\lambda\rangle^{b_{1}}}{\langle\mu\rangle\langle\lambda-\mu\rangle^{A}} \cdot \mathcal{T} d \mu d \lambda .\right.
$$

Taking $A=200$ and applying Hölder for the integration in $\mu$ and using Lemma 2.5, we have

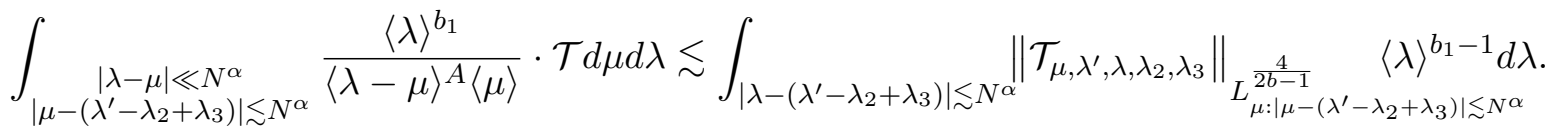

Alternatively, applying Cauchy-Schwartz for the integration in $\lambda$ and using Lemma 2.5. we have

$$
\int_{\substack{|\lambda-\mu| \ll N^{\alpha} \\\left|\mu-\left(\lambda^{\prime}-\lambda_{2}+\lambda_{3}\right)\right| \lesssim N^{\alpha}}} \frac{\langle\lambda\rangle^{b_{1}}}{\langle\lambda-\mu\rangle^{A}\langle\mu\rangle} \cdot \mathcal{T} d \mu d \lambda \lesssim \int_{\left|\mu-\left(\lambda^{\prime}-\lambda_{2}+\lambda_{3}\right)\right| \lesssim N^{\alpha}} \frac{\left\|\mathcal{T}_{\mu, \lambda^{\prime}, \lambda, \lambda_{2}, \lambda_{3}}\right\|_{L_{\lambda}^{2}}}{\langle\mu\rangle^{1-b_{1}}} d \mu .
$$

Multiplying by $\left\langle\lambda^{\prime}\right\rangle^{-b}\left\langle\lambda_{2}\right\rangle^{-\frac{2 b_{0}}{r_{2}^{\prime}}}\left\langle\lambda_{3}\right\rangle^{-\frac{2 b_{0}}{r_{3}^{\prime}}} \mathbf{1}_{\left|\lambda^{\prime}-\lambda_{2}+\lambda_{3}\right| \leq N^{100}}$ to the left side of (6.7) and integrating in $\lambda^{\prime}, \lambda_{2}, \lambda_{3}$, we have

$$
\begin{aligned}
& \int\left\langle\lambda^{\prime}\right\rangle^{-b}\left\langle\lambda_{2}\right\rangle^{-\frac{2 b_{0}}{r_{2}^{\prime}}}\left\langle\lambda_{3}\right\rangle^{-\frac{2 b_{0}}{r_{3}^{\prime}}} \mathbf{1}_{\left|\lambda^{\prime}-\lambda_{2}+\lambda_{3}\right| \leq N^{100}} \cdot(\text { l.h.s. of }[6.7)) d \lambda^{\prime} d \lambda_{2} d \lambda_{3} \\
\lesssim & \int_{|\lambda| \lesssim N^{100}}\left\|\mathcal{T}_{\mu, \lambda^{\prime}, \lambda, \lambda_{2}, \lambda_{3}}\right\|_{L_{\mu:\left|\mu-\left(\lambda^{\prime}-\lambda_{2}+\lambda_{3}\right)\right| \lesssim N^{\alpha}}^{2 b-1}} \frac{1}{\langle\lambda\rangle^{1-b_{1}}}\left\langle\lambda^{\prime}\right\rangle^{-b}\left\langle\lambda_{2}\right\rangle^{-\frac{2 b_{0}}{r_{2}^{\prime}}}\left\langle\lambda_{3}\right\rangle^{-\frac{2 b_{0}}{r_{3}^{\prime}}} d \lambda d \lambda^{\prime} d \lambda_{2} d \lambda_{3} \\
\lesssim & N^{100\left(b_{1}-\frac{1}{2}\right)}\left\|\mathcal{T}_{\mu, \lambda^{\prime}, \lambda, \lambda_{2}, \lambda_{3}}\right\|_{L_{\lambda^{\prime}, \lambda}^{2} L_{\lambda_{2}}^{r_{2}} L_{\lambda_{3}}^{r_{3}} L_{\mu:\left|\mu-\left(\lambda^{\prime}-\lambda_{2}+\lambda_{3}\right)\right| \lesssim N^{\alpha}}^{\frac{4}{2 b-1}}} \cdot
\end{aligned}
$$

Multiplying by $\left\langle\lambda^{\prime}\right\rangle^{-b}\left\langle\lambda_{2}\right\rangle^{-\frac{2 b_{0}}{r_{2}^{\prime}}}\left\langle\lambda_{3}\right\rangle^{-\frac{2 b_{0}}{r_{3}^{\prime}}} \mathbf{1}_{\left|\lambda^{\prime}-\lambda_{2}+\lambda_{3}\right|>N^{100}}$ to the left side of (6.8), we obtain that

$$
\begin{aligned}
& \left.\int\left\langle\lambda^{\prime}\right\rangle^{-b}\left\langle\lambda_{2}\right\rangle^{-\frac{2 b_{0}}{r_{2}^{\prime}}}\left\langle\lambda_{3}\right\rangle^{-\frac{2 b_{0}}{r_{3}^{\prime}}} \mathbf{1}_{\left|\lambda^{\prime}-\lambda_{2}+\lambda_{3}\right|>N^{100}} \cdot(\text { r.h.s. of } 6.8)\right) d \lambda^{\prime} d \lambda_{2} d \lambda_{3} \\
\lesssim & \int_{|\mu| \sim N^{100}} \frac{\left\|\mathcal{T}_{\mu, \lambda^{\prime}, \lambda, \lambda_{2}, \lambda_{3}}\right\|_{L_{\lambda}^{2}}}{\langle\mu\rangle^{1-b_{1}}}\left\langle\lambda^{\prime}\right\rangle^{-b}\left\langle\lambda_{2}\right\rangle^{-\frac{2 b_{0}}{r_{2}^{\prime}}}\left\langle\lambda_{3}\right\rangle^{-\frac{2 b_{0}}{r_{3}^{\prime}}} d \mu d \lambda^{\prime} d \lambda_{2} d \lambda_{3} \\
\leq & N^{-100\left(1-b_{1}\right)}\left\|\mathcal{T}_{\mu, \lambda^{\prime}, \lambda, \lambda_{2}, \lambda_{3}}\right\|_{L_{\lambda^{\prime}}^{2} L_{\lambda_{2}}^{r_{2}} L_{\lambda_{3}}^{r_{3}} L_{\mu}^{1} L_{\lambda}^{2}} .
\end{aligned}
$$

By definition and Cauchy-Schwartz,

$$
\begin{aligned}
& N^{-100\left(1-b_{1}\right)}\left\|\mathcal{T}_{\mu, \lambda^{\prime}, \lambda, \lambda_{2}, \lambda_{3}}\right\|_{L_{\lambda^{\prime}}^{2} L_{\lambda_{2}}^{r_{2}} L_{\lambda_{3}}^{r_{3}} L_{\mu}^{1} L_{\lambda}^{2}} \\
\leq & N^{-100\left(1-b_{1}\right)}\left\|_{|k|,\left|k^{\prime}\right|,\left|k_{2}\right|,\left|k_{3}\right| \lesssim N}\right\| \widehat{\chi}\left\|_{L_{\mu}^{1}}\right\| y_{k, k^{\prime}}^{0}\left(\lambda, \lambda^{\prime}\right)\left\|_{L_{\lambda}^{2}} \overline{\widehat{w}_{2}}\left(\lambda_{2}, k_{2}\right) \widehat{w}_{3}\left(\lambda_{3}, k_{3}\right)\right\|_{L_{\lambda^{\prime}}^{2} L_{\lambda_{2}}^{r_{2}} L_{\lambda_{3}}^{r_{3}}} \\
\lesssim & N^{2-100\left(1-b_{1}\right)}\left\|y_{k, k^{\prime}}^{0}\left(\lambda, \lambda^{\prime}\right)\right\|_{l_{k, k^{\prime}}^{2} L_{\lambda, \lambda^{\prime}}^{2}}\left\|\widetilde{w}_{2}\left(\lambda_{2}, k_{2}\right)\right\|_{L_{\lambda_{2}}^{r_{2}} l_{k_{2}}^{2}}\left\|\widetilde{w}_{3}\left(\lambda_{3}, k_{3}\right)\right\|_{L_{\lambda_{3}}^{r_{3}} l_{k_{3}}^{2}},
\end{aligned}
$$

hence it can be bounded by the second error term of the right side of (6.1). 
For the integration over $|\lambda-\mu| \gtrsim N^{\alpha}$, we further split it in three parts: $|\lambda|>2|\mu|,|\lambda|<$ $\frac{1}{2}|\mu|$ and $\frac{1}{2}|\mu| \leq|\lambda| \leq 2|\mu|$. For $|\lambda|<\frac{1}{2}|\mu|$, we have $|\mu| \sim|\lambda-\mu| \gtrsim N^{\alpha}$, thus

$$
\int_{\substack{|\lambda-\mu| \gtrsim N^{\alpha},|\lambda|<\frac{1}{2}|\mu|,\left|\mu-\left(\lambda^{\prime}-\lambda_{2}+\lambda_{3}\right)\right| \lesssim N^{\alpha}}} \frac{\langle\lambda\rangle^{b_{1}}}{\langle\lambda-\mu\rangle^{A}\langle\mu\rangle} \cdot \mathcal{T} d \lambda d \mu \lesssim \int_{|\mu| \gtrsim N^{\alpha}} \frac{\left\|\mathcal{T}_{\mu, \lambda^{\prime}, \lambda, \lambda_{2}, \lambda_{3}}\right\|_{L_{\lambda}^{2}}}{\langle\mu\rangle^{A-b_{1}}} d \mu .
$$

Multiplying by $\left\langle\lambda^{\prime}\right\rangle^{-b}\left\langle\lambda_{2}\right\rangle^{-\frac{2 b_{0}}{r_{2}^{\prime}}}\left\langle\lambda_{3}\right\rangle^{-\frac{2 b_{0}}{r_{3}^{\prime}}}$ and integrating in $\lambda^{\prime}, \lambda_{2}, \lambda_{3}$ then using CauchySchwartz and Minkowski, the above term is bounded by the second error term of the right side of (6.1). Similarly, for the case $|\lambda|>2|\mu|$, we have $|\lambda| \sim|\lambda-\mu| \gtrsim N^{\alpha}$. This gives us an error term as the second term of the right side of $(6.1)$, provided that $A$ is chosen large enough. Finally, for the case $\frac{1}{2}|\mu| \leq|\lambda| \leq 2|\mu|$, using Hölder and Lemma 2.5 (and we write $\langle\lambda-\mu\rangle^{A} \gtrsim\langle\lambda-\mu\rangle^{A / 2} N^{A \alpha / 2}$ for $A \gg 1$ ), we have

$$
\begin{aligned}
& \int_{\frac{1}{2}|\lambda-\mu| \geq|\lambda| \leq 2|\mu|} \frac{\langle\lambda\rangle^{b_{1}}}{\langle\lambda-\mu\rangle^{A}\langle\mu\rangle} \mathcal{T} \mathbf{1}_{\left|\mu-\left(\lambda^{\prime}-\lambda_{2}+\lambda_{3}\right)\right| \lesssim N^{\alpha}} d \lambda d \mu \\
& \lesssim N^{-A \alpha / 2} \int_{\mid \mu-\left(\lambda^{\prime}-\lambda_{2}+\lambda_{3}\right) \lesssim N^{\alpha}}\left\|\mathbf{1}_{|\mu| \sim|\lambda|} \mathcal{T}_{\mu, \lambda^{\prime}, \lambda, \lambda_{2}, \lambda_{3}}\right\|_{L_{\lambda}^{2}} \cdot \frac{d \mu}{\langle\mu\rangle^{1-b_{1}}} \\
& \lesssim N^{-A \alpha / 2}\left\|\langle\mu\rangle^{1-b_{1}}\right\|_{L_{\mu}^{3}}\left\|\mathbf{1}_{|\mu| \sim|\lambda|} \mathcal{T}_{\mu, \lambda^{\prime}, \lambda, \lambda_{2}, \lambda_{3}}\right\|_{L_{\mu}^{3 / 2} L_{\lambda}^{2}} .
\end{aligned}
$$

Multiplying by $\left\langle\lambda^{\prime}\right\rangle^{-b}\left\langle\lambda_{2}\right\rangle^{-\frac{2 b_{0}}{r_{2}^{\prime}}}\left\langle\lambda_{3}\right\rangle^{-\frac{2 b_{0}}{r_{3}^{\prime}}}$, integrating in $\lambda^{\prime}, \lambda_{2}, \lambda_{3}$ and using Cauchy-Schwartz, this contribution can be controlled by

$$
\begin{aligned}
& N^{-\frac{A \alpha}{2}}\left\|_{|k|,\left|k^{\prime}\right|,\left|k_{2}\right|,\left|k_{3}\right| \lesssim N} \widehat{\chi}\left(\mu-\lambda^{\prime}+\lambda_{2}-\lambda_{3}-\Phi_{k^{\prime}, k_{2}, k_{3}}\right) y_{k, k^{\prime}}^{0}\left(\lambda, \lambda^{\prime}\right) \widetilde{\widetilde{w}_{2}}\left(\lambda_{2}, k_{2}\right) \widetilde{w}_{3}\left(\lambda_{3}, k_{2}\right)\right\|_{L_{\lambda^{\prime}}^{2}{ }_{\lambda_{2}}^{r_{2}} L_{\lambda_{3}}^{r_{3}} L_{\mu}^{\frac{3}{2}} L_{\lambda}^{2}} \\
& \leq N^{-\frac{A \alpha}{2}}\left\|\sum_{|k|,\left|k^{\prime}\right|,\left|k_{2}\right|,\left|k_{3}\right| \lesssim N}\right\| \widehat{\chi}\left\|_{L_{\mu}^{\frac{3}{2}}}\right\| y_{k, k^{\prime}}^{0}\left(\lambda, \lambda^{\prime}\right)\left\|_{L_{\lambda}^{2}} \overline{\widetilde{w}_{2}}\left(\lambda_{2}, k_{2}\right) \widetilde{w}_{3}\left(\lambda_{3}, k_{2}\right)\right\|_{L_{\lambda^{\prime}}^{2} L_{\lambda_{2}}^{r_{2}} L_{\lambda_{3}}^{r_{3}}} \\
& \lesssim N^{-A \alpha / 2+2}\left\|y_{k, k^{\prime}}^{0}\left(\lambda, \lambda^{\prime}\right)\right\|_{l_{k, k^{\prime}}^{2} L_{\lambda, \lambda^{\prime}}^{2}}\left\|\widetilde{w}_{2}\left(\lambda_{2}, k_{2}\right)\right\|_{L_{\lambda_{2}}^{r_{2}} l_{k_{2}}^{2}}\left\|\widetilde{w}_{3}\left(\lambda_{3}, k_{3}\right)\right\|_{L_{\lambda_{3}}^{r_{3}} l_{k_{3}}^{2}},
\end{aligned}
$$

and it can be controlled by the second error term of the right side of (6.1)). This completes the proof of Proposition 6.1.

6.2. Modulation reduction for the trilinear estimates. Assume that $v_{j} \in X^{0, b_{0}} \cap$ $X_{\infty, q}^{0, \frac{2 b_{0}}{q^{\prime}}} \operatorname{such}$ that $\operatorname{supp}\left(\widetilde{v}_{j}\right) \subset\left\{\left|k_{j}\right| \lesssim N_{j}\right\}$, for $j=1,2,3$. Let $\widetilde{w}_{j}^{\left(r_{j}\right)}=\langle\lambda\rangle^{\frac{2 b_{0}}{r_{j}^{\prime}}} \widetilde{v}_{j}$, and without loss of generality, we assume that $v_{j}=\chi_{1}(t) v_{j}$, for $j=1,2,3$, where $\chi_{1} \in C_{c}^{\infty}(\mathbb{R})$. Let $\chi(t)$ be another time cut-off function such that $\chi \chi_{1}=\chi_{1}$.

Proposition 6.2. Adapting to the notations above, we have for any $\epsilon>0$,

$$
\begin{aligned}
& \left\|\mathcal{N}_{3}\left(v_{1}, v_{2}, v_{3}\right)\right\|_{X^{0, b_{1}-1}} \lesssim \epsilon N_{(1)}^{-100} \prod_{j=1}^{3}\left\|v_{j}\right\|_{X^{0, b}} \\
+ & \left\|\sum_{\substack{\left(k_{1}, k_{2}, k_{3}\right) \in \Gamma(k) \\
\left|k_{j}\right| \lesssim N_{j}, j=1,2,3}} \widehat{\chi}\left(\mu_{0}-\Phi_{k_{1}, k_{2}, k_{3}}\right) \widetilde{w}_{1}^{\left(r_{1}\right)}\left(\lambda_{1}, k_{1}\right) \overline{\widetilde{w}_{2}^{\left(r_{2}\right)}}\left(\lambda_{2}, k_{2}\right) \widetilde{w}_{3}^{\left(r_{3}\right)}\left(\lambda_{3}, k_{3}\right)\right\|_{L_{\lambda_{1}}^{r_{1}} L_{\lambda_{2}}^{r_{2}} L_{\lambda_{3}}^{r_{3}} L_{\left|\mu_{0}\right| \lesssim N_{(1)}^{\alpha}}^{2 b_{1}+2 \epsilon-1}} l_{k}^{2}
\end{aligned}
$$

Proof. Since there is no significant importance of the conjugate bar on $\widetilde{v}_{2}$, we will omit it in the proof. By duality, for $v=\chi(t) v \in X^{0,1-b_{1}},\left\|\langle\lambda\rangle^{1-b_{1}} \widetilde{v}(\lambda, k)\right\|_{L_{\lambda}^{2} l_{k}^{2}} \lesssim 1$, we need to 
estimate

$$
\begin{aligned}
& \sum_{\substack{|k| \leqslant N_{(1)},\left|k_{j}\right| \leq N_{j}, j=1,2,3 \\
\left(k_{1}, k_{2}, k_{3}\right) \in \Gamma(k)}} \int \prod_{j=1}^{3} \widehat{v}_{j}\left(\tau_{j}, k_{j}\right) d \tau_{j} \int \widehat{v}\left(\tau_{4}, k\right) \widehat{\chi}\left(\tau_{1}-\tau_{2}+\tau_{3}-\tau_{4}\right) d \tau_{4} \\
= & \sum_{\substack{|k| \lesssim N_{(1)},\left|k_{j}\right| \leq N_{j}, j=1,2,3 \\
\left(k_{1}, k_{2}, k_{3}\right) \in \Gamma(k)}} \int\left(\prod_{j=1}^{3}\left\langle\lambda_{j}\right\rangle^{-\frac{2 b_{0}}{r_{j}^{\prime}}} \widetilde{w}_{j}^{\left(r_{j}\right)}\left(\lambda_{j}, k_{j}\right) d \lambda_{j}\right) \cdot \widetilde{v}(\lambda, k) \widehat{\chi}\left(\lambda_{1}-\lambda_{2}+\lambda_{3}-\lambda-\Phi_{k_{1}, k_{2}, k_{3}}\right) d \lambda .
\end{aligned}
$$

By Cauchy-Schwartz, 6.10 is bounded by

$$
\int\left(\prod_{j=1}^{3}\left\langle\lambda_{j}\right\rangle^{-\frac{2 b_{0}}{r_{j}^{\prime}}} d \lambda_{j}\right)\left\|\langle\lambda\rangle^{1-b_{1}} \widetilde{v}\right\|_{L_{\lambda}^{2} l_{k}^{2}} \cdot\left\|\langle\lambda\rangle^{-\left(1-b_{1}\right)} \mathcal{M}_{\lambda, \lambda_{1}, \lambda_{2}, \lambda_{3}}\left(v_{1}, v_{2}, v_{3}\right)\right\|_{L_{\lambda}^{2} l_{k}^{2}}
$$

where

$$
\mathcal{M}_{\lambda, \lambda_{1}, \lambda_{2}, \lambda_{3}}\left(v_{1}, v_{2}, v_{3}\right):=\sum_{\substack{\left|k_{j}\right| \leq N_{j}, j=1,2,3 \\\left(k_{1}, k_{2}, k_{3}\right) \in \Gamma(k)}} \widetilde{w}_{1}^{\left(r_{1}\right)}\left(\lambda_{1}, k_{1}\right) \widetilde{w}_{2}^{\left(r_{2}\right)}\left(\lambda_{2}, k_{2}\right) \widetilde{w}_{3}^{r_{3}}\left(\lambda_{3}, k_{3}\right) \widehat{\chi}\left(\lambda-\lambda_{1}+\lambda_{2}-\lambda_{3}-\Phi_{k_{1}, k_{2}, k_{3}}\right) .
$$

To simplify the notation, we denote $\mathcal{M}_{\lambda, \lambda_{1}, \lambda_{2}, \lambda_{3}}\left(v_{1}, v_{2}, v_{3}\right)$ simply by $\mathcal{M}$, when there is no risk of confusing. By Hölder, we have

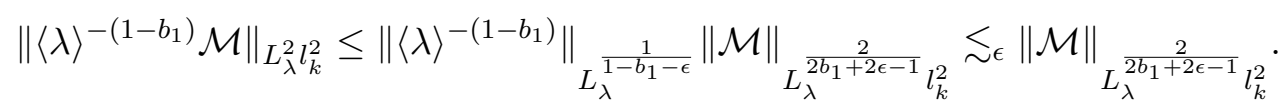

For fixed $\lambda_{1}, \lambda_{2}, \lambda_{3}$, we split the region of integration (in $\lambda$ ) in $\left|\lambda-\lambda_{1}+\lambda_{2}-\lambda_{3}\right| \gg N_{(1)}^{\alpha}$ and $\left|\lambda-\lambda_{1}+\lambda_{2}-\lambda_{3}\right| \lesssim N_{(1)}^{\alpha}$. For $\left|\lambda-\lambda_{1}+\lambda_{2}-\lambda_{3}\right| \gg N_{(1)}^{\alpha}$, using the rapid decay of $\widehat{\chi}(\cdot)$, we have

$$
\left|\widehat{\chi}\left(\lambda-\lambda_{1}+\lambda_{2}-\lambda_{3}+\Phi_{k_{1}, k_{2}, k_{3}}\right)\right| \lesssim_{A}\left\langle\lambda-\lambda_{1}+\lambda_{2}-\lambda_{3}\right\rangle^{-A_{1}} \mathbf{1}_{\left|\lambda-\lambda_{1}+\lambda_{2}-\lambda_{3}\right| \gg N_{(1)}^{\alpha}} .
$$

By taking $A \gg 1$, we have

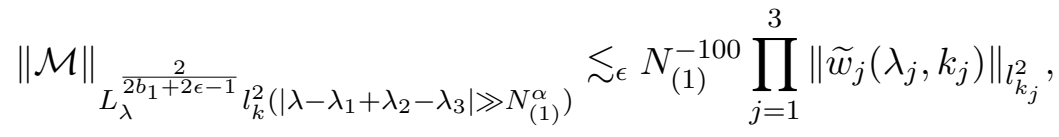

provided that $A \gg 1$. Hence this contribution in 6.10 is an error and can be bounded by the first term on the right side of $(6.9)$. The other term can be bounded by

$$
\|\mathcal{M}\|_{L_{\lambda}^{\frac{2}{2 b_{1}+2 \epsilon-1}} l_{k}^{2}\left(\left|\lambda-\lambda_{1}+\lambda_{2}-\lambda_{3}\right| \lesssim N_{(1)}^{\alpha}\right)}
$$

as desired. This completes the proof of Proposition 6.2 .

\section{Multilinear estimate FOR the Kernel $\mathcal{P}_{N, L}^{+}$}

The goal of this section is to prove Proposition 3.5 and Proposition 3.6. Recall that $\Theta_{k, k_{1}}^{N_{1}, L}\left(t, t_{1}\right)$ is the kernel of the operator

$$
\mathcal{P}_{N_{1}, L}^{+}:=\chi_{T}(t) \Pi_{N_{1}} \mathcal{I}\left[\mathcal{N}_{3}\left(\Pi_{N_{1}} \cdot \Pi_{L} v_{L}^{\#}, \Pi_{L} v_{L}^{\#}\right)-\Pi_{N_{1}} \mathcal{N}_{3}\left(\Pi_{N_{1}} \cdot \Pi_{\frac{L}{2}} v_{\frac{L}{2}}^{\#}, \Pi_{\frac{L}{2}} v_{\frac{L}{2}}^{\#}\right)\right]
$$

where $L<N_{1}^{1-\delta}$. Since we will only estimate the kernel restricted to $|k|,\left|k_{1}\right| \geq \frac{N_{1}}{4}$, by abusing the notation, we will sometimes regard $\Theta_{k, k_{1}}^{N_{1}, L}\left(t, t_{1}\right)$ as $\Theta_{k, k_{1}}\left(t, t_{1}\right)^{N_{1}, L} \mathbf{1}_{|k|,\left|k_{1}\right| \sim N_{1}}$. By decomposing $v_{L}^{\#}$ as sums of terms $v_{j}$ of type $(\mathrm{D}),(\mathrm{G})$ or $(\mathrm{C})$ with corresponding characterized parameters $\left(N_{2}, L_{2}\right)$, we can write $\Theta_{k k_{1}}^{N_{1}, L}\left(t, t_{1}\right) \mathbf{1}_{|k|,\left|k_{1}\right| \sim N_{1}}$ as the sum of kernels of the linear combination of operators

$$
\Pi_{N_{1}} \mathcal{I N}_{3}\left(\cdot, \Pi_{L} v_{2}, \Pi_{L} v_{3}\right),
$$


where $v_{2}, v_{3}$ are of type $(\mathrm{G}),(\mathrm{C})$ or $(\mathrm{D})$ with characterized parameters $\left(N_{2}, L_{2}\right),\left(N_{3}, L_{3}\right)$, with respectively, satisfying $N_{2} \vee N_{3} \sim L$. To prove Proposition 3.5 and Proposition 3.6. we will first provide estimates for each single piece and then sum them up.

7.1. Notational simplifications. We fix another numerical parameter (remember that $q$ is reserved in Remark 3.1):

$$
q_{0}:=\frac{2}{2 b_{1}-1} \text { or } \frac{q}{2 b_{1}-1}
$$

in this section, which will be clear in different contexts. The importance is that $q_{0} \gg 1$. As in the previous section, we will write $L_{\mu_{0} *}^{r}$ to stand for $L_{\left|\mu_{0}\right| \lesssim N_{1}^{\alpha}}^{r}$. Let $\mathcal{C}_{j}$ be the $\sigma$-algebra $\mathcal{B}_{\leq L_{j}}$ which is independent of the $\sigma$-algebra generated by $\left\{g_{k_{j}^{*}}:\left|k_{j}^{*}\right| \sim N_{j}\right\}$, we denote by $\mathbb{E}^{\mathcal{C}_{j}}[\cdot]=\mathbb{E}\left[\cdot \mid \mathcal{C}_{j}\right]$.

Before doing the estimates, we observe that modulo terms of

$$
O_{\epsilon}\left(N_{1}^{-\frac{1}{\epsilon}+10}\right)\left\|y_{k, k_{1}}^{0}\left(\lambda, \lambda_{1}\right)\right\|_{l_{k, k_{1}}^{2}}\left\|\widetilde{w}_{2}\left(\lambda_{2}, k_{2}\right)\right\|_{l_{k_{2}}^{2}}\left\|\widetilde{w}_{3}\left(\lambda_{3}, k_{3}\right)\right\|_{l_{k_{3}}^{2}},
$$

we may replace $\widehat{\chi}_{0}\left(\mu_{0}-\Phi_{k_{1}, k_{2}, k_{3}}\right)$ by $\mathbf{1}_{\Phi_{k_{1}, k_{2}, k_{3}}=\mu_{0}+O\left(N_{1}^{\epsilon}\right)}$ for any $\epsilon>0$ and we denote by

$$
S_{k_{1}, k_{2}, k_{3}}:=\mathbf{1}_{\Phi_{k_{1}, k_{2}, k_{3}}=\mu_{0}+O\left(N_{1}^{\epsilon}\right)} \mathbf{1}_{k_{2} \neq k_{1}, k_{3}} \text {. }
$$

Note that $S_{k_{1}, k_{2}, k_{3}}$ depends on $\mu_{0}$, but we will always have uniform estimates for $S_{k_{1}, k_{2}, k_{3}}$ in $\mu_{0}$. Therefore, we will not mention this dependence explicitly. Here $\epsilon>0$ is another free (small) parameter which will be fixed later, according to different contexts. Furthermore, when $v_{j}$ is of type $(C)$, we may replace it by

$$
\widetilde{v}_{j}\left(\lambda_{j}, k_{j}\right)=\sum_{\left|k_{j}^{*}\right| \sim N_{j}} \mathbf{1}_{\left|k_{j}-k_{j}^{*}\right| \leq L_{j} N_{j}^{\epsilon}} \widetilde{h}_{k_{j} k_{j}^{*}}^{N_{j} L_{j}}\left(\lambda_{j}\right) \frac{g_{k_{j}^{*}}(\omega)}{\left[k_{j}^{*}\right]^{\frac{\alpha}{2}}},
$$

since the contribution coming from $\mathbf{1}_{\left|k_{j}-k_{j}^{*}\right|>L_{j} N_{j}^{\epsilon}}$ is bounded by

$$
N_{j}^{-\kappa \epsilon}\left\|\left\langle\lambda_{j}\right\rangle^{b}\left\langle\frac{\left|k_{j}-k_{j}^{*}\right|}{L_{j}}\right\rangle^{\kappa} \widetilde{h}_{k_{j} k_{j}^{*}}^{N_{j}, L_{j}}\left(\lambda_{j}\right)\right\|_{L_{\lambda_{j}}^{2} l_{k_{j}, k_{j}^{*}}^{2}}
$$

which by 3.16$)$ is much smaller than the main contribution ${ }^{9}$ from $\mathbf{1}_{\left|k_{j}-k_{j}^{*}\right| \leq L_{j} N_{j}^{\epsilon}}$, provided that $\epsilon^{10}<\kappa^{-1}$. In particular, we may assume that $\left|k_{j}\right| \sim\left|k_{j}^{*}\right| \sim N_{j}$. Recall that type (C) terms satisfy (3.10), (3.11), (3.12). Slightly different from previous sections, we will denote by $w_{j}^{\left(r_{j}\right)}\left(\lambda_{j}, k_{j}\right)=\left\langle\lambda_{j}\right\rangle^{\frac{2 b_{0}}{r_{j}^{\prime}}} \widetilde{v}_{j}\left(\lambda_{j}, k_{j}\right)$ for $r_{j} \in\{2, q\}$. In order to clean the exposition, we introduce the following notations:

$$
\Upsilon_{L_{2}, L_{3}}^{N_{1}, N_{2}, N_{3}}\left(r_{2}, r_{3}\right):=\left\|\sum_{\substack{k_{1}, k_{2}, k_{3}, k,\left|k_{1}\right| \sim N_{1}, k_{3} \\\left(k_{1}, k_{2}, k_{3}\right) \in \Gamma(k) \\\left|k_{j}\right| \leq N_{j}, j=2,3}} S_{k_{1}, k_{2}, k_{3}} y_{k, k_{1}}^{0}\left(\lambda, \lambda_{1}\right) \bar{w}_{2}^{\left(r_{2}\right)}\left(\lambda_{2}, k_{2}\right) w_{3}^{\left(r_{3}\right)}\left(\lambda_{3}, k_{3}\right)\right\|_{L_{\lambda, \lambda_{1}}^{2} L_{\lambda_{2}}^{r_{2}} L_{\lambda_{3}}^{r_{3}} L_{\mu_{0} *}^{q_{0}}},
$$

and

$$
\Xi_{L_{2}, L_{3}}^{N_{1}, N_{2}, N_{3}}\left(r_{2}, r_{3}\right):=\left\|\sum_{\substack{k_{1}, k_{2}, k_{3}, k,\left|k_{1}\right| \sim N_{1} \\\left(k_{1}, k_{2}, k_{3}\right) \in \Gamma(k) \\\left|k_{j}\right| \leq N_{j}, j=2,3}} S_{k_{1}, k_{2}, k_{3}} y_{k, k_{1}}^{0}\left(\lambda, \lambda_{1}\right) \bar{w}_{2}^{\left(r_{2}\right)}\left(\lambda_{2}, k_{2}\right) w_{3}^{\left(r_{3}\right)}\left(\lambda_{3}, k_{3}\right)\right\|_{L_{\lambda}^{q^{\prime}} L_{\lambda_{1}}^{2} L_{\lambda_{2}}^{r_{2}} L_{\lambda_{3}}^{r_{3}} L_{\mu_{0} *}^{q_{0}}},
$$

where $v_{2}, v_{3}$ are of type $(\mathrm{G}),(\mathrm{C})$ or $(\mathrm{D})$ with characterized pairs $\left(N_{2}, L_{2}\right),\left(N_{3}, L_{3}\right)$, with respectively. When $v_{j}$ is of type $(\mathrm{G})$ or $(\mathrm{C})$, we have the freedom to choose $r_{j}=2$ or

\footnotetext{
${ }^{9}$ The far-diagonal part $\left|k_{j}-k_{j}^{*}\right|>L_{j} N_{j}^{\epsilon}$ can be easily treated by the deterministic estimate 77.6$)$. However we need to be cautious when $v_{2}$ and $v_{3}$ are both of type $(\mathrm{C})$. In that case, we may slightly change the constraint $\mathbf{1}_{\left|k_{j}-k_{j}^{*}\right| \leq L_{j} N_{j}^{\epsilon}}$ by $\mathbf{1}_{\left|k_{j}-k_{j}^{*}\right| \leq L_{j}\left(N_{2} \vee N_{3}\right)^{\epsilon}}$.
} 
$r_{j}=q$, while if $v_{j}$ is of type (D) (equivalently, $L_{j}=2 L_{N_{j}}$ ), it forces $r_{j}=2$. We will fix $N_{2}, N_{3} \lesssim N_{1}^{1-\delta}$ such that $N_{2} \vee N_{3} \sim L$. According to Proposition 6.1, modulo an $N^{-A}$ error, we have

$$
\left\|\Theta_{k k_{1}}^{N_{1}, L}\left(\lambda, \lambda^{\prime}\right) \mathbf{1}_{\left|k_{1}\right| \sim N_{1}}\right\|_{Z^{b, b}} \lesssim N_{1}^{50\left(2 b_{1}-1\right)} \sum_{\substack{N_{2}, N_{3}<N_{1}^{1-\delta} \\ L_{2} \leq 2 L_{N_{2}}, L_{3} \leq 2 L_{N_{3}} \\ N_{2} \vee N_{3} \sim L}} \min \left\{\Upsilon_{L_{2}, L_{3}}^{N_{1}, N_{2}, N_{3}}\left(r_{2}, r_{3}\right):\left(r_{2}, r_{3}\right) \in I_{L_{2}}^{N_{2}} \times I_{L_{3}}^{N_{3}}\right\},
$$

$$
\left\|\Theta_{k k_{1}}^{N_{1}, L}\left(\lambda, \lambda^{\prime}\right) \mathbf{1}_{\left|k_{1}\right| \sim N_{1}}\right\|_{S^{b, b, q}} \lesssim N_{1}^{50\left(2 b_{1}-1\right)} \sum_{\substack{N_{2}, N_{3}<N_{1}^{1-\delta} \\ L_{2} \leq 2 L_{N_{2}}, L_{3} \leq 2 L_{N_{3}} \\ N_{2} \vee N_{3} \sim L}} \min \left\{\Xi_{L_{2}, L_{3}}^{N_{1}, N_{2}, N_{3}}\left(r_{2}, r_{3}\right):\left(r_{2}, r_{3}\right) \in I_{L_{2}}^{N_{2}} \times I_{L_{3}}^{N_{3}}\right\},
$$

where the index set $I_{L_{j}}^{N_{j}}=\{2, q\}$ if $L_{j} \leq L_{N_{j}}$ (type (G) or (C)) and $I_{L_{j}}^{N_{j}}=\{2\}$ if $L_{j}=2 L_{N_{j}}$ (type (D))

7.2. Algorithms and reductions. In order to clean up the arguments and to emphasize the point, we describe several algorithms to be used that reduce the analysis to the multilinear summation.

Several algorithms to estimate the sum of the multi-linear expression

$$
\mathcal{M}_{N_{1}, N_{2}, N_{3}}\left(y ; a_{2}, a_{3}\right):=\left|\sum_{\substack{k_{1}, k_{2}, k_{3}, k,\left|k_{1}\right| \sim N_{1} \\\left(k_{1}, k_{2}, k_{3}\right) \in \Gamma(k) \\\left|k_{j}\right| \leq N_{j}, j=2,3}} y_{k, k_{1}} S_{k_{1}, k_{2}, k_{3}} a_{2}\left(k_{2}\right) a_{3}\left(k_{3}\right)\right|
$$

are at our proposal:

\section{-Algorithm A1: Deterministic estimates}

We may assume that $N_{3} \leq N_{2}$. Using Cauchy-Schwartz, we have

$$
\begin{aligned}
\mathcal{M}_{N_{1}, N_{2}, N_{3}}\left(y ; a_{2}, a_{3}\right) & \leq\left\|y_{k_{1}-k_{2}+k_{3}, k_{1}} a_{3}\left(k_{3}\right)\right\|_{l_{k_{1}, k_{2}, k_{3}}^{2}}\left\|a_{2}\left(k_{2}\right) S_{k_{1}, k_{2}, k_{3}}\right\|_{l_{k_{1}, k_{2}, k_{3}}^{2}} \\
& \leq\left\|y_{k, k_{1}}\right\|_{l_{k, k_{1}}^{2}}\left\|a_{3}\right\|_{l_{k_{3}}^{2}}\left\|\left|a_{2}\left(k_{2}\right)\right|^{2} \sum_{\left|k_{1}\right| \sim N_{1}} S_{k_{1}, k_{2}, k_{3}}\right\|_{l_{k_{2}, k_{3}}^{1}}^{\frac{1}{2}} \\
& \lesssim N_{1}^{\epsilon}\left(N_{1}^{1-\frac{\alpha}{2}}+N_{3}^{\frac{1}{2}}\right)\left\|y_{k, k_{1}}\right\|_{l_{k, k_{1}}^{2}}\left\|a_{2}\right\|_{l_{k_{2}}^{2}}\left\|a_{3}\right\|_{l_{k_{3}}^{2}},
\end{aligned}
$$

where we use the counting estimate

$$
\sum_{k_{1}} S_{k_{1}, k_{2}, k_{3}} \lesssim N_{1}^{\epsilon}\left(1+\frac{N_{1}^{2-\alpha}}{\left\langle k_{2}-k_{3}\right\rangle}\right)
$$

for fixed $k_{2}, k_{3}$ such that $\left|k_{2}\right| \leq N_{2} \ll N_{1},\left|k_{3}\right| \leq N_{3} \ll N_{1}$. Alternatively, we have

$$
\begin{aligned}
\mathcal{M}_{N_{1}, N_{2}, N_{3}}\left(y ; a_{2}, a_{3}\right) & \leq\left\|y_{k_{1}-k_{2}+k_{3}, k_{1}} a_{3}\left(k_{3}\right)\right\|_{l_{k_{1}, k_{2}, k_{3}}^{2}}\left\|a_{2}\left(k_{2}\right) S_{k_{1}, k_{2}, k_{3}}\right\|_{l_{k_{1}, k_{2}, k_{3}}^{2}} \\
& \leq\left\|y_{k, k_{1}}\right\|_{l_{k, k_{1}}^{2}}\left\|a_{3}\right\|_{l_{k_{3}}^{2}}\left\|a_{2}\right\|_{l_{k_{2}}^{q}}\left\|S_{k_{1}, k_{2}, k_{3}}\right\|_{l_{k_{3}}^{2} l_{k_{2}}^{\frac{2 q}{q-2}} l_{k_{1}}^{2}} \\
& \lesssim N_{1}^{\epsilon} N_{3}^{\frac{1}{2}}\left(N_{2}^{\frac{1}{2}-\frac{1}{q}}+N_{1}^{1-\frac{\alpha}{2}}\right)\left\|y_{k, k_{1}}\right\|_{l_{k, k_{1}}^{2}}\left\|a_{3}\right\|_{l_{k_{3}}^{2}}\left\|a_{2}\right\|_{l_{k_{2}}^{q}} .
\end{aligned}
$$

Choosing in particular $\epsilon=\kappa^{-0.01}$, replacing $y_{k k_{1}}, a_{2}, a_{3}$ by $y_{k k_{1}}^{0}\left(\lambda, \lambda_{1}\right), w_{2}^{(2)}\left(\lambda_{2}, k_{2}\right), w_{3}^{(2)}\left(\lambda_{3}, k_{3}\right)$ or $y_{k k_{1}}^{0}\left(\lambda, \lambda_{1}\right), w_{2}^{(q)}\left(\lambda_{2}, k_{2}\right), w_{3}^{(2)}\left(\lambda_{3}, k_{3}\right)$ with respectively and then taking $L_{\lambda, \lambda_{1}}^{2} L_{\lambda_{2}}^{2} L_{\lambda_{3}}^{2} L_{\mu_{0} *}^{q_{0}}$ or $L_{\lambda, \lambda_{1}}^{2} L_{\lambda_{3}}^{2} L_{\lambda_{2}}^{q} L_{\mu_{0} *}^{q_{0}}$, we obtain (note that $\alpha q_{0}^{-1}<\kappa^{-0.09}$ ): 
Lemma 7.1. Assume that $N_{1} \gg N_{2}, N_{3}$, then we have

$(7.6)$

$$
\Upsilon_{L_{2}, L_{3}}^{N_{1}, N_{2}, N_{3}}(2,2) \lesssim N_{1}^{\kappa^{-0.1}}\left(N_{1}^{1-\frac{\alpha}{2}}+\left(N_{2} \wedge N_{3}\right)^{\frac{1}{2}}\right)\left\|y_{k, k_{1}}^{0}\left(\lambda, \lambda_{1}\right)\right\|_{L_{\lambda, \lambda_{1}}^{2} l_{k, k_{1}}^{2}}\left\|v_{2}\right\|_{X^{0, b_{0}}}\left\|v_{3}\right\|_{X^{0, b_{0}}} .
$$

Moreover, if $N_{3} \leq N_{2}$, we have alternatively

$$
\Upsilon_{L_{2}, L_{3}}^{N_{1}, N_{2}, N_{3}}(q, 2) \lesssim N_{1}^{\kappa^{-0.1}} N_{3}^{\frac{1}{2}}\left(N_{2}^{\frac{1}{2}}+N_{2}^{\frac{1}{q}} N_{1}^{1-\frac{\alpha}{2}}\right)\left\|y_{k k_{1}}^{0}\left(\lambda, \lambda_{1}\right)\right\|_{L_{\lambda, \lambda_{1}}^{2} l_{k, k_{1}}^{2}}\left\|v_{2}\right\|_{\substack{X_{\infty, q}^{0}, \frac{2 b_{0}}{q^{\prime}} \\ \|}}\left\|v_{3}\right\|_{X^{0, b_{0}}}
$$

if $N_{2} \leq N_{3}$,

$$
\Upsilon_{L_{2}, L_{3}}^{N_{1}, N_{2}, N_{3}}(2, q) \lesssim N_{1}^{\kappa^{-0.1}} N_{2}^{\frac{1}{2}}\left(N_{3}^{\frac{1}{2}}+N_{3}^{\frac{1}{q}} N_{1}^{1-\frac{\alpha}{2}}\right)\left\|y_{k k_{1}}^{0}\left(\lambda, \lambda_{1}\right)\right\|_{L_{\lambda, \lambda_{1}}^{2} l_{k, k_{1}}^{2}}\left\|v_{2}\right\|_{X^{0, b_{0}}}\left\|v_{3}\right\|_{\substack{0, \frac{2 b_{0}}{q^{\prime}} \\ X_{\infty}}} .
$$

Remark 7.2. We will use (7.6) when $v_{2}, v_{3}$ are of type $(D)$ or type $(C)$ with large $L_{j}$. The estimate (7.7) or (7.8) is useful when $v_{2}, v_{3}$ are all of type $(G)$ or $(C)$ and the $L_{j}$ parameter of one type $(C)$ term is greater than the $N_{j}$ parameter of the other. Note that this is the case where we are no not able to exploit Wiener chaos.

Similarly, we have

$$
\begin{aligned}
\mathcal{M}_{N_{1}, N_{2}, N_{3}}\left(y ; a_{2}, a_{3}\right) & \leq\left\|y_{k, k+k_{2}-k_{3}} a_{3}\left(k_{3}\right)\right\|_{l_{k}^{1} l_{k_{2}, k_{3}}^{2}}\left\|a_{2}\left(k_{2}\right) S_{k+k_{2}-k_{3}, k_{2}, k_{3}}\right\|_{l_{k}^{\infty} l_{k_{2}, k_{3}}^{2}} \\
& \lesssim\left\|y_{k, k_{1}}\right\|_{l_{k}^{1} l_{k_{1}}^{2}}\left\|a_{2}\right\|_{l_{k_{2}}^{2}}\left\|a_{3}\right\|_{l_{k_{3}}^{2}},
\end{aligned}
$$

where we use the counting estimate

$$
\sup _{\left|k_{2}\right| \leq N_{2}} \sum_{\left|k_{3}\right| \leq N_{3}} S_{k+k_{2}-k_{3}, k_{2}, k_{3}} \lesssim 1
$$

for fixed $k$, since $|k| \sim N_{1} \gg N_{2}, N_{3}$. Alternatively, when $N_{3} \leq N_{2}$, we have

$$
\begin{aligned}
\mathcal{M}_{N_{1}, N_{2}, N_{3}}\left(y ; a_{2}, a_{3}\right) & \leq\left\|y_{k, k+k_{2}-k_{3}} a_{3}\left(k_{3}\right)\right\|_{l_{k}^{1} l_{k_{2}, k_{3}}^{2}}\left\|a_{2}\left(k_{2}\right) S_{k+k_{2}-k_{3}, k_{2}, k_{3}}\right\|_{l_{k}^{\infty} l_{k_{2}, k_{3}}^{2}} \\
& \leq\left\|y_{k k_{1}}\right\|_{l_{k}^{1} l_{k_{1}}^{2}}\left\|a_{3}\left(k_{3}\right)\right\|_{l_{k_{3}}^{2}}\left\|a_{2}\right\|_{l_{k_{2}}^{q}}\left\|S_{k+k_{2}-k_{3}, k_{2}, k_{3}}\right\|_{l_{k_{3}}^{2} l_{k_{2}}^{\frac{2 q}{q-2}}} \\
& \lesssim N_{3}^{\frac{1}{2}}\left\|y_{k k_{1}}\right\|_{l_{k}^{1} l_{k_{1}}^{2}}\left\|a_{3}\left(k_{3}\right)\right\|_{l_{k_{3}}^{2}}\left\|a_{2}\right\|_{l_{k_{2}}^{q}} \cdot
\end{aligned}
$$

Substituting the bounds (7.5), (7.9) with $y_{k, k_{1}}=y_{k, k_{1}}^{0}\left(\lambda, \lambda_{1}\right), a_{2}\left(k_{2}\right)=w_{2}^{\left(r_{2}\right)}\left(\lambda_{2}, k_{2}\right)$, and $a_{3}\left(k_{3}\right)=w_{3}^{\left(r_{3}\right)}\left(\lambda_{3}, k_{3}\right)$ and then taking $L_{\lambda}^{q^{\prime}} L_{\lambda_{1}}^{2} L_{\lambda_{2}}^{2} L_{\lambda_{3}}^{2} L_{\mu_{0} *}^{q_{0}}$ norm or $L_{\lambda}^{q^{\prime}} L_{\lambda_{1}}^{2} L_{\lambda_{2}}^{q} L_{\lambda_{3}}^{2} L_{\mu_{0} *}^{q_{0}}$ (after switching the order of $L^{p}$ spaces by Minkowski if necessary), we have proved:

Lemma 7.3. Assume that $N_{1} \gg N_{2}, N_{3}$, then we have

$$
\Xi_{L_{2}, L_{3}}^{N_{1}, N_{2}, N_{3}}(2,2) \lesssim\left\|y_{k, k_{1}}^{0}\left(\lambda, \lambda_{1}\right)\right\|_{l_{k}^{1} L_{\lambda}^{q^{\prime}} L_{\lambda_{1}}^{2} l_{k_{1}}^{2}}\left\|v_{2}\right\|_{X^{0, b_{0}}}\left\|v_{3}\right\|_{X^{0, b_{0}}}
$$

Alternatively, when $N_{3} \leq N_{2}$ we have

$$
\Xi_{L_{2}, L_{3}}^{N_{1}, N_{2}, N_{3}}(q, 2) \lesssim N_{1}^{\kappa^{-0.1}} N_{2}^{\frac{1}{q}} N_{3}^{\frac{1}{2}}\left\|y_{k, k_{1}}^{0}\left(\lambda, \lambda_{1}\right)\right\|_{l_{k}^{1} L_{\lambda}^{q^{\prime}} L_{\lambda_{1}}^{2} l_{k_{1}}^{2}}\left\|v_{2}\right\|_{\substack{X_{\infty}, q \\ q^{\prime}}}\left\|v_{3}\right\|_{X^{0, b_{0}}},
$$

and when $N_{2} \leq N_{3}$, we have

$$
\Xi_{L_{2}, L_{3}}^{N_{1}, N_{2}, N_{3}}(2, q) \lesssim N_{1}^{\kappa^{-0.1}} N_{3}^{\frac{1}{q}} N_{2}^{\frac{1}{2}}\left\|y_{k, k_{1}}^{0}\left(\lambda, \lambda_{1}\right)\right\|_{l_{k}^{1} L_{\lambda}^{q^{\prime}} L_{\lambda_{1}}^{2} l_{k_{1}}^{2}}\left\|v_{2}\right\|_{X^{0, b_{0}}}\left\|v_{3}\right\|_{X_{\infty, q}^{0, \frac{2 b_{0}}{q^{\prime}}}} .
$$

\section{-Algorithm A2: One random input}

Recall that $w_{j}^{\left(r_{j}\right)}\left(\lambda_{j}, k_{j}\right)=\left\langle\lambda_{j}\right\rangle^{\frac{2 b_{0}}{r_{j}^{\prime}}} \widetilde{v}_{j}\left(\lambda_{j}, k_{j}\right)$. According to $v_{2}$ is of type $(\mathrm{C}),(\mathrm{G})$ or $v_{3}$ is of type (C), (G), for fixed $\lambda, \lambda_{1}, \lambda_{2}, \lambda_{3}, \mu_{0}$, we have 
Lemma 7.4. We have

$\mathcal{M}_{N_{1}, N_{2}, N_{3}}\left(y ; w_{2}^{(2)}, w_{3}^{(q)}\right) \leq\left[\sup _{k_{2}}\left|\sigma_{k_{2} k_{2}}^{(3)}\right|+\left(\sum_{k_{2}, k_{2}^{\prime}: k_{2} \neq k_{2}^{\prime}}\left|\sigma_{k_{2} k_{2}^{\prime}}^{(3)}\right|^{2}\right)^{\frac{1}{2}}\right]^{\frac{1}{2}}\left\|y_{k, k_{1}}^{0}\left(\lambda, \lambda_{1}\right)\right\|_{l_{k_{1}, k}^{2}}\left\|w_{2}\left(\lambda_{2}, k_{2}\right)\right\|_{l_{k_{2}}^{2}}$,

$\mathcal{M}_{N_{1}, N_{2}, N_{3}}\left(y ; w_{2}^{(q)}, w_{3}^{(2)}\right) \leq\left[\sup _{k_{3}}\left|\sigma_{k_{3} k_{3}}^{(2)}\right|+\left(\sum_{k_{3}, k_{3}^{\prime}: k_{3} \neq k_{3}^{\prime}}\left|\sigma_{k_{3} k_{3}^{\prime}}^{(2)}\right|^{2}\right)^{\frac{1}{2}}\right]^{\frac{1}{2}}\left\|y_{k, k_{1}}^{0}\left(\lambda, \lambda_{1}\right)\right\|_{l_{k_{1}, k}^{2}}\left\|w_{3}\left(\lambda_{3}, k_{3}\right)\right\|_{l^{2}}$, where

$$
\sigma_{k_{2} k_{2}^{\prime}}^{(3)}=\sum_{\substack{k_{1}, k: k_{1} \neq k,\left|k_{1}\right| \sim k \mid \sim N_{1}}} w_{3}^{(q)}\left(\lambda_{3}, k+k_{2}-k_{1}\right) \overline{w_{3}^{(q)}}\left(\lambda_{3}, k+k_{2}^{\prime}-k_{1}\right) S_{k_{1}, k_{2}, k+k_{2}-k_{1}} S_{k_{1}, k_{2}^{\prime}, k+k_{2}^{\prime}-k_{1}},
$$

$$
\sigma_{k_{3} k_{3}^{\prime}}^{(2)}=\sum_{\substack{k_{1}, k: k_{1} \neq k,\left|k_{1}\right| \sim|k| \sim N_{1}}} \overline{w_{2}^{(q)}}\left(\lambda_{2}, k_{1}+k_{3}-k\right) w_{2}^{(q)}\left(\lambda_{2}, k_{1}+k_{3}^{\prime}-k\right) S_{k_{1}, k_{1}+k_{3}-k, k_{3}} S_{k_{1}, k_{1}+k_{3}^{\prime}-k, k_{3}^{\prime}},
$$

Note that the matrix elements $\sigma_{k_{j} k_{j}^{\prime}}^{(j)}$ are functions of $\lambda_{j}, \mu_{0}$.

Proof. We only prove 7.13 since the proof for 7.14 is similar. Consider the operator (depending on $\lambda_{2}, \mu_{0}$ ):

$$
\mathcal{G}_{2}: l_{k, k_{1}}^{2} \rightarrow l_{k_{2}}^{2}: y_{k, k_{1}} \mapsto \sum_{k_{1}, k} y_{k, k_{1}} \widetilde{w}_{3}\left(\lambda_{3}, k+k_{2}-k_{1}\right) S_{k_{1}, k_{2}, k+k_{2}-k_{1}},
$$

where in the summation, we do not display the constraints $k_{1} \neq k,\left|k_{1}\right| \sim|k| \sim N$. By Cauchy-Schwartz,

$$
\begin{aligned}
\mathcal{M}_{N_{1}, N_{2}, N_{3}}\left(y ; w_{2}^{(2)}, w_{3}^{(q)}\right) & =\left|\sum_{k_{2}} \mathcal{G}_{2}\left(y^{0}\right)\left(k_{2}\right) \widetilde{\widetilde{w}_{2}}\left(k_{2}\right)\right|=\left|\left(\mathcal{G}_{2}\left(y^{0}\right), \widetilde{w}_{2}\right)_{l^{2}}\right| \\
& \leq\left\|\mathcal{G}_{2}\right\|_{l_{k_{1}, k}^{2} \rightarrow l_{k_{2}}^{2}}\left\|y_{k_{1}, k}^{0}\right\|_{l_{k_{1}, k}^{2}}^{2}\left\|\widetilde{w}_{2}\right\|_{l_{k_{2}}^{2}} .
\end{aligned}
$$

Note that $\left\|\mathcal{G}_{2}\right\|_{l_{k_{1}, k}^{2} \rightarrow l_{k_{2}}^{2}}^{2}=\left\|\mathcal{G}_{2} \mathcal{G}_{2}^{*}\right\|_{l_{k_{2}}^{2} \rightarrow l_{k_{2}}^{2}}$, and one verifies that matrix element of $\mathcal{G}_{2} \mathcal{G}_{2}^{*}$ is exactly $\sigma_{k_{2} k_{2}^{\prime}}^{(2)}$. Hence by Lemma 2.11 , we obtain 7.13 . The proof of Lemma 7.4 is complete.

Denote by $h_{k_{j} k_{j}^{*}}^{N_{j} L_{j}\left(r_{j}\right)}\left(\lambda_{j}, k_{j}\right)=\left\langle\lambda_{j}\right\rangle^{\frac{2 b_{0}}{r_{j}^{\prime}}} \widetilde{h}_{k_{j} k_{j}^{*}}^{N_{j} L_{j}}\left(\lambda_{j}, k_{j}\right)$, and when there is no risk of confusing, we will denote simply by $h_{k_{j} k_{j}^{*}}^{\left(r_{j}\right)}\left(\lambda_{j}, k_{j}\right)$. When $v_{3}$ is of type $(\mathrm{G})$ or $(\mathrm{C})$, we have

$$
\begin{aligned}
& \Upsilon_{L_{2}, L_{3}}^{N_{1}, N_{2}, N_{3}}(2, q) \\
\leq & \left(\left\|\sigma_{k_{2} k_{2}}^{(3)}\left(\lambda_{3}, \mu_{0}\right)\right\|_{L_{\lambda_{3}}^{\frac{q}{2}} \frac{q_{\mu_{0}} L_{k_{2}}}{2}}^{\frac{q_{0}}{2}}+\left\|\sigma_{k_{2} k_{2}^{\prime}}^{(3)}\left(\lambda_{3}, \mu_{0}\right) \mathbf{1}_{k_{2} \neq k_{2}^{\prime}}\right\|_{L_{\lambda_{3}}^{\frac{q}{2}} \frac{q_{\mu_{0} *}}{2} l_{k_{2}, k_{2}^{\prime}}^{2}}^{2}\right)\left\|y_{k k_{1}}^{0}\left(\lambda, \lambda_{1}\right)\right\|_{L_{\lambda, \lambda_{1}}^{2} l_{k, k_{1}}^{2}}\left\|v_{2}\right\|_{X^{0, b_{0}}}
\end{aligned}
$$

and when $v_{2}$ is of type $(\mathrm{G})$ or $(\mathrm{C})$, we have

$$
\begin{aligned}
& \Upsilon_{L_{2}, L_{3}}^{N_{1}, N_{2}, N_{3}}(q, 2) \\
\leq & \left(\left\|\sigma_{k_{3} k_{3}}^{(2)}\left(\lambda_{2}, \mu_{0}\right)\right\|_{L_{\lambda_{2}}^{\frac{q}{2}} L_{\mu_{0} *}^{\frac{q_{0}}{2}} l_{k_{3}}}^{\frac{1}{2}}+\left\|\sigma_{k_{3} k_{3}^{\prime}}^{(2)}\left(\lambda_{2}, \mu_{0}\right) \mathbf{1}_{k_{3} \neq k_{3}^{\prime}}\right\|_{L_{\lambda_{2}}^{\frac{q}{2}} L_{\mu_{0} *}^{\frac{q_{0}}{2}} l_{k_{3}, k_{3}^{\prime}}^{2}}^{\frac{1}{2}}\right)\left\|y_{k k_{1}}^{0}\left(\lambda, \lambda_{1}\right)\right\|_{L_{\lambda, \lambda_{1}}^{2} l_{k, k_{1}}^{2}}\left\|v_{3}\right\|_{X^{0, b_{0}}} .
\end{aligned}
$$


Note that

$$
\begin{aligned}
& \sigma_{k_{2} k_{2}^{\prime}}^{(3)}=\sum_{\substack{k_{1}, k: k_{1} \neq k \\
\left|k_{1}\right| \sim \sim k \mid \sim N_{1}}} S_{k_{1}, k_{2}, k+k_{2}-k_{1}} S_{k_{1}, k_{2}^{\prime}, k+k_{2}^{\prime}-k_{1}} \sum_{\substack{\left|k_{3}^{*}\right|,\left|k_{3}^{\prime *}\right| \sim N_{3} \\
\left|k_{3}-k_{3}^{*}\right| \leq L_{3} N_{3}^{\epsilon} \\
\left|k_{3}^{\prime}-k_{3}^{\prime *}\right| \leq L_{3} N_{3}^{\epsilon}}} h_{k+k_{2}-k_{1}, k_{3}^{*}}^{(q)}\left(\lambda_{3}\right) \bar{h}_{k+k_{2}^{\prime}-k_{1}, k_{3}^{* *}}^{(q)}\left(\lambda_{3}\right) \frac{g_{k_{3}^{*}} \bar{g}_{k_{3}^{\prime *}}}{\left[k_{3}^{*}\right]^{\frac{\alpha}{2}}\left[k_{3}^{*}\right]^{\frac{\alpha}{2}}},
\end{aligned}
$$

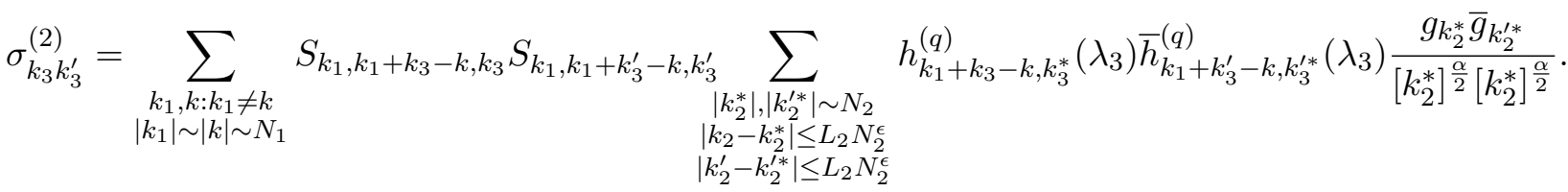

If we are able to establish the estimates:

$$
\sup _{\left|k_{2}\right| \sim N_{2}}\left(\mathbb{E}^{\mathcal{C}_{3}}\left[\left|\sigma_{k_{2} k_{2}}^{(3)}\right|^{2}\right]\right)^{\frac{1}{2}}+\left(\sum_{k_{2} \neq k_{2}^{\prime}} \mathbb{E}^{\mathcal{C}_{3}}\left[\left|\sigma_{k_{2} k_{2}^{\prime}}^{(3)}\right|^{2}\right]\right)^{\frac{1}{2}} \leq \Lambda_{3}\left(N_{1}, N_{2}, N_{3}, L_{2}, L_{3}\right)\left\|h_{k_{3} k_{3}^{*}}^{(q)}\left(\lambda_{3}\right)\right\|_{l_{k_{3}} l_{k_{3}^{*}}^{2}}^{2},
$$

and

$$
\sup _{\left|k_{3}\right| \sim N_{3}}\left(\mathbb{E}^{\mathcal{C}_{2}}\left[\left|\sigma_{k_{3} k_{3}}^{(2)}\right|^{2}\right]\right)^{\frac{1}{2}}+\left(\sum_{k_{3} \neq k_{3}^{\prime}} \mathbb{E}^{\mathcal{C}_{2}}\left[\left|\sigma_{k_{3} k_{3}^{\prime}}^{(2)}\right|^{2}\right]\right)^{\frac{1}{2}} \leq \Lambda_{2}\left(N_{1}, N_{2}, N_{3}, L_{2}, L_{3}\right)\left\|h_{k_{2} k_{2}^{*}}^{(q)}\left(\lambda_{2}\right)\right\|_{l_{k_{2}}^{\infty} l_{k_{2}^{*}}^{2}}^{2},
$$

then from the estimate

$$
\left\|h_{k_{j} k_{j}^{*}}^{(q)}\left(\lambda_{j}\right)\right\|_{L_{\lambda_{j}}^{q} l_{k_{j}}^{\infty} l_{k_{j}^{*}}^{2}} \leq\left\|h_{k_{j} k_{j}^{*}}^{(q)}\left(\lambda_{j}\right)\right\|_{L_{\lambda_{j}}^{q} l_{k_{j}}^{q} l_{k_{j}^{*}}^{2}} \lesssim N_{j}^{\frac{1}{q}}\left\|h_{k_{j} k_{j}^{*}}^{(q)}\left(\lambda_{j}\right)\right\|_{l_{k_{j}}^{\infty} L_{\lambda_{j}}^{q} l_{k_{j}^{*}}^{2}}
$$

and the large deviation property (Corollary 2.17), we deduce that outside a set of probability $<\mathrm{e}^{-N_{1}^{\theta} R}$ :

$\Upsilon_{L_{2}, L_{3}}^{N_{1}, N_{2}, N_{3}}(2, q) \lesssim R N_{1}^{\frac{\alpha}{q_{0}}+\theta} N_{3}^{\frac{1}{q}} \Lambda_{3}\left(N_{1}, N_{2}, N_{3}, L_{2}, L_{3}\right)^{\frac{1}{2}}\left\|y_{k k_{1}}^{0}\left(\lambda, \lambda_{1}\right)\right\|_{L_{\lambda, \lambda_{1}}^{2} l_{k, k_{1}}^{2}}\left\|v_{2}\right\|_{X^{0, b_{0}}}\left\|h^{N_{3} L_{3}}\right\|_{S^{b_{0}, q},}$

and

$\Upsilon_{L_{2}, L_{3}}^{N_{1}, N_{2}, N_{3}}(q, 2) \lesssim N_{1}^{\frac{\alpha}{q_{0}}+\theta} N_{2}^{\frac{1}{q}} \Lambda_{2}\left(N_{1}, N_{2}, N_{3}, L_{2}, L_{3}\right)^{\frac{1}{2}}\left\|y_{k k_{1}}^{0}\left(\lambda, \lambda_{1}\right)\right\|_{L_{\lambda, \lambda_{1}}^{2} l_{k, k_{1}}^{2}}\left\|v_{3}\right\|_{X^{0, b_{0}}}\left\|h^{N_{2} L_{2}}\right\|_{S^{b_{0}, q}}$.

The algorithm to estimate $\Xi_{L_{2}, L_{3}}^{N_{1}, N_{2}, N_{3}}(2, q)$ and $\Xi_{L_{2}, L_{3}}^{N_{1}, N_{2}, N_{3}}(2, q)$ is similar. Denote by

$$
\sigma_{k, k_{1} ; k_{2}}^{(3)}=\sum_{\substack{k_{3}^{*}:\left|k_{3}^{*}\right| \sim N_{3} \\\left|k_{3}-k_{3}^{*}\right| \leq L_{3} N_{3}^{\epsilon}}} h_{k+k_{2}-k_{1}, k_{3}^{*}}^{(q)}\left(\lambda_{3}\right) \frac{g_{k_{3}^{*}}}{\left[k_{3}^{*}\right]^{\frac{\alpha}{2}}} S_{k_{1}, k_{2}, k+k_{2}-k_{1}}
$$

and

$$
\sigma_{k, k_{1} ; k_{3}}^{(2)}=\sum_{\substack{k_{2}^{*}:\left|k_{2}^{*}\right| \sim N_{2} \\\left|k_{2}-k_{2}^{*}\right| \leq L_{2} N_{2}^{\epsilon}}} h_{k_{1}+k_{3}-k, k_{2}^{*}}^{(q)}\left(\lambda_{2}\right) \frac{g_{k_{2}^{*}}^{*}}{\left[k_{2}^{*}\right]^{\frac{\alpha}{2}}} S_{k_{1}, k_{1}+k_{3}-k, k_{3}} .
$$

Then by Lemma 2.13 and Corollary 2.17 , if we are able to establish the estimates:

$$
\begin{aligned}
& \sup _{k, k_{1}} \sum_{k_{2}} \mathbb{E}^{\mathcal{C}_{3}}\left[\left|\sigma_{k, k_{1} ; k_{2}}^{(3)}\right|^{2}\right]+\sup _{k, k^{\prime}:|k|,\left|k^{\prime}\right| \sim N_{1}}\left(\sum_{\substack{k_{1}, k_{1}^{\prime} \\
\left(k, k_{1}\right) \neq\left(k^{\prime}, k_{1}^{\prime}\right)}} \mathbb{E}^{\mathcal{C}_{3}}\left[\left|\sum_{k_{2}} \sigma_{k^{\prime}, k_{1}^{\prime} ; k_{2}}^{(3)} \bar{\sigma}_{k, k_{1} ; k_{2}}^{(3)}\right|^{2}\right]\right)^{\frac{1}{2}} \\
\leq & \widetilde{\Lambda}_{3}\left(N_{1}, N_{2}, N_{3}, L_{2}, L_{3}\right)\left\|h_{k_{3} k_{3}^{*}}^{(q)}\left(\lambda_{3}\right)\right\|_{l_{k_{3}}^{\infty} l_{k_{3}^{*}}^{2}}^{2},
\end{aligned}
$$


and

$$
\begin{aligned}
& \quad \sup _{k, k_{1}} \sum_{k_{3}} \mathbb{E}^{\mathcal{C}_{2}}\left[\left|\sigma_{k, k_{1} ; k_{3}}^{(2)}\right|^{2}\right]+\sup _{k, k^{\prime}:|k|,\left|k^{\prime}\right| \sim N_{1}}\left(\sum_{\substack{k_{1}, k_{1}^{\prime} \\
\left(k, k_{1}\right) \neq\left(k^{\prime}, k_{1}^{\prime}\right)}} \mathbb{E}^{\mathcal{C}_{2}}\left[\left|\sum_{k_{3}} \sigma_{k^{\prime}, k_{1}^{\prime} ; k_{3}}^{(2)} \bar{\sigma}_{k, k_{1} ; k_{3}}^{(2)}\right|^{2}\right]\right)^{\frac{1}{2}} \\
& \leq \widetilde{\Lambda}_{2}\left(N_{1}, N_{2}, N_{3}, L_{2}, L_{3}\right)\left\|h_{k_{2} k_{2}^{*}}^{(q)}\left(\lambda_{2}\right)\right\|_{l_{k_{2}}^{\infty} l_{k_{2}^{*}}^{2}}^{2}
\end{aligned}
$$

then outside a set of probability $<\mathrm{e}^{-N_{1}^{\theta} R}$, we have

$\Xi_{L_{2}, L_{3}}^{N_{1}, N_{2}, N_{3}}(2, q) \lesssim R N_{1}^{\frac{\alpha}{q_{0}}+\theta} N_{3}^{\frac{1}{q}} \widetilde{\Lambda}_{3}\left(N_{1}, N_{2}, N_{3}, L_{2}, L_{3}\right)^{\frac{1}{2}}\left\|y_{k k_{1}}^{0}\left(\lambda, \lambda_{1}\right)\right\|_{l_{k}^{1} L_{\lambda}^{q^{\prime}} L_{\lambda_{1}}^{2} l_{k_{1}}^{2}}\left\|v_{2}\right\|_{X^{0, b_{0}}}\left\|h^{N_{3} L_{3}}\right\|_{S^{b_{0}, q}}$ and

$$
\Xi_{L_{2}, L_{3}}^{N_{1}, N_{2}, N_{3}}(q, 2) \lesssim R N_{1}^{\frac{\alpha}{q_{0}}+\theta} N_{2}^{\frac{1}{q}} \widetilde{\Lambda}_{2}\left(N_{1}, N_{2}, N_{3}, L_{2}, L_{3}\right)^{\frac{1}{2}}\left\|y_{k k_{1}}^{0}\left(\lambda, \lambda_{1}\right)\right\|_{l_{k}^{1} L_{\lambda}^{q^{\prime}} L_{\lambda_{1}}^{2} l_{k_{1}}^{2}}\left\|v_{3}\right\|_{X^{0, b_{0}}}\left\|h^{N_{2} L_{2}}\right\|_{S^{b_{0}, q}} .
$$

\section{-Algorithm A3: Two random inputs}

We only use this algorithm to deal with the case where $v_{2}$ and $v_{3}$ are both of type $(\mathrm{G})$ or (C) with characterized parameters $\left(N_{2}, L_{2}\right)$ and $\left(N_{3}, L_{3}\right)$ satisfying $\left(L_{2} \vee L_{3}\right) \ll\left(N_{2} \wedge N_{3}\right)$. Assume without loss of generality that $N_{2} \leq N_{3}$, we have

$$
\left|\Upsilon_{L_{2}, L_{3}}^{N_{1}, N_{2}, N_{3}}(2, q)\right| \leq\left\|y_{k, k_{1}}^{0}\left(\lambda, \lambda_{1}\right)\right\|_{l_{k, k_{1}}^{2}}\left\|\mathbf{1}_{k_{1} \neq k} G_{k_{1}, k}\right\|_{l_{k, k_{1}}^{2}}
$$

and

$$
\left|\Xi_{L_{2}, L_{3}}^{N_{1}, N_{2}, N_{3}}(2, q)\right| \leq\left\|y_{k, k_{1}}^{0}\left(\lambda, \lambda_{1}\right)\right\|_{l_{k}^{1} l_{k_{1}}^{2}}\left\|\mathbf{1}_{k_{1} \neq k} G_{k_{1}, k}\right\|_{l_{k}^{\infty} l_{k_{1}}^{2}},
$$

where

$$
G_{k, k_{1}}\left(\lambda_{2}, \lambda_{3}, \mu_{0}\right):=\sum_{k_{3}} S_{k_{1}, k_{1}-k+k_{3}, k_{3}} \sum_{k_{2}^{*}, k_{3}^{*}} h_{k_{1}-k+k_{3}, k_{2}^{*}}^{(q)}\left(\lambda_{2}\right) h_{k_{3} k_{3}^{*}}^{(2)}\left(\lambda_{3}\right) \frac{\bar{g}_{k_{2}^{*}} g_{k_{3}^{*}}}{\left[k_{2}^{*}\right]^{\frac{\alpha}{2}}\left[k_{3}^{*}\right]^{\frac{\alpha}{2}}}
$$

Note that $h_{k_{2} k_{2}^{*}}^{(q)}$ and $h_{k_{3} k_{3}^{*}}^{(2)}$ are $\mathcal{C}_{L_{2} \vee L_{3}}$ measurable, independent of $\left\{g_{k_{2}^{*}}(\omega):\left|k_{2}^{*}\right| \sim N_{2}\right\}$ and $\left\{g_{k_{3}^{*}}(\omega):\left|k_{3}^{*}\right| \sim N_{3}\right\}$. If we are able to show that

$$
\sum_{k_{1}, k: k_{1} \neq k} \mathbb{E}^{\mathcal{C}}\left[\left|G_{k_{1}, k}\right|^{2}\right] \leq \Lambda_{2,3}\left(N_{1}, N_{2}, N_{3}, L_{2}, L_{3}\right)\left\|h_{k_{3} k_{3}^{*}}^{(q)}\right\|_{l_{k_{3}} l_{k_{3}^{*}}^{2}}^{2}\left\|h_{k_{2} k_{2}^{*}}^{(2)}\right\|_{l_{k_{2} k_{2}^{*}}^{2}}^{2}
$$

and

$$
\sup _{|k| \sim N_{1}} \sum_{k_{1}} \mathbb{E}^{\mathcal{C}}\left[\left|G_{k_{1}, k}\right|^{2}\right] \leq \widetilde{\Lambda}_{2,3}\left(N_{1}, N_{2}, N_{3}, L_{2}, L_{3}\right)\left\|h_{k_{3} k_{3}^{*}}^{(q)}\right\|_{l_{k_{3}}^{\infty} l_{k_{3}^{*}}^{2}}^{2}\left\|h_{k_{2} k_{2}^{*}}^{(2)}\right\|_{l_{k_{2} k_{2}^{*}}^{2}}^{2}
$$

where $\mathcal{C}=\mathcal{C}_{L_{2} \vee L_{3}}$, then from Corollary 2.17 , outside a set of probability $<\mathrm{e}^{-N_{1}^{\theta} R}$, we have

$$
\left|\Upsilon_{L_{2}, L_{3}}^{N_{1}, N_{2}, N_{3}}(2, q)\right| \lesssim R N_{1}^{\frac{\alpha}{q_{0}}+\theta} N_{3}^{\frac{1}{q}} \Lambda_{2,3}\left(N_{1}, N_{2}, N_{3}, L_{2}, L_{3}\right)^{\frac{1}{2}}\left\|h^{N_{3} L_{3}}\right\|_{S^{b_{0}, q}}\left\|h^{N_{2} L_{2}}\right\|_{Z^{b_{0}}}
$$

and

$$
\left|\Xi_{L_{2}, L_{3}}^{N_{1}, N_{2}, N_{3}}(2, q)\right| \lesssim R N_{1}^{\frac{\alpha}{q_{0}}+\frac{1}{q}+\theta} N_{3}^{\frac{1}{q}} \widetilde{\Lambda}_{2,3}\left(N_{1}, N_{2}, N_{3}, L_{2}, L_{3}\right)^{\frac{1}{2}}\left\|h^{N_{3} L_{3}}\right\|_{S^{b_{0}, q}}\left\|h^{N_{2} L_{2}}\right\|_{Z^{b_{0}}} .
$$

We remark that when $v_{2}$ is of type $(\mathrm{G})$ or $v_{2}, v_{3}$ are both of type $(\mathrm{G})$ (note that $N_{2} \leq N_{3}$ ), we should instead estimate $\Upsilon_{L_{2}, L_{3}}^{N_{1}, N_{2}, N_{3}}(q, q)$ and $\Xi_{L_{2}, L_{3}}^{N_{1}, N_{2}, N_{3}}(q, q)$ and the norms on the right side of 7.28 and 7.29 should be modified by $\left\|h^{N_{2} L_{2}}\right\|_{S^{b_{0}, q}}\left\|h^{N_{3} L_{3}}\right\|_{S^{b_{0}, q}}$. 
7.3. Implementing the algorithms. In this subsection, we compute

$$
\Upsilon_{L_{2}, L_{3}}^{N_{1}, N_{2}, N_{3}} \text { and } \Xi_{L_{2}, L_{3}}^{N_{1}, N_{2}, N_{3}}
$$

where 10

$$
\Upsilon_{L_{2}, L_{3}}^{N_{1}, N_{2}, N_{3}}:=\min \left\{\Upsilon_{L_{2}, L_{3}}^{N_{1}, N_{2}, N_{3}}\left(r_{2}, r_{3}\right):\left(r_{2}, r_{3}\right) \in\{2, q\} \times\{2, q\}\right\}
$$

and

$$
\Xi_{L_{2}, L_{3}}^{N_{1}, N_{2}, N_{3}}:=\min \left\{\Xi_{L_{2}, L_{3}}^{N_{1}, N_{2}, N_{3}}\left(r_{2}, r_{3}\right):\left(r_{2}, r_{3}\right) \in\{2, q\} \times\{2, q\}\right\}
$$

for all possible $N_{1}, N_{2}, N_{3}$ such that $N_{1} \gg N_{2}, N_{3}$ and $L_{j}<N_{j}^{1-\delta}, j=2,3$. This will be done by executing the Algorithm A1-Algorithm A3 that we have just described.

\section{-Two random inputs}

We begin with the case that $v_{2}, v_{3}$ are both of type $(\mathrm{G})$ or $(\mathrm{C})$.

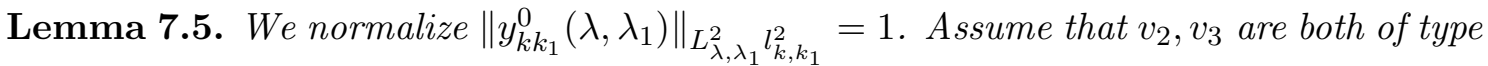
$(G)$ or $(C)$ with characterized parameters $\left(N_{2}, L_{2}\right),\left(N_{3}, L_{3}\right)$ satisfying $L_{2} \vee L_{3} \ll\left(N_{2} \wedge N_{3}\right)$. Then outside a set of probability $<\mathrm{e}^{-N_{1}^{\theta} R}$, we have:

(i) If $N_{2} \ll N_{3} \ll N_{1}$ and $v_{2}$ is of type $(C)$, then

$$
\left|\Upsilon_{L_{2}, L_{3}}^{N_{1}, N_{2}, N_{3}}(2, q)\right| \lesssim R N_{1}^{\theta+3 \kappa^{-0.1}}\left(N_{2} N_{3}\right)^{-\frac{\alpha}{2}+\epsilon_{1}} N_{2}^{1-\frac{\alpha}{2}}\left(N_{3}^{\frac{1}{2}}+N_{1}^{1-\frac{\alpha}{2}}\right)\left(L_{2} \wedge L_{3}\right)^{\frac{1}{2}}\left(L_{2} L_{3}\right)^{-\nu}
$$

(ii) If $N_{3} \ll N_{2} \ll N_{1}$ and $v_{3}$ is of type $(C)$, then

$$
\left|\Upsilon_{L_{2}, L_{3}}^{N_{1}, N_{2}, N_{3}}(q, 2)\right| \lesssim R N_{1}^{\theta+3 \kappa^{-0.1}}\left(N_{2} N_{3}\right)^{-\frac{\alpha}{2}+\epsilon_{1}} N_{3}^{1-\frac{\alpha}{2}}\left(N_{2}^{\frac{1}{2}}+N_{1}^{1-\frac{\alpha}{2}}\right)\left(L_{2} \wedge L_{3}\right)^{\frac{1}{2}}\left(L_{2} L_{3}\right)^{-\nu}
$$

(iii) If $N_{2} \ll N_{3} \ll N_{1}$ and $v_{2}$ is of type $(G)$, then

$$
\left|\Upsilon_{L_{2}, L_{3}}^{N_{1}, N_{2}, N_{3}}(q, q)\right| \lesssim R N_{1}^{\theta+3 \kappa^{-0.1}}\left(N_{2} N_{3}\right)^{-\frac{\alpha}{2}+\epsilon_{1}} N_{2}^{\frac{1}{2}}\left(N_{3}^{\frac{1}{2}}+N_{1}^{1-\frac{\alpha}{2}}\right) L_{3}^{-\nu}
$$

(iv) If $N_{3} \ll N_{2} \ll N_{1}$ and $v_{3}$ is of type $(G)$, then

$$
\left|\Upsilon_{L_{2}, L_{3}}^{N_{1}, N_{2}, N_{3}}(q, q)\right| \lesssim R N_{1}^{\theta+3 \kappa^{-0.1}}\left(N_{2} N_{3}\right)^{-\frac{\alpha}{2}+\epsilon_{1}} N_{3}^{\frac{1}{2}}\left(N_{2}^{\frac{1}{2}}+N_{1}^{1-\frac{\alpha}{2}}\right) L_{2}^{-\nu}
$$

(v) If $N_{2} \sim N_{3} \ll N_{1}$ and at least one of $v_{2}, v_{3}$ is of type (C), then

$$
\left|\Upsilon_{L_{2}, L_{3}}^{N_{1}, N_{2}, N_{3}}(2, q)\right| \lesssim R N_{1}^{\theta+3 \kappa^{-0.1}} N_{1}^{1-\frac{\alpha}{2}} N_{2}^{-\frac{7(\alpha-1)}{4}+2 \epsilon_{1}}\left(L_{2} L_{3}\right)^{-\nu}
$$

(vi) If $N_{2} \sim N_{3} \ll N_{1}$ and $v_{2}, v_{3}$ are both of type $(G)$, then

$$
\left|\Upsilon_{L_{2}, L_{3}}^{N_{1}, N_{2}, N_{3}}(q, q)\right| \lesssim R N_{1}^{\theta+3 \kappa^{-0.1}} N_{2}^{2 \epsilon_{1}}\left(N_{2}^{-(\alpha-1)}+N_{1}^{1-\frac{\alpha}{2}} N_{2}^{\frac{1}{2}-\alpha}\right)
$$

Proof. Since in the regime $N_{2}, N_{3} \ll N_{1}$, there is no significant difference between the second and the third place in the multi-linear expression, so without loss of generality, we may assume that $N_{2} \leq N_{3}$. By executing Algorithm A3, it suffices to estimate the input constant $\Lambda_{2,3}\left(N_{1}, N_{2}, N_{3}, L_{2}, L_{3}\right)$ in (7.28). Since in this step, we do not operate on modulation variables $\lambda, \lambda_{j}, \mu_{0}$, we will omit these variables below to simplify the notation. Recall that the multi-linear expression $G_{k, k_{1}}$ is given by (7.25), we have

$$
\begin{aligned}
\sum_{k_{1} \neq k} \mathbb{E}^{\mathcal{C}}\left[\left|G_{k_{1}, k}\right|^{2}\right] \leq & \sum_{\substack{k_{1}, k_{2}, k_{2}^{\prime}, k_{3}, k_{3}^{\prime} \\
k_{2}-k_{2}^{\prime}=k_{3}-k_{3}^{\prime}\left|k_{2}^{\prime}, k_{3}^{*}, k_{2}^{\prime *}, k_{3}^{\prime *}\\
\right| k_{3}^{\prime}-k_{3}^{\prime \prime} \mid<L_{3} N_{3}^{\epsilon}}} \bar{h}_{k_{2} k_{2}^{*}}^{(2)} h_{k_{2}^{\prime} k_{2}^{\prime *}}^{(2)} h_{k_{3} k_{3}^{*}}^{(q)} \bar{h}_{k_{3}^{\prime} k_{3}^{\prime *}}^{(q)} S_{k_{1}, k_{2}, k_{3}} S_{k_{1}, k_{2}^{\prime}, k_{3}^{\prime}} \\
& \times \mathbb{E}\left[\bar{g}_{k_{2}^{*}} g_{k_{2}^{\prime *}} g_{k_{3}^{*}} \bar{g}_{k_{3}^{\prime *}}\right] \cdot \frac{1}{\left[k_{2}^{*}\right]^{\frac{\alpha}{2}}\left[k_{2}^{\prime *}\right]^{\frac{\alpha}{2}}\left[k_{3}^{*}\right]^{\frac{\alpha}{2}}\left[k_{3}^{\prime *}\right]^{\frac{\alpha}{2}}} .
\end{aligned}
$$

\footnotetext{
${ }^{10}$ For fixed $v_{2}, v_{3}$ of characteristic parameters $\left(L_{2}, N_{2}\right),\left(L_{3}, N_{3}\right)$, we are free to choose $r_{2}, r_{3} \in\{2, q\}$, meaning that we specify in a priori the norms of $h^{N_{2}, L_{2}}$ and $h^{N_{3}, L_{3}}$ to be used. However, we are not able to use both norms of $h^{N_{2}, L_{2}}, h^{N_{3}, L_{3}}$ in a single multi-linear expression.
} 
Note that the only non-zero contributions in the summation are $k_{2}^{*}=k_{2}^{\prime *}, k_{3}^{*}=k_{3}^{\prime *}$ and $k_{2}^{*}=k_{3}^{*}, k_{2}^{* *}=k_{3}^{\prime *}$. Denote by $\mathcal{S}_{1}$ the contribution from $k_{2}^{*}=k_{2}^{\prime *}$ and $k_{3}^{*}=k_{3}^{\prime *}$ and by $\mathcal{S}_{2}$ the contribution from $k_{2}^{*}=k_{3}^{*}$ and $k_{2}^{\prime *}=k_{3}^{* *}$. Note that $\mathcal{S}_{2}=0$ if $N_{2} \ll N_{3}$ or $v_{2}, v_{3}$ are both of type $(\mathrm{G})$.

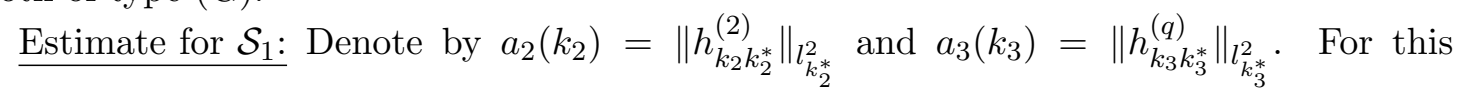
contribution we must have $\left|k_{2}-k_{2}^{\prime}\right| \lesssim L_{2} N_{3}^{\epsilon}$ and $\left|k_{3}-k_{3}^{\prime}\right| \lesssim L_{3} N_{3}^{\epsilon}$. Since $k_{2}-k_{2}^{\prime}=k_{3}-k_{3}^{\prime}$, we may change the constraint to $\left|k_{2}-k_{2}^{\prime}\right|=\left|k_{3}-k_{3}^{\prime}\right| \lesssim\left(L_{2} \wedge L_{3}\right) N_{3}^{\epsilon}$. By Cauchy-Schwartz, we have

$$
\begin{aligned}
\mathcal{S}_{1} & \lesssim\left(N_{2} N_{3}\right)^{-\alpha} \sum_{\substack{k_{1}, k_{2}, k_{2}^{\prime}, k_{3}, k_{3}^{\prime} \\
k_{2}-k_{2}^{\prime}=k_{3}-k_{3}^{\prime}}} \mathbf{1}_{\left|k_{2}-k_{2}^{\prime}\right| \lesssim\left(L_{2} \wedge L_{3}\right) N_{3}^{\epsilon}} S_{k_{1}, k_{2}, k_{3}} S_{k_{1}, k_{2}^{\prime}, k_{3}^{\prime}} a_{2}\left(k_{2}\right) a_{2}\left(k_{2}^{\prime}\right) a_{3}\left(k_{3}\right) a_{3}\left(k_{3}^{\prime}\right) \\
& \lesssim\left(N_{2} N_{3}\right)^{-\alpha}\left\|a_{3}\right\|_{l_{k_{3}}^{\infty}}^{2} \sum_{k_{2}, k_{2}^{\prime}, k_{3}} a_{2}\left(k_{2}\right) a_{2}\left(k_{2}^{\prime}\right) N_{1}^{\epsilon} \mathbf{1}_{\left|k_{2}-k_{2}^{\prime}\right| \lesssim\left(L_{2} \wedge L_{3}\right) N_{3}^{\epsilon}}\left(1+\frac{N_{1}^{2-\alpha}}{\left\langle k_{2}-k_{3}\right\rangle}\right),
\end{aligned}
$$

where to the last inequality, we use the counting bound $\sum_{k_{1}} S_{k_{1}, k_{2}, k_{3}} \lesssim N_{1}^{\epsilon}\left(1+\frac{N_{1}^{2-\alpha}}{\left\langle k_{2}-k_{3}\right\rangle}\right)$. Using Young's convolution inequality to the sum $\sum_{k_{2}, k_{2}^{\prime}} a_{2}\left(k_{2}\right) a_{2}\left(k_{2}^{\prime}\right) \mathbf{1}_{\left|k_{2}-k_{2}^{\prime}\right| \lesssim\left(L_{2} \wedge L_{3}\right) N_{3}^{\epsilon}}$, we have

$$
\mathcal{S}_{1} \lesssim\left(N_{2} N_{3}\right)^{-\alpha}\left(L_{2} \wedge L_{3}\right) N_{1}^{2 \epsilon}\left(N_{3}+N_{1}^{2-\alpha}\right)\left\|a_{2}\right\|_{l_{k_{2}}^{2}}^{2}\left\|a_{3}\right\|_{l_{k_{3}}^{\infty}}^{2}
$$

When $v_{2}$ is of type $(\mathrm{G})$, we could estimate $a_{2}\left(k_{2}\right)$ by $\left\|a_{2}\right\|_{l_{k_{2}}}$ and

$$
\mathcal{S}_{1} \lesssim\left(N_{2} N_{3}\right)^{-\alpha}\left\|a_{2}\right\|_{l^{\infty}}^{2}\left\|a_{3}\right\|_{l^{\infty}}^{2} N_{3}^{\epsilon}\left(N_{2} N_{3}+N_{1}^{2-\alpha} N_{2}\right) .
$$

Estimate for $\mathcal{S}_{2}$ : Note that $\mathcal{S}_{2} \neq 0$ only if $N_{2} \sim N_{3}$ and at least one of $v_{2}, v_{3}$ is of type (C). Therefore, without loss of generality, we may assume that $v_{2}$ is of type (C). Moreover, we have the constraint $\left|k_{2}-k_{3}\right|=\left|k_{2}^{\prime}-k_{3}^{\prime}\right| \lesssim\left(L_{2} \vee L_{3}\right) N_{3}^{\epsilon}$. By Cauchy-Schwartz,

$$
\begin{aligned}
\mathcal{S}_{2} & \lesssim\left(N_{2} N_{3}\right)^{-\alpha}\left\|a_{3}\right\|_{l_{k_{3}}^{\infty}}^{2} \sum_{\substack{k_{1}, k_{2}, k_{2}^{\prime}, k_{3}, k_{3}^{\prime} \\
k_{2}-k_{2}^{\prime}=k_{3}-k_{3}^{\prime}}} \mathbf{1}_{\left|k_{2}-k_{3}\right| \lesssim\left(L_{2} \vee L_{3}\right) N_{3}^{\epsilon}} S_{k_{1}, k_{2}, k_{3}} S_{k_{1}, k_{2}^{\prime}, k_{3}^{\prime}} a_{2}\left(k_{2}\right) a_{2}\left(k_{2}^{\prime}\right) \\
& \lesssim\left(N_{2} N_{3}\right)^{-\alpha}\left\|a_{3}\right\|_{l_{k_{3}}^{\infty}}^{2} \sum_{k_{1}, k_{2}, k_{3}} \mathbf{1}_{\left|k_{2}-k_{3}\right| \lesssim\left(L_{2} \vee L_{3}\right) N_{3}^{\epsilon}} S_{k_{1}, k_{2}, k_{3}} a_{2}\left(k_{2}\right) N_{1}^{\epsilon}\left\|a_{2}\right\|_{l_{k_{2}^{\prime}}^{\prime}}\left(1+\frac{N_{2}^{1-\frac{\alpha}{2}}}{\left\langle k_{2}-k_{3}\right\rangle^{\frac{1}{2}}}\right),
\end{aligned}
$$

where to the last inequality we use Cauchy-Schwartz for the sum in $k_{2}^{\prime}$ and the counting bound

$$
\sum_{k_{2}^{\prime}} S_{k_{1}, k_{2}^{\prime}, k_{3}+k_{2}^{\prime}-k_{2}} \lesssim N_{1}^{\epsilon}\left(1+\frac{N_{2}^{2-\alpha}}{\left\langle k_{2}-k_{3}\right\rangle}\right) .
$$

Using again $\sum_{k_{1}} S_{k_{1}, k_{2}, k_{3}} \lesssim N_{1}^{\epsilon}\left(1+\frac{N_{1}^{2-\alpha}}{\left\langle k_{2}-k_{3}\right\rangle}\right)$, we have

$$
\begin{aligned}
\mathcal{S}_{2} & \lesssim\left(N_{2} N_{3}\right)^{-\alpha} N_{1}^{2 \epsilon}\left\|a_{3}\right\|_{l_{k_{3}}^{\infty}}^{2}\left\|a_{2}\right\|_{l_{k_{2}}^{2}} \sum_{\substack{k_{2}, k_{3} \\
\left|k_{2}-k_{3}\right| \leq\left(L_{2} \vee L_{3}\right) N_{3}^{\epsilon}}} a_{2}\left(k_{2}\right)\left(1+\frac{N_{2}^{1-\frac{\alpha}{2}}}{\left\langle k_{2}-k_{3}\right\rangle^{\frac{1}{2}}}+\frac{N_{1}^{2-\alpha}}{\left\langle k_{2}-k_{3}\right\rangle}+\frac{N_{1}^{2-\alpha} N_{2}^{1-\frac{\alpha}{2}}}{\left\langle k_{2}-k_{3}\right\rangle^{\frac{3}{2}}}\right) \\
& \lesssim\left(N_{2} N_{3}\right)^{-\alpha} N_{1}^{3 \epsilon}\left(\left(L_{2} \vee L_{3}\right) N_{2}^{\frac{1}{2}}+\left(L_{2} \vee L_{3}\right)^{\frac{1}{2}} N_{2}^{\frac{3}{2}-\frac{\alpha}{2}}+N_{1}^{2-\alpha} N_{2}^{\frac{3}{2}-\frac{\alpha}{2}}\right)\left\|a_{3}\right\|_{l_{k_{3}}^{\infty}}^{2}\left\|a_{2}\right\|_{l_{k_{2}}^{2}}^{2} \\
& \leq\left(N_{2} N_{3}\right)^{-\alpha} N_{1}^{3 \epsilon} \cdot N_{1}^{2-\alpha} N_{2}^{\frac{3-\alpha}{2}}\left\|a_{3}\right\|_{l_{k_{3}}^{\infty}}^{2}\left\|a_{2}\right\|_{l_{k_{2}}^{2}}^{2} \\
& \lesssim N_{1}^{2-\alpha+3 \epsilon} N_{2}^{\frac{3-5 \alpha}{2}}\left\|a_{3}\right\|_{l_{k_{3}}^{\infty}}^{2}\left\|a_{2}\right\|_{l_{k_{2}}^{2}}^{2},
\end{aligned}
$$

since $\alpha<\frac{4}{3}$ and $N_{2} \sim N_{3}$. 
In summary, if at least one of $v_{2}, v_{3}$ is of type (C), when $N_{2} \ll N_{3}$, we obtain the upper bound

$$
\Lambda_{2,3}\left(N_{1}, N_{2}, N_{3}, L_{2}, L_{3}\right)=\left(N_{2} N_{3}\right)^{-\alpha}\left(L_{2} \wedge L_{3}\right) N_{1}^{2 \epsilon}\left(N_{3}+N_{1}^{2-\alpha}\right),
$$

and when $N_{2} \sim N_{3}$, we obtain the upper bound

$$
\Lambda_{2,3}\left(N_{1}, N_{2}, N_{3}, L_{2}, L_{3}\right)=N_{1}^{2-\alpha+3 \epsilon} N_{2}^{\frac{3-5 \alpha}{2}} .
$$

By choosing $4 \epsilon<\kappa^{-0.1}$, plugging into $(7.28)$ and using the corresponding $Z^{b_{0}}$ and $S^{b_{0}, q}$ bounds of $h^{N_{j} L_{j}}$ (note that $\frac{\alpha}{q_{0}}, \frac{1}{q}<\kappa^{-0.1}$ ), the proof of Lemma 7.5 is complete.

For $\Xi_{L_{2}, L_{3}}^{N_{1}, N_{2}, N_{3}}$, we have:

Lemma 7.6. We normalize $\left\|y_{k k_{1}}^{0}\left(\lambda, \lambda_{1}\right)\right\|_{l_{k}^{1} L_{\lambda}^{q^{\prime}} L_{\lambda_{1}}^{2} l_{k_{1}}^{2}}=1$. Assume that $v_{2}, v_{3}$ are both of type $(G)$ or $(C)$ with characterized parameters $\left(N_{2}, L_{2}\right),\left(N_{3}, L_{3}\right)$ satisfying $L_{2} \vee L_{3} \ll$ $\left(N_{2} \wedge N_{3}\right)$. Then outside a set of probability $<\mathrm{e}^{-N_{1}^{\theta} R}$ :

(i) If $N_{2} \wedge N_{3} \ll N_{2} \vee N_{3} \ll N_{1}$, then

$$
\left|\Xi_{L_{2}, L_{3}}^{N_{1}, N_{2}, N_{3}}(q, q)\right| \lesssim R N_{1}^{\theta+3 \kappa^{-0.1}}\left(N_{2} \wedge N_{3}\right)^{-\frac{\alpha-1}{2}}\left(N_{2} \vee N_{3}\right)^{-\frac{\alpha}{2}+2 \epsilon_{1}}\left(L_{2} \wedge L_{3}\right)^{\frac{1}{2}}\left(L_{2} L_{3}\right)^{-\nu}
$$

(ii) If $N_{2} \sim N_{3} \ll N_{1}$ and at least one of $v_{2}, v_{3}$ is of type (C), then

$$
\left|\Xi_{L_{2}, L_{3}}^{N_{1}, N_{2}, N_{3}}(2, q)\right| \lesssim R N_{1}^{\theta+3 \kappa^{-0.1}} N_{2}^{-\frac{7(\alpha-1)}{4}+2 \epsilon_{1}}\left(L_{2} L_{3}\right)^{-\nu}
$$

(iii) If $N_{2} \sim N_{3} \ll N_{1}$ and $v_{2}, v_{3}$ are both of type $(G)$, then

$$
\left|\Xi_{L_{2}, L_{3}}^{N_{1}, N_{2}, N_{3}}(q, q)\right| \lesssim R N_{1}^{\theta+3 \kappa^{-0.1}} N_{2}^{\frac{1}{2}-\alpha+2 \epsilon_{1}}
$$

Proof. Without loss of generality, we may assume that $N_{2} \leq N_{3}$. First we assume that $N_{2} \ll N_{3}$. From Algorithm A3, it suffices to estimate, for fixed $|k| \in N_{1}$, the expression

$$
\begin{aligned}
\sum_{k_{1}: k_{1} \neq k} \mathbb{E}^{\mathcal{C}}\left[\left|G_{k_{1}, k}\right|^{2}\right] & \sim\left(N_{2} N_{3}\right)^{-\alpha} \sum_{\substack{k_{2}, k_{2}^{\prime}, k_{3}, k_{3}^{\prime} \\
k_{2}-k_{2}^{\prime}=k_{3}-k_{3}^{\prime}}} S_{k+k_{2}-k_{3}, k_{2}, k_{3}} S_{k+k_{2}^{\prime}-k_{3}^{\prime}, k_{2}^{\prime}, k_{3}^{\prime}} \\
& \times \sum_{k_{2}^{*}, k_{3}^{*}, k_{2}^{\prime *}, k_{3}^{\prime *}} \prod_{j=2}^{3} \mathbf{1}_{\substack{\left|k_{j}-k_{j}^{*}\right|<L_{j} N_{3}^{\epsilon} \\
\left|k_{j}^{\prime}-k_{j}^{\prime *}\right|<L_{j} N_{3}^{\epsilon}}} \cdot \bar{h}_{k_{2} k_{2}^{*}}^{(q)} h_{k_{2}^{\prime} k_{2}^{\prime *}}^{(q)} h_{k_{3} k_{3}^{*}}^{(q)} \bar{h}_{k_{3}^{\prime} k_{3}^{\prime *}}^{(q)} \cdot \mathbb{E}\left[\bar{g}_{k_{2}^{*}} g_{k_{2}^{\prime *}} g_{k_{3}^{*}} \bar{g}_{k_{3}^{\prime *}}\right] .
\end{aligned}
$$

Since $N_{2} \ll N_{3}$ and $\left|k_{j}^{*}\right| \sim N_{j}$, the only non-zero contribution comes from $k_{2}^{*}=k_{2}^{\prime *}$ and $k_{3}^{*}=k_{3}^{\prime *}$. Consequently, since $k_{2}-k_{2}^{\prime}=k_{3}-k_{3}^{\prime},\left|k_{j}-k_{j}^{\prime}\right| \lesssim\left(L_{2} \wedge L_{3}\right) N_{3}^{\epsilon}, j=2,3$. Denote by $a_{j}\left(k_{j}\right)=\left\|h_{k_{j} k_{j}^{*}}^{(q)}\right\|_{l_{k_{j}^{*}}^{2}}$, by Cauchy-Schwartz, the quantity above can be bounded by

$$
\begin{aligned}
& \left(N_{2} N_{3}\right)^{-\alpha}\left\|a_{2}\right\|_{l_{k_{2}}}^{2}\left\|a_{3}\right\|_{l_{k_{3}}^{\infty}}^{2} \sum_{k_{2}, k_{2}^{\prime}} \mathbf{1}_{\left|k_{2}-k_{2}^{\prime}\right| \lesssim\left(L_{2} \wedge L_{3}\right) N_{3}^{\epsilon}} \sum_{k_{3}} S_{k+k_{2}-k_{3}, k_{2}, k_{3}} \\
\lesssim & \left(N_{2} N_{3}\right)^{-\alpha}\left\|a_{2}\right\|_{l_{k_{2}}^{\infty}}^{2}\left\|a_{3}\right\|_{l_{k_{3}}^{\infty}}^{2} \cdot N_{3}^{\epsilon}\left(L_{2} \wedge L_{3}\right) N_{2}=N_{2}^{-(\alpha-1)} N_{3}^{-\alpha+\epsilon}\left(L_{2} \wedge L_{3}\right)\left\|a_{2}\right\|_{l_{k_{2}}^{\infty}}^{2}\left\|a_{3}\right\|_{l_{k_{3}}^{\infty}}^{2} .
\end{aligned}
$$

Note that this estimate remains true if $N_{2} \sim N_{3}$ and $v_{2}, v_{3}$ are both of type (G). By choosing $\epsilon<\kappa^{-0.1}$ and noticing that $\frac{\alpha}{q_{0}}, \frac{1}{q}<\kappa^{-0.1}$, we have proved (i) and (iii).

Next we assume that $N_{2} \sim N_{3}$, then by Algorithm 3, to estimate $\Xi_{L_{2}, L_{3}}^{N_{1}, N_{2}, N_{3}}(2, q)$, it suffices to calculate 7.32 by changing $h_{k_{2} k_{2}^{*}}^{(q)}, h_{k_{2}^{\prime} k_{2}^{\prime *}}^{(q)}$ to $h_{k_{2} k_{2}^{*}}^{(2)}, h_{k_{2}^{\prime} k_{2}^{\prime *}}^{(2)}$. Denote by $\mathcal{S}_{1}$ the contribution from $k_{2}^{*}=k_{2}^{\prime *}, k_{3}^{*}=k_{3}^{\prime *}$ and by $\mathcal{S}_{2}$ the contribution from $k_{2}^{*}=k_{3}^{*}, k_{2}^{\prime *}=k_{3}^{\prime *}$. 
Estimate of $\mathcal{S}_{1}$ : by Cauchy-Schwartz, we have

$$
\begin{aligned}
\mathcal{S}_{1} & \lesssim\left(N_{2} N_{3}\right)^{-\alpha}\left\|a_{3}\right\|_{l_{k_{3}}^{\infty}}^{2} \sum_{k_{2}, k_{2}^{\prime}} a_{2}\left(k_{2}\right) a_{2}\left(k_{2}^{\prime}\right) \mathbf{1}_{\left|k_{2}-k_{2}^{\prime}\right|<\left(L_{2} \wedge L_{3}\right) N_{3}^{\epsilon}} \sum_{k_{3}} S_{k+k_{2}-k_{3}, k_{2}, k_{3}} \\
& \lesssim\left(N_{2} N_{3}\right)^{-\alpha}\left\|a_{3}\right\|_{l_{k_{3}}^{\infty}}^{2}\left\|a_{2}\right\|_{l_{k_{2}}^{2}}^{2}\left(L_{2} \wedge L_{3}\right) N_{3}^{\epsilon},
\end{aligned}
$$

where to the last inequality, we used Schur's test and the fact that $\sum_{k_{3}} S_{k+k_{2}-k_{3}, k_{2}, k_{3}} \lesssim 1$.

Estimate of $\mathcal{S}_{2}$ : by Cauchy-Schwartz and (7.31), we have

$$
\begin{aligned}
\mathcal{S}_{1} & \lesssim\left(N_{2} N_{3}\right)^{-\alpha}\left\|a_{3}\right\|_{l_{k_{3}}^{\infty}}^{2} \sum_{k_{2}, k_{2}^{\prime}, k_{3}} a_{2}\left(k_{2}\right) a_{2}\left(k_{2}^{\prime}\right) \mathbf{1}_{\left|k_{2}-k_{3}\right| \lesssim\left(L_{2} \vee L_{3}\right) N_{3}^{\epsilon}} S_{k+k_{2}-k_{3}, k_{2}, k_{3}} S_{k+k_{2}-k_{3}, k_{2}^{\prime}, k_{3}+k_{2}^{\prime}-k_{2}} \\
& \lesssim\left(N_{2} N_{3}\right)^{-\alpha}\left\|a_{3}\right\|_{l_{k_{3}}^{\infty}}^{2} \sum_{k_{2}, k_{3}} a_{2}\left(k_{2}\right) \mathbf{1}_{\left|k_{2}-k_{3}\right| \lesssim\left(L_{2} \vee L_{3}\right) N_{3}^{\epsilon}} S_{k+k_{2}-k_{3}, k_{2}, k_{3}}\left\|a_{2}\left(k_{2}^{\prime}\right)\right\|_{l_{k_{2}^{\prime}}^{2}} N_{1}^{\epsilon}\left(1+\frac{N_{2}^{1-\frac{\alpha}{2}}}{\left\langle k_{2}-k_{3}\right\rangle^{\frac{1}{2}}}\right) \\
& \lesssim\left(N_{2} N_{3}\right)^{-\alpha} N_{1}^{\epsilon}\left\|a_{3}\right\|_{l_{k_{3}}^{\infty}}^{2}\left\|a_{2}\right\|_{l_{k_{2}}^{2}} \sum_{k_{2}} a_{2}\left(k_{2}\right) \sum_{k_{3}} S_{k+k_{2}-k_{3}, k_{2}, k_{3}}\left(1+\frac{N_{2}^{1-\frac{\alpha}{2}}}{\left\langle k_{2}-k_{3}\right\rangle^{\frac{1}{2}}}\right) \\
& \lesssim N_{1}^{\epsilon}\left(N_{2} N_{3}\right)^{-\alpha}\left\|a_{3}\right\|_{l_{k_{3}}^{\infty}}^{2}\left\|a_{2}\right\|_{l_{k_{2}}^{2}}^{2} \cdot N_{2}^{\frac{1}{2}} \cdot N_{2}^{1-\frac{\alpha}{2}} \\
& \lesssim N_{1}^{\epsilon} N_{2}^{\frac{3-5 \alpha}{2}}\left\|a_{3}\right\|_{l_{k_{3}}^{\infty}}^{2}\left\|a_{2}\right\|_{l_{k_{2}}^{2}}^{2},
\end{aligned}
$$

since $\sum_{k_{3}} S_{k+k_{2}-k_{3}, k_{2}, k_{3}} \lesssim 1$. Implementing Algorithm 3, the proof of Lemma 7.6 is complete.

It remains to deal with the case where $L_{2} \vee L_{3} \gtrsim N_{2} \wedge N_{3}$, say $N_{2} \leq N_{3}$ and $L_{3} \gtrsim N_{2}$. Then from Lemma 7.1 and Lemma 7.3, we have

Lemma 7.7. We normalize $\left\|y_{k k_{1}}^{0}\left(\lambda, \lambda_{1}\right)\right\|_{L_{\lambda, \lambda_{1}}^{2} l_{k, k_{1}}^{2}}=1$. Assume that $v_{2}, v_{3}$ are both of type $(G)$ or $(C)$ with characterized parameters $\left(N_{2}, L_{2}\right),\left(N_{3}, L_{3}\right)$ satisfying $L_{2} \vee L_{3} \gtrsim N_{2} \wedge N_{3}$. Then

$$
\left|\Upsilon_{L_{2}, L_{3}}^{N_{1}, N_{2}, N_{3}}\right| \lesssim\left(N_{2} \wedge N_{3}\right)^{1-\frac{\alpha}{2}-\nu+\epsilon_{1}}\left(N_{2} \vee N_{3}\right)^{-\frac{\alpha}{2}+\frac{2}{q}+\epsilon_{2}}\left(\left(N_{2} \vee N_{3}\right)^{\frac{1}{2}}+N_{1}^{1-\frac{\alpha}{2}}\right)
$$

Proof. We apply (7.7) or (7.8), according to $N_{3} \leq N_{2}$ or $N_{2} \leq N_{3}$.

Lemma 7.8. We normalize $\left\|y_{k k_{1}}^{0}\left(\lambda, \lambda_{1}\right)\right\|_{l_{k}^{1} L_{\lambda}^{q^{\prime}} L_{\lambda_{1}}^{2} l_{k_{1}}^{2}}=1$. Assume that $v_{2}$, $v_{3}$ are both of type $(G)$ or $(C)$ with characterized parameters $\left(N_{2}, L_{2}\right),\left(N_{3}, L_{3}\right)$ satisfying $L_{2} \vee L_{3} \gtrsim$ $N_{2} \wedge N_{3}$. Then

$$
\left|\Xi_{L_{2}, L_{3}}^{N_{1}, N_{2}, N_{3}}\right| \lesssim\left(N_{2} \wedge N_{3}\right)^{1-\frac{\alpha}{2}-\nu+\epsilon_{1}}\left(N_{2} \vee N_{3}\right)^{-\frac{\alpha}{2}+\frac{1}{q}+\epsilon_{2}}
$$

Proof. We apply (7.11) or (7.12), according to $N_{3} \leq N_{2}$ or $N_{2} \leq N_{3}$.

- One random input Now we deal with the case where one of $v_{2}, v_{3}$ is of type $(\mathrm{G})$ or (C) and the other is of type (D).

Lemma 7.9. Assume that $\left\|y_{k k_{1}}^{0}\left(\lambda, \lambda_{1}\right)\right\|_{L_{\lambda, \lambda_{1}}^{2} l_{k, k_{1}}^{2}}=1$. Assume that one of $v_{2}, v_{3}$ is of type $(G)$ or $(C)$ and the other is of type (D). Then outside a set of probability $<\mathrm{e}^{-N_{1}^{\theta} R}$, the following estimates hold:

(i) If $N_{2} \ll N_{3} \ll N_{1}$ and $v_{3}$ is of type $(C)$ or $(G)$, we have

$$
\left|\Upsilon_{L_{2}, L_{3}}^{N_{1}, N_{2}, N_{3}}(2, q)\right| \lesssim R N_{1}^{\theta+3 \kappa^{-0.1}} N_{3}^{-\frac{\alpha}{2}+\epsilon_{1}} N_{2}^{\frac{1}{4}-s} L_{3}^{\frac{1}{4}-\nu}\left(N_{3}^{\frac{1}{2}}+N_{1}^{1-\frac{\alpha}{2}}\right)
$$

(ii) If $N_{3} \ll N_{2} \ll N_{1}$ and $v_{2}$ is of type $(G)$ or $(C)$, we have

$$
\left|\Upsilon_{L_{2}, L_{3}}^{N_{1}, N_{2}, N_{3}}(q, 2)\right| \lesssim R N_{1}^{\theta+3 \kappa^{-0.1}} N_{2}^{-\frac{\alpha}{2}+\epsilon_{1}} N_{3}^{\frac{1}{4}-s} L_{2}^{\frac{1}{4}-\nu}\left(N_{2}^{\frac{1}{2}}+N_{1}^{1-\frac{\alpha}{2}}\right)
$$


(iii) In any of these situations:

$-N_{2} \sim N_{3} \ll N_{1}$

$-N_{2} \ll N_{3} \ll N_{1}$ and $v_{3}$ is of type $(D)$;

$-N_{3} \ll N_{2} \ll N_{1}$ and $v_{2}$ is of type $(D)$; we have

$$
\left|\Upsilon_{L_{2}, L_{3}}^{N_{1}, N_{2}, N_{3}}(2,2)\right| \lesssim R N_{1}^{\theta+3 \kappa^{-0.1}}\left[N_{1}^{1-\frac{\alpha}{2}}+\left(N_{2} \wedge N_{3}\right)^{\frac{1}{2}}\right]\left(N_{2} \vee N_{3}\right)^{-s}\left(N_{2} \wedge N_{3}\right)^{-\frac{\alpha-1}{2}}
$$

Proof. Note that (iii) follows directly from Lemma 7.1, it suffices to prove (i) and (ii). The situations (i) and (ii) are similar, so we only prove (i). Recall that in this case, $N_{2} \ll N_{3}$, $v_{2}$ is of type (D) and $v_{3}$ is of type $(\mathrm{G})$ or $(\mathrm{C})$.

By executing Algorithm A2, we need to estimate (see $(7.17))^{11}$

$$
\underbrace{\left(\sum_{k_{2} \neq k_{2}^{\prime}} \mathbb{E}^{\mathcal{C}_{3}}\left[\left|\sigma_{k_{2} k_{2}^{\prime}}^{(3)}\right|^{2}\right]\right)^{\frac{1}{2}}}_{\text {I }}+\sup _{\left|k_{2}\right| \sim N_{2}} \underbrace{\left(\mathbb{E}^{\mathcal{C}_{3}}\left[\left|\sigma_{k_{2} k_{2}}^{(3)}\right|^{2}\right]\right)^{\frac{1}{2}}}_{\text {II }} .
$$

We calculate

$$
\begin{aligned}
& \mathrm{I}^{2}=\sum_{\substack{k_{2}, k_{2}^{\prime}, k_{1}, k_{3}, m_{1}, m_{3}, k_{3}^{\prime}, m_{3}^{\prime} \\
k_{2} \neq k_{2}^{\prime} \\
k_{3}-k_{3}^{\prime}=m_{3}-m_{3}^{\prime}=k_{2}-k_{2}^{\prime}}} S_{k_{1}, k_{2}, k_{3}} S_{k_{1}, k_{2}^{\prime}, k_{3}^{\prime}} S_{m_{1}, k_{2}, m_{3}} S_{m_{1}, k_{2}^{\prime}, m_{3}^{\prime}} \\
& \quad \times \sum_{\substack{\left|k_{3}^{*}\right| \sim N_{3},\left|k_{3}^{\prime *}\right| \sim N_{3} \\
\left|m_{3}^{*}\right| \sim N_{3},\left|m_{3}^{\prime *}\right| \sim N_{3}}} h_{k_{3} k_{3}^{*}}^{(q)} \bar{h}_{k_{3}^{\prime} k_{3}^{\prime *}}^{(q)} \bar{h}_{m_{3} m_{3}^{*}}^{(q)} h_{m_{3}^{\prime} m_{3}^{\prime *}}^{(q)} \cdot \frac{\mathbb{E}\left[g_{k_{3}^{*}} \bar{g}_{k_{3}^{\prime *}} \bar{g}_{m_{3}^{*}} g_{m_{3}^{\prime *}}\right]}{\left[k_{3}^{*}\right]^{\frac{\alpha}{2}}\left[k_{3}^{\prime *}\right]^{\frac{\alpha}{2}}\left[m_{3}^{*}\right]^{\frac{\alpha}{2}}\left[m_{3}^{\prime *}\right]^{\frac{\alpha}{2}}},
\end{aligned}
$$

where in the last line, the range of summation in $k_{3}^{*}, k_{3}^{\prime *}, m_{3}^{*}, m_{3}^{\prime *}$ satisfies:

$$
\left|k_{3}-k_{3}^{*}\right| \leq L_{3} N_{3}^{\epsilon},\left|k_{3}^{\prime}-k_{3}^{\prime *}\right| \leq L_{3} N_{3}^{\epsilon},\left|m_{3}-m_{3}^{*}\right| \leq L_{3} N_{3}^{\epsilon},\left|m_{3}^{\prime}-m_{3}^{\prime *}\right| \leq L_{3} N_{3}^{\epsilon}
$$

As usual, to simplify the notation, we omit the dependence on $\lambda_{3}, \mu_{0}$ and denote simply $h_{k_{3} k_{3}^{*}}^{(q)}=\left\langle\lambda_{3}\right\rangle^{\frac{2 b_{0}}{q^{\prime}}} \widetilde{h}_{k_{3} k_{3}^{*}}^{N_{3} L_{3}}\left(\lambda_{3}\right)$. Using the independence,

$$
\begin{aligned}
& \mathrm{I}^{2} \lesssim N_{3}^{-2 \alpha} \sum_{\substack{k_{2}, k_{2}^{\prime}, k_{1}, k_{3}, m_{1}, m_{3}, k_{3}^{\prime}, m_{3}^{\prime} \\
k_{2} \neq k_{1}^{\prime} \\
k_{2}-k_{2}^{\prime}=k_{3}-k_{3}^{\prime}=m_{3}-m_{3}^{\prime}}} S_{k_{1}, k_{2}, k_{3}} S_{k_{1}, k_{2}^{\prime}, k_{3}^{\prime}} S_{m_{1}, k_{2}, m_{3}} S_{m_{1}, k_{2}^{\prime}, m_{3}^{\prime}} \\
& \times \mathbb{E}[\underbrace{\mathbf{1}_{\left|k_{3}-k_{3}^{\prime}\right| \lesssim L_{3} N_{3}^{\epsilon}} \sum_{k_{3}^{*}, m_{3}^{*}}\left|h_{k_{3} k_{3}^{*}}^{(q)} \bar{h}_{k_{3}^{\prime} k_{3}^{*}}^{(q)} \bar{h}_{m_{3} m_{3}^{*}}^{(q)} h_{m_{3}^{\prime} m_{3}^{*}}^{(q)}\right|}_{\mathrm{I}_{1}}+\underbrace{\mathbf{1}_{\left|k_{3}-m_{3}\right| \lesssim L_{3} N_{3}^{\epsilon}} \sum_{k_{3}^{*}, k_{3}^{\prime *}} \mid h_{k_{3} k_{3}^{*}}^{(q)} \bar{h}_{m_{3} k_{3}^{*}}^{(q)} \bar{h}_{k_{3}^{\prime} k_{3}^{\prime *} h_{m_{3}^{\prime} k_{3}^{\prime *}}^{(q)} \mid}^{(q)} .}_{\mathrm{I}_{2}} .
\end{aligned}
$$

In the arguments below, we will not display the expectation $\mathbb{E}$ since we will not use the random feature of the coefficients $h_{k_{j} k_{j}^{*}}$.

Using Cauchy-Schwartz, we have

$$
\mathrm{I}_{1} \leq \mathbf{1}_{\left|k_{3}-k_{3}^{\prime}\right| \lesssim L_{3} N_{3}^{\epsilon}}\left\|\left.h_{k_{3} k_{3}^{*}}^{(q)}\right|_{l_{k_{3}}^{\infty} l_{k_{3}^{*}}^{2}} ^{4}, \quad \mathrm{I}_{2} \leq \mathbf{1}_{\left|k_{3}-m_{3}\right| \lesssim L_{3} N_{3}^{\epsilon}}\right\| h_{k_{3} k_{3}^{*}}^{(q)} \|_{l_{k_{3}}^{\infty} l_{k_{3}^{*}}^{2}}^{4}
$$

\footnotetext{
${ }^{11}$ To save the notation, in the summation below, we implicitly insert the constraint $\left|k_{2}\right|,\left|k_{2}^{\prime}\right| \leq$ $N_{2},\left|k_{3}\right|,\left|k_{3}^{\prime}\right|,\left|m_{3}\right|,\left|m_{3}^{\prime}\right| \sim N_{3}$.
} 
Plugging into 7.33 , the summation corresponding to the contribution $\mathrm{I}_{1}$ can be bounded by

$$
\begin{aligned}
& N_{3}^{-2 \alpha}\left\|h_{k_{3} k_{3}^{*}}^{(q)}\right\|_{l_{k_{3}} l_{k_{3}^{*}}^{2}}^{4} \sum_{\substack{k_{2}, k_{2}^{\prime} \\
0<\left|k_{2}-k_{2}^{\prime}\right| \lesssim L_{3} N_{3}^{\epsilon}}}\left(\sum_{k_{1}, k_{3}: k_{3} \neq k_{2}} S_{k_{1}, k_{2}, k_{3}} S_{k_{1}, k_{2}^{\prime}, k_{3}+k_{2}^{\prime}-k_{2}}\right)^{2} \\
& \lesssim N_{3}^{-2 \alpha}\left\|h_{k_{3} k_{3}^{*}}^{(q)}\right\|_{l_{k_{3}}^{\infty} l_{k_{3}^{*}}^{2}}^{4} \sum_{\substack{k_{2}, k_{2}^{\prime} \\
0<\left|k_{2}-k_{2}^{\prime}\right| \lesssim L_{3} N_{3}^{\epsilon}}}\left(N_{3}+N_{1}^{2-\alpha} N_{3}^{\epsilon}\right)^{2} \\
& \lesssim N_{3}^{-2 \alpha} N_{1}^{2 \epsilon} L_{3} N_{3}^{\epsilon} N_{2}\left[N_{3}^{2}+N_{1}^{2(2-\alpha)} N_{3}^{2 \epsilon}\right]\left\|h_{k_{3} k_{3}^{*}}^{(q)}\right\|_{l_{k_{3}}^{\infty} l_{k_{3}^{*}}^{2}}^{4}
\end{aligned}
$$

since

$$
\sum_{k_{3}: k_{3} \neq k_{2}} \sum_{k_{1}} S_{k_{1}, k_{2}, k_{3}} \lesssim \sum_{k_{3}} N_{1}^{\epsilon}\left(1+\frac{N_{1}^{2-\alpha}}{\left\langle k_{2}-k_{3}\right\rangle}\right) \lesssim N_{1}^{\epsilon}\left(N_{3}+N_{1}^{2-\alpha} N_{3}^{\epsilon}\right),
$$

thanks to the fact that $\left|k_{1}\right| \sim N_{1} \gg\left|k_{2}\right|,\left|k_{3}\right|$. Next we consider the summation corresponding to the contribution $\mathrm{I}_{2}$ which can be bounded by

$$
N_{3}^{-2 \alpha}\left\|h_{k_{3} k_{3}^{*}}^{(q)}\right\|_{l_{k_{3}}^{\infty} l_{k_{3}^{*}}^{2}}^{4} \sum_{\substack{k_{2}, k_{2}^{\prime}, k_{1}, m_{1}, k_{3}, m_{3} \\ k_{2} \neq k_{2}^{\prime} \\\left|k_{3}-m_{3}\right| \lesssim L_{3} N_{3}^{\epsilon}}} S_{k_{1}, k_{2}, k_{3}} S_{k_{1}, k_{2}^{\prime}, k_{3}+k_{2}^{\prime}-k_{2}} S_{m_{1}, k_{2}, m_{3}} S_{m_{1}, k_{2}^{\prime}, m_{3}+k_{2}^{\prime}-k_{2}}
$$

Since $\left|k_{2}\right|,\left|k_{2}^{\prime}\right| \leq N_{2} \ll N_{3} \sim\left|k_{3}\right|$ and $\left|\partial_{k_{2}^{\prime}} \Phi\left(k_{1}, k_{2}^{\prime}, k_{3}+k_{2}^{\prime}-k_{2}\right)\right| \sim N_{3}^{\alpha-1}>1$, we have, for fixed $k_{1}, k_{3}, k_{2}, \sum_{k_{2}^{\prime}} S_{k_{1}, k_{2}^{\prime}, k_{3}+k_{2}^{\prime}-k_{2}} \lesssim N_{1}^{\epsilon}$. Thus

$$
\begin{aligned}
& \sum_{\substack{k_{2}, k_{2}^{\prime}, k_{1}, m_{1}, k_{3}, m_{3} \\
k_{2} \neq k_{2}^{\prime}}} \mathbf{1}_{\left|k_{3}-m_{3}\right| \lesssim L_{3} N_{3}^{\epsilon}} S_{k_{1}, k_{2}, k_{3}} S_{k_{1}, k_{2}^{\prime}, k_{3}+k_{2}^{\prime}-k_{2}} S_{m_{1}, k_{2}, m_{3}} S_{m_{1}, k_{2}^{\prime}, m_{3}+k_{2}^{\prime}-k_{2}} \\
& \lesssim N_{1}^{\epsilon} \sum_{\substack{k_{2}, k_{3}, m_{3},\left|k_{3}-m_{3}\right| \lesssim L_{3} N_{3}^{\epsilon}}} \sum_{k_{1}, m_{1}} S_{k_{1}, k_{2}, k_{3}} S_{m_{1}, k_{2}, m_{3}} \\
& \lesssim \sum_{\substack{k_{2}, k_{3}, m_{3}: k_{2} \neq k_{3}, m_{3} \\
\left|k_{3}-m_{3}\right| \lesssim L_{3} N_{3}^{\epsilon}}} N^{3 \epsilon}\left(1+\frac{N_{1}^{2-\alpha}}{\left\langle k_{2}-k_{3}\right\rangle}\right)\left(1+\frac{N_{1}^{2-\alpha}}{\left\langle k_{2}-m_{3}\right\rangle}\right) \\
& \lesssim N_{1}^{2 \epsilon}\left[N_{1}^{2(2-\alpha)} N_{2}+L_{3} N_{2} N_{3}+L_{3} N_{2} N_{1}^{2-\alpha}\right],
\end{aligned}
$$

which is smaller than the upper bound (7.34) of $\mathrm{I}_{1}$. Therefore, we have

$$
\mathrm{I}^{\frac{1}{2}} \lesssim N_{3}^{-\frac{\alpha}{2}}\left\|h_{k_{3} k_{3}^{*}}^{(q)}\left(\lambda_{3}\right)\right\|_{l_{k_{3}}^{\infty} l_{k_{3}^{*}}^{2}} N_{1}^{2 \epsilon} N_{2}^{\frac{1}{4}} L_{3}^{\frac{1}{4}} \cdot\left(N_{3}^{\frac{1}{2}}+N_{1}^{1-\frac{\alpha}{2}}\right) .
$$

Next we estimate $\mathrm{II}^{2}$. For fixed $k_{2}$, we have

$$
\begin{aligned}
& \mathbb{E}^{\mathcal{C}_{3}}\left[\left|\sigma_{k_{2} k_{2}}^{(3)}\right|^{2}\right] \\
& =\mathbb{E}^{\mathcal{C}_{3}}\left[\left(\sum_{k_{1}, k: k_{1} \neq k}\left|w_{3}^{(q)}\left(k+k_{2}-k_{1}\right)\right|^{2} S_{k_{1}, k_{2}, k+k_{2}-k_{1}}\right)^{2}\right] \\
& =\mathbb{E}^{\mathcal{C}_{3}}\left[\sum_{\substack{k_{1}, k_{3}: k_{1}, k_{3} \neq k_{2} \\
m_{1}, m_{3}: m_{1}, m_{3} \neq k_{2}}} S_{k_{1}, k_{2}, k_{3}} S_{m_{1}, k_{2}, m_{3}} \mid \sum_{\substack{\left|k_{3}^{*}\right| \sim N_{3} \\
\left|k_{3}-k_{3}^{*}\right| \lesssim L_{3} N_{3}^{\epsilon}}} h_{k_{3} k_{3}^{*}}^{(q)}\left[\left.g_{k_{3}^{*}}\left[k_{3}^{*}\right]^{\frac{\alpha}{2}}\right|^{2}\left|\sum_{\substack{\left|m_{3}^{*}\right| \sim N_{3} \\
\left|m_{3}-m_{3}^{*}\right| \lesssim L_{3} N_{3}^{\epsilon}}} h_{m_{3} m_{3}^{*}}^{(q)}\left[g_{m_{3}^{*}} k_{3}^{*}\right]^{\frac{\alpha}{2}}\right|^{2}\right]\right. \\
& \sim N_{3}^{-2 \alpha}\left\|h_{k_{3} k_{3}^{*}}^{(q)}\right\|_{l_{k_{3}}^{\infty} l_{k_{3}^{*}}^{2}}^{4}\left(\sum_{k_{3}, k_{1}: k_{1}, k_{3} \neq k_{2}} S_{k_{1}, k_{2}, k_{3}}\right)^{2} \\
& \lesssim N_{3}^{-2 \alpha}\left\|h_{k_{3} k_{3}^{*}}^{(q)}\right\|_{l_{k_{3}}^{\infty} l_{3}^{2}}^{4}\left(\sum_{k_{3}: k_{3} \neq k_{2}} N_{1}^{\epsilon}\left(1+\frac{N_{1}^{2-\alpha}}{\left\langle k_{3}-k_{2}\right\rangle}\right)\right)^{2} \lesssim N_{3}^{-2 \alpha} N_{1}^{4 \epsilon}\left[N_{1}^{2(2-\alpha)}+N_{3}^{2}\right]\left\|h_{k_{3} k_{3}^{*}}^{(q)}\right\|_{l_{k_{3}}^{\infty} l_{k_{3}^{*}}^{2}}^{4} \text {. }
\end{aligned}
$$


Hence

$$
\mathrm{II}^{\frac{1}{2}} \lesssim\left[N_{1}^{1-\frac{\alpha}{2}+\epsilon}+N_{3}^{\frac{1}{2}} N_{1}^{\epsilon}\right] N_{3}^{-\frac{\alpha}{2}}\left\|h_{k_{3} k_{3}^{*}}^{(q)}\left(\lambda_{3}\right)\right\|_{l_{k_{3}}^{\infty} l_{k_{3}^{*}}^{2}},
$$

which is smaller than the upper bound $(7.34)$ of $\mathrm{I}^{\frac{1}{2}}$. Plugging into the output $(7.19)$ and choosing $\epsilon \ll \kappa^{-0.1}$, we complete the proof of Lemma 7.9 .

For the $\Xi_{L_{2}, L_{3}}^{N_{1}, N_{2}, N_{3}}$ norm, we have:

Lemma 7.10. Assume that $\left\|y_{k k_{1}}^{0}\left(\lambda, \lambda_{1}\right)\right\|_{l_{k}^{1} L_{\lambda}^{q^{\prime}} L_{\lambda_{1}}^{2} l_{k_{1}}^{2}}=1$. Assume that one of $v_{2}, v_{3}$ is of type $(G)$ or $(C)$ and the other is of type $(D)$. Then outside a set of probability $<\mathrm{e}^{-N_{1}^{\theta} R}$, the following estimates hold:

(i) If $N_{2} \ll N_{3} \ll N_{1}$ and $v_{3}$ is of type $(G)$ or $(C)$, we have

$$
\left|\Xi_{L_{2}, L_{3}}^{N_{1}, N_{2}, N_{3}}(2, q)\right| \lesssim R N_{1}^{\theta+3 \kappa^{-0.1}} N_{3}^{-\frac{\alpha}{2}} N_{2}^{\frac{1}{4}-s} \cdot N_{3}^{\epsilon_{1}} L_{3}^{-\nu}
$$

(ii) If $N_{3} \ll N_{2} \ll N_{1}$ and $v_{2}$ is of type $(G)$ or $(C)$, we have

$$
\left|\Xi_{L_{2}, L_{3}}^{N_{1}, N_{2}, N_{3}}(q, 2)\right| \lesssim R N_{1}^{\theta+3 \kappa^{-0.1}} N_{2}^{-\frac{\alpha}{2}} N_{3}^{\frac{1}{4}-s} \cdot N_{2}^{\epsilon 1} L_{2}^{-\nu}
$$

(iii) In any of these situations:

$-N_{2} \sim N_{3} \ll N_{1}$

$-N_{2} \ll N_{3} \ll N_{1}$ and $v_{3}$ is of type $(D)$;

then

$-N_{3} \ll N_{2} \ll N_{1}$ and $v_{2}$ is of type $(D)$;

$$
\left|\Xi_{L_{2}, L_{3}}^{N_{1}, N_{2}, N_{3}}(2,2)\right| \lesssim R N_{1}^{\theta+3 \kappa^{-0.1}}\left(N_{2} \vee N_{3}\right)^{-s}\left(N_{2} \wedge N_{3}\right)^{-\frac{\alpha-1}{2}}
$$

Proof. For similar reasons, it suffices to treat the situation (i) where $v_{2}$ is deterministic and $N_{3} \gg N_{2}$. Executing Algorithm A2, the key point is to control

$$
\sup _{k, k_{1}} \underbrace{\left(\sum_{k_{2}} \mathbb{E}^{\mathcal{C}_{3}}\left|\sigma_{k, k_{1} ; k_{2}}^{(3)}\right|^{2}\right)}_{\text {I }}, \quad \underbrace{\sup _{k, k^{\prime}}\left(\sum_{\substack{k_{1}, k_{1}^{\prime} \\\left(k, k_{1}\right) \neq\left(k^{\prime}, k_{1}^{\prime}\right)}} \mathbb{E}^{\left.\mathcal{C}_{3}\left|\sum_{k_{2}} \sigma_{k^{\prime}, k_{1}^{\prime} ; k_{2}}^{(3)} \bar{\sigma}_{k, k_{1} ; k_{2}}^{(3)}\right|^{2}\right)^{\frac{1}{2}},}\right.}_{\text {II }}
$$

where

$$
\sigma_{k, k_{1} ; k_{2}}^{(3)}=\sum_{\substack{k_{3}^{*}\left|k_{3}^{*}\right| \sim N_{3} \\\left|k_{3}-k_{3}^{*}\right| \leq L_{3} N_{3}^{\epsilon}}} h_{k+k_{2}-k_{1}, k_{3}^{*}}^{(q)} \frac{g_{k_{3}^{*}}}{\left[k_{3}^{*}\right]^{\frac{\alpha}{2}}} S_{k_{1}, k_{2}, k+k_{2}-k_{1}} .
$$

Using the independence and Cauchy-Schwartz

$$
\begin{aligned}
\mathrm{I}= & \sum_{\substack{k_{2}, k_{3}^{*}, k_{3}^{\prime}{ }^{*} \\
\left|k+k_{2}-k_{1}-k_{3}^{*}\right|<L_{3} N_{3}^{\epsilon}}} \mathbb{E}^{\mathcal{C}_{3}}\left[h_{k+k_{2}-k_{1}, k_{3}^{*}}^{(q)} \bar{h}_{k+k_{2}-k_{1}, k_{3}^{\prime *}}^{(q)} \frac{g_{k_{3}^{*}} \bar{g}_{k_{3}^{\prime *}}}{\left|k_{3}^{*}\right|^{\frac{\alpha}{2}}\left|k_{3}^{\prime *}\right|^{\frac{\alpha}{2}}} S_{k_{1}, k_{2}, k+k_{2}-k_{1}}\right] \\
& \quad \lesssim k_{2}-k_{1}-k_{3}^{\prime} \mid<L_{3} N_{3}^{\epsilon} \\
& \lesssim N_{1}^{\epsilon} N_{3}^{-\alpha}\left\|h_{k_{3} k_{3}^{*}}^{N_{3} L_{3}}\right\|_{l_{k_{3}} l_{k_{3}^{*}}}^{2},
\end{aligned}
$$

where we used $\sum_{k_{2}} S_{k_{1}, k_{2}, k+k_{2}-k_{1}} \lesssim N_{1}^{\epsilon}$. Next,

$$
\begin{aligned}
\mathrm{II}^{2}= & \sum_{\substack{k_{1}, k_{1}^{\prime}, k_{2}, m_{2} \\
\left(k, k_{1}\right) \neq\left(k_{1}, k_{1}^{\prime}\right)}} S_{k_{1}, k_{2}, k+k_{2}-k_{1}} S_{k_{1}^{\prime}, k_{2}, k^{\prime}+k_{2}-k_{1}^{\prime}} S_{k_{1}, m_{2}, k+m_{2}-k_{1}} S_{k_{1}^{\prime}, m_{2}, k^{\prime}+m_{2}-k_{1}^{\prime}} \\
& \times \sum_{k_{3}^{*}, k_{3}^{\prime *}, m_{3}^{*}, m_{3}^{\prime *}} h_{k+k_{2}-k_{1}, k_{3}^{*}} \bar{h}_{k^{\prime}+k_{2}-k_{1}^{\prime}, k_{3}^{\prime *}} \bar{h}_{k+m_{2}-k_{1}, m_{3}^{*}} h_{k^{\prime}+m_{2}-k_{1}^{\prime}, m_{3}^{*}} \frac{\mathbb{E}\left[g_{k_{3}^{*}} \bar{g}_{k_{3}^{\prime}} \bar{g}_{m_{3}^{*} g_{m_{3}^{*}}}\right]}{\left|k_{3}^{*}\right|^{\frac{\alpha}{2}}\left|k_{3}^{\prime *}\right|^{\frac{\alpha}{2}}\left|m_{3}^{*}\right|^{\frac{\alpha}{2}}\left|m_{3}^{\prime *}\right|^{\frac{\alpha}{2}}} .
\end{aligned}
$$


The non-zero contributions are $k_{3}^{*}=k_{3}^{\prime *}, m_{3}^{*}=m_{3}^{\prime *}$ and $k_{3}^{*}=m_{3}^{*}, k_{3}^{\prime *}=m_{3}^{\prime *}$.

For either the case $k_{3}^{*}=k_{3}^{\prime *}, m_{3}^{*}=m_{3}^{\prime *}$ or $k_{3}^{*}=m_{3}^{*}, k_{3}^{\prime *}=m_{3}^{\prime *}$, using Cauchy-Schwartz, we can estimate these contributions by

$$
\begin{aligned}
& N_{3}^{-2 \alpha}\left\|h_{k_{3} k_{3}^{*}}^{(q)}\right\|_{l_{k_{3}}^{\infty} l_{k_{3}^{*}}^{2}}^{4} \sum_{\substack{k_{1}, k_{1}^{\prime}, k_{2}, m_{2} \\
\left(k, k_{1}\right) \neq\left(k^{\prime}, k_{1}^{\prime}\right)}} S_{k_{1}, k_{2}, k+k_{2}-k_{1}} S_{k_{1}^{\prime}, k_{2}, k^{\prime}+k_{2}-k_{1}^{\prime}} S_{k_{1}, m_{2}, k+m_{2}-k_{1}} S_{k_{1}^{\prime}, m_{2}, k^{\prime}+m_{2}-k_{1}^{\prime}} \\
& \leq N_{3}^{-2 \alpha}\left\|h_{k_{3} k_{3}^{*}}^{(q)}\right\|_{l_{k_{3}}^{\infty} l_{k_{3}^{*}}^{2}}^{4} \sum_{k_{3}, k_{3}^{\prime}, k_{2}, m_{2}} S_{k+k_{2}-k_{3}, k_{2}, k_{3}} S_{k^{\prime}+k_{2}-k_{3}^{\prime}, k_{2}, k_{3}^{\prime}} S_{k+k_{2}-k_{3}, m_{2}, m_{2}+k_{3}-k_{2}} S_{k^{\prime}+k_{2}-k_{3}^{\prime}, m_{2}, m_{2}+k_{3}^{\prime}-k_{2} .} .
\end{aligned}
$$

Since $N_{1} \gg N_{3} \gg N_{2}$, we take the inner sum in the order $\sum_{k_{2}} \sum_{k_{3}} \sum_{k_{3}^{\prime}} \sum_{m_{2}}$. From

$$
\sum_{m_{2}} S_{k+k_{2}-k_{3}, m_{2}, m_{2}+k_{3}-k_{2}} \lesssim N_{1}^{\epsilon}
$$

and

$$
\sum_{k_{3}} S_{k+k_{2}-k_{3}, k_{2}, k_{3}} \sum_{k_{3}^{\prime}} S_{k^{\prime}+k_{2}-k_{3}^{\prime}, k_{2}, k_{3}^{\prime}} \lesssim 1
$$

finally we obtain that

$$
\mathrm{II}^{\frac{1}{4}} \lesssim N_{1}^{\epsilon} N_{3}^{-\frac{\alpha}{2}} N_{2}^{\frac{1}{4}}\left\|h_{k_{3} k_{3}^{*}}^{(q)}\right\|_{l_{k_{3}}^{\infty} l_{k_{3}^{*}}^{2}} .
$$

By implementing the output $(7.23)$ and choosing $\epsilon \ll \kappa^{-0.1}$, we complete the proof of Lemma 7.10 .

7.4. Kernel estimates. To finish the proof of Proposition 3.6 and Proposition 3.5, it suffices to estimate the right side of $(7.3)$ and $(7.4)$. Note that we ignore the error terms appearing in Proposition 6.1 here, as they are accompanied with some large negative power of $N_{1}$ which is negligible.

- Proof of Proposition 3.5: By symmetry, we may assume that $N_{2} \sim L$ is a fixed parameter and $N_{3} \leq N_{2}, L_{3}<N_{3}^{1-\delta}, L_{2}<N_{2}^{1-\delta}$ and $N_{1}>L^{\frac{1}{1-\delta}}$. First we note that, for fixed $\left(N_{1}, N_{2}, N_{3}, L_{1}, L_{2}, L_{3}\right)$, each time using Lemma 7.5. Lemma 7.6. Lemma 7.7. Lemma 7.8 and Lemma 7.9 , Lemma 7.10 , we should delete a set of probability $<\mathrm{e}^{-R N_{1}^{\theta}}$. Therefore, the probability of all the exceptional sets is bounded by

$$
\sum_{N_{3} \leq L, L_{1}, L_{2}, L_{3} \leq L} \sum_{N_{1}>L^{\frac{1}{1-\delta}}} O\left(\mathrm{e}^{-R N_{1}^{\theta}}\right)=O\left(\mathrm{e}^{-c R L^{\theta}}\right), \quad 0<c<1 .
$$

Therefore, outside a set of probability $<\mathrm{e}^{-c R L^{\theta}}$, we may assume that all the estimates in Lemma 7.5, Lemma 7.6, Lemma 7.7, Lemma 7.8 and Lemma 7.9, Lemma 7.10 hold. To finish the proof, we only need to estimate the sum over $N_{3} \leq N_{2}, L_{3}<N_{3}^{1-\delta}$ and $L_{2}<N_{2}^{1-\delta}$. To simplify the notation, we first pull out all possible small powers of $N_{1}, N_{2}, N_{3}$ like

$$
N_{1}^{\kappa^{-1}}, N_{1}^{\frac{\alpha}{q_{0}}+\frac{1}{q}}, N_{1}^{100\left(b-\frac{1}{2}\right)}, N_{2}^{\epsilon_{1}}, N_{2}^{\epsilon_{2}}, N_{3}^{\epsilon_{1}}, N_{3}^{\epsilon_{2}}
$$

in a unified on ${ }^{12} N_{1}^{100\left(\kappa^{-1}+b-\frac{1}{2}+\frac{1}{q}\right)} N_{2}^{10\left(\epsilon_{1}+\epsilon_{2}\right)}$ and ignore this small power in all the estimates below within this section. Recall the notation

$$
\Xi_{L_{2}, L_{3}}^{N_{1}, N_{2}, N_{3}}=\min \left\{\Xi_{L_{2}, L_{3}}^{N_{1}, N_{2}, N_{3}}\left(r_{2}, r_{3}\right):\left(r_{2}, r_{3}\right) \in\{2, q\} \times\{2, q\}\right\} .
$$

\footnotetext{
${ }^{12}$ The only caution is that the inductive small numbers $\epsilon_{1}, \epsilon_{2}$ should not fall on $N_{1}$.
} 
We split the sum into

$$
\begin{aligned}
& \sum_{\substack{N_{3}, L_{2}, L_{3} \\
N_{3}<N_{2}}} \Xi_{L_{2}, L_{3}}^{N_{1}, N_{2}, N_{3}}+\sum_{\substack{N_{3}, L_{2}, L_{3} \\
N_{3}<N_{2}}} \Xi_{L_{2}, L_{3}}^{N_{1}, N_{2}, N_{3}} \\
& \underbrace{L_{2}=2 L_{N_{2}}, L_{3}=2 L_{N_{3}}}_{\text {I }} \quad \underbrace{L_{2}<2 L_{N_{2}}, L_{3}<2 L_{N_{3}}}_{\text {II }} \\
& +\sum_{\substack{N_{3}, L_{2}, L_{3} \\
N_{3}<N_{2}}} \Xi_{L_{2}, L_{3}}^{N_{1}, N_{2}, N_{3}}+\sum_{\substack{N_{3}, L_{2}, L_{3} \\
N_{3}<N_{2}}} \Xi_{L_{2}, L_{3}}^{N_{1}, N_{2}, N_{3}} . \\
& \underbrace{L_{2}=2 L_{N_{2}}, L_{3}<2 L_{N_{3}}}_{\text {III }} \quad \underbrace{L_{2}<2 L_{N_{2}}, L_{3}=2 L_{N_{3}}}_{\text {IV }}
\end{aligned}
$$

Since all the inputs in I are of type (D), by Lemma 7.3 .

$$
\mathrm{I} \lesssim \sum_{N_{3}: N_{3} \leq N_{2}} N_{2}^{-s} N_{3}^{-s} \sim N_{2}^{-s}
$$

The inputs in II are all of type $(\mathrm{G})$ or $(\mathrm{C})$, by Lemma 7.6 and 7.8 , we have

$$
\begin{aligned}
\mathrm{II} & \lesssim \sum_{N_{3}: N_{3} \ll N_{2}} \sum_{L_{2}, L_{3} \ll N_{3}} N_{3}^{-\frac{\alpha-1}{2}} N_{2}^{-\frac{\alpha}{2}} L_{3}^{\frac{1}{2}-\nu} L_{2}^{-\nu}+\sum_{N_{3}: N_{3} \ll N_{2}} \sum_{\substack{L_{3} \ll N_{3} \\
N_{3} \lesssim L_{2} \ll N_{2}}} N_{3}^{1-\frac{\alpha}{2}-\nu} N_{2}^{-\frac{\alpha}{2}} \\
& +\sum_{N_{3}: N_{3} \sim N_{2}} \sum_{\substack{L_{2} \ll N_{2} \\
L_{3} \ll N_{3}}} N_{2}^{-\frac{7(\alpha-1)}{4}}\left(L_{2} L_{3}\right)^{-\nu}+N_{2}^{\frac{1}{2}-\alpha} \lesssim \log \left(N_{2}\right) N_{2}^{-(\alpha-1)-\nu}+N_{2}^{-\frac{7(\alpha-1)}{4}} .
\end{aligned}
$$

Next, applying Lemma 7.10 , we deduce that

$$
\mathrm{III}+\mathrm{IV} \lesssim N_{2}^{-s}+N_{2}^{-\frac{\alpha}{2}+\frac{1}{4}-s} \log N_{2} \lesssim N_{2}^{-s} .
$$

Therefore, since $\nu<\min \left\{s, \frac{7(\alpha-1)}{4}\right\}$ and $L=N_{2}$, we have proved Proposition 3.5.

- Proof of Proposition 3.6. As before, we ignore a unified factor $N_{1}^{100 \kappa^{-0.1}} N_{2}^{10\left(\epsilon_{1}+\epsilon_{2}\right)}$ and split the sum into I + II + III + IV as (7.36). From Lemma 7.1.

$$
\mathrm{I} \lesssim N_{1}^{1-\frac{\alpha}{2}} N_{2}^{-s}, \quad \text { if } N_{2}<N_{1}^{2-\alpha}
$$

and

$$
\text { I } \lesssim N_{1}^{1-\frac{\alpha}{2}} N_{2}^{-s}+N_{2}^{\frac{1}{2}-2 s}<N_{1}^{1-\frac{\alpha}{2}} N_{2}^{-s} \quad \text { if } N_{1}^{2-\alpha} \leq N_{2}\left(<N_{1}^{1-\delta}\right), \text { since } \alpha<\frac{4}{3} .
$$

To estimate II, we apply Lemma 7.5 and Lemma 7.7 and obtain that

$$
\begin{aligned}
\mathrm{II} & \lesssim \sum_{N_{3}: N_{3} \ll N_{2}} \sum_{\substack{L_{3}, L_{2} \ll N_{3} \\
L_{3}>\frac{1}{2}}}\left(N_{2} N_{3}\right)^{-\frac{\alpha}{2}} N_{3}^{1-\frac{\alpha}{2}}\left(N_{2}^{\frac{1}{2}}+N_{1}^{1-\frac{\alpha}{2}}\right) L_{2}^{\frac{1}{2}-\nu} L_{3}^{-\nu} \\
& +\sum_{N_{3}: N_{3} \ll N_{2}} \sum_{L_{2} \ll N_{3}}\left(N_{2} N_{3}\right)^{-\frac{\alpha}{2}} N_{3}^{\frac{1}{2}}\left(N_{2}^{\frac{1}{2}}+N_{1}^{1-\frac{\alpha}{2}}\right) L_{2}^{-\nu} \\
& +\sum_{N_{3}: N_{3} \ll N_{2}} \sum_{\substack{L_{2} \ll N_{2}, L_{3} \ll N_{3} \\
L_{2} \vee L_{3} \gtrsim N_{3}}} N_{3}^{1-\frac{\alpha}{2}-\nu} N_{2}^{-\frac{\alpha}{2}}\left(N_{2}^{\frac{1}{2}}+N_{1}^{1-\frac{\alpha}{2}}\right) \\
& +\sum_{N_{3}: N_{3} \sim N_{2}} \sum_{L_{2} \ll N_{2}, L_{3} \ll N_{3}} N_{1}^{1-\frac{\alpha}{2}} N_{2}^{-\frac{7(\alpha-1)}{4}}\left(L_{2} L_{3}\right)^{-\nu}+\left(N_{2}^{-(\alpha-1)}+N_{1}^{1-\frac{\alpha}{2}} N_{2}^{\frac{1}{2}-\alpha}\right) .
\end{aligned}
$$

Then direct computation gives

$$
\mathrm{II} \lesssim \log \left(N_{2}\right) N_{1}^{1-\frac{\alpha}{2}} N_{2}^{-\frac{\alpha-1}{2}-\nu}+N_{1}^{1-\frac{\alpha}{2}} N_{2}^{-\frac{7(\alpha-1)}{4}} .
$$


From Lemma 7.9 ,

$$
\mathrm{III} \lesssim \sum_{\substack{N_{3}, L_{2}, L_{3} \\ N_{3} \leq N_{2} \\ L_{2}=2 L_{N_{2}}, L_{3}<N_{3}^{1-\delta}}}\left(N_{1}^{1-\frac{\alpha}{2}}+N_{3}^{\frac{1}{2}}\right) N_{2}^{-s} N_{3}^{-\frac{\alpha-1}{2}} \lesssim N_{1}^{1-\frac{\alpha}{2}} N_{2}^{-s}+N_{2}^{1-\frac{\alpha}{2}-s} \log \left(N_{2}\right) \lesssim N_{1}^{1-\frac{\alpha}{2}} N_{2}^{-s},
$$

since $L \sim N_{2}<N_{1}^{1-\delta}$. Similarly,

$$
\begin{aligned}
\mathrm{IV} & \lesssim \sum_{N_{3}: N_{3} \ll N_{2}} \sum_{\substack{L_{2}, L_{3} \\
L_{2}<N_{2}^{1-\delta}, L_{3}=2 L_{N_{3}}}} N_{2}^{-\frac{\alpha}{2}} N_{3}^{\frac{1}{4}-s} L_{2}^{\frac{1}{4}-\nu}\left(N_{1}^{1-\frac{\alpha}{2}}+N_{2}^{\frac{1}{2}}\right) \\
& +\sum_{N_{3}: N_{3} \sim N_{2}} \sum_{\substack{L_{2}, L_{3} \\
L_{2}<N_{2}^{1-\delta}, L_{3}=2 L_{N_{3}}}}\left(N_{1}^{1-\frac{\alpha}{2}}+N_{3}^{\frac{1}{2}}\right) N_{2}^{-s} N_{3}^{-\frac{\alpha-1}{2}} \\
& \lesssim\left(N_{1}^{1-\frac{\alpha}{2}}+N_{2}^{\frac{1}{2}}\right) N_{2}^{-\frac{\alpha}{2}+\frac{1}{2}-\nu-s}+N_{2}^{1-\frac{\alpha}{2}-s} \log \left(N_{2}\right) \lesssim N_{1}^{1-\frac{\alpha}{2}} N_{2}^{-s} .
\end{aligned}
$$

Thus

$$
\mathrm{III}+\mathrm{IV} \lesssim N_{1}^{1-\frac{\alpha}{2}} N_{2}^{-s}
$$

Therefore, since $\nu \leq \min \left\{s, \frac{7(\alpha-1)}{4}\right\}-100\left(\epsilon_{1}+\epsilon_{2}\right)$ and $L=N_{2}$, we have proved Proposition 3.6 .

\section{REDUCTIONS AND ALGORITHMS FOR THE TRI-LINEAR ESTIMATES}

8.1. Reduction on the Fourier supports of type (D) and (C) terms. Before turning to concrete estimates, we will make some reductions, just in order to clean up the notations and the arguments below. We first reduce the estimate to the case where type (D) terms are localized in the Fourier space, in order to apply Proposition 6.2. This can be seen as follows: if some $v_{j}$ is of type (D), we will decompose it as

$$
\Pi_{N_{(1)}^{10}} v_{j}+\Pi_{N_{(1)}^{10}}^{\perp} v_{j} .
$$

Then by the bilinear Strichartz inequality, the contributions in $\mathcal{N}_{3}\left(v_{1}, v_{2}, v_{3}\right)$ when we replace $v_{j}$ by $\Pi_{N_{(1)}^{10}}^{\perp} v_{j}$ is negligible. Indeed, by duality, to estimate $\iint v_{1} \bar{v}_{2} v_{3} \bar{v} d x d t$, we split each $v_{i}$ into dyadic pieces and we have to estimate

$$
\sum_{M_{1}, M_{2}, M_{3}, M} \iint \mathbf{P}_{M_{1}} v_{1} \cdot \mathbf{P}_{M_{2}} \overline{v_{2}} \cdot \mathbf{P}_{M_{3}} v_{3} \cdot \mathbf{P}_{M} \bar{v} d x d t
$$

where at least one of $M_{1}, M_{2}, M_{3}$ is greater than $N_{(1)}^{10}$ and the corresponding function $v_{j}$ is of type (D). In particular, if for some $k, M_{k}=\max \left\{M_{1}, M_{2}, M_{3}\right\}$, then $M_{k}>N_{(1)}^{10}$ and $v_{k}$ must be of type (D), since each $v_{j}$ of type (G) or (C) has Fourier support $\left|k_{j}\right| \leq N_{j} \leq N_{(1)}$. By Hölder and the bilinear Strichartz inequality, we can bound the sum by

$$
\sum_{\substack{M_{1}, M_{2}, M_{3}, M \\ M_{(1) \geq N_{(1)}^{10}}}}\left(M_{(2)} M_{(3)}\right)^{\frac{1}{2}-\frac{\alpha}{4}}\left\|\mathbf{P}_{M_{1}} v_{1}\right\|_{X^{0, \frac{3}{8}}}\left\|\mathbf{P}_{M_{2}} v_{2}\right\|_{X^{0, \frac{3}{8}}}\left\|\mathbf{P}_{M_{3}} v_{3}\right\|_{X^{0, \frac{3}{8}}}\left\|\mathbf{P}_{M} v\right\|_{X^{0, \frac{3}{8}}} \cdot
$$

Note that if $v_{(j)}, j=2,3$ is not of type (D), then $M_{(j)} \leq N_{(1)}, j=2,3$, the dyadic summation converges and is bounded by $N_{(1)}^{-10 s}$, which is negligible. Therefore, from now on, we always assume that a term $v_{j}$ of type (D) has Fourier support $\left|k_{j}\right| \leq N_{(1)}^{10}$, hence the modulation reduction, Proposition 6.2 is always applicable.

Next, we claim that without loss of generality,

(H) for $v_{j}$ of type (D) or (C), we may further assume that

$$
\operatorname{supp}_{k_{j}}\left(\widehat{v}_{j}\right) \subset\left\{k_{j}:\left|k_{j}\right| \sim N_{j}\right\}
$$


and our goal is to prove an estimate of the form

$$
\left\|\mathcal{I N}\left(v_{1}, v_{2}, v_{3}\right)\right\|_{X^{0, b_{1}}} \lesssim R N_{(1)}^{-s-\delta_{0}}
$$

Indeed, if $v_{j}$ is of type (D), we can decompose it as

$$
v_{j}=\sum_{M_{j} \leq N_{(1)}^{10}} \mathbf{P}_{M_{j}} v_{j}
$$

By our assumption that $v_{j}$ has characterized frequency $N_{j}$, we have

$$
\left\|\mathbf{P}_{M_{j}} v_{j}\right\|_{X^{0, b}} \lesssim M_{j}^{-s}, \text { if } M_{j} \geq N_{j} ; \quad\left\|\mathbf{P}_{M_{j}} v_{j}\right\|_{X^{0, b}} \lesssim N_{j}^{-s}, \text { if } M_{j}<N_{j} .
$$

Then to estimate the $X^{0,1-b_{1}}$ norm of $\mathcal{N}\left(v_{1}, v_{2}, v_{3}\right)$, we can replace such $v_{j}$ by $\mathbf{P}_{M_{j}} v_{j}$ and then sum over every $M_{j} \leq N_{(1)}^{10}$. Note that the dyadic sum of $N_{j}^{-s}$ over $M_{j} \leq N_{j}$ only contributes $N_{j}^{-s} \log \left(N_{j}\right)$ and the dyadic sum of $M_{j}^{-s}$ over $M_{j}>N_{j}$ contributes $N_{j}^{-s}$, finally the logarithmic loss and the loss from the small powers $N_{(1)}^{100\left(\frac{1}{q}+b_{1}-0.5+\theta+\kappa^{-0.1}\right)}$ will be compensate by $N_{(1)}^{-\delta_{0}}$, thanks to Remark 3.1 . Similarly, if $v_{j}$ is of type (C), we decompose it as

$$
v_{j}=\sum_{\left|k_{j}\right| \sim N_{j}} \mathrm{e}_{k_{j}} \sum_{\substack{\left|k_{j}^{*}\right| \sim N_{j},\left|k_{j}-k_{j}^{*}\right| \leq L_{j} N_{j}^{\epsilon}}} h_{k_{j} k_{j}^{*}}^{N_{j} L_{j}}(t) \frac{g_{k_{j}^{*}}(\omega)}{\left[k_{j}^{*}\right]^{\frac{\alpha}{2}}}+\sum_{\left|k_{j}\right| \leq N_{j}} \mathrm{e}_{k_{j}} \sum_{\substack{\left|k_{j}^{*}\right| \sim N_{j},\left|k_{j}-k_{j}^{*}\right|>L_{j} N_{j}^{\epsilon}}} h_{k_{j} k_{j}^{*}}^{N_{j} L_{j}}(t) \frac{g_{k_{j}^{*}}(\omega)}{\left[k_{j}^{*}\right]^{\frac{\alpha}{2}}},
$$

where $\epsilon<\kappa^{-0.1}$. The second term is negligible in the estimate, compared with the first term on the right side. Indeed, we can treat the second term as a function of type $(D)^{13}$ with the $X^{0, b}$ bound $N_{j}^{10} \cdot N_{j}^{1-\kappa \epsilon} \ll N_{j}^{-10}$, thanks to (3.16). From these discussions, we always assume $(\mathbf{H})$ in the sequel and proceed to prove (8.1).

8.2. Reduction to the corresponding dyadic summations. Except for the highhigh-high interactions with at least one term of type (D), we can reduce the matter to several modes of summation, depending on how many random structures we want to exploit.

First, applying Proposition 6.2

$$
\begin{aligned}
\left\|\mathcal{I N}_{3}\left(v_{1}, v_{2}, v_{3}\right)\right\|_{X^{0, b_{1}}} & \lesssim N_{(1)}^{-100} \prod_{j=1}^{3}\left\|v_{j}\right\|_{X^{0, b}} \\
& +\left\|\mathcal{M}_{L_{1}, L_{2}, L_{3}}^{N_{1}, N_{2}, N_{3}}\left(\lambda_{1}, \lambda_{2}, \lambda_{3}, \mu_{0}, k\right)\right\|_{L_{\lambda_{1}}^{r_{1}} L_{\lambda_{2}}^{r_{2}} L_{\lambda_{3}}^{r_{3}} L_{\mu_{0}}^{q_{0}}\left(\left|\mu_{0}\right| \lesssim N_{(1)}^{\alpha}\right) l_{k}^{2}},
\end{aligned}
$$

where

$$
\mathcal{M}_{L_{1}, L_{2}, L_{3}}^{N_{1}, N_{2}, N_{3}}=\sum_{\left(k_{1}, k_{2}, k_{3}\right) \in \Gamma(k)} \widehat{\chi}\left(\mu_{0}-\Phi_{k_{1}, k_{2}, k_{3}}\right) \widetilde{w}_{1}\left(\lambda_{1}, k_{1}\right) \widetilde{\widetilde{w}}_{2}\left(\lambda_{2}, k_{2}\right) \widetilde{w}_{3}\left(\lambda_{3}, k_{3}\right),
$$

$q_{0}=\frac{1}{b_{1}-0.5}$, and $\widetilde{w}_{j}^{\left(r_{j}\right)}\left(\lambda_{j}, k_{j}\right)=\left\langle\lambda_{j}\right\rangle^{\frac{2 b_{0}}{r_{j}^{\prime}}} \widetilde{v}_{j}\left(\lambda_{j}, k_{j}\right)$ for $j=1,2,3$. We can ignore the error $N_{(1)}^{-100} \prod_{j=1}^{3}\left\|v_{j}\right\|_{X^{0, b_{0}}}$ and concentrate to the estimate of $\mathcal{M}_{L_{1}, L_{2}, L_{3}}^{N_{1}, N_{2}, N_{3}}$. As before, we may replace $\widehat{\chi}_{0}\left(\mu_{0}-\Phi_{k_{1}, k_{2}, k_{3}}\right)$ by $\mathbf{1}_{\Phi_{k_{1}, k_{2}, k_{3}}=\mu_{0}+O\left(N_{(1)}^{\epsilon}\right)}$. Moreover, we denote simply by $h_{k_{j} k_{j}^{*}}^{\left(r_{j}\right)}=$ $h_{k_{j} k_{j}^{*}}^{N_{j} L_{j},\left(r_{j}\right)}:=\left\langle\lambda_{j}\right\rangle^{\frac{2 b}{r_{j}^{\prime}}} \widetilde{h}_{k_{j} k_{j}^{*}}^{N_{j} L_{j}}\left(\lambda_{j}\right)$ when there is no risk of confusing. Recall also the notations:

$$
S_{k_{1}, k_{2}, k_{3}}:=\mathbf{1}_{k_{2} \neq k_{1}, k_{3}} \mathbf{1}_{\Phi_{k_{1}, k_{2}, k_{3}}=\mu_{0}+O\left(N_{(1)}^{\epsilon}\right)}, \quad\|\cdots\|_{L_{\mu_{0} *}^{q_{0}}}:=\|\cdots\|_{L_{\mu_{0}}^{q_{0}}\left(\left|\mu_{0}\right| \lesssim N_{(1)}^{\alpha}\right)}
$$

- Algorithm 1. Prototype: $v_{1}, v_{2}, v_{3}$ are all of type (G) or (C) Our algorithm in this case can be described as follows:

(a) Denote by $\mathcal{C}=\mathcal{B}_{\leq \max \left\{L_{1}, L_{2}, L_{3}\right\}}$, then $\mathcal{M}_{L_{1}, L_{2}, L_{3}}^{N_{1}, N_{2}, N_{3}}$ is a tri-linear expression of Gaussian

\footnotetext{
${ }^{13}$ We then perform the same Littlewood-Paley decomposition as we just did for the true type (D) terms.
} 
variables. When the coefficients are $\mathcal{C}$-measurable and are independent of all Gaussians in the expansion, we can apply Corollary 2.17 to deduce that outside a set of probability $<\mathrm{e}^{-N_{(1)}^{\theta} R^{\frac{2}{3}}}$

$$
\left\|\mathcal{M}_{L_{1}, L_{2}, L_{3}}^{N_{1}, N_{2}, N_{3}}\right\|_{L_{\lambda_{1}}^{q} L_{\lambda_{2}}^{q} L_{\lambda_{3}}^{q} L_{\mu_{0} *}^{q_{0}} l_{k}^{2}} \leq R N_{(1)}^{\theta}\left\|\left(\mathbb{E}^{\mathcal{C}}\left[\left|\mathcal{M}_{L_{1}, L_{2}, L_{3}}^{N_{1}, N_{2}, N_{3}}\right|^{2}\right]\right)^{\frac{1}{2}}\right\|_{L_{\lambda_{1}}^{q} L_{\lambda_{2}}^{q} L_{\lambda_{3}}^{q} L_{\mu_{0} *}^{q_{0}} l_{k}^{2}} .
$$

(b) As the crucial step, for fixed $\lambda_{1}, \lambda_{2}, \lambda_{3},\left|\mu_{0}\right| \lesssim N_{(1)}^{\alpha}$, we need to establish the following estimate:

$$
\mathbb{E}^{\mathcal{C}}\left[\left\|\mathcal{M}_{L_{1}, L_{2}, L_{3}}^{N_{1}, N_{2}, N_{3}}\right\|_{l_{k}^{2}}^{2}\right] \leq K\left(N_{1}, N_{2}, N_{3} ; L_{1}, L_{2}, L_{3}\right) \prod_{j=1}^{3}\left\|h_{k_{j} k_{j}^{*}}^{N_{j} L_{j},(q)}\left(\lambda_{j}\right)\right\|_{l_{k_{j}}^{\infty} l_{k_{j}^{*}}}^{2} .
$$

(c) Using the embedding $l^{\infty} \hookrightarrow l^{q}$, taking the square root of the output of the step (b) and then taking the $L_{\lambda_{1}}^{2} L_{\lambda_{2}}^{q} L_{\lambda_{3}}^{q} L_{\mu_{0} *}^{q_{0}}$ norm, we obtain that

$$
\left\|\mathcal{M}_{L_{1}, L_{2}, L_{3}}^{N_{1}, N_{2}, N_{3}}\right\|_{L_{\lambda_{1}}^{q} L_{\lambda_{2}}^{q} L_{\lambda_{3}}^{q} L_{\mu_{0} *}^{q_{0} L_{\omega}^{2}}} \leq N_{(1)}^{\frac{\alpha}{q_{0}}+\frac{3}{q}} K\left(N_{1}, N_{2}, N_{3}, L_{1}, L_{2}, L_{3}\right)^{\frac{1}{2}} \prod_{j=1}^{3}\left\|h^{N_{j} L_{j}}\right\|_{S^{b, q}} .
$$

From this algorithm, in practice, only the step (b) is not robust. By abusing the notations a bit, we can forget the modulation variable and ignore all the small powers of $N_{1}, N_{2}, N_{3}$ and write each $\widetilde{w}_{j}\left(\lambda_{j}, k_{j}\right)$ (with characterized parameters $\left(N_{j}, L_{j}\right)$ ) simply as $a_{j}\left(k_{j}\right)=a_{j}\left(k_{j}\right) \mathbf{1}_{\left|k_{j}\right| \sim N_{j}}$ and assume that:

- If $a_{j}$ is of type (C),

$$
\left\|h_{k_{j} k_{j}^{*}}^{N_{j} L_{j}}\right\|_{l_{k_{j}} l_{k_{j}^{*}}^{2}} \leq L_{j}^{-\nu}
$$

- If $a_{j}$ is of type (D),

$$
\left\|a_{j}\left(k_{j}\right)\right\|_{l_{k_{j}}^{2}} \lesssim N_{j}^{-s}
$$

- If $a_{j}$ is of type $(\mathrm{G})$,

$$
\left\|a_{j}\left(k_{j}\right)\right\|_{l_{k_{j}}^{2}} \lesssim N_{j}^{-\frac{\alpha-1}{2}} .
$$

Moreover, to make the notations cleaner, we will ignore all the small powers (in terms of $\left.\frac{1}{q}, \frac{1}{q_{0}} \sim b_{1}-0.5, \epsilon<\kappa^{-0.1}, \epsilon_{1}, \epsilon_{2}, \delta_{0}, \cdots\right)$ of $N_{j}$ and finally we multiply by a unified factor

$$
N_{(1)}^{\frac{\alpha}{q_{0}}+\frac{3}{q}+\theta+100 \kappa^{-0.1}+3 \epsilon_{1}+3 \epsilon_{2}}
$$

to the output.

In summary, by Algorithm 1, we need to establish an estimate

$$
\mathbb{E}\left[\left|\mathcal{U}_{L_{1}, L_{2}, L_{3}}^{N_{1}, N_{3}, N_{3}}\right|^{2}\right] \leq K_{1}\left(N_{1}, N_{2}, N_{3}\right),
$$

where

$$
\mathcal{U}_{L_{1}, L_{2}, L_{3}}^{N_{1}, N_{2}, N_{3}}=\left(\sum_{k}\left|\sum_{\left(k_{1}, k_{2}, k_{3}\right) \in \Gamma(k)} a_{1}\left(k_{1}\right) \bar{a}_{2}\left(k_{2}\right) a_{3}\left(k_{3}\right)\right|^{2}\right)^{\frac{1}{2}},
$$

with $a_{1}, a_{2}, a_{3}$ having characterized parameters $\left(N_{1}, L_{1}\right),\left(N_{2}, L_{2}\right),\left(N_{3}, L_{3}\right)$, with respectively, satisfying corresponding estimates $(8.2),(8.3)$ and (8.4). Then the output is

$$
\text { Output of Algorithm } 1 \leq R N_{(1)}^{\frac{\alpha}{q_{0}}+\frac{3}{q}+\theta+100\left(\kappa^{-0.1}+\epsilon_{1}+\epsilon_{2}\right)} K_{1}\left(N_{1}, N_{2}, N_{3}\right)^{\frac{1}{2}}
$$

- Algorithm 2. Prototype: at least two of $v_{1}, v_{2}, v_{3}$ are of type $(\mathbf{G})$ or $(\mathbf{C})$ 
We may assume that $v_{1}, v_{2}$ are of type $(\mathrm{G})$ or $(\mathrm{C})$ and we denote by $\mathcal{C}=\mathcal{B}_{\leq\left(L_{1} \vee L_{2}\right)}$. The algorithm can be described as follows:

(a)

$$
\left\|\mathcal{M}_{L_{1}, L_{2}, L_{3}}^{N_{1}, N_{2}, N_{3}}\right\|_{L_{\lambda_{1}}^{q} L_{\lambda_{2}}^{q} L_{\lambda_{3}}^{2} L_{\mu_{0} *}^{q_{0}} l_{k}^{2}} \leq\left\|\mathcal{G}_{3} \mathcal{G}_{3}^{*}\right\|_{L_{\lambda_{1}, \lambda_{2}}^{\frac{q}{2}} L_{\mu_{0} *}^{\frac{q_{0}}{2}} \mathcal{L}\left(l_{k_{3}}^{2}\right)}^{\frac{q^{2}}{2}}\left\|\widetilde{w}_{3}\left(\lambda_{3}, k_{3}\right)\right\|_{l_{k_{3}}^{2}},
$$

where the kernel of the random operator $\mathcal{G}_{3} \mathcal{G}_{3}^{*}$ (depending on $\lambda_{1}, \lambda_{2}$ and $\mu_{0}$ ) is given by

$$
\begin{gathered}
\sigma_{k, k^{\prime}}^{(3)}=\sum_{k_{2}, k_{2}^{\prime}, k_{3}} \overline{\widetilde{w}}_{1}\left(\lambda_{1}, k^{\prime}+k_{2}^{\prime}-k_{3}\right) \widetilde{w}_{1}\left(\lambda_{1}, k+k_{2}-k_{3}\right) \widetilde{w}_{2}\left(\lambda_{2}, k_{2}^{\prime}\right) \overline{\widetilde{w}}_{2}\left(\lambda_{2}, k_{2}\right) \\
\times S_{k^{\prime}+k_{2}^{\prime}-k_{3}, k_{2}^{\prime}, k_{3}} S_{k+k_{2}-k_{3}, k_{2}, k_{3} .}
\end{gathered}
$$

Then by Lemma 2.12 ,

$$
\left\|\mathcal{G}_{3} \mathcal{G}_{3}^{*}\right\|_{l_{k^{\prime}}^{2} \rightarrow l_{k}^{2}} \leq L \sup _{k, k^{\prime}:\left|k-k^{\prime}\right|<L}\left|\sigma_{k, k^{\prime}}^{(3)}\right|+\left(\sum_{k, k^{\prime}:\left|k-k^{\prime}\right| \geq L}\left|\sigma_{k, k^{\prime}}^{(3)}\right|^{2}\right)^{\frac{1}{2}} .
$$

(b) Since $\left|\sigma_{k, k^{\prime}}^{(3)}\right|^{2}$ is a bilinear expression of Gaussian variables, when the coefficients are independent of these Gaussians in the expansion, by Corollary 2.17, outside a set of probability $<\mathrm{e}^{-N_{1}^{\theta} R}$, we have

$$
\left\|\left(\sum_{k, k^{\prime}:\left|k-k^{\prime}\right| \geq L}\left|\sigma_{k, k^{\prime}}^{(3)}\right|^{2}\right)^{\frac{1}{2}}\right\|_{L_{\lambda_{1}, \lambda_{2}}^{\frac{q}{2}}} L_{\mu_{0^{*}}}^{\frac{q_{0}}{2}} \leq R N_{1}^{\theta}\left\|\left(\sum_{k, k^{\prime}:\left|k-k^{\prime}\right| \geq L} \mathbb{E}^{\mathcal{C}}\left[\left|\sigma_{k, k^{\prime}}^{(3)}\right|^{2}\right]\right)^{\frac{1}{2}}\right\|_{L_{\lambda_{1}, \lambda_{2}}^{\frac{q}{2}}} \frac{{\frac{q}{\mu_{0}}}^{\frac{q_{0}}{2}}}{2},
$$

and

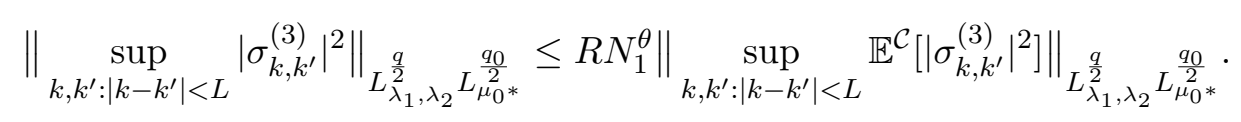

The main step is to establish the estimates

$$
\sum_{k, k^{\prime}:\left|k-k^{\prime}\right| \geq L} \mathbb{E}^{\mathcal{C}}\left[\left|\sigma_{k, k^{\prime}}^{(3)}\right|^{2}\right] \leq K\left(N_{1}, N_{2}, N_{3}, L_{1}, L_{2}, L_{3}\right) \prod_{j=1}^{2}\left\|h_{k_{j} k_{j}^{*}}^{N_{j} L_{j},(q)}\right\|_{l_{k_{j}}^{\infty} l_{k_{2}^{*}}^{2}}^{2}
$$

and

$$
\sup _{k, k^{\prime}:\left|k-k^{\prime}\right|<L} \mathbb{E}^{\mathcal{C}}\left[\left|\sigma_{k, k^{\prime}}^{(3)}\right|^{2}\right] \leq K^{\prime}\left(N_{1}, N_{2}, N_{3}, L_{1}, L_{2}, L_{3}\right) \prod_{j=1}^{2}\left\|h_{k_{j} k_{j}^{*}}^{N_{j} L_{j},(q)}\right\|_{l_{k_{j}}^{\infty} l_{k_{2}^{*}}^{2}}^{2} .
$$

(c) Having the bounds in the step (b), we deduce that outside a set of probability $<\mathrm{e}^{-N_{1}^{\theta} R}$,

$$
\begin{aligned}
& \left\|\mathcal{G}_{3} \mathcal{G}_{3}^{*}\right\|^{\frac{1}{2}}{ }_{L_{\lambda_{1}, \lambda_{2}}^{\frac{q}{2}}}^{\frac{q_{0}}{2}} L_{\mu_{0} *}^{\frac{q_{0}}{2}} \mathcal{L}\left(l_{k_{3}}^{2}\right) \\
\leq & R N_{1}^{\theta+\frac{\alpha}{q_{0}}+\frac{2}{q}}\left(L^{\frac{1}{2}} K^{\prime}\left(N_{1}, N_{2}, N_{3}, L_{1}, L_{2}, L_{3}\right)^{\frac{1}{4}}+K\left(N_{1}, N_{2}, N_{3}, L_{1}, L_{2}, L_{3}\right)^{\frac{1}{4}}\right) \prod_{j=1}^{2}\left\|h^{N_{j} L_{j}}\right\|_{S^{b}}^{\frac{1}{2}} .
\end{aligned}
$$

Again, since only the estimates for the expectations in the step (b) are not robust, by abusing the notations a bit, we may write each $\widetilde{w}_{j}\left(\lambda_{j}, k_{j}\right)$ (with characterized parameters $\left.\left(N_{j}, L_{j}\right)\right)$ simply as $a_{j}\left(k_{j}\right)=a_{j}\left(k_{j}\right) \mathbf{1}_{\left|k_{j}\right| \sim N_{j}}$ and making the same assumptions 8.2, 8.3 and (8.4) for type (C), (D) and (G) terms as in Algorithm 1, with respectively. Again, to make the notations cleaner, we will ignore all the small powers (in terms of $\left.\epsilon<\kappa^{-0.1}, \epsilon_{1}, \epsilon_{2}, \delta_{0}, \frac{1}{q}, \frac{1}{q_{0}} \sim b_{1}-0.5\right)$ of $N_{j}$ and finally we multiply by a unified factor $N_{(1)}^{\frac{\alpha}{q_{0}}+\frac{2}{q}+\theta+100 \kappa^{-0.1}+3 \epsilon_{1}+3 \epsilon_{2}}$ to the output.

Similarly, if $v_{1}, v_{3}$ are of type $(\mathrm{G})$ or $(\mathrm{C})$, we denote by $\mathcal{C}=\mathcal{B}_{\leq\left(L_{1} \vee L_{3}\right)}$ we will apply the above algorithm to the operator $\mathcal{G}_{2} \mathcal{G}_{2}^{*}$ with matrix elements

$$
\begin{gathered}
\sigma_{k, k^{\prime}}^{(2)}=\sum_{k_{3}, k_{3}^{\prime}, k_{2}} \overline{\widetilde{w}}_{1}\left(\lambda_{1}, k^{\prime}+k_{2}-k_{3}^{\prime}\right) \widetilde{w}_{1}\left(\lambda_{1}, k+k_{2}-k_{3}\right) \widetilde{w}_{3}\left(\lambda_{3}, k_{3}^{\prime}\right) \overline{\widetilde{w}}_{3}\left(\lambda_{3}, k_{3}\right) \\
\times S_{k^{\prime}+k_{2}-k_{3}^{\prime}, k_{2}, k_{3}^{\prime}} S_{k+k_{2}-k_{3}, k_{2}, k_{3} .}
\end{gathered}
$$


In summary, by Algorithm 2, we need to establish the bound:

$$
\sum_{j=2}^{3} \mathbb{E}^{\mathcal{C}}\left[\sum_{k, k^{\prime}:\left|k-k^{\prime}\right| \geq L}\left|\eta_{k, k^{\prime}}^{(j)}\right|^{2}\right]+\sup _{k, k^{\prime}:\left|k-k^{\prime}\right|<L} \mathbb{E}^{\mathcal{C}}\left[\left|\eta_{k, k^{\prime}}^{(j)}\right|^{2}\right] \leq K_{2}\left(N_{1}, N_{2}, N_{3}\right),
$$

where

(8.8)

$$
\eta_{k, k^{\prime}}^{(3)}=\sum_{k_{2}, k_{2}^{\prime}, k_{3}} \bar{a}_{1}\left(k^{\prime}+k_{2}^{\prime}-k_{3}\right) a_{1}\left(k+k_{2}-k_{3}\right) a_{2}\left(k_{2}^{\prime}\right) \bar{a}_{2}\left(k_{2}\right) S_{k^{\prime}+k_{2}^{\prime}-k_{3}, k_{2}^{\prime}, k_{3}} S_{k+k_{2}-k_{3}, k_{2}, k_{3}},
$$

$$
\eta_{k, k^{\prime}}^{(2)}=\sum_{k_{3}, k_{2}^{\prime}, k_{2}} \bar{a}_{1}\left(k^{\prime}+k_{2}-k_{3}^{\prime}\right) a_{1}\left(k+k_{2}-k_{3}\right) a_{3}\left(k_{3}^{\prime}\right) \bar{a}_{3}\left(k_{3}\right) S_{k^{\prime}+k_{2}-k_{3}^{\prime}, k_{2}, k_{3}^{\prime}} S_{k+k_{2}-k_{3}, k_{2}, k_{3}} .
$$

Then

$$
\text { Output of Algorithm } 2 \leq R N_{1}^{\frac{\alpha}{q_{0}}+\frac{2}{q}+\theta+100\left(\kappa^{-0.1}+\epsilon_{1}+\epsilon_{2}\right)} K_{2}\left(N_{1}, N_{2}, N_{3}\right)^{\frac{1}{4}}\left\|a_{2}\right\|_{l^{2}}
$$

or

$$
\text { Output of Algorithm } 2 \leq R N_{1}^{\frac{\alpha}{q_{0}}+\frac{2}{q}+\theta+100\left(\kappa^{-0.1}+\epsilon_{1}+\epsilon_{2}\right)} K_{2}\left(N_{1}, N_{2}, N_{3}\right)^{\frac{1}{4}}\left\|a_{3}\right\|_{l^{2}} .
$$

-Algorithm 3. Prototype: at least one term of type (G) or (C) Without loss of generality, we assume that $v_{1}$ is of type $(\mathrm{G})$ or $(\mathrm{C})$. By Cauchy-Schwartz, we have

$$
\begin{aligned}
& \left\|\mathcal{M}_{L_{1}, L_{2}, L_{3}}^{N_{1}, N_{2}, N_{3}}\right\|_{L_{\lambda_{1}}^{q} L_{\lambda_{2}, \lambda_{3}}^{2} L_{\mu_{0} *}^{q_{0}} l_{k}^{2}} \\
\leq & \left\|\widetilde{w}_{1}\left(\lambda_{1}, k_{1}\right)\right\|_{L_{\lambda_{1}}^{q} l_{k_{1}}}\left\|\left(\sum_{|k| \leq N_{(1)}}\left|\sum_{\left(k_{1}, k_{2}, k_{3}\right) \in \Gamma(k)} \widetilde{\widetilde{w}}_{2}\left(\lambda_{2}, k_{2}\right) \widetilde{w}_{3}\left(\lambda_{3}, k_{3}\right)\right|^{2}\right)^{\frac{1}{2}}\right\|_{L_{\lambda_{2}, \lambda_{3}}^{2} L_{\mu_{0} *}^{q_{0}}} .
\end{aligned}
$$

From the embedding $l^{\infty} \hookrightarrow l^{q}$, Hölder's inequality and Lemma 4.2, we deduce that, outside a set of probability $<\mathrm{e}^{-c N^{\theta} R^{2}}$,

$$
\left\|\widetilde{w}_{1}\left(\lambda_{1}, k_{1}\right)\right\|_{L_{\lambda_{1}}^{2} l_{k_{1}}^{\infty}} \leq N_{1}^{\frac{1}{q}}\left\|\widetilde{w}_{1}\left(\lambda_{1}, k_{1}\right)\right\|_{l_{k_{1}}^{\infty} L_{\lambda_{1}}^{q}} \lesssim N_{1}^{-\frac{\alpha}{2}+\epsilon_{1}+\frac{1}{q}} L^{-\nu} .
$$

Again, since the key step is to estimate the discrete sum, and the $L_{\lambda_{2}, \lambda_{3}}^{2} L_{\mu_{0} *}^{q_{0}}$ will only contribute a $N_{0}^{\frac{\alpha}{q_{0}}}$ factor, we may write each $\widetilde{w}_{j}\left(\lambda_{j}, k_{j}\right)$ (with characterized parameters $\left.\left(N_{j}, L_{j}\right)\right)$ simply as $a_{j}\left(k_{j}\right)=a_{j}\left(k_{j}\right) \mathbf{1}_{\left|k_{j}\right| \sim N_{j}}$ and assume that:

- If $a_{j}$ is of type (C),

$$
\left\|a_{j}\left(k_{j}\right)\right\|_{l_{k_{j}}} \leq N_{j}^{-\frac{\alpha}{2}} L_{j}^{-\nu}, \text { and }\left\|h_{k_{j} k_{j}^{*}}^{N_{j} L_{j}}\right\|_{l_{k_{j} k_{j}^{*}}^{\infty} l^{2}} \leq L_{j}^{-\nu}
$$

- If $a_{j}$ is of type (D),

$$
\left\|a_{j}\left(k_{j}\right)\right\|_{l_{k_{j}}^{2}} \lesssim N_{j}^{-s}
$$

- If $a_{j}$ is of type $(\mathrm{G})$,

$$
\left\|a_{j}\left(k_{j}\right)\right\|_{l_{k_{j}}^{2}} \lesssim N_{j}^{-\frac{\alpha-1}{2}},\left\|a_{j}\left(k_{j}\right)\right\|_{l_{k_{j}}^{\infty}} \leq N_{j}^{-\frac{\alpha}{2}}
$$

Similarly, to make the notations cleaner, we will ignore all the small powers (in terms of $\left.\epsilon<\kappa^{-0.1}, \epsilon_{1}, \epsilon_{2}, \delta_{0}, \frac{1}{q}, \frac{1}{q_{0}} \sim b_{1}-0.5\right)$ of $N_{j}$ and finally we multiply by $N_{(1)}^{\frac{\alpha}{q_{0}}+\frac{1}{q}+\theta+100 \epsilon+3 \epsilon_{1}+3 \epsilon_{2}}$ to the output.

In summary, by Algorithm 3, it suffices to establish the bound

$$
\mathcal{U}_{L_{1}, L_{2}, L_{3}}^{N_{1}, N_{2}, N_{3}} \leq K_{3}\left(N_{1}, N_{2}, N_{3}\right)
$$


where

$$
\mathcal{U}_{L_{1}, L_{2}, L_{3}}^{N_{1}, N_{2}, N_{3}}=\left(\sum_{k}\left|\sum_{\left(k_{1}, k_{2}, k_{3}\right) \in \Gamma(k)} a_{1}\left(k_{1}\right) \bar{a}_{2}\left(k_{2}\right) a_{3}\left(k_{3}\right)\right|^{2}\right)^{\frac{1}{2}},
$$

with $a_{1}, a_{2}, a_{3}$ having characterized parameters $\left(N_{1}, L_{1}\right),\left(N_{2}, L_{2}\right),\left(N_{3}, L_{3}\right)$, with respectively, satisfying corresponding estimates 8.10, 8.11 and 8.12 . Then the output is

$$
\text { Output of Algorithm } 3 \leq R N_{(1)}^{\frac{\alpha}{q_{0}}+\frac{1}{q}+\theta+100\left(\kappa^{-0.1}+\epsilon_{1}+\epsilon_{2}\right)} K_{3}\left(N_{1}, N_{2}, N_{3}\right) .
$$

\section{-Algorithm 4. Prototype: all of type (D)}

In this case, the simple algorithm is to obtain an estimate of the type

$$
\left\|\mathcal{M}_{L_{1}, L_{2}, L_{3}}^{N_{1}, N_{2}, N_{3}}\right\|_{l_{k}^{2}} \leq K\left(N_{1}, N_{2}, N_{3}, L_{1}, L_{2}, L_{3}\right) \prod_{j=1}^{3}\left\|\widetilde{w}_{j}\left(\lambda_{j}, k_{j}\right)\right\|_{l_{k_{j}}^{2}}
$$

and then take the $L_{\lambda_{1}, \lambda_{2}, \lambda_{3}}^{2} L_{\mu_{0} *}^{q_{0}}$ norm.

In summary, by Algorithm 4, it suffices to establish the bound

$$
\mathcal{U}_{L_{1}, L_{2}, L_{3}}^{N_{1}, N_{2}, N_{3}} \leq K_{4}\left(N_{1}, N_{2}, N_{3}\right)
$$

where

$$
\mathcal{U}_{L_{1}, L_{2}, L_{3}}^{N_{1}, N_{2}, N_{3}}=\left(\sum_{k}\left|\sum_{\left(k_{1}, k_{2}, k_{3}\right) \in \Gamma(k)} a_{1}\left(k_{1}\right) \bar{a}_{2}\left(k_{2}\right) a_{3}\left(k_{3}\right)\right|^{2}\right)^{\frac{1}{2}},
$$

with $a_{1}, a_{2}, a_{3}$ having characterized parameters $\left(N_{1}, L_{1}\right),\left(N_{2}, L_{2}\right),\left(N_{3}, L_{3}\right)$, with respectively, satisfying corresponding estimates (8.11). Then the output is

$$
\text { Output of Algorithm } 4 \leq R N_{(1)}^{\frac{\alpha}{q_{0}}+\theta+100\left(\kappa^{-0.1}+\epsilon_{1}+\epsilon_{2}\right)} K_{4}\left(N_{1}, N_{2}, N_{3}\right) .
$$

Remark 8.1. Note that Algorithm 3 and Algorithm 4 are purely deterministic, the only difference is that the upper bound $K_{3}\left(N_{1}, N_{2}, N_{3}\right)$ is formed by one $l_{k_{j}}^{\infty} l_{k_{j}^{*}}^{2}$ norm and two $l_{k_{j} k_{j}^{*}}^{2}$ norms, while the upper bound $K_{4}\left(N_{1}, N_{2}, N_{3}\right)$ is formed by three $l_{k_{j} k_{j}^{*}}^{2}$ norms.

\section{TRI-LinEAR ESTIMATES 1: HIGH-HIGH-HIGH INTERACTIONS}

In this section, we will prove (8) of Proposition 3.7 and (1) of Proposition 3.7 in the case $N_{1} \sim N_{2} \sim N_{3}$.

9.1. Diagonal terms. For $v_{1}, v_{2}, v_{3}$ of type $(\mathrm{G}),(\mathrm{C})$ or $(\mathrm{D})$ with characterized parameters $\left(N_{1}, L_{1}\right),\left(N_{2}, L_{2}\right)$ and $\left(N_{3}, L_{3}\right)$, note that

$$
\begin{aligned}
& \mathcal{F}_{t, x}\left(\chi(t) \mathcal{N}_{0}\left(v_{1}, v_{2}, v_{3}\right)\right)\left(\lambda+|k|^{\alpha}, k\right) \\
= & \int \widehat{\chi}\left(\lambda-\lambda_{1}+\lambda_{2}-\lambda_{3}\right) \widetilde{v}_{1}\left(\lambda_{1}, k\right) \overline{\widetilde{v}}_{2}\left(\lambda_{2}, k\right) \widetilde{v}_{3}\left(\lambda_{3}, k\right) d \lambda_{1} d \lambda_{2} d \lambda_{3} .
\end{aligned}
$$

Our goal is to show that

$$
\begin{aligned}
\mathcal{M} & :=\left\|\langle\lambda\rangle^{b_{1}-1} \int \widehat{\chi}\left(\lambda-\lambda_{1}+\lambda_{2}-\lambda_{3}\right) \widetilde{v}_{1}\left(\lambda_{1}, k\right) \overline{\widetilde{v}}_{2}\left(\lambda_{2}, k\right) \widetilde{v}_{3}\left(\lambda_{3}, k\right) d \lambda_{1} d \lambda_{2} d \lambda_{3}\right\|_{L_{\lambda}^{2} l_{n}^{2}} \\
& \lesssim N_{(1)}^{-s} N_{(2)}^{-\delta_{0}} .
\end{aligned}
$$

Note that we will omit the similar argument to treat $\Pi_{N_{0}}^{\perp} \mathcal{I} \mathcal{N}_{0}\left(v_{1}, v_{2}, v_{3}\right)$ when $N_{0} \gg N_{(1)}$. For $r_{j} \in\{2, q\}$, we denote by

$$
V_{j}^{\left(r_{j}\right)}\left(\lambda_{j}, k\right)=\left\langle\lambda_{j}\right\rangle^{\frac{2 b_{0}}{r_{j}^{\prime}}} \widetilde{v}_{j}\left(\lambda_{j}, k\right), f_{j}\left(\lambda_{j}\right)=\left\|V_{j}^{(2)}\left(\lambda_{j}, k\right)\right\|_{l_{k_{j}}^{2}}, \text { and } g_{j}\left(\lambda_{j}\right)=\left\|V_{j}^{(q)}\left(\lambda_{j}, k\right)\right\|_{l_{k}^{q}} .
$$


Note that when $v_{j}$ is of type $(\mathrm{G})$ or $(\mathrm{C})$, the spatial Fourier support is constraint on $\left|k_{j}\right| \leq N_{j}$, thus

$$
\left\|g_{j}\left(\lambda_{j}\right)\right\|_{L_{\lambda_{j}}^{q}} \leq N_{j}^{\frac{1}{q}}\left\|v_{j}\right\|_{X_{\infty, q}^{0, \frac{2 b_{0}}{q^{\prime}}}} .
$$

-Case 1: at least one of $v_{1}, v_{2}, v_{3}$ is of type (D)

First we assume that $v_{1}, v_{2}, v_{3}$ are all of type (D). From the embedding $l^{2} \hookrightarrow l^{\infty}$, Hölder's inequality and Lemma 2.5, we have

$$
\begin{aligned}
\mathcal{M} & \lesssim \int \frac{f_{1}\left(\lambda_{1}\right) f_{2}\left(\lambda_{2}\right) f_{3}\left(\lambda_{3}\right)}{\left\langle\lambda_{1}-\lambda_{2}+\lambda_{3}\right\rangle^{1-b_{1}}\left\langle\lambda_{1}\right\rangle^{b_{0}}\left\langle\lambda_{2}\right\rangle^{b_{0}}\left\langle\lambda_{3}\right\rangle^{b_{0}}} d \lambda_{1} d \lambda_{2} d \lambda_{3} \\
& \lesssim \prod_{j=1}^{3}\left\|f_{j}\left(\lambda_{j}\right)\right\|_{L_{\lambda_{j}}^{2}}=\prod_{j=1}^{3}\left\|v_{j}\right\|_{X^{0, b_{0}}} \lesssim\left(N_{1} N_{2} N_{3}\right)^{-s},
\end{aligned}
$$

which is conclusive.

Next, assume that exact one of $v_{1}, v_{2}, v_{3}$ is of type $(\mathrm{G})$ or $(\mathrm{C})$, say, $v_{1}$ is of type $(\mathrm{G})$ or (C), then from the same argument together with the embedding $l^{q} \hookrightarrow l^{\infty}$, we have

$$
\begin{aligned}
\mathcal{M} & =\left\|\langle\lambda\rangle^{b_{1}-1} \int \widehat{\chi}\left(\lambda-\lambda_{1}+\lambda_{2}-\lambda_{3}\right) \frac{V_{1}^{(q)}\left(\lambda_{1}, k\right) \bar{V}_{2}^{(2)}\left(\lambda_{2}, k\right) V_{3}^{(2)}\left(\lambda_{3}, k\right)}{\left\langle\lambda_{1}\right\rangle^{\frac{2 b_{0}}{q^{\prime}}}\left\langle\lambda_{2}\right\rangle^{b_{0}}\left\langle\lambda_{3}\right\rangle^{b_{0}}} d \lambda_{1} d \lambda_{2} d \lambda_{3}\right\|_{L_{\lambda}^{2} l_{k}^{2}} \\
& \lesssim \int \frac{g_{1}\left(\lambda_{1}\right) f_{2}\left(\lambda_{2}\right) f_{3}\left(\lambda_{3}\right)}{\left\langle\lambda_{1}-\lambda_{2}+\lambda_{3}\right\rangle^{1-b_{1}}\left\langle\lambda_{1}\right\rangle^{\frac{2 b_{0}}{q^{\prime}}}\left\langle\lambda_{2}\right\rangle^{b_{0}}\left\langle\lambda_{3}\right\rangle^{b_{0}}} d \lambda_{1} d \lambda_{2} d \lambda_{3} \\
& \lesssim\left\|g_{1}\left(\lambda_{1}\right)\right\|_{L_{\lambda_{1}}^{q}}\left\|f_{2}\left(\lambda_{2}\right)\right\|_{L_{\lambda_{2}}^{2}}\left\|f_{3}\left(\lambda_{3}\right)\right\|_{L_{\lambda_{3}}^{2}} \\
& \leq N_{1}^{\frac{1}{q}}\left\|v_{1}\right\|_{\substack{0, \frac{2 b_{0}}{q^{\prime}} \\
X_{\infty}, q}}\left\|v_{2}\right\|_{X^{0, b_{0}}}\left\|v_{3}\right\|_{X^{0, b_{0}}} \lesssim N_{1}^{-\frac{\alpha}{2}+\frac{1}{q}+\epsilon_{2}} N_{2}^{-s} N_{3}^{-s},
\end{aligned}
$$

which is conclusive.

If there are exact two of $v_{1}, v_{2}, v_{3}$ of type $(\mathrm{G})$ or $(\mathrm{C})$ and the other is of type $(\mathrm{D})$, the estimate is similar and we obtain that

$$
\mathcal{M} \lesssim N_{(1)}^{-s}\left(N_{(2)} N_{(3)}\right)^{-\frac{\alpha}{2}+\frac{1}{q}+\epsilon_{2}},
$$

which is also conclusive.

-Case 2: $v_{1}, v_{2}, v_{3}$ are all of type $(\mathbf{G})$ or $(\mathbf{C})$

Without loss of generality, we may assume that $N_{1} \geq N_{2} \geq N_{3}$ and we will put the $X^{0, b}$ norm on $v_{3}$. From the same manipulation as in the previous cases, we have

$$
\begin{aligned}
& \mathcal{M} \lesssim \int \frac{g_{1}\left(\lambda_{1}\right) g_{2}\left(\lambda_{2}\right) f_{3}\left(\lambda_{3}\right)}{\left\langle\lambda_{1}-\lambda_{2}+\lambda_{3}\right\rangle^{1-b_{1}}\left\langle\lambda_{1}\right\rangle^{\frac{2 b_{0}}{q^{\prime}}}\left\langle\lambda_{2}\right\rangle^{\frac{2 b_{0}}{q^{\prime}}}\left\langle\lambda_{3}\right\rangle^{b_{0}}} d \lambda_{1} \lambda_{2} d \lambda_{3} \\
& \lesssim\left\|g_{1}\left(\lambda_{1}\right)\right\|_{L_{\lambda_{1}}^{q}}\left\|g_{2}\left(\lambda_{2}\right)\right\|_{L_{\lambda_{2}}^{q}}\left\|f_{3}\left(\lambda_{3}\right)\right\|_{L_{\lambda_{3}}^{2}} \\
& \leq\left(N_{1} N_{2}\right)^{\frac{1}{q}}\left\|v_{1}\right\|_{\substack{0, \frac{2 b_{0}}{q^{q}} \\
\mathcal{q}_{\infty}}}\left\|v_{2}\right\|_{\substack{X_{\infty, q} \\
0, \frac{2 b_{0}}{q^{\prime}}}}\left\|v_{2}\right\|_{X^{0, b_{0}}} \lesssim\left(N_{1} N_{2}\right)^{-\frac{\alpha}{2}+\frac{1}{q}+\epsilon_{2}} N_{3}^{-s},
\end{aligned}
$$

which is conclusive. The proof of (8) of Proposition 3.7 is complete.

9.2. Non-diagonal terms. We assume that $N_{1} \sim N_{2} \sim N_{3} \sim N$. By Lemma 2.1 and the $X^{0, b}$-mapping property (Lemma 5.1 , outside a set of probability $<\mathrm{e}^{-N^{\theta} R^{2}}$, we have

$$
\left\|\mathcal{I N}_{3}\left(v_{1}, v_{2}, v_{3}\right)\right\|_{X^{0, b_{1}}} \lesssim R N^{-\delta_{0}}\left\|v_{j}\right\|_{X^{0, \frac{3}{8}}}
$$

for all $j \in\{1,2,3\}$, provided that the range of $\alpha$ satisfies

$$
\alpha-1+2 \nu s>s .
$$


Therefore, if at least one $v_{j}$ is of type (D), the right side can be bounded by $R N^{-s-\delta_{0}}$. If at least one $v_{j}$ is of type $(\mathrm{C})$, we have the upper bound

$$
R N^{-\delta_{0}-2(\alpha-1)+2 \epsilon_{1}}<R N^{-s-\delta_{0}},
$$

which is also conclusive from the hypothesis on the range of $\alpha$.

The only case left to treat alternatively is that when $v_{1}, v_{2}, v_{3}$ are all of type $(\mathrm{G})$. From Algorithm 1 in Section 8, it suffices to estimate

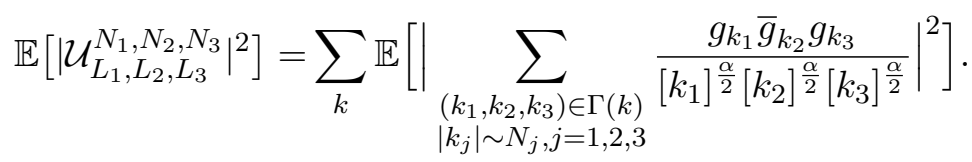

Recall that if the output of this estimate if $K_{1}\left(N_{1}, N_{2}, N_{3}\right)$, then outside of set of probability $<\mathrm{e}^{-N_{1}^{\theta} R^{\frac{2}{3}}}$,

$$
\left\|\mathcal{N}_{3}\left(v_{1}, v_{2}, v_{3}\right)\right\|_{X^{0, b_{1}}} \lesssim R N^{\frac{\alpha}{q_{0}}+\theta+100\left(\kappa^{-0.1}+\epsilon_{1}+\epsilon_{2}\right)} K_{1}\left(N_{1}, N_{2}, N_{3}\right)^{\frac{1}{2}}
$$

By expanding the square and using the independence, we have

$$
\begin{aligned}
\mathbb{E}\left[\left|\mathcal{U}_{L_{1}, L_{2}, L_{3}}^{N_{1}, N_{2}, N_{3}}\right|^{2}\right] & \sim\left(N_{1} N_{2} N_{3}\right)^{-\alpha} \sum_{\substack{k, k_{1}, k_{2}, k_{3} \\
\left(k_{1}, k_{2}, k_{3}\right) \in \Gamma(k)}} \prod_{j=1}^{3} \mathbf{1}_{\left|k_{j}\right| \sim N_{j}} \\
& \lesssim\left(N_{1} N_{2} N_{3}\right)^{-\alpha} \sum_{k, k_{2}, k_{3}} S_{k+k_{2}-k_{3}, k_{2}, k_{3}} \mathbf{1}_{\left|k+k_{2}-k_{3}\right| \sim N_{1}} \prod_{j=2}^{3} \mathbf{1}_{\left|k_{j}\right| \sim N_{j}} \\
& \lesssim\left(N_{1} N_{2} N_{3}\right)^{-\alpha} \sum_{k_{2}, k_{3}} N_{1}^{\epsilon}\left(1+\frac{N_{1}^{2-\alpha}}{\left\langle k_{2}-k_{3}\right\rangle}\right) \lesssim N_{1}^{-\alpha-2(\alpha-1)+\epsilon}
\end{aligned}
$$

thus $K_{1}\left(N_{1}, N_{2}, N_{3}\right)^{\frac{1}{2}} \sim N_{1}^{-\frac{\alpha}{2}-(\alpha-1)+\epsilon}$, which is conclusive by choosing $\epsilon<\kappa^{-0.1}$, say.

In summary, the proof of (1) of Proposition 3.7 in the case $N_{1} \sim N_{2} \sim N_{3}$ is complete.

\section{High-High-LOW INTERACTIONS}

In this section, we will prove (1) of Proposition 3.7 in the case $N_{(1)} \sim N_{(2)} \gg N_{(3)}$. More precisely, we will finish the estimate of $\left\|\mathcal{M}_{L_{1}, L_{2}, L_{3}}^{N_{1}, N_{2}, N_{3}}\right\|_{L_{\lambda_{1}}^{r_{1}} L_{\lambda_{2}}^{r_{2}} L_{\lambda_{3}}^{r_{3}} L_{\mu_{0} *}^{q_{0}} l_{k}^{2}}$ by executing one of Algorithm 1 to Algorithm 4, according to different triples of characterized parameters $\left(N_{j}, L_{j}\right), j=1,2,3$. Moreover, we will ignore all the small powers of $N_{j}$ in the estimates by assuming that

$$
S_{k_{1}, k_{2}, k_{3}}=\mathbf{1}_{\Phi_{k_{1}, k_{2}, k_{3}}=\mu_{0}+O(1)} \text { and } h_{k_{j} k_{j}^{*}}^{N_{j} L_{j}}=h_{k_{j} k_{j}^{*}}^{N_{j} L_{j}} \mathbf{1}_{\left|k_{j}-k_{j}^{*}\right| \leq L_{j}},
$$

since they are all compensated by a unified factor $N_{(1)}^{\frac{\alpha}{q_{0}}+\frac{3}{q}+\theta+100\left(\kappa^{-1}+\epsilon_{1}+\epsilon_{2}\right)}$. To save the notation, in this section, all the sums for $k, k_{j}$ are taken for $\left|k_{j}\right| \sim N_{j},|k| \lesssim N_{(1)}$ without declaration, when there is no risk of confusing.

10.1. The case $N_{1} \sim N_{2} \gg N_{3}$. First we deal with

- Case A-1: $a_{1}, a_{2}$ are all of type (D)

We execute Algorithm 4. By the triangle inequality and Cauchy-Schwartz,

$$
\begin{aligned}
\left|\mathcal{U}_{L_{1}, L_{2}, L_{3}}^{N_{1}, N_{2}, N_{3}}\right|^{2} & \leq\left(\sum_{k} \sum_{\left(k_{1}, k_{2}, k_{3}\right) \in \Gamma(k)}\left|a_{1}\left(k_{1}\right)\right|^{2}\left|a_{3}\left(k_{3}\right)\right|^{2} \mathbf{1}_{k_{1} k_{2}<0}\right) \cdot\left(\sup _{k} \sum_{\left(k_{1}, k_{2}, k_{3}\right) \in \Gamma(k)}\left|a_{2}\left(k_{2}\right)\right|^{2} \mathbf{1}_{k_{1} k_{2}<0}\right) \\
& +\left(\sum_{k} \sum_{\left(k_{1}, k_{2}, k_{3}\right) \in \Gamma(k)}\left|a_{1}\left(k_{1}\right)\right|^{2}\left|a_{3}\left(k_{3}\right)\right|^{2} \mathbf{1}_{k_{1} k_{2} \geq 0}\right) \cdot\left(\sup _{k} \sum_{\left(k_{1}, k_{2}, k_{3}\right) \in \Gamma(k)}\left|a_{2}\left(k_{2}\right)\right|^{2} \mathbf{1}_{k_{1} k_{2} \geq 0}\right) .
\end{aligned}
$$


Since $N_{3} \ll N_{1} \sim N_{2}$, for fixed $k$ and $k_{2}$,

$$
\sum_{\left(k_{1}, k_{2}, k_{3}\right) \in \Gamma(k)}\left|a_{2}\left(k_{2}\right)\right|^{2}=\sum_{k_{2}}\left|a_{2}\left(k_{2}\right)\right|^{2} \sum_{k_{3}} S_{k+k_{2}-k_{3}, k_{2}, k_{3}} \lesssim\left\|a_{2}\right\|_{l^{2}}^{2} .
$$

When $k_{1} k_{2}<0$, for fixed $k_{1}, k_{3}$,

$$
\left|\partial_{k_{2}} \Phi_{k_{1}, k_{2}, k_{3}}\right|=\left.\alpha\left|\operatorname{sign}\left(k_{2}\right)\right| k_{2}\right|^{\alpha-1}+\operatorname{sign}\left(k_{2}-k_{1}-k_{3}\right)\left|k_{2}-k_{1}-k_{3}\right|^{\alpha-1} \mid \gtrsim N_{1}^{\alpha-1},
$$

hence

$\sum_{k} \sum_{\left(k_{1}, k_{2}, k_{3}\right) \in \Gamma(k)}\left|a_{1}\left(k_{1}\right)\right|^{2}\left|a_{3}\left(k_{3}\right)\right|^{2} \mathbf{1}_{k_{1} k_{2}<0}=\sum_{k_{1}, k_{3}}\left|a_{1}\left(k_{1}\right)\right|^{2}\left|a_{3}\left(k_{3}\right)\right|^{2} \sum_{k_{2}} S_{k_{1}, k_{2}, k_{3}} \mathbf{1}_{k_{1} k_{2}<0} \lesssim\left\|a_{1}\right\|_{l^{2}}^{2}\left\|a_{3}\right\|_{l^{2}}^{2}$.

When $k_{1} k_{2} \geq 0$, if $\operatorname{sign}\left(k_{2}\right)=\operatorname{sign}\left(k_{2}-k_{1}-k_{3}\right)$, then $\left|\partial_{k_{2}} \Phi_{k_{1}, k_{2}, k_{3}}\right| \gtrsim N_{1}^{\alpha-1}$. Otherwise, we have for fixed $k_{1}, k_{3}$, by Lemma 2.9 ,

$$
\sum_{k_{2}} S_{k_{1}, k_{2}, k_{3}} \mathbf{1}_{k_{2}\left(k_{1}+k_{3}-k_{2}\right)>0} \lesssim N_{2}^{1-\frac{\alpha}{2}} \sim N_{1}^{1-\frac{\alpha}{2}}
$$

Therefore,

$$
\sum_{k} \sum_{\left(k_{1}, k_{2}, k_{3}\right) \in \Gamma(k)}\left|a_{1}\left(k_{1}\right)\right|^{2}\left|a_{3}\left(k_{3}\right)\right|^{2} \mathbf{1}_{k_{1} k_{2} \geq 0} \lesssim N_{1}^{1-\frac{\alpha}{2}}\left\|a_{1}\right\|_{l^{2}}^{2}\left\|a_{3}\right\|_{l^{2}}^{2}
$$

We have proved:

Proposition 10.1. When $N_{1} \sim N_{2} \gg N_{3}$ and $a_{1}, a_{2}$ are of type (D), the output of the Case A-1 is bounded by

$$
\mathcal{U}_{L_{1}, L_{2}, L_{3}}^{N_{1}, N_{2}, N_{3}} \lesssim N_{1}^{\frac{1}{2}-\frac{\alpha}{4}} N_{1}^{-2 s} N_{3}^{-\frac{\alpha-1}{2}}
$$

Note that the power $N_{3}^{-\frac{\alpha-1}{2}}$ comes from the worst case when $a_{3}$ is of type $(\mathrm{G})$.

- Case A-2: Exact one of $a_{1}, a_{2}$ is of type $(\mathrm{G})$ or $(\mathrm{C})$, the other is of type (D).

First we assume that $a_{1}$ is of type $(\mathrm{G})$ or $(\mathrm{C})$ and $a_{2}$ is of type (D). By Cauchy-Schwartz,

$$
\begin{aligned}
\left|\mathcal{U}_{L_{1}, L_{2}, L_{3}}^{N_{1}, N_{2}, N_{3}}\right|^{2} & \leq\left\|a_{1}\right\|_{l^{\infty}}^{2}\left(\sum_{\substack{k, k_{2}, k_{3} \\
\left|k_{2}\right| \sim N_{2} \\
\left|k+k_{2}-k_{3}\right| \sim N_{1}}}\left|a_{3}\left(k_{3}\right)\right|^{2} S_{k+k_{2}-k_{3}, k_{2}, k_{3}}\right) \sup _{k} \sum_{\substack{k_{2}, k_{3} \\
\left|k_{3}\right| \sim N_{3} \\
\left|k+k_{2}-k_{3}\right| \sim N_{1}}}\left|a_{2}\left(k_{2}\right)\right|^{2} S_{k+k_{2}-k_{3}, k_{2}, k_{3}} \\
& \lesssim\left\|a_{1}\right\|_{l^{\infty}}^{2}\left\|a_{2}\right\|_{l^{2}}^{2} \sum_{k_{1}, k_{2}, k_{3}}\left|a_{3}\left(k_{3}\right)\right|^{2} S_{k_{1}, k_{2}, k_{3}} \mathbf{1}_{\left|k_{2}\right| \sim N_{2}} \mathbf{1}_{\left|k_{1}\right| \sim N_{1}},
\end{aligned}
$$

since for fixed $k, k_{2}$

$$
\sum_{k_{3}} S_{k+k_{2}-k_{3}, k_{2}, k_{3}} \mathbf{1}_{\left|k_{3}\right| \sim N_{3}} \mathbf{1}_{\left|k+k_{2}-k_{3}\right| \sim N_{1}} \lesssim 1,
$$

due to the fact that $N_{3} \ll N_{1}$. Now for fixed $k_{2}, k_{3}$, if $k_{1}\left(k_{1}-k_{2}+k_{3}\right) \geq 0$, we have

$$
\left.\left|\partial_{k_{1}} \Phi_{k_{1}, k_{2}, k_{3}}\right| \sim\left|\operatorname{sign}\left(k_{1}\right)\right| k_{1}\right|^{\alpha-1}-\operatorname{sign}\left(k_{1}-k_{2}+k_{3}\right)\left|k_{1}-k_{2}+k_{3}\right|^{\alpha-1}\left|\gtrsim N^{\alpha-2}\right| k_{2}-k_{3} \mid,
$$

otherwise $\left|\partial_{k_{1}} \Phi_{k_{1}, k_{2}, k_{3}}\right| \gtrsim N_{1}^{\alpha-1}$, hence

$$
\sum_{k_{1}} S_{k_{1}, k_{2}, k_{3}} \mathbf{1}_{\left|k_{1}\right| \sim N_{1},\left|k_{3}\right| \sim N_{3}} \mathbf{1}_{\left|k_{2}\right| \sim N_{2}} \lesssim 1+\frac{N_{1}^{2-\alpha}}{\left\langle k_{2}-k_{3}\right\rangle} .
$$

Therefore, modulo a factor $N_{1}^{\epsilon}$, we have

$$
\left|\mathcal{U}_{L_{1}, L_{2}, L_{3}}^{N_{1}, N_{2}, N_{3}}\right| \lesssim\left\|a_{1}\right\|_{l^{\infty}}\left\|a_{2}\right\|_{l^{2}}\left\|a_{3}\right\|_{l^{2}}\left(N_{2}^{\frac{1}{2}}+N_{1}^{1-\frac{\alpha}{2}}\right) \lesssim N_{1}^{-\frac{\alpha-1}{2}-s} L_{1}^{-\nu} N_{3}^{-\frac{\alpha-1}{2}} .
$$


Next we assume that $a_{1}$ is of type (D) and $a_{2}$ is of type (G) or (C). Repeating the argument above, we have

$$
\begin{aligned}
\left|\mathcal{U}_{L_{1}, L_{2}, L_{3}}^{N_{1}, N_{2}, N_{3}}\right|^{2} & \leq\left\|a_{2}\right\|_{l^{\infty}}^{2}\left(\sum_{\substack{k, k_{1}, k_{3} \\
\left|k_{1}\right| \sim N_{1}}}\left|a_{3}\left(k_{3}\right)\right|^{2} S_{k_{1}, k_{1}+k_{3}-k, k_{3}}\right) \sup _{k} \sum_{\substack{k_{1}, k_{3} \\
\left|k_{3}\right| \sim N_{3} \\
\left|k_{1}+k_{3}-k\right| \sim N_{2}}}\left|a_{1}\left(k_{1}\right)\right|^{2} S_{k_{1}, k_{1}+k_{3}-k, k_{3}} \\
& \lesssim\left\|a_{1}\right\|_{l^{2}}^{2}\left\|a_{2}\right\|_{l^{\infty}}^{2} \sum_{k_{1}, k_{2}, k_{3}}\left|a_{3}\left(k_{3}\right)\right|^{2} S_{k_{1}, k_{2}, k_{3}} \mathbf{1}_{\left|k_{1}\right| \sim N_{1}} \mathbf{1}_{\left|k_{2}\right| \sim N_{2}} \\
& \lesssim\left\|a_{1}\right\|_{l^{2}}^{2}\left\|a_{2}\right\|_{l^{\infty}}^{2}\left\|a_{3}\right\|_{l^{2}}^{2}\left(N_{2}+N_{1}^{2-\alpha}\right) \lesssim\left\|a_{1}\right\|_{l^{2}}^{2}\left\|a_{2}\right\|_{l^{\infty}}^{2}\left\|a_{3}\right\|_{l^{2}}^{2} N_{2},
\end{aligned}
$$

thanks to the fact that $N_{3} \ll N_{2} \sim N_{1}$. Therefore, we have proved:

Proposition 10.2. Assume that $N_{1} \sim N_{2} \gg N_{3}$ and exact one of $a_{1}, a_{2}$ is of type $(G)$ or (C) and the other is of type (D). Then modulo a factor $N_{1}^{\epsilon}, \epsilon<\kappa^{-0.1}$, the output of the Case A-1 is bounded by

$$
\mathcal{U}_{L_{1}, L_{2}, L_{3}}^{N_{1}, N_{2}, N_{3}} \lesssim N_{1}^{-\frac{\alpha-1}{2}-s} L_{1}^{-\nu} N_{3}^{-\frac{\alpha-1}{2}},
$$

if $a_{1}$ is of type $(C)$ or $(G)$ and $a_{2}$ is of type (D). If $a_{1}$ is of type (D) and $a_{2}$ is of type $(C)$ or $(G)$, modulo a factor $N_{1}^{\epsilon}, \epsilon<\kappa^{-0.1}$, we have

$$
\mathcal{U}_{L_{1}, L_{2}, L_{3}}^{N_{1}, N_{2}, N_{3}} \lesssim N_{1}^{-\frac{\alpha-1}{2}-s} L_{2}^{-\nu} N_{3}^{-\frac{\alpha-1}{2}} .
$$

-Case A-3: $a_{1}, a_{2}$ are all of type (G) or (C)

If at least one of $a_{1}, a_{2}$ is of type (C), we first execute Algorithm 4. Without loss of generality, we may assume that $a_{1}$ is of type (C) and $L_{1} \geq L_{2}$. From the same argument as for Case A-2, we have

$$
\left|\mathcal{U}_{L_{1}, L_{2}, L_{3}}^{N_{1}, N_{2}, N_{3}}\right| \lesssim N_{2}^{\frac{1}{2}}\left\|a_{1}\right\|_{l^{\infty}}\left\|a_{2}\right\|_{l^{2}}\left\|a_{3}\right\|_{l^{2}}
$$

If $a_{2}$ is of type $(\mathrm{G})$, we have

$$
N_{2}^{\frac{1}{2}}\left\|a_{1}\right\|_{l^{\infty}}\left\|a_{2}\right\|_{l^{2}}\left\|a_{3}\right\|_{l^{2}} \lesssim N_{1}^{-(\alpha-1)} L_{1}^{-\nu} N_{3}^{-\frac{\alpha-1}{2}},
$$

since the worst case is that $a_{3}$ is of type $(\mathrm{G})$. If $a_{2}$ is of type $(\mathrm{C})$, we have

$$
N_{2}^{\frac{1}{2}}\left\|a_{1}\right\|_{l^{\infty}}\left\|a_{2}\right\|_{l^{2}}\left\|a_{3}\right\|_{l^{2}} \lesssim N_{1}^{-\frac{3(\alpha-1)}{2}} L_{1}^{-\nu} L_{2}^{-\nu} N_{3}^{-\frac{\alpha-1}{2}} .
$$

In order to get a better bound when $L_{1} \vee L_{2}$ is relatively small, we need to execute Algorithm 2. In what follows, we do not distinguish the type (G) and (C), since we will only use the $S^{b, q}$ norm of $h^{N_{j} L_{j}}$, which does not make any difference between type $(\mathrm{G})$ and (C) terms. Fix $L=10\left(L_{1} \vee L_{2}\right)$, recall the definition 8.8 of $\eta_{k, k^{\prime}}^{(3)}$, we have

$$
\begin{aligned}
& \sum_{k, k^{\prime}:\left|k-k^{\prime}\right| \geq L}\left|\eta_{k, k^{\prime}}^{(3)}\right|^{2} \\
= & \sum_{\substack{k, k^{\prime} \\
\left|k-k^{\prime}\right| \geq L}}\left|\sum_{\substack{\left(k_{1}, k_{2}, k_{3}\right) \in \Gamma(k) \\
\left(k_{1}^{\prime}, k_{2}^{\prime}, k_{3}\right) \in \Gamma\left(k^{\prime}\right) \\
k_{1}^{*}, k_{1}^{\prime *}, k_{2}^{*}, k_{2}^{\prime *}}} h_{k_{1} k_{1}^{*}} \bar{h}_{k_{1}^{\prime} k_{1}^{\prime *}} \bar{h}_{k_{2} k_{2}^{*}} h_{k_{2}^{\prime} k_{2}^{\prime *}} S_{k_{1}, k_{2}, k_{3}} S_{k_{1}^{\prime}, k_{2}^{\prime}, k_{3}} \frac{g_{k_{1}^{*}} \bar{g}_{k_{1}^{\prime *}} \bar{g}_{k_{2}^{*}} g_{k_{2}^{\prime *}}}{\left[k_{1}^{*}\right]^{\frac{\alpha}{2}}\left[k_{1}^{\prime *}\right]^{\frac{\alpha}{2}}\left[k_{2}^{*}\right]^{\frac{\alpha}{2}}\left[k_{2}^{\prime *}\right]^{\frac{\alpha}{2}}}\right|^{2} .
\end{aligned}
$$

To simplify the summation before expanding the square, we split the inner $\operatorname{sum} \sum_{k_{1}^{*}, k_{1}^{\prime *}, k_{2}^{*}, k_{2}^{\prime *}}$ into six groups:

(1) $k_{1}^{*}=k_{1}^{\prime *}, k_{2}^{*}=k_{2}^{\prime *}$

(2) $k_{1}^{*}=k_{2}^{*}, k_{1}^{* *}=k_{2}^{\prime *}$;

(3) $k_{1}^{*}=k_{1}^{\prime *}, k_{2}^{*} \neq k_{2}^{\prime *}$;

(4) $k_{1}^{*} \neq k_{1}^{\prime *}, k_{2}^{*}=k_{2}^{\prime *}$;

(5) $k_{1}^{*}=k_{2}^{*}, k_{1}^{\prime *} \neq k_{2}^{\prime *}$ or $k_{1}^{\prime *}=k_{2}^{\prime *}, k_{1}^{*} \neq k_{2}^{*}$;

(6) No pairings in $k_{1}^{*}, k_{1}^{\prime *}, k_{2}^{*}, k_{2}^{\prime *}$ (in the sense that $k_{1}^{*} \neq k_{1}^{\prime *}, k_{2}^{*}$ and $k_{2}^{*} \neq k_{2}^{\prime *}, k_{1}^{*}$ ). 
Taking the expectation, the contributions from $(j)(j \in\{1,2,3,4,5,6\})$ can be bounded by

$$
\begin{aligned}
& \mathcal{C}_{j}:=N_{1}^{-4 \alpha} \sum_{\substack{k, k^{\prime} \\
\left|k-k^{\prime}\right| \geq L}} \sum_{\substack{\left(k_{1}, k_{2}, k_{3}\right) \in \Gamma(k) \\
\left(k_{1}^{\prime}, k_{2}^{\prime}, k_{3}\right) \in \Gamma\left(k^{\prime}\right)}} \prod_{\substack{\left(m_{1}, m_{2}, m_{3}\right) \in \Gamma(k) \\
\left(m_{1}^{\prime}, m_{2}^{\prime}, m_{3}\right) \in \Gamma\left(k^{\prime}\right)}}^{2}\left\|h_{k_{j} k_{j}^{*}}\right\|_{l_{k_{j}^{*}}^{2 *}}\left\|h_{m_{j} m_{j}^{*}}\right\|_{l_{m_{j}^{*}}^{2}}\left\|h_{k_{j}^{\prime} k_{j}^{\prime *}}\right\|_{l_{k_{j}^{\prime *}}^{2}}\left\|h_{m_{j}^{\prime} m_{j}^{\prime *}}\right\|_{l_{m_{j}^{\prime *}}^{2}} \\
& \times S_{k_{1}, k_{2}, k_{3}} S_{k_{1}^{\prime}, k_{2}^{\prime}, k_{3}} S_{m_{1}, m_{2}, m_{3}} S_{m_{1}^{\prime}, m_{2}^{\prime}, m_{3}} \mathbf{1}_{\mathcal{A}_{j}}
\end{aligned}
$$

where $\mathcal{A}_{j}$ is the index set of $k_{1}, k_{2}, k_{3}, k_{1}^{\prime}, k_{2}^{\prime}, m_{1}, m_{2}, m_{3}, m_{1}^{\prime}, m_{2}^{\prime}, k, k^{\prime}$ defining by the constraints:

$$
\begin{aligned}
& \mathcal{A}_{1}:=\left\{\left|k_{i}-k_{i}^{\prime}\right| \leq 2 L_{i},\left|m_{i}-m_{i}^{\prime}\right| \leq 2 L_{i}, i=1,2\right\} ; \\
& \mathcal{A}_{2}:=\left\{\left|k_{1}-k_{2}\right|,\left|m_{1}-m_{2}\right| \leq 2 L_{1} \vee L_{2} ;\left|k_{1}^{\prime}-k_{2}^{\prime}\right|,\left|m_{1}^{\prime}-m_{2}^{\prime}\right| \leq 2 L_{1} \vee L_{2}\right\} ; \\
& \mathcal{A}_{3}:=\left\{\left|k_{1}-k_{1}^{\prime}\right|,\left|m_{1}-m_{1}^{\prime}\right| \leq 2 L_{1},\left|k_{2}-m_{2}\right|,\left|k_{2}^{\prime}-m_{2}^{\prime}\right| \leq 2 L_{2}\right\} ; \\
& \mathcal{A}_{4}:=\left\{\left|k_{1}-m_{1}\right|,\left|k_{1}^{\prime}-m_{1}^{\prime}\right| \leq 2 L_{1},\left|k_{2}-k_{2}^{\prime}\right|,\left|m_{2}-m_{2}^{\prime}\right| \leq 2 L_{2}\right\} ; \\
& \mathcal{A}_{5}:=\left\{\left|k_{1}-k_{2}\right|,\left|m_{1}-m_{2}\right| \leq 2 L_{1} \vee L_{2},\left|k_{1}^{\prime}-m_{1}^{\prime}\right| \leq 2 L_{1},\left|k_{2}^{\prime}-m_{2}^{\prime}\right| \leq 2 L_{2}\right\} \\
& \cup\left\{\left|k_{1}^{\prime}-k_{2}^{\prime}\right|,\left|m_{1}^{\prime}-m_{2}^{\prime}\right| \leq 2 L_{1} \vee L_{2},\left|k_{1}-m_{1}\right| \leq 2 L_{1},\left|k_{2}-m_{2}\right| \leq 2 L_{2}\right\} ; \\
& \mathcal{A}_{6}:=\left\{\left|k_{1}-m_{1}\right|,\left|k_{1}^{\prime}-m_{1}^{\prime}\right| \leq 2 L_{1},\left|k_{2}-m_{2}\right|,\left|k_{2}^{\prime}-m_{2}^{\prime}\right| \leq 2 L_{2}\right\} \\
& \cup\left\{\left|k_{1}-m_{2}^{\prime}\right|,\left|k_{2}^{\prime}-m_{1}\right| \leq 2 L_{1} \vee L_{2},\left|k_{1}^{\prime}-m_{1}^{\prime}\right| \leq 2 L_{1},\left|k_{2}-m_{2}\right| \leq 2 L_{2}\right\} ; \\
& \cup\left\{\left|k_{1}^{\prime}-m_{2}\right|,\left|k_{2}-m_{1}^{\prime}\right| \leq 2 L_{1} \vee L_{2},\left|k_{1}-m_{1}\right| \leq 2 L_{1},\left|k_{2}^{\prime}-m_{2}^{\prime}\right| \leq 2 L_{2}\right\} \\
& \cup\left\{\left|k_{1}-m_{2}^{\prime}\right|,\left|k_{2}-m_{1}^{\prime}\right|,|| k_{1}^{\prime}-m_{2}|,| k_{2}^{\prime}-m_{1} \mid \leq 2 L_{1} \vee L_{2}\right\} .
\end{aligned}
$$

Note that under the constraint $\left|k-k^{\prime}\right| \geq L=10\left(L_{1} \vee L_{2}\right), \mathcal{A}_{1}=\mathcal{A}_{2}=\emptyset$, hence $\mathcal{C}_{1}=\mathcal{C}_{2}=0$. Indeed, on $\mathcal{A}_{1}$,

$$
\left|k-k^{\prime}\right|=\left|\left(k_{1}-k_{2}+k_{3}\right)-\left(k_{1}^{\prime}-k_{2}^{\prime}+k_{3}\right)\right| \leq\left|k_{1}-k_{1}^{\prime}\right|+\left|k_{2}-k_{2}^{\prime}\right| \leq 2 L_{1}+2 L_{2}<L .
$$

On $\mathcal{A}_{2}$,

$$
\left|k-k^{\prime}\right| \leq\left|k_{1}-k_{2}\right|+\left|k_{1}^{\prime}-k_{2}^{\prime}\right| \leq 4\left(L_{1} \vee L_{2}\right)<L .
$$

On $\mathcal{A}_{3},\left|k-k^{\prime}+k_{2}-k_{2}^{\prime}\right| \leq 2 L_{1},\left|k-k^{\prime}+m_{2}-m_{2}^{\prime}\right| \leq 2 L_{1}$ and $\left|k_{2}-m_{2}\right| \leq 2 L_{2},\left|k_{2}^{\prime}-m_{2}^{\prime}\right| \leq 2 L_{2}$, we have

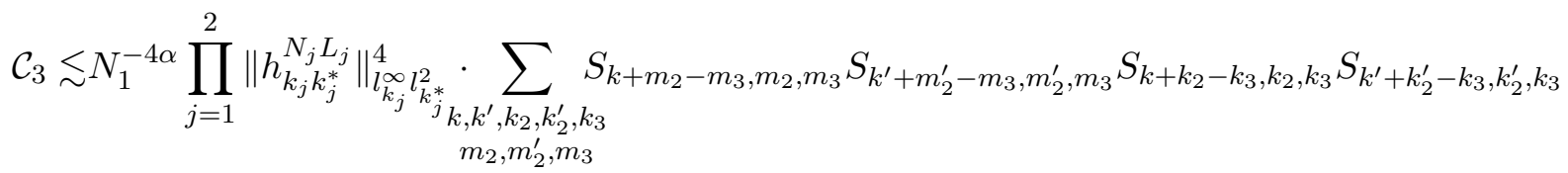

$$
\begin{aligned}
& \times \mathbf{1}_{\left|k-k^{\prime}+k_{2}-k_{2}^{\prime}\right| \leq 2 L_{1}} \mathbf{1}_{\left|k_{2}-m_{2}\right| \leq 2 L_{2}} \\
& \lesssim N_{1}^{-4 \alpha}\left(L_{1} L_{2}\right)^{-4 \nu} L_{2}^{2} \sum_{k, k^{\prime}, k_{2}, k_{2}^{\prime}, k_{3}} \mathbf{1}_{\left|k-k^{\prime}+k_{2}-k_{2}^{\prime}\right| \leq 2 L_{1}} S_{k+k_{2}-k_{3}, k_{2}, k_{3}} S_{k^{\prime}+k_{2}^{\prime}-k_{3}, k_{2}^{\prime}, k_{3}},
\end{aligned}
$$

where we first use

$$
\sum_{m_{3}} S_{k+m_{2}-m_{3}, m_{2}, m_{3}} \lesssim 1
$$

and then sum for $\mathbf{1}_{\left|k_{2}-m_{2}\right| \leq 2 L_{2},\left|k_{2}^{\prime}-m_{2}^{\prime}\right| \leq 2 L_{2}}$ over $m_{2}, m_{2}^{\prime}$. Next we sum over $k^{\prime}$ first and estimate (modulo a small factor $N_{1}^{\epsilon}$ )

$$
\begin{aligned}
& \sum_{k, k^{\prime}, k_{2}, k_{2}^{\prime}, k_{3}} \mathbf{1}_{\left|k-k^{\prime}+k_{2}-k_{2}^{\prime}\right| \leq 2 L_{1}} S_{k+k_{2}-k_{3}, k_{2}, k_{3}} S_{k^{\prime}+k_{2}^{\prime}-k_{3}, k_{2}^{\prime}, k_{3}} \\
\leq & 2 L_{1} \sum_{k_{2}, k_{3}} \sum_{k_{2}^{\prime}} \sum_{k} S_{k+k_{2}-k_{3}, k_{2}, k_{3}} \\
\lesssim & L_{1} N_{2} \sum_{k_{2}, k_{3}}\left(1+\frac{N_{1}^{2-\alpha}}{\left\langle k_{2}-k_{3}\right\rangle}\right) \lesssim L_{1} N_{2}\left(N_{2} N_{3}+N_{1}^{2-\alpha} N_{3}\right) \lesssim L_{1} N_{1}^{2} N_{3} .
\end{aligned}
$$


Therefore,

$$
\mathcal{C}_{3} \lesssim L_{1}^{1-4 \nu} L_{2}^{2-4 \nu} N_{1}^{2-4 \alpha} N_{3}
$$

Note that in the estimate of $\mathcal{C}_{3}$, we do not make use of the constraint $\left|k-k^{\prime}\right| \geq L$.

The estimate for $\mathcal{C}_{4}$ is similar as for $\mathcal{C}_{3}$, by switching $k_{2}, k_{2}^{\prime}, m_{2}, m_{2}^{\prime}$ to $k_{1}, k_{1}^{\prime}, m_{1}, m_{1}^{\prime}$. More precisely,

$$
\begin{gathered}
\mathcal{C}_{4} \lesssim N_{1}^{-4 \alpha} \prod_{j=1}^{2}\left\|h_{k_{j} k_{j}^{*}}^{N_{j} L_{j}}\right\|_{l_{k_{j}}^{\infty} l_{k_{j}^{*}}^{2}}^{4} \cdot \sum_{\substack{k, k^{\prime}, k_{1}, k_{1}^{\prime}, k_{3} \\
m_{1}, m_{1}^{\prime}, m_{3}}} S_{k_{1}, m_{1}+m_{3}-k, m_{3}} S_{m_{1}^{\prime}, m_{1}^{\prime}+m_{3}-k^{\prime}, m_{3}} S_{k_{1}, k_{1}+k_{3}-k, k_{3}} S_{k_{1}^{\prime}, k_{1}^{\prime}+k_{3}-k^{\prime}, k_{3}} \\
\times \mathbf{1}_{\substack{\left|k_{1}-k_{1}^{\prime}+k^{\prime}-k\right| \leq 2 L_{2} \\
\left|m_{1}-m_{1}^{\prime}+k^{\prime}-k\right| \leq 2 L_{2}}} \mathbf{1}_{\left|k_{1}-m_{1}\right| \leq 2 L_{1}}\left|k_{1}^{\prime}-m_{1}^{\prime}\right| \leq 2 L_{1} \\
\lesssim N_{1}^{-4 \alpha}\left(L_{1} L_{2}\right)^{-4 \nu} L_{1}^{2} \sum_{k, k^{\prime}, k_{1}, k_{1}^{\prime}, k_{3}} \mathbf{1}_{\left|k_{1}-k_{1}^{\prime}+k^{\prime}-k\right| \leq 2 L_{2}} S_{k_{1}, k_{1}+k_{3}-k, k_{3}} S_{k_{1}^{\prime}, k_{1}^{\prime}+k_{3}-k^{\prime}, k_{3},},
\end{gathered}
$$

where we used

$$
\sum_{m_{1}, m_{1}^{\prime}} \mathbf{1}_{\left|m_{1}-k_{1}\right| \leq 2 L_{1}} \sum_{\left|m_{1}^{\prime}-k_{1}^{\prime}\right| \leq 2 L_{1}} S_{m_{3}} S_{m_{1}, m_{1}+m_{3}-k, m_{3}} \lesssim L_{1}^{2}
$$

Next we sum over $k^{\prime}$ first and estimate (modulo a factor $N_{1}^{\epsilon}$ )

$$
\begin{gathered}
\sum_{k, k^{\prime}, k_{1}, k_{1}^{\prime}, k_{3}} \mathbf{1}_{\left|k_{1}-k_{1}^{\prime}+k^{\prime}-k\right| \leq 2 L_{2}} S_{k_{1}, k_{1}+k_{3}-k, k_{3}} S_{k_{1}^{\prime}, k_{1}^{\prime}+k_{3}-k^{\prime}, k_{3}} \\
\lesssim L_{2} \sum_{k, k_{3}} \sum_{k_{1}^{\prime}} \sum_{k_{1}} S_{k_{1}, k_{1}+k_{3}-k, k_{3}} \lesssim L_{2} N_{1} \sum_{k, k_{3}}\left(1+\frac{N_{1}^{2-\alpha}}{\left\langle k-k_{3}\right\rangle}\right) \lesssim L_{2} N_{1}^{2} N_{3} .
\end{gathered}
$$

Thus

$$
\mathcal{C}_{4} \lesssim L_{2}^{1-4 \nu} L_{1}^{2-4 \nu} N_{1}^{2-4 \alpha} N_{3}
$$

To estimate $\mathcal{C}_{5}$, by symmetry,

$$
\begin{aligned}
& \mathcal{C}_{5} \lesssim N_{1}^{-4 \alpha} \prod_{j=1}^{2}\left\|h_{k_{j} k_{j}^{*}}^{N_{j} L_{j}}\right\|_{\substack{l_{k_{j}} l_{k_{j}^{*}}^{*} \\
\text { s. } \\
k, k^{\prime}, k_{2}, k_{2}^{\prime}, k_{3} \\
m_{2}, m_{2}^{\prime}, m_{3}}} S_{k+m_{2}-m_{3}, m_{2}, m_{3}} S_{k^{\prime}+m_{2}^{\prime}-m_{3}, m_{2}^{\prime}, m_{3}} S_{k+k_{2}-k_{3}, k_{2}, k_{3}} S_{k^{\prime}+k_{2}^{\prime}-k_{3}, k_{2}^{\prime}, k_{3}} \\
& \times \mathbf{1}_{\left|k-k_{3}\right| \leq 2 L_{1} \vee L_{2}} \mathbf{1}_{\left|k-m_{3}\right| \leq 2 L_{1} \vee L_{2}}-m_{2}^{\prime} \mid \leq 2 L_{2} .
\end{aligned}
$$

First we sum over $m_{2}$ and obtain that

$$
\sum_{m_{2}} S_{k+m_{2}-m_{3}, m_{2}, m_{3}} \mathbf{1}_{\left|k-m_{3}\right| \leq 2 L_{1} \vee L_{2}} \lesssim \frac{N_{1}^{2-\alpha}}{\left\langle k-m_{3}\right\rangle} \mathbf{1}_{\left|k-m_{3}\right| \leq 2 L_{1} \vee L_{2}},
$$

since $L_{1} \vee L_{2} \ll N_{1}^{2-\alpha}$. Next we sum over $m_{2}^{\prime}$ and then $m_{3}$ to obtain that

$$
\mathcal{C}_{5} \lesssim N_{1}^{-4 \alpha}\left(L_{1} L_{2}\right)^{-4 \nu} L_{2} \sum_{k, k^{\prime}, k_{2}, k_{2}^{\prime}, k_{3}} N_{1}^{2-\alpha} \mathbf{1}_{\left|k-k_{3}\right| \leq 2 L_{1} \vee L_{2}} S_{k+k_{2}-k_{3}, k_{2}, k_{3}} S_{k^{\prime}+k_{2}^{\prime}-k_{3}, k_{2}^{\prime}, k_{3}} .
$$

Using

$$
\mathbf{1}_{\left|k-k_{3}\right| \leq 2 L_{1} \vee L_{2}} \sum_{k_{2}} S_{k+k_{2}-k_{3}, k_{2}, k_{3}} \lesssim \frac{N_{1}^{2-\alpha}}{\left\langle k-k_{3}\right\rangle} \mathbf{1}_{\left|k-k_{3}\right| \leq 2 L_{1} \vee L_{2}}
$$

and

$$
\sum_{k^{\prime}} S_{k^{\prime}+k_{2}^{\prime}-k_{3}, k_{2}^{\prime}, k_{3}} \lesssim 1+\frac{N_{1}^{2-\alpha}}{\left\langle k_{2}^{\prime}-k_{3}\right\rangle}
$$


we have (modulo a factor $N_{1}^{\epsilon}$ )

$$
\begin{aligned}
\mathcal{C}_{5} & \lesssim N_{1}^{-4 \alpha+2-\alpha} L_{1}^{-4 \nu} L_{2}^{1-4 \nu} \sum_{k, k_{2}^{\prime}, k_{3}} \mathbf{1}_{\left|k-k_{3}\right| \leq 2 L_{1} \vee L_{2}} \frac{N_{1}^{2-\alpha}}{\left\langle k-k_{3}\right\rangle}\left(1+\frac{N_{1}^{2-\alpha}}{\left\langle k_{2}^{\prime}-k_{3}\right\rangle}\right) \\
& \lesssim N_{1}^{-4 \alpha+2(2-\alpha)} L_{1}^{-4 \nu} L_{2}^{1-4 \nu}\left(N_{2} \sum_{k, k_{3}} \frac{1}{\left\langle k-k_{3}\right\rangle}+\sum_{k_{3}} \sum_{k} \frac{1}{\left\langle k-k_{3}\right\rangle} \sum_{k_{2}^{\prime}} \frac{N_{1}^{2-\alpha}}{\left\langle k_{2}^{\prime}-k_{3}\right\rangle}\right) \\
& \lesssim{ }_{\epsilon} N_{1}^{-4 \alpha+2(2-\alpha)} L_{1}^{-4 \nu} L_{2}^{1-4 \nu}\left(N_{2} N_{3}+N_{1}^{2-\alpha} N_{3}\right) .
\end{aligned}
$$

Thus

$$
\mathcal{C}_{5} \lesssim N_{1}^{5-6 \alpha} N_{3} L_{1}^{-4 \nu} L_{2}^{1-4 \nu}
$$

Next we estimate $\mathcal{C}_{6}$. We observe that whatever the pairing is, we can always do the sum $\sum_{m_{2}, m_{2}^{\prime}, m_{3}}$ first to obtain a factor $L_{1} L_{2}^{2}+L_{1}^{2} L_{2}$, hence

$$
\mathcal{C}_{6} \lesssim N_{1}^{-4 \alpha} \prod_{j=1}^{2}\left\|h_{k_{j} k_{j}^{*}}^{N_{j} L_{j}}\right\|_{l_{k_{j} k_{j}^{*}}^{\infty} l^{2}}^{4} \cdot\left(L_{1}^{2} L_{2}+L_{1} L_{2}^{2}\right) \sum_{k, k^{\prime}, k_{2}, k_{2}^{\prime}, k_{3}} S_{k+k_{2}-k_{3}, k_{2}, k_{3}} S_{k^{\prime}+k_{2}^{\prime}-k_{3}, k_{2}^{\prime}, k_{3}} .
$$

Then we estimate

$$
\sum_{k, k^{\prime}, k_{3}} \sum_{k_{2}, k_{2}^{\prime}} S_{k+k_{2}-k_{3}, k_{2}, k_{3}} S_{k^{\prime}+k_{2}^{\prime}-k_{3}, k_{2}^{\prime}, k_{3}} \lesssim \sum_{k, k^{\prime}, k_{3}}\left(1+\frac{N_{1}^{2-\alpha}}{\left\langle k-k_{3}\right\rangle}\right)\left(1+\frac{N_{1}^{2-\alpha}}{\left\langle k^{\prime}-k_{3}\right\rangle}\right) \lesssim N_{1}^{2} N_{3} .
$$

Hence

$$
\mathcal{C}_{6} \lesssim N_{1}^{2-4 \alpha} N_{3}\left(L_{1} \wedge L_{2}\right)^{1-4 \nu}\left(L_{2} \vee L_{2}\right)^{2-4 \nu} .
$$

We remark that the estimates for $\mathcal{C}_{3}, \mathcal{C}_{4}, \mathcal{C}_{5}, \mathcal{C}_{6}$ do not use the constraint $\left|k-k^{\prime}\right| \geq L$.

Hence it remains to estimate

$$
\begin{aligned}
& \mathcal{C}_{j}^{\prime}=L N_{1}^{-4 \alpha} \sup _{\substack{k, k^{\prime} \\
\left|k-k^{\prime}\right|<L}} \sum_{\substack{\left(k_{1}, k_{2}, k_{3}\right) \in \Gamma(k) \\
\left(k_{1}^{\prime}, k_{2}^{\prime}, k_{3}\right) \in \Gamma\left(k^{\prime}\right)}} \sum_{\substack{\left(m_{1}, m_{2}, m_{3}\right) \in \Gamma(k) \\
\left(m_{1}^{\prime}, m_{2}^{\prime}, m_{3}\right) \in \Gamma\left(k^{\prime}\right)}} \prod_{j=1}^{2}\left\|h_{k_{j} k_{j}^{*}}\right\|_{l_{k_{j}^{*}}^{2}}\left\|h_{m_{j} m_{j}^{*}}\right\|_{l_{m_{j}^{*}}^{2}}\left\|h_{k_{j}^{\prime} k_{j}^{\prime *}}\right\|_{l_{k_{j}^{\prime *}}^{2}}\left\|h_{m_{j}^{\prime} m_{j}^{\prime *}}\right\|_{l_{m_{j}^{\prime *}}^{2}} \\
& \times S_{k_{1}, k_{2}, k_{3}} S_{k_{1}^{\prime}, k_{2}^{\prime}, k_{3}} S_{m_{1}, m_{2}, m_{3}} S_{m_{1}^{\prime}, m_{2}^{\prime}, m_{3}} \mathbf{1}_{\mathcal{A}_{j}}
\end{aligned}
$$

for $j=1,2$.

For $\mathcal{C}_{1}^{\prime}$, we bound it by (with $k, k^{\prime}$ fixed)

$$
\begin{aligned}
& \mathcal{C}_{1}^{\prime} \lesssim L N_{1}^{-4 \alpha} \prod_{j=1}^{2}\left\|h_{k_{j} k_{j}^{*}}^{N_{j} L_{j}}\right\|_{l_{k_{j}}^{\infty} l_{k_{j}^{*}}^{2 *}}^{4} \cdot \sum_{\substack{k_{2}, k_{2}^{\prime}, k_{3} \\
m_{2}, m_{2}^{\prime}, m_{3}}} S_{k+m_{2}-m_{3}, m_{2}, m_{3}} S_{k^{\prime}+m_{2}^{\prime}-m_{3}, m_{2}^{\prime}, m_{3}}
\end{aligned}
$$

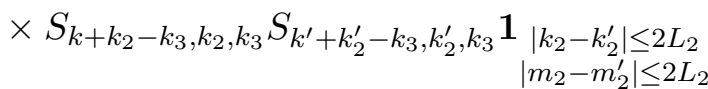

$$
\begin{aligned}
& \lesssim L \cdot L_{2}^{2} N_{1}^{-4 \alpha}\left(L_{1} L_{2}\right)^{-4 \nu} \sum_{k_{2}, m_{2}, k_{3}, m_{3}} S_{k+k_{2}-k_{3}, k_{2}, k_{3}} S_{k+m_{2}-m_{3}, m_{2}, m_{3}} \\
& \lesssim\left(L_{1} \vee L_{2}\right)^{3-4 \nu}\left(L_{1} \wedge L_{2}\right)^{-4 \nu} N_{1}^{-4 \alpha} \sum_{k_{2}, k_{3}, m_{2}} S_{k+k_{2}-k_{3}, k_{2}, k_{3}} \\
& \lesssim\left(L_{1} \vee L_{2}\right)^{3-4 \nu}\left(L_{1} \wedge L_{2}\right)^{-4 \nu} N_{1}^{-4 \alpha} \cdot N_{2}\left(N_{3}+N_{2}^{2-\alpha}\right) \\
& \lesssim\left(L_{1} \vee L_{2}\right)^{3-4 \nu}\left(L_{1} \wedge L_{2}\right)^{-4 \nu}\left(N_{1}^{1-4 \alpha} N_{3}+N_{1}^{2-5 \alpha}\right) \text {. }
\end{aligned}
$$


Next, for fixed $k, k^{\prime}$ (and without loss of generality we assume that $L_{2} \geq L_{1}$ ),

$$
\begin{aligned}
& \mathcal{C}_{2}^{\prime} \lesssim L N_{1}^{-4 \alpha} \prod_{j=1}^{2}\left\|h_{k_{j} k_{j}^{*}}^{N_{j} L_{j}}\right\|_{\substack{l_{k_{j}}^{\infty} l_{k_{j}^{*}} \\
l^{*}}}^{4} \cdot \sum_{\substack{k_{2}, k_{2}^{\prime}, k_{3} \\
m_{2}, m_{2}^{\prime}, m_{3}}} S_{k+m_{2}-m_{3}, m_{2}, m_{3}} S_{k^{\prime}+m_{2}^{\prime}-m_{3}, m_{2}^{\prime}, m_{3}}
\end{aligned}
$$

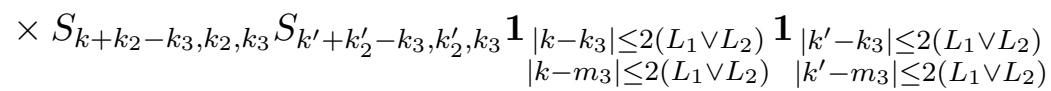

$$
\begin{aligned}
& \lesssim L N_{1}^{-4 \alpha}\left(L_{1} L_{2}\right)^{-4 \nu} \sum_{k_{3}, m_{3}} \mathbf{1}_{\left|k-k_{3}\right| \leq 2\left(L_{1} \vee L_{2}\right)} \mathbf{1}_{\left|k-m_{3}\right| \leq 2\left(L_{1} \vee L_{2}\right)} \mathbf{1}_{\left|k^{\prime}-k_{3}\right| \leq 2\left(L_{1} \vee m_{3} \mid \leq 2\left(L_{1} \vee L_{2}\right)\right.} \\
& \times \sum_{k_{2}} S_{k+k_{2}-k_{3}, k_{2}, k_{3}} \sum_{k_{2}^{\prime}} S_{k^{\prime}+k_{2}^{\prime}-k_{3}, k_{2}^{\prime}, k_{3}} \sum_{m_{2}} S_{k+m_{2}-m_{3}, m_{2}, m_{3}} \sum_{m_{2}^{\prime}} S_{k^{\prime}+m_{2}^{\prime}-m_{3}, m_{2}^{\prime}, m_{3}} \\
& \lesssim\left(L_{1} \vee L_{2}\right)^{1-4 \nu}\left(L_{1} \wedge L_{2}\right)^{-4 \nu} N_{1}^{-8(\alpha-1)},
\end{aligned}
$$

where we use the facts that

$$
\begin{aligned}
& \sum_{k_{2}} S_{k+k_{2}-k_{3}, k_{2}, k_{3}} \mathbf{1}_{\left|k-k_{3}\right| \leq 2\left(L_{1} \vee L_{2}\right)} \lesssim \frac{N_{1}^{2-\alpha}}{\left\langle k-k_{3}\right\rangle}, \\
& \sum_{k_{2}^{\prime}} S_{k^{\prime}+k_{2}^{\prime}-k_{3}, k_{2}, k_{3}} \mathbf{1}_{\left|k^{\prime}-k_{3}\right| \leq 2\left(L_{1} \vee L_{2}\right)} \lesssim \frac{N_{1}^{2-\alpha}}{\left\langle k^{\prime}-k_{3}\right\rangle}
\end{aligned}
$$

and

$$
\begin{aligned}
\sum_{m_{2}} S_{k+m_{2}-m_{3}, m_{2}, m_{3}} \mathbf{1}_{\left|k-m_{3}\right| \leq 2\left(L_{1} \vee L_{2}\right)} \lesssim \frac{N_{1}^{2-\alpha}}{\left\langle k-m_{3}\right\rangle}, \\
\sum_{m_{2}^{\prime}} S_{k^{\prime}+m_{2}^{\prime}-m_{3}, m_{2}, m_{3}} \mathbf{1}_{\left|k^{\prime}-m_{3}\right| \leq 2\left(L_{1} \vee L_{2}\right)} \lesssim \frac{N_{1}^{2-\alpha}}{\left\langle k^{\prime}-m_{3}\right\rangle},
\end{aligned}
$$

since $L_{1} \vee L_{2} \ll N_{1}^{2-\alpha}$. Hence

$$
\mathcal{C}_{2}^{\prime} \lesssim\left(L_{1} \vee L_{2}\right)^{1-4 \nu}\left(L_{1} \wedge L_{2}\right)^{-4 \nu} N_{1}^{-8(\alpha-1)} .
$$

Note that here the treatment is different, compared with $\mathcal{C}_{1}^{\prime}$, due to the different type of pairing. In summary, we have

$$
\begin{aligned}
\mathcal{C}_{1}^{\prime}+\mathcal{C}_{2}^{\prime}+\sum_{j=3}^{6} \mathcal{C}_{j} \lesssim\left(L_{1} \wedge L_{2}\right)^{1-4 \nu}\left(L_{1} \vee L_{2}\right)^{2-4 \nu} N_{3}\left[N_{1}^{2-4 \alpha}+\left(L_{1} L_{2}\right)^{-1} N_{1}^{5-6 \alpha}+N_{1}^{1-4 \alpha}\left(\frac{L_{1} \vee L_{2}}{L_{1} \wedge L_{2}}\right)\right] \\
+\left(L_{1} \wedge L_{2}\right)^{-4 \nu}\left(L_{1} \vee L_{2}\right)^{1-4 \nu} N_{1}^{-8(\alpha-1)}
\end{aligned}
$$

Combining the estimates above and $10.1,(10.2)$, we obtain that:

Proposition 10.3. Assume that $N_{1} \sim N_{2} \gg N_{3}$. Then outside a set of probability $<\mathrm{e}^{-N_{1}^{\theta} R}$, modulo factors $N^{\epsilon}, \epsilon<\kappa^{-0.1}$, we have:

(i) If $a_{1}, a_{2}$ are both of type $(C)$, then

$$
\left|\mathcal{U}_{L_{1}, L_{2}, L_{3}}^{N_{1}, N_{2}, N_{3}}\right| \leq C R N_{1}^{-\frac{3(\alpha-1)}{2}} N_{3}^{-\frac{\alpha-1}{2}}\left(L_{1} L_{2}\right)^{-\nu},
$$

and

$$
\begin{aligned}
\left|\mathcal{U}_{L_{1}, L_{2}, L_{3}}^{N_{1}, N_{2}, N_{3}}\right| & \leq C R N_{1}^{-\frac{3(\alpha-1)}{2}} N_{3}^{-\frac{\alpha-1}{2}}\left(L_{1} L_{2}\right)^{\frac{1}{2}-\nu}\left[N_{3}^{\frac{1}{4}} N_{1}^{-\frac{1}{4}}\left(L_{1} \vee L_{2}\right)^{-\frac{1}{4}}\left(L_{1} \wedge L_{2}\right)^{-\frac{1}{2}}+N_{3}^{\frac{1}{4}} N_{1}^{-\frac{5}{4}+\frac{\alpha}{2}} \frac{\left(L_{1} \vee L_{2}\right)^{\frac{1}{4}}}{\left(L_{1} \wedge L_{2}\right)^{\frac{1}{2}}}\right] \\
& +C R N_{1}^{-\frac{3(\alpha-1)}{2}} N_{3}^{-\frac{\alpha-1}{2}}\left(L_{1} L_{2}\right)^{\frac{1}{2}-\nu} N_{3}^{\frac{1}{4}} N_{1}^{-\left(1-\frac{\alpha}{2}\right)}\left(L_{1} \wedge L_{2}\right)^{-\frac{1}{4}} \\
& +C R\left(L_{1} L_{2}\right)^{-\nu}\left(L_{1} \vee L_{2}\right)^{\frac{1}{4}} N_{1}^{-2(\alpha-1)} N_{3}^{-\frac{\alpha-1}{2}} .
\end{aligned}
$$


(ii) If $a_{1}$ is of type $(C)$ and $a_{2}$ is of type $(G)$ (or $a_{1}$ is of type $(G), a_{2}$ is of type $(C)$ ), then

$$
\left|\mathcal{U}_{L_{1}, L_{2}, L_{3}}^{N_{1}, N_{2}, N_{3}}\right| \leq R N_{1}^{-(\alpha-1)} N_{3}^{-\frac{\alpha-1}{2}} L_{1}^{-\nu},
$$

and

$$
\begin{aligned}
\left|\mathcal{U}_{L_{1}, L_{2}, L_{3}}^{N_{1}, N_{2}, N_{3}}\right| & \leq C R N_{1}^{-\frac{3(\alpha-1)}{2}} N_{3}^{-\frac{\alpha-1}{2}} L_{1}^{-\nu}\left(N_{3}^{\frac{1}{4}} N_{1}^{-\frac{1}{4}} L_{1}^{\frac{1}{4}}+N_{3}^{\frac{1}{4}} N_{1}^{-\frac{5}{4}+\frac{\alpha}{2}} L_{1}^{\frac{3}{4}}\right) \\
& +C R N_{1}^{-\frac{3(\alpha-1)}{2}} N_{3}^{-\frac{\alpha-1}{2}} L_{1}^{-\nu} N_{3}^{\frac{1}{4}} N_{1}^{-\left(1-\frac{\alpha}{2}\right)} L_{1}^{\frac{1}{2}}+C R L_{1}^{\frac{1}{4}-\nu} N_{1}^{-2(\alpha-1)} N_{3}^{-\frac{\alpha-1}{2}} .
\end{aligned}
$$

Note that when $a_{1}$ is of type $(G)$ and $a_{2}$ is of type $(C)$, the estimates above true by switching $L_{1}$ and $L_{2}$.

(iii) If $a_{1}, a_{2}$ are both of type $(G)$, then

$$
\left|\mathcal{U}_{L_{1}, L_{2}, L_{3}}^{N_{1}, N_{2}, N_{3}}\right| \leq C R N_{1}^{\frac{5}{4}-\frac{3 \alpha}{2}} N_{3}^{\frac{3}{4}-\frac{\alpha}{2}}+R N_{1}^{-2(\alpha-1)} N_{3}^{-\frac{\alpha-1}{2}}<C R N_{1}^{-2(\alpha-1)} .
$$

Finally, in whatever the situation, we always have, modulo $N_{1}^{\epsilon}, \epsilon<\kappa^{-0.1}$,

$$
\left|\mathcal{U}_{L_{1}, L_{2}, L_{3}}^{N_{1}, N_{2}, N_{3}}\right| \leq C R \max \left\{N_{1}^{-\left(\frac{3}{2}+2 \nu\right)(\alpha-1)}, N_{1}^{-(\alpha-1)-\nu}, N_{1}^{-\frac{3(\alpha-1)}{2}-\frac{2 \nu}{3}}, N_{1}^{-(1+4 \nu)(\alpha-1)}\right\} .
$$

Proof. We only need to justify the last assertion (10.3). First we assume that $a_{1}, a_{2}$ are both of type (C) and without loss of generality, $L_{1} \geq L_{2}$. Note that, for $A>0, B>0$,

$$
\min \{1, A+B\} \leq \min \{1, A\}+\min \{1, B\} .
$$

Then from the two inequalities of (i), we have a rougher bound

$$
\begin{aligned}
\left|\mathcal{U}_{L_{1}, L_{2}, L_{3}}^{N_{1}, N_{2}, N_{3}}\right| \leq C R N_{1}^{-\frac{3(\alpha-1)}{2}} N_{3}^{-\frac{\alpha-1}{2}}\left(L_{1} L_{2}\right)^{-\nu} \min \left\{1,\left(L_{1} L_{2}\right)^{\frac{1}{2}}\left(\frac{N_{3}}{N_{1}}\right)^{\frac{1}{4}}+L_{1} \frac{N_{3}^{\frac{1}{4}}}{N_{1}^{\frac{5}{4}-\frac{\alpha}{2}}}\right\} \\
+C R N_{1}^{-\frac{3(\alpha-1)}{2}} N_{3}^{-\frac{\alpha-1}{2}}\left(L_{1} L_{2}\right)^{-\nu} \min \left\{1, L_{1}^{\frac{1}{4}} N_{1}^{-\frac{\alpha-1}{2}}\right\} .
\end{aligned}
$$

When $\left(L_{1} L_{2}\right)^{\frac{1}{2}}<N_{1}^{\frac{1}{4}} N_{3}^{-\frac{1}{4}}$, we have

$$
\begin{aligned}
\left|\mathcal{U}_{L_{1}, L_{2}, L_{3}}^{N_{1}, N_{2}, N_{3}}\right| & \leq C R N_{1}^{-\frac{3(\alpha-1)}{2}} N_{3}^{-\frac{\alpha-1}{2}}\left(\left(L_{1} L_{2}\right)^{\frac{1}{2}-\nu} N_{3}^{\frac{1}{4}} N_{1}^{-\frac{1}{4}}+L_{1}^{1-\nu} N_{3}^{\frac{1}{4}} N_{1}^{\frac{\alpha}{2}-\frac{5}{4}}\right) \\
& \leq C R N_{1}^{-\frac{3(\alpha-1)}{2}-\frac{\nu}{2}} N_{3}^{\frac{\nu}{2}-\frac{\alpha-1}{2}}+C R N_{1}^{-\frac{3(\alpha-1)}{2}-\frac{5}{4}+\frac{\alpha}{2}} N_{3}^{-\frac{\alpha-1}{2}+\frac{1}{4}} \cdot\left(N_{1}^{\frac{1}{4}} N_{3}^{-\frac{1}{4}}\right)^{2(1-\nu)} \\
& \leq C R N_{1}^{-\frac{3(\alpha-1)}{2}-\frac{\nu}{2}} N_{3}^{\frac{\nu}{2}-\frac{\alpha-1}{2}}
\end{aligned}
$$

since $L_{1}^{\frac{1}{2}}<N_{1}^{\frac{1}{4}} N_{3}^{-\frac{1}{4}}$ and

$$
N_{1}^{-\frac{3(\alpha-1)}{2}-\frac{5}{4}+\frac{\alpha}{2}} N_{3}^{-\frac{\alpha-1}{2}+\frac{1}{4}} \cdot\left(N_{1}^{\frac{1}{4}} N_{3}^{-\frac{1}{4}}\right)^{2(1-\nu)}=N_{1}^{-\alpha+\frac{3}{4}-\frac{\nu}{2}} N_{3}^{\frac{1}{4}-\frac{\alpha}{2}+\frac{\nu}{2}}<N_{1}^{-\frac{3(\alpha-1)}{2}-\frac{\nu}{2}} N_{3}^{\frac{\nu}{2}-\frac{\alpha-1}{2}} .
$$

When $\left(L_{1} L_{2}\right)^{\frac{1}{2}} \geq N_{1}^{\frac{1}{4}} N_{3}^{-\frac{1}{4}}$, we have simply

$$
N_{1}^{-\frac{3(\alpha-1)}{2}} N_{3}^{-\frac{\alpha-1}{2}}\left(L_{1} L_{2}\right)^{-\nu} \leq C R N_{1}^{-\frac{3(\alpha-1)}{2}-\frac{\nu}{2}} N_{3}^{\frac{\nu}{2}-\frac{\alpha-1}{2}} .
$$

Similarly,

$$
N_{1}^{-\frac{3(\alpha-1)}{2}} N_{3}^{-\frac{\alpha-1}{2}}\left(L_{1} L_{2}\right)^{-\nu} \min \left\{1, L_{1}^{\frac{1}{4}} N_{1}^{-\frac{\alpha-1}{2}}\right\} \leq N_{1}^{-\left(\frac{3}{2}+2 \nu\right)(\alpha-1)} N_{3}^{-\frac{\alpha-1}{2}} .
$$

Therefore, for whatever $L_{1}, L_{2}$ and $N_{3} \ll N_{1}$, we have the bound

$$
\left|\mathcal{U}_{L_{1}, L_{2}, L_{3}}^{N_{1}, N_{2}, N_{3}}\right| \leq C R N_{1}^{-\frac{3(\alpha-1)}{2}-\frac{\nu}{2}} N_{3}^{\frac{\nu}{2}-\frac{\alpha-1}{2}}+C R N_{1}^{-\left(\frac{3}{2}+2 \nu\right)(\alpha-1)} N_{3}^{-\frac{\alpha-1}{2}} .
$$

Note that $\nu>(\alpha-1)$, we have

$$
\left|\mathcal{U}_{L_{1}, L_{2}, L_{3}}^{N_{1}, N_{2}, N_{3}}\right| \leq C R N_{1}^{-2(\alpha-1)}+C R N_{1}^{-\left(\frac{3}{2}+2 \nu\right)(\alpha-1)} .
$$


Next we assume that $a_{1}$ is of type (C) and $a_{2}$ is of type (G), from (ii) we have

$$
\begin{aligned}
&\left|\mathcal{U}_{L_{1}, L_{2}, L_{3}}^{N_{1}, N_{2}, N_{3}}\right| \leq C R N_{1}^{-(\alpha-1)} N_{3}^{-\frac{\alpha-1}{2}} L_{1}^{-\nu} \min \left\{1, L_{1}^{\frac{1}{4}} N_{3}^{\frac{1}{4}} N_{1}^{-\frac{1}{4}-\frac{\alpha-1}{2}}\right\} \\
&+C R N_{1}^{-(\alpha-1)} N_{3}^{-\frac{\alpha-1}{2}} L_{1}^{-\nu} \min \left\{1, N_{3}^{\frac{1}{4}} N_{1}^{-\frac{5}{4}+\frac{\alpha}{2}-\frac{\alpha-1}{2}} L_{1}^{\frac{3}{4}}\right\} \\
& \\
&+C R N_{1}^{-(\alpha-1)} N_{3}^{-\frac{\alpha-1}{2}} L_{1}^{-\nu} \min \left\{1, L_{1}^{\frac{1}{2}} N_{3}^{\frac{1}{4}} N_{1}^{-\left(1-\frac{\alpha}{2}\right)-\frac{\alpha-1}{2}}\right\} \\
&+C R N_{1}^{-(\alpha-1)} N_{3}^{-\frac{\alpha-1}{2}} L_{1}^{-\nu} \min \left\{1, L_{1}^{\frac{1}{4}} N_{1}^{-(\alpha-1)}\right\} .
\end{aligned}
$$

Note that

$N_{1}^{-(\alpha-1)} N_{3}^{-\frac{\alpha-1}{2}} L_{1}^{-\nu} \min \left\{1, L_{1}^{\frac{1}{4}} N_{3}^{\frac{1}{4}} N_{1}^{-\frac{1}{4}-\frac{\alpha-1}{2}}\right\} \leq N_{1}^{-(\alpha-1)-\nu(1+2(\alpha-1))} N_{3}^{\nu-\frac{\alpha-1}{2}} \leq N_{1}^{-\left(\frac{3}{2}+2 \nu\right)(\alpha-1)}$,

since $\nu>\alpha-1$ and thus $N_{3}^{\frac{\nu}{2}-\frac{\alpha-1}{2}}<N_{1}^{\frac{\nu}{2}-\frac{\alpha-1}{2}}$. Next,

$$
\begin{aligned}
N_{1}^{-(\alpha-1)} N_{3}^{-\frac{\alpha-1}{2}} L_{1}^{-\nu} \min \left\{1, N_{3}^{\frac{1}{4}} N_{1}^{-\frac{5}{4}+\frac{\alpha}{2}-\frac{\alpha-1}{2}} L_{1}^{\frac{3}{4}}\right\} & \leq N_{1}^{-(\alpha-1)-\nu} N_{3}^{\frac{\nu}{3}-\frac{\alpha-1}{2}} \\
& \leq \max \left\{N_{1}^{-(\alpha-1)-\nu}, N_{1}^{-\frac{3(\alpha-1)}{2}-\frac{2 \nu}{3}}\right\} .
\end{aligned}
$$

For the third term, we have

$$
\begin{aligned}
N_{1}^{-(\alpha-1)} N_{3}^{-\frac{\alpha-1}{2}} L_{1}^{-\nu} \min \left\{1, L_{1}^{\frac{1}{2}} N_{3}^{\frac{1}{4}} N_{1}^{-\left(1-\frac{\alpha}{2}\right)-\frac{\alpha-1}{2}}\right\} & \leq N_{1}^{-(\alpha-1)-\nu} N_{3}^{\frac{\nu}{2}-\frac{\alpha-1}{2}} \\
& \leq N_{1}^{-\frac{3}{2}(\alpha-1)-\frac{\nu}{2}}<N_{1}^{-\left(\frac{3}{2}+2 \nu\right)(\alpha-1)},
\end{aligned}
$$

since $\alpha<\frac{5}{4}$. Finally,

$$
N_{1}^{-(\alpha-1)} N_{3}^{-\frac{\alpha-1}{2}} L_{1}^{-\nu} \min \left\{1, L_{1}^{\frac{1}{4}} N_{1}^{-(\alpha-1)}\right\} \leq N_{1}^{-(1+4 \nu)(\alpha-1)} N_{3}^{-\frac{\alpha-1}{2}} \leq N_{1}^{-(1+4 \nu)(\alpha-1)} .
$$

Therefore, we have

$$
\left|\mathcal{U}_{L_{1}, L_{2}, L_{3}}^{N_{1}, N_{2}, N_{3}}\right| \leq C R \max \left\{N_{1}^{-\left(\frac{3}{2}+2 \nu\right)(\alpha-1)}, N_{1}^{-(\alpha-1)-\frac{3}{4} \nu}, N_{1}^{-(1+4 \nu)(\alpha-1)}\right\} .
$$

This completes the proof of Proposition 10.3 .

10.2. The case $N_{1} \sim N_{3} \gg N_{2}$. For the high-low-high interactions, first we deal with

-Case A-4: At least one of $a_{1}, a_{3}$ is of type (D)

We execute Algorithm 4. By inserting the indicator $\mathbf{1}_{k_{1} \cdot k_{3} \geq 0}$ and $\mathbf{1}_{k_{1} \cdot k_{2}<0}$, we have

$$
\begin{aligned}
\left|\mathcal{U}_{L_{1}, L_{2}, L_{3}}^{N_{1}, N_{2}, N_{3}}\right|^{2} \lesssim\left(\sum_{|k| \leq N_{1}} \sum_{\left(k_{1}, k_{2}, k_{3}\right) \in \Gamma(k)}\left|a_{1}\left(k_{1}\right)\right|^{2}\left|a_{2}\left(k_{2}\right)\right|^{2} \mathbf{1}_{k_{1} k_{3} \geq 0}\right) \cdot\left(\sup _{k} \sum_{\left(k_{1}, k_{2}, k_{3}\right) \in \Gamma(k)}\left|a_{3}\left(k_{3}\right)\right|^{2}\right) \\
+\left(\sum_{|k| \leq N_{1}} \sum_{\left(k_{1}, k_{2}, k_{3}\right) \in \Gamma(k)}\left|a_{1}\left(k_{1}\right)\right|^{2}\left|a_{2}\left(k_{2}\right)\right|^{2} \mathbf{1}_{k_{1} k_{3}<0}\right) \cdot\left(\sup _{k} \sum_{\left(k_{1}, k_{2}, k_{3}\right) \in \Gamma(k)}\left|a_{3}\left(k_{3}\right)\right|^{2}\right) .
\end{aligned}
$$

When $k_{1} k_{3} \geq 0$, for fixed $k_{1}, k_{2}$, we have

$$
\left|\partial_{k_{3}} \Phi_{k_{1}, k_{2}, k_{3}}\right|=\left.\alpha\left|\operatorname{sign}\left(k_{3}\right)\right| k_{3}\right|^{\alpha-1}-\operatorname{sign}\left(k_{1}-k_{2}+k_{3}\right)\left|k_{1}-k_{2}+k_{3}\right|^{\alpha-1} \mid \gtrsim N_{1}^{\alpha-1},
$$

hence

$$
\sum_{|k| \leq N_{1}} \sum_{\left(k_{1}, k_{2}, k_{3}\right) \in \Gamma(\bar{k})}\left|a_{1}\left(k_{1}\right)\right|^{2}\left|a_{2}\left(k_{2}\right)\right|^{2} \mathbf{1}_{k_{1} k_{3} \geq 0} \lesssim\left\|a_{1}\right\|_{l^{2}}^{2}\left\|a_{2}\right\|_{l^{2}}^{2}
$$

Since $\left|k+k_{2}-k_{3}\right|=\left|k_{1}\right| \gg\left|k_{2}\right|$, for fixed $k, k_{3}$,

$\left|\partial_{k_{2}} \Phi\left(k+k_{2}-k_{3}, k_{2}, k_{3}\right)\right|=\alpha\left|\operatorname{sign}\left(k+k_{2}-k_{3}\right)\right| k+k_{2}-\left.k_{3}\right|^{\alpha-1}-\operatorname{sign}\left(k_{2}\right)\left|k_{2}\right|^{\alpha-1} \mid \sim N_{1}^{\alpha-1}$.

Thus

$\left(\sum_{|k| \leq N_{1}} \sum_{\left(k_{1}, k_{2}, k_{3}\right) \in \Gamma(\bar{k})}\left|a_{1}\left(k_{1}\right)\right|^{2}\left|a_{2}\left(k_{2}\right)\right|^{2} \mathbf{1}_{k_{1} k_{3} \geq 0}\right) \cdot\left(\sup _{k} \sum_{\left(k_{1}, k_{2}, k_{3}\right) \in \Gamma(\bar{k})}\left|a_{3}\left(k_{3}\right)\right|^{2} \mathbf{1}_{k_{1} \cdot k_{3} \geq 0}\right) \lesssim \prod_{j=1}^{3}\left\|a_{j}\right\|_{l^{2}}^{2}$. 
When $k_{1} k_{3}<0$, if $\operatorname{sign}\left(k_{3}\right) \neq \operatorname{sign}\left(k_{1}-k_{2}+k_{3}\right),\left|\partial_{k_{3}} \Phi_{k_{1}, k_{2}, k_{3}}\right| \gtrsim N_{1}^{\alpha-1}$. If $\operatorname{sign}\left(k_{3}\right)=\operatorname{sign}\left(k_{3}-\right.$ $\left.\left(-k_{1}+k_{2}\right)\right)$, we must have $\left|k_{3}\right|>\left|k_{1}-k_{2}\right|$. Without loss of generality, we may assume that $k_{3}>0$, hence

$$
\left|\partial_{k_{3}} \Phi_{k_{1}, k_{2}, k_{3}}\right|=\left.\alpha(\alpha-1)\left|\int_{k_{3}-k_{2}+k_{1}}^{k_{3}}\right| x\right|^{\alpha-2} d x \mid \gtrsim \frac{\left|k_{1}-k_{2}\right|}{\left|k_{3}\right|^{2-\alpha}} \sim N_{1}^{\alpha-1} .
$$

Therefore,

$$
\left(\sum_{|k| \leq N_{1}} \sum_{\left(k_{1}, k_{2}, k_{3}\right) \in \Gamma(\bar{k})}\left|a_{1}\left(k_{1}\right)\right|^{2}\left|a_{2}\left(k_{2}\right)\right|^{2} \mathbf{1}_{k_{1} k_{3}<0}\right) \cdot\left(\sup _{k} \sum_{\left(k_{1}, k_{2}, k_{3}\right) \in \Gamma(\bar{k})}\left|a_{3}\left(k_{3}\right)\right|^{2} \mathbf{1}_{k_{1} \cdot k_{3} \geq 0}\right) \lesssim \prod_{j=1}^{3}\left\|a_{j}\right\|_{l^{2}}^{2} .
$$

Therefore, we have proved:

Proposition 10.4. Assume that $N_{1} \sim N_{3} \gg N_{2}$ and at least one of $a_{1}, a_{2}$ is of type (D), then

$$
\mathcal{U}_{L_{1}, L_{2}, L_{3}}^{N_{1}, N_{2}, N_{3}} \lesssim \prod_{j=1}^{3}\left\|a_{j}\right\|_{l^{2}} \lesssim N_{1}^{-\frac{\alpha-1}{2}-s} N_{3}^{-\frac{\alpha-1}{2}}
$$

- $a_{1}, a_{3}$ are both of type $(\mathbf{G})$ or $(\mathbf{C})$

This situation is similar to Case A-3 and we can obtain the same upper bound. For this reason, we omit the details. Finally, we remark that from the choice of parameters $\nu, s$ in Remark 3.1, under the constraint $\alpha>\alpha_{0}$, there exists $\sigma>0$, sufficiently small, such that (1) of Proposition 3.7 holds in the situation $N_{(1)} \sim N_{(2)} \gg N_{(3)}$.

\section{High-LOW-LOW INTERACTIONS}

In this section, we finish the proof of Proposition 3.7 by showing (2), (4) and the regime $N_{(1)} \gg N_{(2)} \gtrsim N_{(1)}^{1-\delta}$ or $N_{(1)} \gg N_{(2)}$ and $N_{2} \sim N_{(1)}$ for (1).

-Case B-1: $N_{1} \gg N_{2}, N_{3}, a_{1}$ is of type (D) and $N_{(2)} \gtrsim N_{(1)}^{1-\delta}$

In this case, we execute Algorithm 4. By Cauchy-Schwartz,

$$
\left|\mathcal{U}_{L_{1}, L_{2}, L_{3}}^{N_{1}, N_{2}, N_{3}}\right|^{2} \leq\left(\sum_{k_{1}, k_{2}, k_{3}} S_{k_{1}, k_{2}, k_{3}}\left|a_{1}\left(k_{1}\right)\right|^{2}\left|a_{2}\left(k_{2}\right)\right|^{2}\right) \cdot \sup _{k}\left(\sum_{k_{2}, k_{3}}\left|a_{3}\left(k_{3}\right)\right|^{2} S_{k+k_{2}-k_{3}, k_{2}, k_{3}}\right) .
$$

Since $N_{1} \gg N_{2}, N_{3}$,

$$
\left|\partial_{k_{2}} \Phi\left(k+k_{2}-k_{3}, k_{2}, k_{3}\right)\right| \gtrsim N_{1}^{\alpha-1}, \quad\left|\partial_{k_{3}} \Phi_{k_{1}, k_{2}, k_{3}}\right| \gtrsim N_{1}^{\alpha-1},
$$

we have

$$
\sum_{k_{2}} S_{k+k_{2}-k_{3}, k_{2}, k_{3}} \lesssim 1, \quad \sum_{k_{3}} S_{k_{1}, k_{2}, k_{3}} \lesssim 1
$$

hence

$$
\left|\mathcal{U}_{L_{1}, L_{2}, L_{3}}^{N_{1}, N_{2}, N_{3}}\right| \lesssim\left\|a_{1}\right\|_{l^{2}}\left\|a_{2}\right\|_{l^{2}}\left\|a_{3}\right\|_{l^{2}} .
$$

Therefore, we have proved:

Proposition 11.1. Assume that $N_{1} \gg N_{2}, N_{3}, N_{(2)} \gtrsim N_{(1)}^{1-\delta}$ and $a_{1}$ is of type (D), then

$$
\left|\mathcal{U}_{L_{1}, L_{2}, L_{3}}^{N_{1}, N_{2}, N_{3}}\right| \lesssim \prod_{j=1}^{3}\left\|a_{j}\right\|_{l^{2}} \lesssim N_{(1)}^{-s-\frac{\alpha-1}{2}(1-\delta)}
$$

where the factor $N^{-\frac{\alpha-1}{2}}$ comes from the worst case when $a_{(2)}$ is of type $(G)$.

-Case B-2: $N_{1} \gg N_{2}, N_{3}, a_{1}$ is of type (G) or (C), $a_{(2)}$ is of type (D) and $N_{(2)} \gtrsim N_{(1)}^{1-\delta}$

The estimate in this case is the same as Case B-1, and we summarize as follows: 
Proposition 11.2. Assume that $N_{1} \gg N_{2}, N_{3}, N_{(2)} \gtrsim N_{(1)}^{1-\delta}$ and $a_{1}$ is of type $(G)$ or $(C)$, then

$$
\left|\mathcal{U}_{L_{1}, L_{2}, L_{3}}^{N_{1}, N_{2}, N_{3}}\right| \lesssim \prod_{j=1}^{3}\left\|a_{j}\right\|_{l^{2}} \lesssim N_{(1)}^{-(1-\delta) s-\frac{\alpha-1}{2}},
$$

where the factor $N^{-\frac{\alpha-1}{2}}$ comes from the worst case when $a_{1}$ is of type $(G)$.

-Case B-3: $N_{1} \gg N_{2}, N_{3}, a_{(1)}, a_{(2)}$ are both of type $(\mathbf{G})$ or $(\mathbf{C})$ and $N_{(2)} \gtrsim N_{(1)}^{1-\delta}$

If $a_{(1)}, a_{(2)}$ are both of type $(\mathrm{C})$, then we can apply the same argument as for Case B-1 to obtain that

$$
\left|\mathcal{U}_{L_{1}, L_{2}, L_{3}}^{N_{1}, N_{2}, N_{3}}\right| \lesssim \prod_{j=1}^{3}\left\|a_{j}\right\|_{l^{2}} \lesssim N_{(1)}^{-2(\alpha-1)(1-\delta)},
$$

which is conclusive. Therefore we may assume that at least one of $a_{(1)}, a_{(2)}$ is of type $(\mathrm{G})$. If $a_{(1)}$ is of type (C) and $L_{(1)} \gtrsim N_{(2)}\left(\gtrsim N_{(1)}^{1-\delta}\right)$, we have the deterministic bound

$$
\left|\mathcal{U}_{L_{1}, L_{2}, L_{3}}^{N_{1}, N_{2}, N_{3}}\right| \lesssim \prod_{j=1}^{3}\left\|a_{j}\right\|_{l^{2}} \lesssim N_{(1)}^{-(\alpha-1)} L_{(1)}^{-\nu} N_{(2)}^{-\frac{\alpha-1}{2}} \lesssim N_{(1)}^{-\frac{3(\alpha-1)(1-\delta)}{2}-\nu}
$$

which is also conclusive. Now we assume that $L_{(1)} \ll N_{(2)}$, then this situation is essentially the same as Case A-3. Revisiting all the analysis for Case A-3 and Case A-4, the only difference here is that we should replace $N_{(2)} \sim N_{(1)}$ by $N_{(2)} \sim N_{(1)}^{1-\delta}$. All the outputs of the summations like $\sum_{k_{2}} S_{k_{1}, k_{2}, k_{3}}, \sum_{k_{3}} S_{k_{1}, k_{2}, k_{3}}, \sum_{k_{1}} S_{k_{1}, k_{2}, k_{3}}$ and $\sum_{k} S_{k+k_{2}-k_{3}, k_{2}, k_{3}}$ are smaller than the case $N_{(1)} \sim N_{(2)} \gg N_{(3)}$, up to a loss of small power $N_{(1)}^{\delta}$. We omit the details.

-Case B-4: $N_{2} \gg N_{1}, N_{3}, a_{2}$ is of type (G) or (C)

We execute Algorithm 3. By Cauchy-Schwartz,

$$
\left|\mathcal{U}_{L_{1}, L_{2}, L_{3}}^{N_{1}, N_{2}, N_{3}}\right|^{2} \leq\left(\sum_{k_{1}, k_{2}, k_{3}}\left|a_{1}\left(k_{1}\right)\right|^{2}\left|a_{2}\left(k_{2}\right)\right|^{2} S_{k_{1}, k_{2}, k_{3}}\right) \cdot \sup _{k}\left(\sum_{k_{1}, k_{3}}\left|a_{3}\left(k_{3}\right)\right|^{2} S_{k_{1}, k_{1}+k_{3}-k, k_{3}}\right) .
$$

Note that for fixed $k, k_{2},\left|\partial_{k_{1}} \Phi\left(k_{1}, k_{1}+k_{3}-k, k_{3}\right)\right| \gtrsim N_{2}^{\alpha-1}$ since $N_{1} \ll N_{2}$, we have

$$
\sum_{k_{1}} S_{k_{1}, k_{1}+k_{3}-k, k_{3}} \lesssim 1
$$

For fixed $k_{1}, k_{3}$, by Lemma 2.9 ,

$$
\sum_{k_{2}} S_{k_{1}, k_{2}, k_{3}} \lesssim N_{2}^{1-\frac{\alpha}{2}}
$$

Thus we have

$$
\left|\mathcal{U}_{L_{1}, L_{2}, L_{3}}^{N_{1}, N_{2}, N_{3}}\right| \lesssim N_{2}^{\frac{1}{2}-\frac{\alpha}{4}}\left\|a_{1}\right\|_{l^{2}}\left\|a_{2}\right\|_{l^{\infty}}\left\|a_{3}\right\|_{l^{2}} \leq C R N_{1}^{-\frac{\alpha-1}{2}} N_{3}^{-\frac{\alpha-1}{2}} N_{2}^{\frac{1}{2}-\frac{3 \alpha}{4}} \leq C R N_{(1)}^{-\frac{\alpha}{4}-\frac{\alpha-1}{2}} .
$$

We have proved:

Proposition 11.3. Assume that $N_{2} \gg N_{1}, N_{3}$ and $a_{2}$ is of type $(G)$ or $(C)$. We have

$$
\left|\mathcal{U}_{L_{1}, L_{2}, L_{3}}^{N_{1}, N_{2}, N_{3}}\right| \leq C R N_{(1)}^{-\frac{\alpha}{4}-\frac{\alpha-1}{2}} .
$$

-Case B-5: Projective terms $\Pi_{N_{1}}^{\perp} \mathcal{I} \mathcal{N}_{3}\left(v_{1}, v_{2}, v_{3}\right)$ and $a_{1}$ is of type (G) or (C)

This time we denote slight differently by

$$
\widetilde{\mathcal{U}}_{L_{1}, L_{2}, L_{3}}^{N_{1}, N_{2}, N_{3}}:=\left(\sum_{|k|>N_{1}}\left|\sum_{\substack{\left(k_{1}, k_{2}, k_{3}\right) \in \Gamma(k) \\\left|k_{j}\right| \lesssim N_{j}, j=1,2,3}} a_{1}\left(k_{1}\right) \bar{a}_{2}\left(k_{2}\right) a_{3}\left(k_{3}\right)\right|^{2}\right)^{\frac{1}{2}} .
$$


The key point here is that the range of $k_{1}$ in the summation satisfies

$$
N_{1}-\left(N_{2} \vee N_{3}\right) \leq N_{1}-\left|k_{2}-k_{3}\right|<\left|k_{1}\right|<N_{1}+\left|k_{2}-k_{3}\right|<N_{1}+\left(N_{2} \vee N_{3}\right),
$$

hence for fixed $k_{2}, k_{3}$, the range of $k$ (or $k_{1}$ ) is at most $2\left|k_{2}-k_{3}\right|$. We have the following improved counting bound (again we ignore small powers of $N_{1}$ in the definition of $S_{k_{1}, k_{2}, k_{3}}$ ):

$$
\sum_{|k|>N_{1}} S_{k+k_{2}-k_{3}, k_{2}, k_{3}} \lesssim \min \left\{\left\langle k_{2}-k_{3}\right\rangle,\left(1+\frac{N_{1}^{2-\alpha}}{\left\langle k_{2}-k_{3}\right\rangle}\right)\right\} .
$$

Now let us first execute Algorithm 1. Without loss of generality, we assume that $N_{3} \leq$ $N_{2}$. By Cauchy-Schwartz, we have

$$
\begin{aligned}
\left|\widetilde{\mathcal{U}}_{L_{1}, L_{2}, L_{3}}^{N_{1}, N_{2}, N_{3}}\right|^{2} & \leq \sum_{\substack{k, k_{2}, k_{3} \\
|k|>N_{1}}}\left|a_{1}\left(k+k_{2}-k_{3}\right)\right|^{2}\left|a_{2}\left(k_{2}\right)\right|^{2} S_{k+k_{2}-k_{3}, k_{2}, k_{3}} \cdot \sup _{|k|>N_{1}} \sum_{k_{2}, k_{3}}\left|a_{3}\left(k_{3}\right)\right|^{2} S_{k+k_{2}-k_{3}, k_{2}, k_{3}} \\
& \lesssim\left\|a_{1}\right\|_{l_{k_{1}}}^{2}\left\|a_{3}\right\|_{l_{k_{3}}^{2}}^{2} \sum_{k_{2}, k_{3}}\left|a_{2}\left(k_{2}\right)\right|^{2} \min \left\{\left\langle k_{2}-k_{3}\right\rangle, 1+\frac{N_{1}^{2-\alpha}}{\left\langle k_{2}-k_{3}\right\rangle}\right\},
\end{aligned}
$$

where we used (11.4) and the fact that $\sum_{k_{2}} S_{k+k_{2}-k_{3}, k_{2}, k_{3}} \lesssim 1$ in the last step. Note that $\left\langle k_{2}-k_{3}\right\rangle<1+\frac{N_{1}^{2-\alpha}}{\left\langle k_{2}-k_{3}\right\rangle}$ only if $\left\langle k_{2}-k_{3}\right\rangle \lesssim N_{1}^{1-\frac{\alpha}{2}}$. Hence if $N_{2} \ll N_{1}^{1-\frac{\alpha}{2}}$, we can bound $\left|\widetilde{\mathcal{U}}_{L_{1}, L_{2}, L_{3}}^{N_{1}, N_{2}, N_{3}}\right|^{2}$ by $N_{2} N_{3}\left\|a_{1}\right\|_{l^{\infty}}^{2}\left\|a_{2}\right\|_{l^{2}}^{2}\left\|a_{3}\right\|_{l^{2}}^{2}$. When $N_{2} \gtrsim N_{1}^{1-\frac{\alpha}{2}}$, we can split the sum of $k_{3}$ into $\left\langle k_{3}-k_{2}\right\rangle \leq N_{1}^{1-\frac{\alpha}{2}}$ and $\left\langle k_{3}-k_{2}\right\rangle>N_{1}^{1-\frac{\alpha}{2}}$.The sum (over $k_{2}, k_{3}$ ) for the former case can be bounded by

$$
\left\|a_{2}\right\|_{l^{2}}^{2} \min \left\{N_{3} N_{1}^{1-\frac{\alpha}{2}}, N_{1}^{2-\alpha} \log \left(N_{2}\right)\right\}
$$

while the sum for the later case can be bounded by

$$
\left\|a_{2}\right\|_{l^{2}}^{2}\left(N_{3}+\min \left\{N_{1}^{2-\alpha} \log \left(N_{3}\right), N_{3} N_{1}^{1-\frac{\alpha}{2}}\right\}\right) .
$$

Therefore, we have

$$
\left|\widetilde{\mathcal{U}}_{L_{1}, L_{2}, L_{3}}^{N_{1}, N_{2}, N_{3}}\right|^{2} \lesssim\left\|a_{1}\right\|_{l^{\infty}}^{2}\left\|a_{2}\right\|_{l^{2}}^{2}\left\|a_{3}\right\|_{l^{2}}^{2}\left(N_{3}+\min \left\{N_{1}^{2-\alpha}+N_{3} N_{1}^{1-\frac{\alpha}{2}}\right\}\right) \log \left(N_{2}\right) .
$$

Lemma 11.4. Assume that $N_{2}, N_{3}<N_{1}^{1-\delta}$. Then modulo small powers of $N_{1}$, we have:

(1) $\left|\widetilde{\mathcal{U}}_{L_{1}, L_{2}, L_{3}}^{N_{1}, N_{2}, N_{3}}\right| \lesssim\left(N_{2} N_{3}\right)^{\frac{1}{2}}\left\|a_{1}\right\|_{l^{\infty}}\left\|a_{2}\right\|_{l^{2}}\left\|a_{3}\right\|_{l^{2}}, \quad$ if $N_{2} \vee N_{3} \ll N_{1}^{1-\frac{\alpha}{2}}$;

(2) $\left|\widetilde{\mathcal{U}}_{L_{1}, L_{2}, L_{3}}^{N_{1}, N_{2}, N_{3}}\right| \lesssim\left[\left(N_{2} \wedge N_{3}\right)^{\frac{1}{2}}+N_{1}^{\frac{1}{2}-\frac{\alpha}{4}} \min \left\{\left(N_{2} \wedge N_{3}\right)^{\frac{1}{2}}, N_{1}^{\frac{1}{2}-\frac{\alpha}{4}}\right\}\right]\left\|a_{1}\right\| l_{l^{\infty}}\left\|a_{2}\right\|_{l^{2}}\left\|a_{3}\right\|_{l^{2}}$,

$$
\text { if } N_{2} \vee N_{3} \gtrsim N_{1}^{1-\frac{\alpha}{2}} \text {. }
$$

Consequently, we have:

Corollary 11.5. Assume that $N_{2}, N_{3}<N_{1}^{1-\delta}$. Then modulo small powers of $N_{1}$ :

- If $a_{2}, a_{3}$ are both of type (D), then

$$
\left|\widetilde{\mathcal{U}}_{L_{1}, L_{2}, L_{3}}^{N_{1}, N_{2}, N_{3}}\right| \lesssim N_{1}^{-\frac{\alpha-1}{2}-\frac{\alpha^{2}}{8}}
$$

- If $L_{1} \gtrsim N_{2} \wedge N_{3}$, then

$$
\left|\widetilde{\mathcal{U}}_{L_{1}, L_{2}, L_{3}}^{N_{1}, N_{2}, N_{3}}\right| \lesssim N_{1}^{-(\alpha-1+\nu)\left(1-\frac{\alpha}{2}\right)-(\alpha-1)}+N_{1}^{-\frac{3(\alpha-1)}{2}-\nu} .
$$

In particular, when $\alpha>\alpha_{0}$, for sufficiently small free parameter $\sigma>0$, we have

$$
\left|\widetilde{\mathcal{U}}_{L_{1}, L_{2}, L_{3}}^{N_{1}, N_{2}, N_{3}}\right| \lesssim N_{1}^{-s-\delta_{0}}
$$


Proof. Assume that $a_{2}, a_{3}$ are both of type (D). From Lemma 11.4, when $N_{2} \vee N_{3} \ll N_{1}^{1-\frac{\alpha}{2}}$, we have

$$
\left|\widetilde{\mathcal{U}}_{L_{1}, L_{2}, L_{3}}^{N_{1}, N_{2}, N_{3}}\right| \lesssim\left(N_{2} N_{3}\right)^{\frac{1}{2}-s} N_{1}^{-\frac{\alpha}{2}} \leq N_{1}^{-\frac{\alpha}{2}}\left(N_{2} \vee N_{3}\right)^{1-2 s} \ll N_{1}^{-\frac{\alpha^{2}}{4}},
$$

since $s=\frac{1}{2}-\frac{\alpha}{4}+\sigma>\frac{1}{2}-\frac{\alpha}{4}$. Now we assume without loss of generality that $N_{3} \leq N_{2}, N_{2} \gtrsim$ $N_{1}^{1-\frac{\alpha}{2}}$. If $N_{3} \leq N_{1}^{1-\frac{\alpha}{2}}$, we have

$$
\begin{aligned}
\left|\widetilde{\mathcal{U}}_{L_{1}, L_{2}, L_{3}}^{N_{1}, N_{2}, N_{3}}\right| & \lesssim N_{3}^{\frac{1}{2}} N_{1}^{\frac{1}{2}-\frac{\alpha}{4}} \cdot N_{1}^{-\frac{\alpha}{2}} N_{2}^{-s} N_{3}^{-s} \leq N_{3}^{\frac{1}{2}-s} N_{1}^{\frac{1}{2}\left(1-\frac{\alpha}{2}\right)-s\left(1-\frac{\alpha}{2}\right)} N_{1}^{-\frac{\alpha}{2}} \\
& \leq N_{1}^{\left(\frac{1}{2}-s\right)\left(1-\frac{\alpha}{2}\right)+\left(\frac{1}{2}-s\right)\left(1-\frac{\alpha}{2}\right)} N_{1}^{-\frac{\alpha}{2}} \leq N_{1}^{-\frac{\alpha^{2}}{4}+\delta_{0}} N_{2}^{-\delta_{0}} .
\end{aligned}
$$

When $N_{3}>N_{1}^{1-\frac{\alpha}{2}}$, we have

$$
\begin{aligned}
\left|\widetilde{\mathcal{U}}_{L_{1}, L_{2}, L_{3}}^{N_{1}, N_{2}, N_{3}}\right| & \lesssim\left(N_{3}^{\frac{1}{2}}+N_{1}^{1-\frac{\alpha}{2}}\right) \cdot N_{1}^{-\frac{\alpha}{2}} N_{2}^{-s} N_{3}^{-s} \leq\left(N_{2}^{\frac{1}{2}-2 s}+N_{1}^{1-\frac{\alpha}{2}} N_{2}^{-s}\right) N_{1}^{-\frac{\alpha}{2}} \\
& \leq\left(N_{1}^{-\frac{\alpha-1}{2}-2 s}+N_{1}^{-\frac{\alpha-1}{2}-\frac{\alpha^{2}}{8}}\right) .
\end{aligned}
$$

Note that when $\alpha>\alpha_{0}, \frac{\alpha-1}{2}+\frac{\alpha^{2}}{8}>s$ for very small free numerical parameter $\sigma>0$, thus we obtain the first inequality.

Next we assume that $L_{1} \gtrsim N_{2} \wedge N_{3}$, from $\left\|a_{1}\right\|_{l^{\infty}} \lesssim N_{1}^{-\frac{\alpha}{2}} L_{1}^{-\nu} \lesssim N_{1}^{-\frac{\alpha}{2}}\left(N_{2} \wedge N_{3}\right)^{-\nu}$, we have, if $N_{2} \vee N_{3} \ll N_{1}^{1-\frac{\alpha}{2}}$,

$$
\left|\widetilde{\mathcal{U}}_{L_{1}, L_{2}, L_{3}}^{N_{1}, N_{2}, N_{3}}\right| \lesssim\left(N_{2} N_{3}\right)^{\frac{1}{2}} N_{1}^{-\frac{\alpha}{2}}\left(N_{2} \wedge N_{3}\right)^{-\nu}\left\|a_{2}\right\|_{l^{2}}\left\|a_{3}\right\|_{l^{2}}
$$

In the worst case, $\left\|a_{j}\right\|_{l^{2}} \lesssim N_{j}^{-\frac{\alpha-1}{2}}$ for $j=2,3$, we have

$$
\left|\widetilde{\mathcal{U}}_{L_{1}, L_{2}, L_{3}}^{N_{1}, N_{2}, N_{3}}\right| \lesssim\left(N_{2} \vee N_{3}\right)^{2-\alpha-\nu} N_{1}^{-\frac{\alpha}{2}}<N_{1}^{2-\frac{3 \alpha}{2}-(\alpha+\nu)\left(1-\frac{\alpha}{2}\right)},
$$

since $2-\alpha-\nu>0$. Now we assume that $N_{2} \gtrsim N_{1}^{1-\frac{\alpha}{2}}$ and $N_{3} \leq N_{2}$. If $N_{3} \leq N_{1}^{1-\frac{\alpha}{2}}$, we have

$$
\begin{aligned}
\left|\widetilde{\mathcal{U}}_{L_{1}, L_{2}, L_{3}}^{N_{1}, N_{2}, N_{3}}\right| & \lesssim N_{3}^{\frac{1}{2}} N_{1}^{\frac{1}{2}\left(1-\frac{\alpha}{2}\right)} \cdot N_{1}^{-\frac{\alpha}{2}} N_{2}^{-\frac{\alpha-1}{2}} N_{3}^{-\nu-\frac{\alpha-1}{2}} \leq N_{1}^{\left(1-\frac{\alpha}{2}-\nu-\frac{\alpha-1}{2}\right)\left(1-\frac{\alpha}{2}\right)+\frac{1}{2}\left(1-\frac{\alpha}{2}\right)} \cdot N_{1}^{-\frac{\alpha}{2}} \\
& \leq N_{1}^{-(\alpha+\nu)\left(1-\frac{\alpha}{2}\right)+2-\frac{3 \alpha}{2}}=N_{1}^{-(\alpha-1+\nu)\left(1-\frac{\alpha}{2}\right)-(\alpha-1)},
\end{aligned}
$$

since $1-\frac{\alpha}{2}-\nu>0$. When $N_{3}>N_{1}^{1-\frac{\alpha}{2}}$, we have

$$
\begin{aligned}
\left|\widetilde{\mathcal{U}}_{L_{1}, L_{2}, L_{3}}^{N_{1}, N_{2}, N_{3}}\right| & \lesssim\left(N_{3}^{\frac{1}{2}}+N_{1}^{1-\frac{\alpha}{2}}\right) \cdot N_{1}^{-\frac{\alpha}{2}} N_{2}^{-\frac{\alpha-1}{2}} N_{3}^{-\nu-\frac{\alpha-1}{2}} \\
& \leq N_{3}^{\frac{3}{2}-\alpha-\nu} N_{1}^{-\frac{\alpha}{2}}+N_{1}^{-(\alpha-1)} N_{2}^{-\frac{\alpha-1}{2}} N_{3}^{-\nu-\frac{\alpha-1}{2}} \\
& \lesssim N_{1}^{-\nu-\frac{3(\alpha-1)}{2}}+N_{1}^{-(\alpha-1+\nu)\left(1-\frac{\alpha}{2}\right)-(\alpha-1)},
\end{aligned}
$$

where to the last step we used $N_{3}^{\frac{3}{2}-\alpha-\nu}<N_{1}^{\frac{3}{2}-\alpha-\nu}$. When $\alpha>\alpha_{0}$, we have $(\alpha-1+$ $\nu)\left(1-\frac{\alpha}{2}\right)+(\alpha-1)>s+\delta_{0}$ and $\frac{3(\alpha-1)}{2}+\nu>s+\delta_{0}$. This completes the proof of Lemma 11.5

To deal with other situations, we need to execute other Algorithms.

-Subcase B-5(a): Exact one of $a_{2}, a_{3}$ is of type $(\mathbf{G})$ or $(\mathbf{C})$ and the other is of type (D)

Without loss of generality, we may assume that $a_{2}$ is of type $(\mathrm{G})$ or $(\mathrm{C})$ and $a_{3}$ is of type (D), since in the regime $N_{1} \gg N_{2}, N_{3}$, the second and third positions in $\mathcal{N}_{3}(\cdot, \cdot, \cdot)$ are similar. By Corollary 11.5, it suffices to consider the case $L_{1} \ll N_{2} \wedge N_{3} \leq N_{2}$. By implementing Algorithm 2, it suffices to estimate the quantity

$$
\sum_{k, k^{\prime}: k \neq k^{\prime}} \mathbb{E}^{\mathcal{C}}\left[\left|\sigma_{k k^{\prime}}^{(3)}\right|^{2}\right]+\sup _{k} \mathbb{E}^{\mathcal{C}}\left[\left|\sigma_{k k}^{(3)}\right|^{2}\right],
$$


and then take the square root of the obtained upper bound, where $\mathcal{C}$ is the $\sigma$-algebra generated by $\mathcal{B}_{L_{1} \vee L_{2}}$,

$$
\sigma_{k k^{\prime}}^{(3)}=\sum_{\substack{k_{1}, k_{1}^{\prime}, k_{2}, k_{2}^{\prime}, k_{3} \\ k_{1}=k+k_{2}-k_{3} \\ k_{1}^{\prime}=k^{\prime}+k_{2}^{\prime}-k_{3}}} \bar{a}_{1}\left(k_{1}^{\prime}\right) a_{1}\left(k_{1}\right) a_{2}\left(k_{2}^{\prime}\right) \bar{a}_{2}\left(k_{2}\right) S_{k_{1}^{\prime}, k_{2}^{\prime}, k_{3}} S_{k_{1}, k_{2}, k_{3}} \mathbf{1}_{|k|,\left|k^{\prime}\right|>N_{1}}
$$

and

$$
a_{j}\left(k_{j}\right)=\sum_{\left|k_{j}^{*}\right| \sim N_{j}} \mathbf{1}_{\left|k_{j}-k_{j}^{*}\right| \leq L_{j}} h_{k_{j} k_{j}^{*}}^{(q)} \frac{g_{k_{j}^{*}}}{\left[k_{j}^{*}\right]^{\frac{\alpha}{2}}}, \quad j=1,2 .
$$

Lemma 11.6. Assume that $a_{1}, a_{2}$ are both of type $(G)$ or $(C)$ and $L_{1} \ll N_{2}$. Then by implementing Algorithm 2, outside a set of probability $<\mathrm{e}^{-N_{(1)}^{\theta} R}$ and modulo small powers of $N_{(1)}$ :

- If $N_{2} \vee N_{3}<N_{1}^{1-\frac{\alpha}{2}}$, then

$$
\left|\widetilde{\mathcal{U}}_{L_{1}, L_{2}, L_{3}}^{N_{1}, N_{2}, N_{3}}\right| \lesssim N_{1}^{-\frac{\alpha}{2}} N_{2}^{-\frac{\alpha}{2}} L_{1}^{-\nu} L_{2}^{\frac{1}{2}-\nu}\left(N_{2} \vee N_{3}\right)\left(N_{2} \wedge N_{3}\right)^{\frac{1}{4}}\left\|a_{3}\right\|_{l^{2}}
$$

- If $N_{2} \vee N_{3} \geq N_{1}^{1-\frac{\alpha}{2}}$, then

$$
\begin{aligned}
\left|\widetilde{\mathcal{U}}_{L_{1}, L_{2}, L_{3}}^{N_{1}, N_{2}, N_{3}}\right| & \lesssim N_{1}^{-\frac{\alpha}{2}} N_{2}^{-\frac{\alpha}{2}} L_{1}^{-\nu} L_{2}^{\frac{1}{2}-\nu}\left[N_{2}^{\frac{1}{2}} N_{3}^{\frac{1}{4}}+N_{3}^{\frac{1}{4}} N_{1}^{\frac{1}{2}\left(1-\frac{\alpha}{2}\right)} \min \left\{N_{2}^{\frac{1}{2}}, N_{1}^{\frac{1}{2}\left(1-\frac{\alpha}{2}\right)}\right\}\right]\left\|a_{3}\right\|_{l^{2}} \\
& +N_{1}^{-\frac{\alpha}{2}} N_{2}^{-\frac{\alpha}{2}} L_{1}^{-\nu} L_{2}^{\frac{1}{2}-\nu} \cdot N_{2}^{\frac{1}{2}} N_{1}^{\frac{1}{2}\left(1-\frac{\alpha}{2}\right)} \min \left\{N_{3}^{\frac{1}{4}}, N_{1}^{\frac{1}{4}\left(1-\frac{\alpha}{2}\right)}\right\}\left\|a_{3}\right\|_{l^{2}} .
\end{aligned}
$$

If $a_{1}, a_{3}$ are both of type $(G)$ or $(C)$ and $a_{2}$ is of type $(D)$ and $L_{1} \ll N_{3}$, the above estimates hold by switching $N_{2}$ to $N_{3}, a_{3}$ to $a_{2}$ and $L_{2}$ to $L_{3}$.

Proof. From our assumption, $a_{1}\left(k_{1}^{\prime}\right), a_{1}\left(k_{1}\right)$ are independent of $a_{2}\left(k_{2}\right), a_{2}\left(k_{2}^{\prime}\right)$. Noticing that

$$
\begin{aligned}
\left|\sigma_{k k^{\prime}}^{(3)}\right|^{2}= & \sum_{\begin{array}{c}
k_{2}, k_{2}^{\prime}, k_{3} \\
m_{2}, m_{2}^{\prime}, m_{3}
\end{array}} S_{k^{\prime}+k_{2}^{\prime}-k_{3}, k_{2}^{\prime}, k_{3}} S_{k+k_{2}-k_{3}, k_{2}, k_{3}} S_{k^{\prime}+m_{2}^{\prime}-m_{3}, m_{2}^{\prime}, m_{3}} S_{k+m_{2}-m_{3}, m_{2}, m_{3}} \\
& \times \bar{a}_{1}\left(k^{\prime}+k_{2}^{\prime}-k_{3}\right) a_{1}\left(k+k_{2}-k_{3}\right) a_{1}\left(k^{\prime}+m_{2}^{\prime}-m_{3}\right) \bar{a}_{1}\left(k+m_{2}-m_{3}\right) \\
& \times a_{2}\left(k_{2}^{\prime}\right) \bar{a}_{2}\left(k_{2}\right) \bar{a}_{2}\left(m_{2}^{\prime}\right) a_{2}\left(m_{2}\right) .
\end{aligned}
$$

By using the independence and Cauchy-Schwartz, we have

$$
\begin{aligned}
& \sum_{k, k^{\prime}:|k|>N_{1},\left|k^{\prime}\right|>N_{1}} \mathbb{E}\left[\left|\sigma_{k k^{\prime}}^{(3)}\right|^{2}\right] \\
& \lesssim\left(N_{1} N_{2}\right)^{-2 \alpha} \sum_{\substack{k, k^{\prime}, k_{2}, k_{2}^{\prime}, k_{3} \\
m_{2}, m_{2}^{\prime}, m_{3}}} S_{k^{\prime}+k_{2}^{\prime}-k_{3}, k_{2}^{\prime}, k_{3}} S_{k+k_{2}-k_{3}, k_{2}, k_{3}} S_{k^{\prime}+m_{2}^{\prime}-m_{3}, m_{2}^{\prime}, m_{3}} S_{k+m_{2}-m_{3}, m_{2}, m_{3}} \mathbf{1}_{|k|,\left|k^{\prime}\right|>N_{1}} \\
& \times \prod_{j=1}^{2}\left\|h_{k_{j} k_{j}^{*}}^{(q)}\right\|_{l_{k_{j}}^{\infty} l_{k_{j}^{*}}^{2}}^{4}\left(\mathbf{1}_{\left|k_{2}-k_{2}^{\prime}\right| \leq L_{2},\left|m_{2}-m_{2}^{\prime}\right| \leq L_{2}}+\mathbf{1}_{\left|k_{2}-m_{2}\right|<L_{2},\left|k_{2}^{\prime}-m_{2}^{\prime}\right|<L_{2}}\right)
\end{aligned}
$$

Denote by $\mathcal{C}_{1}$ the contribution from the indicator $\mathbf{1}_{\left|k_{2}-k_{2}^{\prime}\right| \leq L_{2},\left|m_{2}-m_{2}^{\prime}\right| \leq L_{2}}$. We first sum over $m_{3}$, using the fact that

$$
\sum_{m_{3}} S_{k+m_{2}-m_{3}, m_{2}, m_{3}} S_{k^{\prime}+m_{2}^{\prime}-m_{3}, m_{2}^{\prime}, m_{3}} \lesssim 1
$$



and then we sum over $k, k^{\prime}$ by using the inequality (11.4). This yields

$$
\begin{aligned}
\mathcal{C}_{1} & \lesssim\left(N_{1} N_{2}\right)^{-2 \alpha} \prod_{j=1}^{2}\left\|h_{k_{j} k_{j}^{*}}^{(q)}\right\|_{l_{k_{j}}^{\infty} l_{k_{j}^{*}}^{2}}^{4} \sum_{k_{2}, k_{2}^{\prime}, k_{3}, m_{2}, m_{2}^{\prime}} \mathbf{1}_{\substack{\left|k_{2}-k_{2}^{\prime}\right|<m_{2}-m_{2}^{\prime}\left|<L_{2}\\
\right| N_{2}}} B\left(k_{2}, k_{3}\right) B\left(k_{2}^{\prime}, k_{3}\right) \\
& \lesssim\left(N_{1} N_{2}\right)^{-2 \alpha} L_{2} \prod_{j=1}^{2}\left\|h_{k_{j} k_{j}^{*}}^{(q)}\right\|_{l_{k_{j}}^{\infty} l_{k_{j}^{*}}^{2}}^{4} \sum_{k_{2}, k_{2}^{\prime}, k_{3}, m_{2}} \mathbf{1}_{\left|k_{2}-k_{2}^{\prime}\right|<L_{2}} B\left(k_{2}, k_{3}\right) B\left(k_{2}^{\prime}, k_{3}\right),
\end{aligned}
$$

where

$$
B\left(k_{2}, k_{3}\right):=\min \left\{1+\frac{N_{1}^{2-\alpha}}{\left\langle k_{2}-k_{3}\right\rangle},\left\langle k_{2}-k_{3}\right\rangle\right\} .
$$

Now by Schur's test and Cauchy-Schwartz, we have

$$
\begin{aligned}
& \sum_{k_{3}, m_{2}} \sum_{k_{2}, k_{2}^{\prime}} \mathbf{1}_{\left|k_{2}-k_{2}^{\prime}\right|<L_{2}} B\left(k_{2}, k_{3}\right) B\left(k_{2}^{\prime}, k_{3}\right) \\
& \leq L_{2} N_{2}\left\|B\left(k_{2}, k_{3}\right)\right\|_{l_{k_{2}, k_{3}}^{2}}^{2} \lesssim\left\{\begin{array}{l}
L_{2}\left(N_{2} \vee N_{3}\right)^{4}\left(N_{2} \wedge N_{3}\right), \text { if } N_{2} \vee N_{3}<N_{1}^{1-\frac{\alpha}{2}} \\
L_{2} N_{2}^{2}\left(N_{3}+N_{1}^{2-\alpha} \min \left\{N_{3}, N_{1}^{1-\frac{\alpha}{2}}\right\}\right), \text { if } N_{2} \vee N_{3} \geq N_{1}^{1-\frac{\alpha}{2}} .
\end{array}\right.
\end{aligned}
$$

Therefore,

$$
\mathcal{C}_{1} \lesssim\left(N_{1} N_{2}\right)^{-2 \alpha} L_{1}^{-4 \nu} L_{2}^{2-4 \nu} \cdot\left\{\begin{array}{l}
\left(N_{2} \vee N_{3}\right)^{4}\left(N_{2} \wedge N_{3}\right), \text { if } N_{2} \vee N_{3}<N_{1}^{1-\frac{\alpha}{2}} ; \\
N_{2}^{2} N_{3}+N_{1}^{2-\alpha} N_{2}^{2} \min \left\{N_{3}, N_{1}^{1-\frac{\alpha}{2}}\right\}, \text { if } N_{2} \vee N_{3} \geq N_{1}^{1-\frac{\alpha}{2}} .
\end{array}\right.
$$

Denote by $\mathcal{C}_{2}$ the contribution from the indicator $\mathbf{1}_{\left|k_{2}-m_{2}\right| \leq L_{2},\left|k_{2}^{\prime}-m_{2}^{\prime}\right| \leq L_{2}}$. Similarly, we first sum over $m_{3}$ and then $k, k^{\prime}$ using (11.4), we have

$$
\begin{aligned}
\mathcal{C}_{2} & \lesssim\left(N_{1} N_{2}\right)^{-2 \alpha} \prod_{j=1}^{2} \|\left. h_{k_{j} k_{j}^{*}}^{(q)}\right|_{l_{k_{j}}^{\infty}} ^{4} \sum_{k_{j}^{*}}^{2} \sum_{k_{2}, k_{2}^{\prime}, k_{3}, m_{2}, m_{2}^{\prime}} \mathbf{1}_{\left|k_{2}-m_{2}\right|<L_{2}-m_{2}^{\prime} \mid<L_{2}} B\left(k_{2}, k_{3}\right) B\left(k_{2}^{\prime}, k_{3}\right) \\
& \lesssim\left(N_{1} N_{2}\right)^{-2 \alpha} L_{2}^{2} \prod_{j=1}^{2}\left\|h_{k_{j} k_{j}^{*}}^{(q)}\right\|_{l_{k_{j}} l_{k_{j}^{*}}^{*}}^{4} \sum_{k_{2}, k_{2}^{\prime}, k_{3}} B\left(k_{2}, k_{3}\right) B\left(k_{2}^{\prime}, k_{3}\right) .
\end{aligned}
$$

For fixed $k_{3}$, we have

$$
\sum_{k_{2}} B\left(k_{2}, k_{3}\right) \lesssim\left\{\begin{array}{l}
\left(N_{2} \vee N_{3}\right) N_{2}, \text { if } N_{2} \vee N_{3}<N_{1}^{1-\frac{\alpha}{2}} \\
N_{2}+\min \left\{N_{1}^{1-\frac{\alpha}{2}} N_{2}, N_{1}^{2-\alpha}\right\}, \text { if } N_{2} \vee N_{3} \geq N_{1}^{1-\frac{\alpha}{2}}
\end{array}\right.
$$

Therefore, modulo small powers,

$$
\mathcal{C}_{2} \lesssim\left(N_{1} N_{2}\right)^{-2 \alpha} L_{1}^{-4 \nu} L_{2}^{2-4 \nu} \cdot\left\{\begin{array}{l}
\left(N_{2} \vee N_{3}\right)^{4}\left(N_{2} \wedge N_{3}\right), \text { if } N_{2} \vee N_{3}<N_{1}^{1-\frac{\alpha}{2}} \\
N_{2}^{2} N_{3}+N_{3} N_{1}^{2-\alpha} \min \left\{N_{2}^{2}, N_{1}^{2-\alpha}\right\}, \text { if } N_{2} \vee N_{3} \geq N_{1}^{1-\frac{\alpha}{2}} .
\end{array}\right.
$$

Implementing Algorithm 2, the proof of Lemma 11.6 is complete.

Corollary 11.7. Assume that $N_{1} \gg N_{2}, N_{3}$ and $a_{1}, a_{2}$ are of type $(G)$ or $(C), a_{3}$ is of type (D). Assume that $L_{1} \ll N_{2}$, then outside a set of probability $<\mathrm{e}^{-N_{(1)}^{\theta} R}$ and modulo a small power $N_{1}^{\epsilon}, \epsilon<\kappa^{-0.1}$, we have

$$
\left|\widetilde{\mathcal{U}}_{L_{1}, L_{2}, L_{3}}^{N_{1}, N_{2}, N_{3}}\right| \lesssim N_{1}^{-s-2 \delta_{0}} .
$$

The same estimate holds if we switch $a_{2}$ and $a_{3}$. 
Proof. The proof is just a numerical computation. Assume that $a_{2}$ is of type (G) or (C). First we assume that $N_{2} \vee N_{3}<N_{1}^{1-\frac{\alpha}{2}}$. Then by (i) of Lemma 11.6, if $N_{3} \leq N_{2}<N_{1}^{1-\frac{\alpha}{2}}$, $\left|\widetilde{\mathcal{U}}_{L_{1}, L_{2}, L_{3}}^{N_{1}, N_{2}, N_{3}}\right| \lesssim\left(N_{1} N_{2}\right)^{-\frac{\alpha}{2}} N_{3}^{-s} L_{2}^{\frac{1}{2}-\nu} \cdot N_{2} N_{3}^{\frac{1}{4}}<N_{1}^{-\frac{\alpha}{2}} L_{2}^{\frac{1}{2}-\nu} N_{2}^{\frac{5}{4}-s-\frac{\alpha}{2}}<L_{2}^{\frac{1}{2}-\nu} N_{1}^{-\frac{\alpha}{2}+\left(\frac{5}{4}-s-\frac{\alpha}{2}\right)\left(1-\frac{\alpha}{2}\right)}$

$$
\lesssim N_{1}^{-s} \cdot L_{2}^{\frac{1}{2}-\nu} N_{1}^{-\frac{(1-s) \alpha}{2}+\left(\frac{5}{4}-\frac{\alpha}{2}\right)\left(1-\frac{\alpha}{2}\right)} .
$$

Note that if $N_{2} \leq N_{3}<N_{1}^{1-\frac{\alpha}{2}}$, we have the same upper bound. When $L_{2}^{\frac{1}{2}-\nu}<$ $N_{1}^{\frac{(1-s) \alpha}{2}-\left(\frac{5}{4}-\frac{\alpha}{2}\right)\left(1-\frac{\alpha}{2}\right)-2 \delta_{0}}$, this bound is conclusive. When $L_{2}^{\frac{1}{2}-\nu} \geq N_{1}^{\frac{(1-s) \alpha}{2}-\left(\frac{5}{4}-\frac{\alpha}{2}\right)\left(1-\frac{\alpha}{2}\right)-2 \delta_{0}}$, we alternatively apply (1) of Lemma 11.4 to get

$\left|\tilde{\mathcal{U}}_{L_{1}, L_{2}, L_{3}}^{N_{1}, N_{2}, N_{3}}\right| \lesssim N_{1}^{-\frac{\alpha}{2}} N_{2}^{\frac{1}{2}-(\alpha-1)} L_{2}^{-\nu} N_{3}^{\frac{1}{2}-s} \leq N_{1}^{-\frac{\alpha}{2}} L_{2}^{-\nu}\left(N_{2} \vee N_{3}\right)^{2-\alpha-s}$

$$
<L_{2}^{-\nu} N_{1}^{-\frac{\alpha}{2}+(2-\alpha-s)\left(1-\frac{\alpha}{2}\right)} \leq N_{1}^{-\frac{\alpha}{2}+(2-\alpha-s)\left(1-\frac{\alpha}{2}\right)} \cdot N_{1}^{-\frac{2 \nu}{1-2 \nu} \cdot\left[\frac{(1-s) \alpha}{2}-\left(\frac{5}{4}-\frac{\alpha}{2}\right)\left(1-\frac{\alpha}{2}\right)-2 \delta_{0}\right]} .
$$

By numerical computation, when $\alpha>1.069\left(<\alpha_{0}\right)$, we are able to choose sufficiently small $\sigma>0$, such that $\left|\widetilde{\mathcal{U}}_{L_{1}, L_{2}, L_{3}}^{N_{1}, N_{2}, N_{3}}\right| \lesssim N_{1}^{-s-2 \delta_{0}}$, which is conclusive.

Next we assume that $N_{2} \vee N_{3} \geq N_{1}^{1-\frac{\alpha}{2}}$. First we deal with the case $N_{2}, N_{3} \geq N_{1}^{1-\frac{\alpha}{2}}$. By (ii) of Lemma 11.6, we have

$$
\begin{aligned}
\left|\widetilde{\mathcal{U}}_{L_{1}, L_{2}, L_{3}}^{N_{1}, N_{2}, N_{3}}\right| & \lesssim \underbrace{N_{1}^{-\frac{\alpha}{2}} N_{2}^{-\frac{\alpha}{2}} L_{2}^{\frac{1}{2}-\nu} N_{3}^{\frac{1}{4}-s} N_{2}^{\frac{1}{2}}}_{\text {I }}+\underbrace{N_{1}^{-\frac{\alpha}{2}} N_{2}^{-\frac{\alpha}{2}} L_{2}^{\frac{1}{2}-\nu} N_{3}^{\frac{1}{4}-s} N_{1}^{1-\frac{\alpha}{2}}}_{\text {II }} \\
& +\underbrace{N_{1}^{-\frac{\alpha}{2}} N_{2}^{-\frac{\alpha}{2}} L_{2}^{\frac{1}{2}-\nu} N_{2}^{\frac{1}{2}} N_{1}^{\frac{3}{4}\left(1-\frac{\alpha}{2}\right)} N_{3}^{-s}}_{\text {III }} .
\end{aligned}
$$

Using $L_{2} \ll N_{2}$, we have

$$
\mathrm{I} \leq N_{1}^{-\frac{\alpha}{2}} N_{2}^{1-\frac{\alpha}{2}-\nu} N_{3}^{\frac{1}{4}-s} \leq N_{1}^{-\frac{\alpha}{2}+1-\frac{\alpha}{2}-\nu+\frac{1}{4}-s}<N_{1}^{-s-2 \delta_{0}},
$$

for sufficiently small $\sigma>0$, since $\alpha+\min \left\{\frac{1}{2}-\frac{\alpha}{4}, \frac{7(\alpha-1)}{4}\right\}>\frac{5}{4}$. Next,

$$
\begin{gathered}
\mathrm{II} \leq N_{1}^{-(\alpha-1)} N_{2}^{-\frac{\alpha}{2}} L_{2}^{\frac{1}{2}-\nu} N_{1}^{\frac{1}{4}-\left(\frac{1}{2}-\frac{\alpha}{4}\right)}=N_{1}^{-\frac{3(\alpha-1)}{4}} N_{2}^{-\frac{\alpha}{2}} L_{2}^{\frac{1}{2}-\nu}<N_{1}^{-\frac{\alpha}{2}\left(1-\frac{\alpha}{2}\right)-\frac{3(\alpha-1)}{4}} L_{2}^{\frac{1}{2}-\nu} . \\
\text { III } \leq N_{1}^{-\frac{\alpha}{2}+\frac{3}{4}\left(1-\frac{\alpha}{2}\right)} L_{2}^{\frac{1}{2}-\nu} N_{2}^{-\frac{\alpha-1}{2}} N_{3}^{-s} \leq N_{1}^{-(\alpha-1)-\frac{1+\alpha}{4}\left(1-\frac{\alpha}{2}\right)} L_{2}^{\frac{1}{2}-\nu} .
\end{gathered}
$$

Since $N_{1}^{-(\alpha-1)-\frac{1+\alpha}{4}\left(1-\frac{\alpha}{2}\right)}<N_{1}^{-\frac{\alpha}{2}\left(1-\frac{\alpha}{2}\right)-\frac{3(\alpha-1)}{4}}$, we have

$$
\mathrm{I}+\mathrm{II}+\mathrm{III} \lesssim N_{1}^{-s-2 \delta_{0}}+L_{2}^{\frac{1}{2}-\nu} N_{1}^{-s} N_{1}^{s-\frac{\alpha}{2}\left(1-\frac{\alpha}{2}\right)-\frac{3(\alpha-1)}{4}} .
$$

When $L_{2}^{\frac{1}{2}-\nu}<N_{1}^{\frac{\alpha}{2}\left(1-\frac{\alpha}{2}\right)+\frac{3(\alpha-1)}{4}-s-2 \delta_{0}}$, the upper bound 11.12 is conclusive. When $L_{2}^{\frac{1}{2}-\nu} \geq N_{1}^{\frac{\alpha}{2}\left(1-\frac{\alpha}{2}\right)+\frac{3(\alpha-1)}{4}-s-2 \delta_{0}}$, we alternatively apply (2) of Lemma 11.4 to obtain

$$
\begin{aligned}
& \left|\widetilde{\mathcal{U}}_{L_{1}, L_{2}, L_{3}}^{N_{1}, N_{2}, N_{3}}\right| \lesssim\left(N_{2}^{\frac{1}{2}}+N_{1}^{1-\frac{\alpha}{2}}\right) N_{1}^{-\frac{\alpha}{2}} N_{2}^{-(\alpha-1)} L_{2}^{-\nu} N_{3}^{-s} \\
& \lesssim N_{1}^{-\frac{\alpha}{2}} N_{2}^{\frac{3}{2}-\alpha} N_{3}^{-s} L_{2}^{-\nu}+N_{1}^{-(\alpha-1)} N_{2}^{-(\alpha-1)} N_{3}^{-s} L_{2}^{-\nu}
\end{aligned}
$$

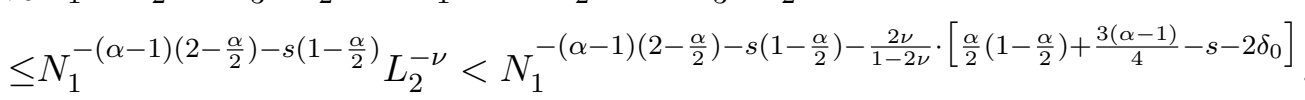

By numerical computation, we are able to choose sufficiently small $\sigma>0$, such that the above upper bound can be bounded by $N_{1}^{-s-2 \delta_{0}}$, provided that $\alpha>1.0698\left(<\alpha_{0}\right)$. Thus

$$
\mathrm{I}+\mathrm{II}+\mathrm{III} \lesssim N_{1}^{-s-2 \delta_{0}} .
$$


Finally we assume that $N_{2} \vee N_{3} \geq N_{1}^{1-\frac{\alpha}{2}}>N_{2} \wedge N_{3}$. By using (2) of Lemma 11.6, we have

$$
\left|\widetilde{\mathcal{U}}_{L_{1}, L_{2}, L_{3}}^{N_{1}, N_{2}, N_{3}}\right| \lesssim N_{1}^{-\frac{\alpha}{2}} N_{2}^{-\frac{\alpha}{2}} L_{2}^{\frac{1}{2}-\nu} N_{3}^{\frac{1}{4}-s} \cdot N_{2}^{\frac{1}{2}} N_{1}^{\frac{1}{2}\left(1-\frac{\alpha}{2}\right)}, \text { if } N_{3}<N_{1}^{1-\frac{\alpha}{2}} \leq N_{2},
$$

and

$$
\left|\widetilde{\mathcal{U}}_{L_{1}, L_{2}, L_{3}}^{N_{1}, N_{2}, N_{3}}\right| \lesssim N_{1}^{-\frac{\alpha}{2}} N_{2}^{-\frac{\alpha-1}{2}} L_{2}^{\frac{1}{2}-\nu} N_{3}^{-s} \cdot N_{3}^{\frac{1}{4}} N_{1}^{\frac{1}{2}\left(1-\frac{\alpha}{2}\right)}, \text { if } N_{2}<N_{1}^{1-\frac{\alpha}{2}} \leq N_{3} .
$$

If $N_{2} \wedge N_{3}<N_{1}^{1-\frac{\alpha}{2}} \leq N_{2} \vee N_{3}$, we have

$$
\left|\widetilde{\mathcal{U}}_{L_{1}, L_{2}, L_{3}}^{N_{1}, N_{2}, N_{3}}\right| \lesssim N_{1}^{-\frac{\alpha}{2}+\frac{1}{2}\left(1-\frac{\alpha}{2}\right)} N_{1}^{\frac{1}{4}-s} L_{2}^{\frac{1}{2}-\nu} \leq N_{1}^{-s} \cdot N_{1}^{-\frac{3(\alpha-1)}{4}} L_{2}^{\frac{1}{2}-\nu}
$$

When $L_{2}^{\frac{1}{2}-\nu}<N_{1}^{\frac{3(\alpha-1)}{4}-2 \delta_{0}}$, this bound is conclusive. When $L_{2}^{\frac{1}{2}-\nu} \geq N_{1}^{\frac{3(\alpha-1)}{4}-2 \delta_{0}}$, we can alternatively apply (2) of Lemma 11.4 to obtain

$$
\begin{aligned}
\left|\widetilde{\mathcal{U}}_{L_{1}, L_{2}, L_{3}}^{N_{1}, N_{2}, N_{3}}\right| & \lesssim\left(N_{2} \wedge N_{3}\right)^{\frac{1}{2}} N_{1}^{\frac{1}{2}-\frac{\alpha}{4}} \cdot N_{1}^{-\frac{\alpha}{2}} N_{2}^{-(\alpha-1)} L_{2}^{-\nu} N_{3}^{-s} \\
& \leq N_{1}^{\frac{1}{2}-\frac{3 \alpha}{4}}\left(N_{2} \vee N_{3}\right)^{-(\alpha-1)}\left(N_{2} \wedge N_{3}\right)^{\frac{1}{2}-s} L_{2}^{-\nu},
\end{aligned}
$$

since $\alpha<\frac{6}{5}$ and $\alpha-1<s$. Thus

$$
\left|\widetilde{\mathcal{U}}_{L_{1}, L_{2}, L_{3}}^{N_{1}, N_{2}, N_{3}}\right| \lesssim N_{1}^{\frac{1}{2}-\frac{3 \alpha}{4}+\left(\frac{1}{2}-s-(\alpha-1)\right)\left(1-\frac{\alpha}{2}\right)-\frac{2 \nu}{1-2 \nu} \cdot\left[\frac{3(\alpha-1)}{4}-2 \delta_{0}\right]} .
$$

By numerical computation, when $\alpha>1.0724\left(<\alpha_{0}\right)$, we are able to choose sufficiently small $\sigma>0$, such that

$$
\left|\widetilde{\mathcal{U}}_{L_{1}, L_{2}, L_{3}}^{N_{1}, N_{2}, N_{3}}\right| \lesssim N_{1}^{-s-2 \delta_{0}}
$$

This completes the proof of Corollary 11.7

-Subcase B-5(b): $a_{2}, a_{3}$ are both of type (G) or (C) and $L_{1} \ll N_{2} \wedge N_{3}$

First we assume that $N_{3} \gg N_{2}$ and $L_{3} \gtrsim N_{2}$ (similar for the case $N_{2} \gg N_{3}$ and $\left.L_{2} \gtrsim N_{3}\right)$. In this case, we cannot execute Algorithm 1 since $h_{k_{2} k_{2}^{*}}^{(q)}$ are not independent of $g_{k_{3}^{*}}$. Instead, we apply Lemma 11.4 . When $N_{3}<N_{1}^{1-\frac{\alpha}{2}}$, we have

$$
\begin{aligned}
\left|\widetilde{\mathcal{U}}_{L_{1}, L_{2}, L_{3}}^{N_{1}, N_{2}, N_{3}}\right| & \lesssim\left(N_{2} N_{3}\right)^{\frac{1}{2}} N_{1}^{-\frac{\alpha}{2}} N_{2}^{-\frac{\alpha-1}{2}} N_{3}^{-(\alpha-1)} L_{3}^{-\nu} \lesssim N_{1}^{-\frac{\alpha}{2}} N_{2}^{1-\frac{\alpha}{2}-\nu} N_{3}^{\frac{3}{2}-\alpha} \\
& \leq N_{3}^{\frac{5}{2}-\frac{3 \alpha}{2}-\nu} N_{1}^{-\frac{\alpha}{2}}<N_{1}^{-\frac{\alpha}{2}+\left(\frac{5-3 \alpha}{2}-\nu\right)\left(1-\frac{\alpha}{2}\right)} .
\end{aligned}
$$

When $N_{3} \gg N_{2} \geq N_{1}^{1-\frac{\alpha}{2}}$, we have

$$
\begin{aligned}
\left|\widetilde{\mathcal{U}}_{L_{1}, L_{2}, L_{3}}^{N_{1}, N_{2}, N_{3}}\right| & \lesssim\left(N_{2}^{\frac{1}{2}}+N_{1}^{1-\frac{\alpha}{2}}\right) \cdot N_{1}^{-\frac{\alpha}{2}} N_{2}^{-\frac{\alpha-1}{2}} N_{3}^{-(\alpha-1)} L_{3}^{-\nu} \\
& \leq N_{1}^{-\left(2-\frac{\alpha}{2}\right)(\alpha-1)-\nu}+N_{1}^{-\frac{\alpha}{2}+\left(\frac{5-3 \alpha}{2}-\nu\right)\left(1-\frac{\alpha}{2}\right)}
\end{aligned}
$$

When $N_{3} \geq N_{1}^{1-\frac{\alpha}{2}}>N_{2}$, we have

$$
\begin{aligned}
\left|\widetilde{\mathcal{U}}_{L_{1}, L_{2}, L_{3}}^{N_{1}, N_{2}, N_{3}}\right| & \lesssim N_{1}^{\frac{1}{2}\left(1-\frac{\alpha}{2}\right)} N_{2}^{\frac{1}{2}} \cdot N_{1}^{-\frac{\alpha}{2}} N_{2}^{-\frac{\alpha-1}{2}} N_{3}^{-(\alpha-1)} L_{3}^{-\nu} \\
& \lesssim N_{1}^{-\frac{\alpha-1}{2}-\frac{\alpha}{4}} N_{2}^{1-\frac{\alpha}{2}-\nu} N_{3}^{-(\alpha-1)} \leq N_{1}^{-\frac{\alpha}{2}+\left(\frac{5-3 \alpha}{2}-\nu\right)\left(1-\frac{\alpha}{2}\right)} .
\end{aligned}
$$

Therefore,

$$
\left|\widetilde{\mathcal{U}}_{L_{1}, L_{2}, L_{3}}^{N_{1}, N_{2}, N_{3}}\right| \lesssim N_{1}^{-\frac{\alpha}{2}+\left(\frac{5-3 \alpha}{2}-\nu\right)\left(1-\frac{\alpha}{2}\right)}+N_{1}^{-\left(2-\frac{\alpha}{2}\right)(\alpha-1)-\nu} .
$$

By numerical computation, when $\alpha>1.0918\left(<\alpha_{0}\right)$, we are able to choose sufficiently small $\sigma>0$, small enough, such that the right side of 11.15 is bounded by $N_{1}^{-s-2 \delta_{0}}$. 
Now we assume that $a_{2}, a_{3}$ are both of type (G) or (C) and $L_{1} \ll N_{2} \wedge N_{3}, L_{2} \vee L_{3} \ll$ $N_{2} \wedge N_{3}$. In this case, we execute Algorithm 1 and the goal is to estimate $\mathbb{E}^{\mathcal{C}}\left[\left|\widetilde{\mathcal{U}}_{L_{1}, L_{2}, L_{3}}^{N_{1}, N_{2}, N_{3}}\right|^{2}\right]=\sum_{|k|>N_{1}} \sum_{\substack{\left(k_{1}, k_{2}, k_{3}\right) \in \Gamma(k) \\\left(m_{1}, m_{2}, m_{3}\right) \in \Gamma(k)}} \mathbb{E}^{\mathcal{C}}\left[a_{1}\left(k_{1}\right) \bar{a}_{1}\left(m_{1}\right)\right] \cdot \mathbb{E}^{\mathcal{C}}\left[\bar{a}_{2}\left(k_{2}\right) a_{2}\left(m_{2}\right) a_{3}\left(k_{3}\right) \bar{a}_{3}\left(m_{3}\right)\right]$,

where $\mathcal{C}=\mathcal{B}_{<\max \left\{L_{1}, L_{2}, L_{3}\right\}}$. Here we used the fact that $a_{1}\left(k_{1}\right), a_{1}\left(m_{1}\right)$ are independent of $a_{j}\left(k_{j}\right), a_{j}\left(m_{j}\right)$ for $j=2,3$ since $N_{1} \gg N_{2}, N_{3}$ and $L_{1} \ll N_{2} \wedge N_{3}$. Using the independence and Cauchy-Schwartz, we have

$$
\begin{aligned}
& \mathbb{E}^{\mathcal{C}}\left[\left|\widetilde{\mathcal{U}}_{L_{1}, L_{2}, L_{3}}^{N_{1}, N_{2}, N_{3}}\right|^{2}\right] \lesssim\left(N_{1} N_{2} N_{3}\right)^{-\alpha} \prod_{j=1}^{3}\left\|h_{k_{j} k_{j}^{*}}^{(q)}\right\|_{l_{k_{j}}^{\infty} l_{k_{j}^{*}}^{2}}^{2} \\
& \times \sum_{\substack{k, k_{2}, k_{3}, m_{2}, m_{3} \\
|k|>N_{1}}} S_{k+k_{2}-k_{3}, k_{2}, k_{3}} S_{k+m_{2}-m_{3}, m_{2}, m_{3}}\left(\begin{array}{c}
\mathbf{1}_{\left|k_{2}-m_{2}\right|<L_{2}}+\mathbf{1}_{\left|k_{2}-k_{3}\right|<L_{2} \vee L_{3}} \\
\left|k_{3}-m_{3}\right|<L_{3} \\
\left|m_{2}-m_{3}\right|<L_{2} \vee L_{3}
\end{array}\right) .
\end{aligned}
$$

To sum the second line of the right side, we first sum over $m_{3}$ by using $\sum_{m_{3}} S_{k+m_{2}-m_{3}, m_{2}, m_{3}} \lesssim$ 1 and then sum over $|k|>N_{1}$ by using (11.4). This procedure yields

$$
\mathbb{E}^{\mathcal{C}}\left[\left|\widetilde{\mathcal{U}}_{L_{1}, L_{2}, L_{3}}^{N_{1}, N_{2}, N_{3}}\right|^{2}\right] \lesssim\left(N_{1} N_{2} N_{3}\right)^{-\alpha} \prod_{j=1}^{3}\left\|h_{k_{j} k_{j}^{*}}^{(q)}\right\|_{l_{k_{j}}^{\infty} l_{k_{j}^{*}}^{2}}^{2} \sum_{m_{2}, k_{2}, k_{3}}\left(\mathbf{1}_{\left|k_{2}-m_{2}\right|<L_{2}}+\mathbf{1}_{\left|k_{2}-k_{3}\right|<L_{2} \vee L_{3}}\right) B\left(k_{2}, k_{3}\right),
$$

where

$$
B\left(k_{2}, k_{3}\right)=\min \left\{\left\langle k_{2}-k_{3}\right\rangle, 1+\frac{N_{1}^{2-\alpha}}{\left\langle k_{2}-k_{3}\right\rangle}\right\} .
$$

To estimate the contribution from $\mathbf{1}_{\left|k_{2}-m_{2}\right|<L_{2}}$, we recall that

$$
\sum_{k_{2}, k_{3}} B\left(k_{2}, k_{3}\right) \lesssim\left\{\begin{array}{l}
\left(N_{2} \vee N_{3}\right)^{2}\left(N_{2} \wedge N_{3}\right), \text { if } N_{2} \vee N_{3}<N_{1}^{1-\frac{\alpha}{2}} \\
N_{2} N_{3}+\left(N_{2} \vee N_{3}\right) \min \left\{N_{1}^{2-\alpha},\left(N_{2} \wedge N_{3}\right) N_{1}^{1-\frac{\alpha}{2}}\right\}, \text { if } N_{2} \vee N_{3} \geq N_{1}^{1-\frac{\alpha}{2}} .
\end{array}\right.
$$

To estimate the contribution from $\mathbf{1}_{\left|k_{2}-k_{3}\right|<L_{2} \vee L_{3}}$, we note that if $L_{2} \vee L_{3} \leq N_{1}^{1-\frac{\alpha}{2}}$,

$$
\sum_{k_{2}, k_{3}} \mathbf{1}_{\left|k_{2}-k_{3}\right|<L_{2} \vee L_{3}} B\left(k_{2}, k_{3}\right) \lesssim\left(L_{2} \vee L_{3}\right)^{2}\left(N_{2} \wedge N_{3}\right),
$$

and if $L_{2} \vee L_{3}>N_{1}^{1-\frac{\alpha}{2}}$,

$$
\begin{aligned}
& \sum_{k_{2}, k_{3}} \mathbf{1}_{\left|k_{2}-k_{3}\right|<L_{2} \vee L_{3}} B\left(k_{2}, k_{3}\right)=\sum_{k_{2}, k_{3}} \mathbf{1}_{\left|k_{2}-k_{3}\right| \leq N_{1}^{1-\frac{\alpha}{2}}} B\left(k_{2}, k_{3}\right)+\sum_{k_{2}, k_{3}} \mathbf{1}_{N_{1}^{1-\frac{\alpha}{2}}<\left|k_{2}-k_{3}\right|<L_{2} \vee L_{3}} B\left(k_{2}, k_{3}\right) \\
& \lesssim N_{1}^{1-\frac{\alpha}{2}} \min \left\{\left(N_{2} \wedge N_{3}\right) N_{1}^{1-\frac{\alpha}{2}}, N_{2} N_{3}\right\}+\left(L_{2} \vee L_{3}\right)\left(N_{2} \wedge N_{3}\right)+\left(N_{2} \wedge N_{3}\right) N_{1}^{2-\alpha} \text {. }
\end{aligned}
$$

In what follows, we may assume that $N_{2} \leq N_{3}$. Therefore, modulo possible small powers of $N_{1}$, we obtain that

$$
\mathbb{E}^{\mathcal{C}}\left[\left|\widetilde{\mathcal{U}}_{L_{1}, L_{2}, L_{3}}^{N_{1}, N_{2}, N_{3}}\right|^{2}\right] \lesssim \mathrm{I}+\mathrm{II},
$$

where

$$
\mathrm{I}=\left(N_{1} N_{2} N_{3}\right)^{-\alpha}\left(L_{1} L_{2} L_{3}\right)^{-2 \nu}\left(L_{2} \vee L_{3}\right)\left\{\begin{array}{l}
\left(N_{2} \vee N_{3}\right)^{2}\left(N_{2} \wedge N_{3}\right), \text { if } N_{3}<N_{1}^{1-\frac{\alpha}{2}} \\
N_{2} N_{3}+N_{3} \min \left\{N_{1}^{2-\alpha}, N_{2} N_{1}^{1-\frac{\alpha}{2}}\right\}, \text { if } N_{3} \geq N_{1}^{1-\frac{\alpha}{2}},
\end{array}\right.
$$

and

$$
\mathrm{II}=\left(N_{1} N_{2} N_{3}\right)^{-\alpha}\left(L_{1} L_{2} L_{3}\right)^{-2 \nu}\left(L_{2} \vee L_{3}\right)^{2} N_{2} N_{3},
$$

if $L_{2} \vee L_{3} \leq N_{1}^{1-\frac{\alpha}{2}}$, while

II $=\left(N_{1} N_{2} N_{3}\right)^{-\alpha}\left(L_{1} L_{2} L_{3}\right)^{-2 \nu} N_{2}\left[N_{1}^{1-\frac{\alpha}{2}} \min \left\{N_{2} N_{1}^{1-\frac{\alpha}{2}}, N_{2} N_{3}\right\}+\left(L_{2} \vee L_{3}\right) N_{2}+N_{2} N_{1}^{2-\alpha}\right]$,

if $L_{2} \vee L_{3}>N_{1}^{1-\frac{\alpha}{2}}$. 
Let us first estimate I: If $N_{3}<N_{1}^{1-\frac{\alpha}{2}}$, using our hypothesis $L_{2} \vee L_{3} \ll N_{2}$, we can bound I by

$$
\begin{aligned}
\mathrm{I} & \lesssim N_{1}^{-\alpha} L_{2}^{-2 \nu} L_{3}^{-2 \nu}\left(L_{2} \vee L_{3}\right) N_{3}^{2-\alpha} N_{2}^{1-\alpha} \lesssim N_{1}^{-\alpha} N_{2}^{2-\alpha-2 \nu} N_{3}^{2-\alpha} \leq N_{1}^{-\alpha} N_{3}^{2(2-\alpha-\nu)} \\
& \leq N_{1}^{-\alpha+2(2-\alpha-\nu)\left(1-\frac{\alpha}{2}\right)} .
\end{aligned}
$$

In the case $N_{3} \geq N_{1}^{1-\frac{\alpha}{2}}$, if $N_{3} \geq N_{2} \geq N_{1}^{1-\frac{\alpha}{2}}$, using $L_{2} \vee L_{3} \ll N_{2}$, we have

$$
\begin{aligned}
\mathrm{I} & \leq N_{1}^{-\alpha} N_{2}^{-(\alpha-1)} N_{3}^{-(\alpha-1)} N_{2}^{1-2 \nu}+N_{1}^{-2(\alpha-1)} N_{2}^{-\alpha} N_{3}^{-(\alpha-1)} N_{2}^{1-2 \nu} \\
& \leq N_{1}^{-\alpha} N_{2}^{2-\alpha-2 \nu} N_{3}^{-(\alpha-1)}+N_{1}^{-2(\alpha-1)} N_{2}^{-(\alpha+2 \nu-1)} N_{3}^{-(\alpha-1)} \\
& \leq N_{1}^{-\alpha} N_{3}^{(3-2 \nu-2 \alpha)}+N_{1}^{-2(\alpha-1)} N_{1}^{-(2(\alpha-1)+2 \nu)\left(1-\frac{\alpha}{2}\right)} .
\end{aligned}
$$

If $N_{3} \geq N_{1}^{1-\frac{\alpha}{2}}>N_{2}$, we have

$$
\begin{aligned}
\mathrm{I} & \leq N_{1}^{-\alpha+1-\frac{\alpha}{2}}\left(N_{2} N_{3}\right)^{-(\alpha-1)}\left(L_{2} \vee L_{3}\right)^{1-2 \nu} \leq N_{1}^{1-\frac{3 \alpha}{2}} N_{2}^{2-\alpha-2 \nu} N_{3}^{-(\alpha-1)} \\
& \leq N_{1}^{1-\frac{3 \alpha}{2}} N_{1}^{(2-\alpha-2 \nu)\left(1-\frac{\alpha}{2}\right)} N_{1}^{-(\alpha-1)\left(1-\frac{\alpha}{2}\right)}
\end{aligned}
$$

Therefore, we obtain that

$$
\mathrm{I} \lesssim\left\{\begin{array}{l}
N_{1}^{-3(\alpha-1)-2 \nu}+N_{1}^{-\alpha+2(2-\alpha-\nu)\left(1-\frac{\alpha}{2}\right)}, \text { if } N_{3} \geq N_{2} \geq N_{1}^{1-\frac{\alpha}{2}} \\
N_{1}^{-\alpha+2(2-\alpha-\nu)\left(1-\frac{\alpha}{2}\right)}, \text { if } N_{3} \geq N_{1}^{1-\frac{\alpha}{2}}>N_{2},
\end{array}\right.
$$

and in particular,

$$
\mathrm{I} \lesssim N_{1}^{-3(\alpha-1)-2 \nu}+N_{1}^{-\alpha+2(2-\alpha-\nu)\left(1-\frac{\alpha}{2}\right)} .
$$

When $\alpha>1.1205\left(<\alpha_{0}\right)$, we are able to choose sufficiently small $\sigma>0$, such that the right side of 11.16$)<N_{1}^{-2 s-4 \delta_{0}}$.

It remains to estimate II. First we assume that $L_{2} \vee L_{3} \leq N_{1}^{1-\frac{\alpha}{2}}$, since $N_{2} \geq L_{2} \vee L_{3}$, we have

$$
\begin{aligned}
\mathrm{II} & =N_{1}^{-\alpha}\left(N_{2} N_{3}\right)^{-(\alpha-1)}\left(L_{1} L_{2} L_{3}\right)^{-2 \nu}\left(L_{2} \vee L_{3}\right)^{2} \\
& \leq N_{1}^{-\alpha}\left(L_{2} \vee L_{3}\right)^{2(1-\nu)-(\alpha-1)} \leq N_{1}^{-\alpha+(2(1-\nu)-(\alpha-1))\left(1-\frac{\alpha}{2}\right)} .
\end{aligned}
$$

By numerical computation, when $\alpha>\frac{10}{9}\left(<\alpha_{0}\right)$, we are able to choose sufficiently small $\sigma>0$, such that

$$
N_{1}^{-\alpha+2\left(1-\nu-\frac{\alpha-1}{2}\right)\left(1-\frac{\alpha}{2}\right)}<N_{1}^{-2 s-4 \delta_{0}}
$$

which is conclusive. Finally we assume that $L_{2} \vee L_{3}>N_{1}^{1-\frac{\alpha}{2}}$, then

$$
\begin{aligned}
\mathrm{II} & \leq\left(N_{1} N_{2} N_{3}\right)^{-\alpha}\left(L_{2} \vee L_{3}\right)^{-2 \nu}\left(N_{2} N_{3} N_{1}^{2-\alpha}+\left(L_{2} \vee L_{3}\right) N_{2} N_{3}\right) \\
& \leq N_{1}^{-2(\alpha-1)}\left(L_{2} \vee L_{3}\right)^{-2 \nu-(\alpha-1)}+N_{1}^{-\alpha}\left(L_{2} \vee L_{3}\right)^{1-2 \nu-(\alpha-1)} \\
& \leq N_{1}^{-2(\alpha-1)-(2 \nu+(\alpha-1))\left(1-\frac{\alpha}{2}\right)}+N_{1}^{-\alpha+1-2 \nu-(\alpha-1)} \\
& \leq N_{1}^{-2(\alpha-1)-2\left(\nu+\frac{\alpha-1}{2}\right)\left(1-\frac{\alpha}{2}\right)}+N_{1}^{-2(\alpha-1+\nu)} .
\end{aligned}
$$

By numerical computation, when $\alpha>\frac{10}{9}\left(<\alpha_{0}\right)$, we are able to choose sufficiently small $\sigma>0$, such that the right hand side of the above inequality is smaller than $N_{1}^{-2 s-4 \delta_{0}}$.

Implementing Algorithm 1. The proof of tri-linear estimate for Case B-5 is complete. In summary, the proof of Proposition 3.7 is completely finished. 


\section{Appendix 1: Proof of Proposition 1.2}

By the triangle inequality and the Wiener chaos estimate, the second assertion in Proposition 1.2 follows from the expectation bound of $\left|z_{2 k+1}^{\omega}(t, x)\right|$, hence we will only show that

$$
\mathbb{E}\left[\left|z_{2 k+1}^{\omega}(t, x)\right|^{2}\right] \leq C_{0} t^{2 j}(2 j+1) !\left(\frac{(2 j-1) ! !}{j !}\right)^{2} .
$$

Without loss of generality, we take $t>0$. Recall the expression 1.5 and the equation of $z_{2 j+1}$, we have the recurrence relation for the coefficient $c_{j}\left(t, k_{1}, \cdots, k_{2 j+1}\right)$ :

$$
\begin{aligned}
c_{j}\left(t, k_{1}, \cdots, k_{2 j+1}\right)= & -\sum_{\substack{j_{1}, j_{2}, j_{3} \\
j_{1}+j_{2}+j_{3}=j-1}} \int_{0}^{t} \mathrm{e}^{i\left(t-t^{\prime}\right)\left|k_{1}-k_{2}+\cdots-k_{2 j}+k_{2 j+1}\right|^{\alpha}} d t^{\prime} \\
& \times c_{j_{1}}\left(t^{\prime}, k_{1}, \cdots, k_{2 j_{1}+1}\right) c_{j_{2}}\left(t^{\prime}, k_{2 j_{1}+2}, \cdots, k_{2 j_{1}+2 j_{2}+2}\right) c_{j_{3}}\left(t^{\prime}, k_{2 j_{2}+3}+\cdots+k_{2 j+1}\right) .
\end{aligned}
$$

Note that $c_{0}\left(t, k_{1}\right)=1$ and $\left|c_{1}\left(t, k_{1}\right)\right| \leq t$. Define recurrently the series $\left\{\kappa_{j}\right\}_{j \geq 0}$ by $\kappa_{0}=1$ and

$$
\kappa_{j}:=\frac{1}{j} \sum_{\substack{j_{1}, j_{2}, j_{3} \\ j_{1}+j_{2}+j_{3}=j-1}} \kappa_{j_{1}} \kappa_{j_{2}} \kappa_{j_{3}} .
$$

By induction we deduce that for any $j \geq 0$,

$$
\left|c_{j}\left(t, n_{1}, \cdots, n_{2 j+1}\right)\right| \leq \kappa_{j} t^{j} .
$$

Next we determine $\kappa_{j}$. Considering the power series

$$
f(x)=\sum_{j=0}^{\infty} \kappa_{j} z^{j},
$$

the recurrence relation 11.18 implies that $f^{\prime}(z)=f(z)^{3}, f(0)=0$. Solving this ODE we obtain that $f(z)=(1-2 z)^{-\frac{1}{2}}$, hence

$$
\kappa_{j}=\frac{(2 j-1) ! !}{j !} .
$$

Therefore, we obtain that

$$
\left|c_{j}\left(t, k_{1}, \cdots, k_{2 j+1}\right)\right| \leq \kappa_{j} t^{j}:=\frac{(2 j-1) ! !}{j !} t^{j} .
$$

Thus

$$
\begin{aligned}
& \mathbb{E}\left[\left|z_{2 j+1}^{\omega}(t, x)\right|^{2}\right]=\sum_{\substack{k_{1}, \cdots, k_{2 j+1} \\
m_{1}, \cdots, m_{2 j+1}}} c_{j}\left(t, k_{1}, \cdots, k_{2 j+1}\right) \bar{c}_{j}\left(t, m_{1}, \cdots, m_{2 j+1}\right) \\
& \times \mathrm{e}_{k_{1}-k_{2}+\cdots+k_{2 j+1}} \mathrm{e}_{-m_{1}+m_{2}-\cdots-m_{2 j+1}} \mathbb{E}\left[\prod_{j^{\prime}=1}^{2 j+1} \frac{g_{k_{j^{\prime}} \iota^{\prime}}^{\iota_{j^{\prime}}} g_{m_{j^{\prime}}}^{-\iota_{j^{\prime}}}}{\left[k_{j^{\prime}}\right]^{\frac{\alpha}{2}}\left[m_{j^{\prime}}\right]^{\frac{\alpha}{2}}}\right],
\end{aligned}
$$

where $\iota_{j^{\prime}} \in\{+1,-1\}$ and we use the convention $g_{k}^{+1}=g_{j}$ and $g_{k}^{-1}=\bar{g}_{k}$. Using the independence of Gaussians and (11.19), we have

$$
\mathbb{E}\left[\left|z_{2 j+1}^{\omega}(t, x)\right|^{2}\right] \leq \sum_{k_{1}, \cdots, k_{2 j+1}} \kappa_{j}^{2} t^{2 j} \cdot(2 j+1) ! \prod_{j^{\prime}=1}^{2 j+1} \frac{1}{\left[k_{j^{\prime}}\right]^{\frac{\alpha}{2}}} \leq C_{0} t^{2 j}(2 j+1) !\left(\frac{(2 j-1) ! !}{j !}\right)^{2},
$$

where the extra factor $(2 j+1)$ ! comes from the number of pairings for the index

$$
\left(k_{1}, \cdots, k_{2 j+1} ; m_{1}, \cdots, m_{2 j+1}\right) .
$$

This completes the proof of (11.17). 


\section{Appendix 2: Proof of Lemma 2.3}

It suffices to prove the first inequality

$$
\left\|\chi_{T}(t) u\right\|_{X_{p, q}^{0, \gamma}} \lesssim T^{\gamma_{1}-\gamma}\|u\|_{X_{p, q}^{0, \gamma_{1}}}
$$

with $u \in X_{q, p}^{0, \gamma}$ satisfying $\left.u\right|_{t=0}=0$, where $1 \leq p \leq \infty, 0<\gamma<\gamma_{1}<1+\frac{1}{q^{\prime}}$ and $1 \leq q<\infty$, since we may regard $\left\|\widetilde{\Theta}_{k k^{\prime}}\left(\lambda, \lambda^{\prime}\right)\right\|_{L_{\lambda^{\prime}}^{2} l_{k^{\prime}}^{2}}$ as $\widetilde{u}(\lambda, k)$. We decompose $u=u_{1}+u_{2}$, where

$$
\mathcal{F}_{t, x} u_{1}(\tau, k)=\mathbf{1}_{\left.|\tau-| k\right|^{\alpha} \mid \geq \frac{1}{T}} \mathcal{F}_{t, x} u(\tau, k), \quad \mathcal{F}_{t, x} u_{2}(\tau, k)=\mathbf{1}_{\left.|\tau-| k\right|^{\alpha} \mid<\frac{1}{T}} \mathcal{F}_{t, x} u(\tau, k) .
$$

To prove 11.20 , it suffices to show that, uniformly in $k \in \mathbb{Z}$,

$$
\begin{aligned}
& \text { (a) }\left\|\left\langle\tau-|k|^{\alpha}\right\rangle^{\gamma}\left(\widehat{\chi_{T}} * \mathcal{F}_{t, x} u_{1}\right)(\tau, k)\right\|_{L_{\tau}^{q}} \lesssim T^{\gamma_{1}-\gamma}\left\|\left\langle\eta-|k|^{\alpha}\right\rangle^{\gamma_{1}} \mathcal{F}_{t, x} u(\eta, k)\right\|_{L_{\eta}^{q}} ; \\
& \text { (b) }\left\|\left\langle\tau-|k|^{\alpha}\right\rangle^{\gamma}\left(\widehat{\chi_{T}} * \mathcal{F}_{t, x} u_{2}\right)(\tau, k)\right\|_{L_{\tau}^{q}} \lesssim T^{\gamma_{1}-\gamma}\left\|\left\langle\eta-|k|^{\alpha}\right\rangle^{\gamma_{1}} \mathcal{F}_{t, x} u(\eta, k)\right\|_{L_{\eta}^{q}} .
\end{aligned}
$$

To prove (a), we note that

$$
\left\langle\tau-|k|^{\alpha}\right\rangle^{\gamma}\left(\widehat{\chi}_{T} * \mathcal{F}_{t, x} u_{1}\right)(\tau, k)=\int_{\mathbb{R}} K_{T}^{+}\left(\tau-|k|^{\alpha}, \omega\right)\langle\omega\rangle^{\gamma_{1}} \widetilde{u}(\omega, k) d \omega,
$$

where

$$
K_{T}^{+}(\lambda, \omega)=\widehat{\chi}(T(\lambda-\omega)) \frac{T\langle\lambda\rangle^{\gamma}}{\left\langle\omega \gamma^{\gamma}\right.} \mathbf{1}_{|\omega| \geq \frac{1}{T}} .
$$

By Schur's test, it suffices to show that

$$
\sup _{\lambda \in \mathbb{R}} \int_{\mathbb{R}}\left|K_{T}^{+}(\lambda, \omega)\right| d \omega \leq C_{1} T^{\gamma_{1}-\gamma}, \quad \sup _{\omega \in \mathbb{R}} \int_{\mathbb{R}}\left|K_{T}^{+}(\lambda, \omega)\right| d \lambda \leq C_{2} T^{\gamma_{1}-\gamma}
$$

with $C_{1}, C_{2}$ independent of $T$. One can check these two inequalities by direct computation, here we explain it in an informal way. Since $\widehat{\chi}$ is a Schwartz function, $|\lambda-\omega|$ is essentially bounded by $O\left(\frac{1}{T}\right)$. Due to the fact that $|\omega| \geq \frac{1}{T}, \lambda$ is essentially constraint in the region $|\lambda| \lesssim \frac{1}{T}$. Since the length of the integration is of size $\frac{1}{T}$, we deduce that two integrations are bounded by $O(1) T^{\gamma_{1}-\gamma}$.

The proof of (b) exploits the cancellation from the condition $\left.u\right|_{t=0}=0$. By the Fourier inversion formula, we have

$$
\int_{\mathbb{R}} \widetilde{u}(\omega, k) d \omega=0
$$

Therefore, with $\lambda=\tau-|k|^{\alpha}$,

$$
\begin{aligned}
\left(\widehat{\chi}_{T} * \mathcal{F}_{t, x} u_{2}\right)(\lambda, k) & =\int_{|\omega|<\frac{1}{T}} T \widehat{\chi}(T(\lambda-\omega)) \widetilde{u}(\omega, k) d \omega \\
& =\int_{|\omega|<\frac{1}{T}} T \widetilde{u}(\omega, k)[\widehat{\chi}(T(\lambda-\omega))-\widehat{\chi}(T \lambda)] d \omega+T \widehat{\chi}(T \lambda) \int_{|\omega| \geq \frac{1}{T}} \widetilde{u}(\omega, k) d \omega
\end{aligned}
$$

By Hölder's inequality,

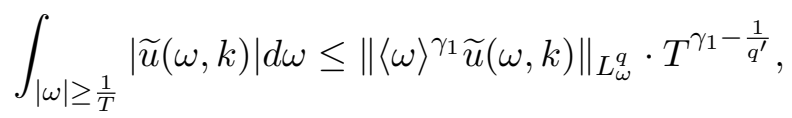

we have

$$
\left\|\langle\lambda\rangle^{\gamma} T \widehat{\chi}(T \lambda) \int_{|\omega| \geq \frac{1}{T}} \widetilde{u}(\omega, k) d \omega\right\|_{L_{\lambda}^{q}} \lesssim T^{\gamma_{1}-\gamma}\left\|\langle\omega\rangle^{\gamma_{1}} \widetilde{u}(\omega, k)\right\|_{L_{\omega}^{q}} .
$$

Finally, from the fact that $[\widehat{\chi}(T(\lambda-\omega))-\widehat{\chi}(T \lambda)] \mathbf{1}_{T|\omega|<1}=O\left(|T \omega|\langle T \lambda\rangle^{-100}\right)$, we deduce by Hölder that

$\left.\mid \int_{|\omega|<\frac{1}{T}} T \widetilde{u}(\omega, k) \widehat{\chi}(T(\lambda-\omega))-\widehat{\chi}(T \lambda)\right] d \omega \mid \leq T^{2} O\left(\langle T \lambda\rangle^{-100}\right)\left\|\langle\omega\rangle^{\gamma_{1}} \widetilde{u}(\omega, k)\right\|_{L_{\omega}^{q}}\left\|\frac{|\omega|}{\langle\omega\rangle^{\gamma_{1}}}\right\|_{L^{q^{\prime}}\left(|\omega| \leq \frac{1}{T}\right)}$.

Multiplying the right hand side by $\langle\lambda\rangle^{\gamma}$ and then taking the $L^{q}$ norm in $\lambda$, we obtain the desired upper bound $T^{\gamma_{1}-\gamma}$. This proves (b). The proof of Lemma 2.3 is now complete. 


\section{REFERENCES}

[1] A. Bényi, T. Oh, O. Pocovnicu, Higher order expansions for the probabilistic local Cauchy theory of the cubic nonlinear Schrödinger equation on $\mathbb{R}^{3}$, Trans. Amer. Math. Soc., Ser. B 6 (2019), 114-160.

[2] J. Bourgain, Fourier transform restriction phenomena for certain lattice subsets and applications to nonlinear evolution equations. I. Schrödinger equations, Geom. Funct. Anal., 3 (1993) 107-156.

[3] J. Bourgain, Periodic nonlinear Schrödinger equation and invariant measures, Comm. Math. Phys., 166 (1994) 1-26.

[4] J. Bourgain, Invariant measures for the 2d-defocusing nonlinear Schrödinger equation, Comm. Math. Phys., 176 (1996) 421-445.

[5] J. Bourgain, A. Bulut, Invariant Gibbs measure evolution for the radial nonlinear wave equation on the 3d ball, J. Funct. Anal., 266 (2014) 2319-2340.

[6] J. Bourgain, A. Bulut, Almost sure global well posedness for the radial nonlinear Schrödinger equation on the unit ball I: the 2D case, Ann. Inst. H. Poincaré Anal. Non Linéaire, 31 (2014) 1267-1288.

[7] J. Bourgain, A. Bulut, Almost sure global well posedness for the radial nonlinear Schrödinger equation on the unit ball II: the 3D case, J. Eur. Math. Soc., 16 (2014) 1289-1325.

[8] B. Bringmann, Almost sure local well-posedness for a derivative nonlinear wave equation, to appear in Int. Math. Res. Noti., arXiv:1809.00220.

[9] B. Bringmann, Invariant Gibbs measures for the three-dimensional wave equation with a Hartree nonlinearity II: Dynamics, arXiv:2009.04616.

[10] N. Burq, L. Thomann, N. Tzvetkov, Remarks on the Gibbs measures for nonlinear dispersive equations, Ann. Fac. Sci. Toulouse Math., (6) 27 (2018) 527-597.

[11] N. Burq, N. Tzvetkov, Random data Cauchy theory for supercritical wave equations I. Local theory, Invent. Math., 173 (2008) 449-475.

[12] N. Burq, N. Tzvetkov, Probabilistic well-posedness for the cubic wave equation, J. Eur. Math. Soc., 16 (2014) 1-30

[13] Y. Cho, G. Hwant, S. Kwon, S. Lee, Well-posedness and ill-posedness for the cubic fractional Schrödinger equations, Discrete and Continuous Dynamical Systems, 35 (2015) 2863-2880.

[14] G. Da Prato, A. Debussche, Strong solutions to the stochastic quantization equations, Ann. Probab., 31 (2003) 1900-1916.

[15] S. Demirbas, Almost sure global well-posedness for the fractional cubic Schrödinger equation on the torus, Canad. Math. Bull., 58 (2015) 471-485.

[16] Y. Deng, A-R. Nahmod, H. Yue, Optimal local well-posedness for the periodic derivative nonlinear Schrödinger equation, arXiv:1905.04352.

[17] Y. Deng, A-R. Nahmod, H. Yue, Invariant Gibbs measures and global strong solutions for nonlinear Schrödinger equations in dimension two, arXiv: 1910.98492.

[18] Y. Deng, A-R. Nahmod, H. Yue, Random tensors, propagation of randomness, and nonlinear dispersive equations, arXiv:2006.09285.

[19] A. Ionescu, F. Pusateri, Nolinear fractional Schrödinger equations in one dimension, J. Func. Anal., 266 (2014) 139-176.

[20] M. Gubinelli, P. Imkeller, P. Perkowski, Paracontrolled distributions and singular PDEs, Forum Math. 3 (2015) e6, $75 \mathrm{pp}$

[21] M. Gubinelli, H. Koch, T. Oh, Paracontrolled approach to the three-dimensional stochastic nonlinear wave equation with quadratic nonlinearity, arXiv:1811.07808 [math.AP].

[22] M. Hairer, A theory of regularity structures, Invent. Math., 198 (2014) 269-504.

[23] K. Kirkpatrick, E. Lenzmann, G. Staffilani, On the continuum limit for discrete NLS with long-range lattice interactions, Comm. Math. Phys., 317 (2013) 563-591.

[24] N. Laskin, Fractional quantum mechanics and Lévy path integrals, Phys. Lett., 268 (2000) 298-305.

[25] T. Oh, M. Okamoto, L. Tolomeo, Focusing $\Phi_{4}^{3}$-model with a Hartree-type nonlinearity, arXiv:2009.03251.

[26] T. Oh, L. Thomann, A pedestrian approach to the invariant Gibbs measures for the 2-d defocusing nonlinear Schrdinger equations, Stoch. PDE. Anal. Comp., 6, (2018), 397445.

[27] T. Oh, N. Tzvetkov, Y. Wang, Solving the 4NLS with white noise initial data, arXiv:1902.06169.

[28] C.-M. Sun, N. Tzvetkov, Gibbs measure dynamics for the fractional NLS, SIAM J. Math. Anal., $52(5),(2020)$ 4638-4704.

[29] J. Thirouin, On the growth of Sobolev norms of solutions of the fractional defocusing NLS equation on the circle, Annales de l'Institut Henri Poincaré (C) Analyse non linéaire, (2017) 509-531.

Université de Cergy-Pontoise, Cergy-Pontoise, F-95000,UMR 8088 du CNRS

E-mail address: nikolay.tzvetkov@u-cergy.fr

E-mail address: chenmin.sun@u-cergy.fr 